\title{
Temporal patterns in the pathophysiology of dialysis patients : an epidemiological approach
}

Citation for published version (APA):

Usvyat, L. A. (2013). Temporal patterns in the pathophysiology of dialysis patients : an epidemiological approach. [Doctoral Thesis, Maastricht University]. Universitaire Pers Maastricht. https://doi.org/10.26481/dis.20130607lu

Document status and date:

Published: 01/01/2013

DOI:

10.26481/dis.20130607lu

Document Version:

Publisher's PDF, also known as Version of record

\section{Please check the document version of this publication:}

- A submitted manuscript is the version of the article upon submission and before peer-review. There can be important differences between the submitted version and the official published version of record.

People interested in the research are advised to contact the author for the final version of the publication, or visit the DOI to the publisher's website.

- The final author version and the galley proof are versions of the publication after peer review.

- The final published version features the final layout of the paper including the volume, issue and page numbers.

Link to publication

\footnotetext{
General rights rights.

- You may freely distribute the URL identifying the publication in the public portal. please follow below link for the End User Agreement:

www.umlib.nl/taverne-license

Take down policy

If you believe that this document breaches copyright please contact us at:

repository@maastrichtuniversity.nl

providing details and we will investigate your claim.
}

Copyright and moral rights for the publications made accessible in the public portal are retained by the authors and/or other copyright owners and it is a condition of accessing publications that users recognise and abide by the legal requirements associated with these

- Users may download and print one copy of any publication from the public portal for the purpose of private study or research.

- You may not further distribute the material or use it for any profit-making activity or commercial gain

If the publication is distributed under the terms of Article $25 \mathrm{fa}$ of the Dutch Copyright Act, indicated by the "Taverne" license above, 
Temporal patterns in the pathophysiology of dialysis patients: an epidemiological approach 
C Len Usvyat, Maastricht 2013

ISBN : 9789461592354

Cover-drawing: Daniel Albert Usvyat - 15 years old Gabrielle Sophie Usvyat - 8 years old Lay out: $\quad$ Tiny Wouters

Production: Datawyse I Universitaire Pers Maastricht 


\title{
Temporal patterns in the pathophysiology of dialysis patients: an epidemiological approach
}

\author{
Proefschrift
}

ter verkrijging van de graad van doctor aan de Universiteit Maastricht, op gezag van de Rector Magnificus, Prof. dr. L.L.G. Soete, volgens het besluit van het College van Decanen, in het openbaar te verdedigen op vrijdag 7 juni 2013 om 10.00 uur

door

Len Usvyat

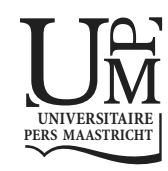




\section{Promotores:}

Prof. dr. J.P. Kooman

Prof. dr. P. Kotanko (Renal Research Institute, New York)

\section{Copromotores}

Prof. dr. N.W. Levin (Renal Research Institute, New York)

Dr. F.M. van der Sande

\section{Beoordelingscommissie:}

Prof. dr. M.P. van Dieijen-Visser (voorzitter)

Prof. dr. A.M.W.J. Schols

Prof. dr. C.D.A. Stehouwer

Prof. dr. P.M. van Wee (VUMC) 


\section{CONTENTS}

$\begin{array}{lll}\text { Chapter } 1 & \text { General introduction } & 7\end{array}$

Part A Oscillatory trends in dialysis patients and their outcomes $\quad 19$

Chapter 2 Circadian variations in body temperature during dialys 21

Chapter 3 Seasonal variations in mortality, clinical, and laboratory 37 parameters in hemodialysis patients: a 5-year cohort study

Part B Longitudinal, non-oscillatory, trends in dialysis patients 53

Chapter $4 \quad$ Blood pressure stability in hemodialysis patients confers $\quad 55$ a survival advantage: results from a large retrospective cohort study

Chapter 5 Relation between trends in body temperature and outcome in incident hemodialysis patients

Chapter 6 Dynamics of hospitalizations in dialysis patients: results from the RRI database

Chapter 7 The Monitoring Dialysis Outcomes (MONDO) initiative

Chapter 8 Dynamics of inter-dialytic weight gain, systolic blood pressure, serum albumin, and C-reactive protein levels in chronic dialysis patients before death

Chapter 9 General discussion / summary

Dankwoord 



\section{Chapter 1}

General introduction 
Chapter 1 


\section{Introduction}

Hemodialysis (HD) is the most commonly used treatment modality for patients suffering from end-stage renal disease (ESRD). Worldwide, around 2 million people are being treated with renal replacement therapy $(\mathrm{RRT})^{1}$ of which nearly $90 \%$ are treated with HD. HD is a life-saving therapy; however, despite its success, mortality and morbidity in dialysis patients still remains very high. According to United States Renal Dialysis System (USRDS) data, only $50 \%$ of incident dialysis patients are still alive after 3 years of dialysis therapy ${ }^{2}$.

\section{Short background of hemodialysis}

The fact that dialysis patients often suffer from severe comorbidities and have already experienced a high disease burden at the start of dialysis ${ }^{3}$, and the limitations of standard HD treatment likely contribute to these adverse outcomes. These limitations deserve some explanation. HD is usually performed 3 times weekly for approximately 4 hours each. During an HD session, uremic toxins and the excess water and sodium, which accumulate during the inter-dialytic period, are removed and electrolyte and acid base disturbances are corrected. HD combines diffusion (solute transport by concentration differences, by which uremic toxins are removed and acid-base disturbances are corrected) and convection (primarily removal of fluid).

The discontinuous nature of HD treatment is in sharp contrast to the function of the healthy kidneys, which serve to continuously maintain a stable internal environment in response to multiple internal and external stressors. Moreover, due to the fact that treatment is only performed during relatively short periods of time, the clearance of (small) uremic toxins, the best example of which is urea, during standard HD is equal to a glomerular filtration rate of around $15 \mathrm{ml} / \mathrm{min}^{4}$. The removal of protein bound uremic toxins and those with molecular weights over about 20 Daltons is much lower.

\section{Complications in dialysis patients: "patient- and treatment-related"}

Next to the increased mortality and high hospitalization rate, the disease burden in dialysis patients is characterized by high complication rates. These complications may be divided into those related to the HD treatment itself ("procedure-related") and those related to the patient ("patient-related") ${ }^{5}$. Of course, there is no sharp division between each type of complication; for example intra-dialytic hypotension may contribute to chronic cardiovascular damage, whereas chronic cardiac dysfunction may put the patient at risk for intra-dialytic hypotension ${ }^{6,7}$. 
The treatment-related complication addressed in this thesis is intra-dialytic hypotension, which not only leads to discomfort for the patient, but may also be a risk factor for cardiac, cerebral ${ }^{6,7}$, and intestinal ischemia. The immediate cause of intradialytic hypotension is the reduction of blood volume due to the removal of fluid during a relatively short period of time without adequate refilling of fluid from the interstitial space. The maintenance of blood pressure during fluid removal is also dependent on adequate cardiovascular compensatory mechanisms, such as an increase in myocardial contractility as well as arteriolar and venous constriction. However, the normal vasoconstrictor response during HD may be compromised by thermal changes evoked by the treatment itself. Body temperature (BT) increases during dialysis, which leads to reflex vasodilatation in the skin, counteracting the normal hemodynamic response to a decline in blood volume. The causes of the increase in BT during dialysis have not been completely elucidated. It has been suggested that initial vasoconstriction in response to hypovolemia may result in "skin isolation", followed by an increase in BT and reflex vasodilatation followed by hypotension ${ }^{8}$. However, later studies have shown that this mechanism could not be the sole explanation, given the fact that BT also increases in dialysis sessions without ultrafiltration ${ }^{9}$. More research into the pathogenesis of the BT increase during HD might therefore be of relevance for both pathophysiological as well as therapeutic reasons.

Important "patient-related" complications in dialysis patients addressed in this thesis are cardiovascular disease and malnutrition. Cardiovascular disease in dialysis patients has a different profile compared to the general population, as evidenced by a high prevalence of systolic and diastolic dysfunction, as well as increased vascular stiffness and calcifications ${ }^{10,11}$. The pathogenesis of cardiovascular complications is multifactorial, with hypertension, chronic fluid overload and abnormalities in calciumphosphate balance as important etiologies ${ }^{11-13}$. Fluid overload is very common in dialysis patients; it is caused by an inadequate intra-dialytic removal of both intra- and inter-dialytic gain of excess sodium and fluid in these, mostly anuric, patients.

In HD patients, fluid overload can lead to acute complications, notably in the form of acute pulmonary edema, but also to chronic cardiovascular abnormalities in the form of hypertension, left ventricular hypertrophy and dilatation, left ventricular systolic and diastolic dysfunction and subsequent congestive heart failure ${ }^{14}$. Recent studies have clearly related fluid overload to mortality ${ }^{15}$.

Malnutrition in dialysis patients is characterized by reduced dietary intake, a reduction in muscle mass and is associated with a decrease in serum albumin levels ${ }^{16}$. Concomitant inflammation has been implicated as an important factor in both the pathogenesis of cardiovascular disease, particularly of blood vessels, as well as in 
malnutrition. This is evidenced by the strong prognostic power of C-reactive protein (CRP) levels and the widespread prevalence of the malnutrition-inflammationatherosclerosis (MIA) syndrome in dialysis patients ${ }^{12,17}$, in which these three comorbid factors are clustered.

\section{"Reverse epidemiology" paradox}

Various clinical and biochemical parameters related to cardiovascular disease, inflammation and malnutrition have been established as major risk factors for morbidity and mortality in dialysis patients. However, some of these risk factors show a different prognostic trend as compared to the general population. Whereas low serum albumin and high CRP levels are consistently related to mortality in dialysis patients, other risk factors show a different trend. For instance, patients with high blood pressure levels and high body mass index (BMI) appear to have better survival in dialysis patients as compared to patients with low blood pressure and $\mathrm{BMI}^{18,19}$. Moreover, not only high ${ }^{20}$, but also low inter-dialytic weight gain is related to mortality. These observations led to the term "reverse epidemiology paradox" in dialysis patients ${ }^{21}$. These less common outcomes may be explained by the possibility that comorbid factors such as cardiac failure or malnutrition, which are directly related to mortality, may also have a strong negative effect on risk factors such as blood pressure and BMI. Also, part of this paradox might be due to the fact that in most epidemiological studies in dialysis patients, the relation between risk factors and mortality is assessed at a single point in time, whereas there may be a clear time differential between factors which exert their detrimental effect over a longer time period (e.g. high blood pressure and obesity) and those causing increased morbidity and mortality within a short time frame (e.g. malnutrition and overt cardiac failure $)^{21}$. Studies into dynamic risk assessment in relation to different time patterns are scarce in dialysis patients.

\section{Aim of the thesis}

The aim of the thesis is to assess the relationship between oscillatory and nonoscillatory time trends in selected treatment- and patient-related complications in dialysis patients. In particular the relationships between these time trends and patient outcomes, such as mortality and hospitalization are studied. The thesis is divided into two major parts, the first part (A) focuses on cyclical or oscillatory time trends; the second part (B) focuses on longitudinal, non- oscillatory, time trends. The chapters are followed by a general discussion. 


\section{(A) Complications in relation to oscillatory time patterns}

In general, in human physiology, two time patterns can be distinguished (i.e. oscillatory and longitudinal patterns) ${ }^{22}$. Oscillatory time patterns can be divided into ultradian (cycle time $<20$ hours), circadian (cycle time between 20 and 28 hours) and infradian (>28 hours) rhythms ${ }^{22}$. In this thesis, only circadian and infradian patterns, which usually follow a typical cyclical pattern are considered. Both may have an effect on pathophysiology in dialysis patients. Important circadian rhythms which could influence dialysis-related complications, such as intra-dialytic hypotension, are the patterns in BT and blood pressure. The relation between changes in BT and the hemodynamic response during dialysis was addressed earlier in this introduction. However, until present, no study has addressed the relationship between circadian changes in BT and the hemodynamic response during dialysis. This is the topic of Chapter 2.

Next to circadian changes, infradian oscillations also may have a large effect on (patho)physiological changes in dialysis patients. Earlier studies showed pronounced differences in both clinical and laboratory parameters between different seasons in dialysis patients ${ }^{23-25}$. The most pronounced seasonal differences were observed for blood pressure and for inter-dialytic weight gain ${ }^{23,25,26}$. Both are lower in the summer, possibly related to increased perspiration and insensible fluid loss. However, not all differences in blood pressure between seasons appear to be explained by volume factors. In the single study addressing this subject, which focused on peritoneal dialysis, no difference in extracellular volume assessed by bioimpedance was observed between seasons ${ }^{27}$, suggesting that volume-independent factors, such as differences in adrenergic or vascular activity also play a major role in the differences in blood pressure between seasons ${ }^{28}$.

Most studies have included small numbers of patients and/or were performed at single geographical location. The database of the Renal Research Institute (RRI) in New York, which includes historic information on more than 20,000 HD and 1,500 peritoneal dialysis patients treated between 2000 and 2012 in different locations in the United States (U.S.), provides an excellent opportunity to study the above mentioned phenomena in a far larger cohort, spread out over different geographical and climatological regions. This large cohort also provides the opportunity to assess seasonal differences in mortality, an important phenomenon which has not yet been studied in dialysis patients. This provides the background for Chapter 3, in which the effects of seasonal differences in clinical and laboratory parameters, and in mortality are assessed in a large cohort of HD patients. 


\section{(B) Longitudinal time patterns and complications in dialysis.}

In addition to effects related to oscillatory time patterns, longitudinal time patterns also may have an important relationship to variations in pathophysiology in dialysis patients. Certain distinguishable longitudinal patterns in patient parameters at the start of dialysis or before death or hospitalization may be a marker for patient wellbeing $^{21,22}$.

As mentioned above, clinical parameters, as well as risk factors for morbidity and mortality are usually studied by a cross sectional approach in dialysis patients, by which follow-up starts at the first date of dialysis and the outcome variable of interest is time until an event (usually death) occurs ${ }^{29}$. However, due to the fact that rapid changes in the clinical condition of the patient, as well as treatment-related factors may have profound effects on these parameters, a dynamic approach studying the relationship between risk factors versus morbidity and mortality appear to be warranted. This can easily be achieved in dialysis patients, since relevant clinical and laboratory parameters are assessed at frequent time intervals.

For a dynamic analysis of risk factors (and potentially relationship to outcomes), several approaches may be taken. Simple linear regression or linear mixed models are traditional approaches used to describe the behavior of variables over time. These models can provide a "slope" of the variable of interest over time. Combining these models with Cox proportional hazards models can be used to determine how "slope" in patient parameters is associated with outcomes. These approaches are used in Chapters $\mathbf{4}$ and $\mathbf{5}$. These approaches are similar to other joint modelling techniques of longitudinal and survival data ${ }^{17,19,30-36}$.

Other methods are used in Chapters 6, 7, and 8. First, the "forward process," in which the mean of expected values of various clinical or laboratory parameters after a certain event (e.g. start of dialysis) is computed. Second, the "backward process," where the mean expected value of the relevant parameters is analyzed in a retrograde manner with the event as the end point. Mean functions of the forward and backward processes are a necessity in the analysis of variables with much intraand inter-patient variability. A quintic spline approach to conditional means to estimate the mean function is used ${ }^{37,38}$.

In Chapter 4, the prognostic value of trends in blood pressure is assessed in HD patients in the RRI database. The hypothesis of this study is that both a decreasing trend in blood pressure, possibly resulting from myocardial failure, as well as an increasing trend, for instance related to volume overload, or progression of essential or secondary hypertension, could negatively influence prognosis in dialysis patients. In 
Chapter 5, the prognostic value of trends in BT is assessed. BT is an important physiological parameter. However, the relation between BT and outcome has not yet been studied in dialysis patients. The RRI database provides an excellent opportunity to assess this relationship, as data on BT are available for nearly all treatments.

Both low and high BTs could theoretically be related to outcome. An increase in BT could be due to infection, which is an important determinant for outcome in dialysis patients. On the other hand, in other diseases such as heart failure, a relationship between low BT and mortality was observed ${ }^{39}$. It has also been hypothesized that a reduction in $\mathrm{BT}$ might be an adaptive response of the body to inflammation when the body cannot cope with the increasing demands of fever on the body ${ }^{40}$. This might have parallels with the non-thyroidal illness syndrome, characterized by a lowering of the metabolic state and reduction of T3 levels ${ }^{41}$.

The dynamic backward approach, using a longitudinal data analysis backwards in time with the event as the common endpoint is applied in Chapters 6, 7, and 8. This part of the thesis is based on a previous study in the RRI cohort in which the dynamics of post-dialytic weight and serum albumin levels were assessed in deceased HD patients, in the 24 months prior the death of the patient ${ }^{29}$. In this study, using linear mixed models with linear spline function, both body weight as well as serum albumin decreased substantially three months before the death of the patients. Such an approach may facilitate the construction of clinical alert systems for future patients, which might facilitate earlier detection and intervention for patients at risk; it would also be of interest to investigate whether this approach would also be applicable for the prediction of hospitalization.

Hospitalization is very common in dialysis patients and has a significant economic impact and a greatly adverse impact on the quality of life. It has been estimated that approximately one-third of the total expenditure in ESRD patients results from hospital costs ${ }^{42}$. In the most recent USRDS survey, the mean yearly hospitalization rate in dialysis was estimated at 1.9 per patient year with a mean duration of hospital stay of 12 days per year. The main reasons for hospitalization were related to cardiovascular and infection-related causes. Hospitalized dialysis patients have an approximate 3-fold greater risk for in-hospital mortality as compared to hospitalized patients from the general population ${ }^{43}$.

Also striking are the high re-hospitalization rates, as $36 \%$ of hospitalized patients were re-admitted within 30 days after discharge ${ }^{2}$. The fact that many patients were rehospitalized for cardiovascular causes suggests part of this relation might be explained by fluid overload, possibly by insufficient adjustment of post dialysis target weight during dialysis despite a decline in lean body mass ${ }^{44}$. Detailed insight into the 
dynamics of clinical and laboratory parameters before a hospitalization episode, and in the relation between hospitalization and outcome is lacking in dialysis patients. Therefore, in Chapter 6, the dynamics of hospitalization rates before death in relation to changes in selected clinical and laboratory parameters are assessed in the RRI database, as well as the dynamic evolution of clinical and laboratory parameters before the first hospitalization episode.

The RRI database provides a great opportunity to perform detailed epidemiological studies in a U.S. dialysis population. However, due to the large impact of differences in ethnicity and treatment patterns on outcome ${ }^{45-50}$, it is of great relevance to study epidemiological patterns in a global population ${ }^{51-53}$. Recently, the MONDO (MONitoring of Dialysis Outcome) initiative was launched, an international consortium of HD providers from Southeast Asia, Argentina, Europe and the U.S. In the MONDO initiative, dialysis-specific data from different geographic regions can be analyzed at the patient-level. This allows consortium members to analyze relevant epidemiological trends in different geographical locations and in patients with different races and ethnicities, as well as in regions with different methods of treatment. In Chapter 7, the MONDO initiative is explained, and a descriptive analysis of selected clinical and laboratory parameters in the incident patients in different geographical locations is presented.

Thus, the MONDO initiative also provides a great opportunity to analyze whether the dynamics of relevant clinical and laboratory parameters can also be identified on a more global basis. In Chapter 8, the dynamics of systolic blood pressure, inter-dialytic weight gain, serum albumin and CRP levels before death are analyzed. These parameters were chosen because they reflect changes in three key domains, namely cardiovascular function, nutrition, and inflammation. In addition, a detailed analysis is presented on the dynamics of these parameters in different geographical regions in a global framework. 


\section{References}

1. Jain AK, Blake P, Cordy P, Garg AX. Global trends in rates of peritoneal dialysis. J Am Soc Nephrol. 2012;23: 533-44.

2. USRDS Annual data report. 2011.

3. Shaw C, Webb L, Casula A, Tomson CR. Chapter 4; Comorbidities and Current Smoking Status amongst Patients starting Renal Replacement Therapy in England, Wales and Northern Ireland from 2009 to 2010. Nephron Clin Pract. 2012;120 Suppl 1:c81-91.

4. Tattersall J, Martin-Malo A, Pedrini L, Basci A, Canaud B, Fouque D, Haage P, Konner K, Kooman J, Pizzarelli F, Tordoir J, Vennegoor M, Wanner C, ter Wee P, Vanholder R. EBPG guideline on dialysis strategies. Nephrol Dial Transplant. 2007;22 Suppl 2:ii5-21.

5. Lameire N, M.R., Complications of dialysis. 2000, New York: Marcel Dekker.

6. Mclntyre CW. Recurrent circulatory stress: the dark side of dialysis. Semin Dial. 2010;23:449-51.

7. Kooman J, Basci A, Pizzarelli F, Canaud B, Haage P, Fouque D, Konner K, Martin-Malo A, Pedrini L, Tattersall J, Tordoir J, Vennegoor M, Wanner C, ter Wee P, Vanholder R. EBPG guideline on haemodynamic instability. Nephrol Dial Transplant. 2007;22 Suppl 2:ii22-44.

8. Schneditz D, Rosales L, Kaufman AM, Kaysen G, Levin NW. Heat accumulation with relative blood volume decrease. Am J Kidney Dis. 2002;40:777-82.

9. van der Sande FM, Rosales LM, Brener Z, Kooman JP, Kuhlmann M, Handelman G, Greenwood RN, Carter M, Schneditz D, Leunissen KM, Levin NW. Effect of ultrafiltration on thermal variables, skin temperature, skin blood flow, and energy expenditure during ultrapure hemodialysis. J Am Soc Nephrol. 2005;16:1824-31.

10. Parfrey PS, Foley, RN.The clinical epidemiology of cardiac disease in chronic renal failure. J Am Soc Nephrol. 1999;10:1606-15.

11. Briet $M$, Pierre $B$, Laurent $S$, London GM. Arterial stiffness and pulse pressure in CKD and ESRD. Kidney Int. 2012;82:388-400.

12. Pecoits-Filho R, Lindholm B, Stenvinkel P. The malnutrition, inflammation, and atherosclerosis (MIA) syndrome -- the heart of the matter. Nephrol Dial Transplant. 2002;17 Suppl 11:28-31.

13. Ozkahya M, Ok E, Toz H, Asci G, Duman S, Basci A, Kose T, Dorhout Mees EJ. Long-term survival rates in haemodialysis patients treated with strict volume control. Nephrol Dial Transplant. 2006;21: 3506-13.

14. Ronco C, Kaushik M, Valle R, Aspromonte N, Peacock WF 4th. Diagnosis and management of fluid overload in heart failure and cardio-renal syndrome: the "5B" approach. Semin Nephrol. 2012;32: 129-41.

15. Wizemann V, Wabel P, Chamney P, Zaluska W, Moissl U, Rode C, Malecka-Masalska T, Marcelli D. The mortality risk of overhydration in haemodialysis patients. Nephrol Dial Transplant. 2009;24:1574-9.

16. Kovesdy CP, Kalantar-Zadeh K. Accuracy and limitations of the diagnosis of malnutrition in dialysis patients. Semin Dial. 2012;25:423-7.

17. Takahashi R, Ito $Y$, Takahashi $H$, Ishii H, Kasuga H, Mizuno M, Suzuki $Y$, Yuzawa $Y$, Maruyama S, Murohara T, Imai E, Matsuo S. Combined values of serum albumin, C-reactive protein and body mass index at dialysis initiation accurately predicts long-term mortality. Am J Nephrol. 2012;36:136-43.

18. Zager PG, Nikolic J, Brown RH, Campbell MA, Hunt WC, Peterson D, Van Stone J, Levey A, Meyer KB, Klag MJ, Johnson HK, Clark E, Sadler JH, Teredesai P. "U" curve association of blood pressure and mortality in hemodialysis patients. Medical Directors of Dialysis Clinic, Inc. Kidney Int. 1998;54:561-9.

19. Kalantar-Zadeh K, Streja E, Molnar MZ, Lukowsky LR, Krishnan M, Kovesdy CP, Greenland S. Mortality prediction by surrogates of body composition: an examination of the obesity paradox in hemodialysis patients using composite ranking score analysis. Am J Epidemiol. 2012;175:793-803.

20. Kalantar-Zadeh K, Regidor DL, Kovesdy CP, Van Wyck D, Bunnapradist S, Horwich TB, Fonarow GC. Fluid retention is associated with cardiovascular mortality in patients undergoing long-term hemodialysis. Circulation. 2009;119:671-9.

21. Kalantar-Zadeh K, Horwich TB, Oreopoulos A, Kovesdy CP, Younessi H, Anker SD, Morley JE. Risk factor paradox in wasting diseases. Curr Opin Clin Nutr Metab Care. 2007;10:433-42. 
22. Kooman JP, Usvyat L, van der Sande FM, Thijssen S, Levin N, Leunissen KM, Kotanko P. 'Time and Time Again': Oscillatory and Longitudinal Time Patterns in Dialysis Patients. Kidney Blood Press Res. 2012;35:534-48.

23. Argiles A, Mourad G, Mion C. Seasonal changes in blood pressure in patients with end-stage renal disease treated with hemodialysis. N Engl J Med. 1998;339:1364-70.

24. van der Sande FM, Kooman JP, Leunissen KM., Clinical implications of seasonal variations in hemodialysis patients. Blood Purif. 2008;26:193-5.

25. Cheung AK, Yan G, Greene T, Daugirdas JT, Dwyer JT, Levin NW, Ornt DB, Schulman G, Eknoyan G; Hemodialysis Study Group. Seasonal variations in clinical and laboratory variables among chronic hemodialysis patients. J Am Soc Nephrol. 2002;13:2345-52.

26. Sposito M, Nieto FJ, Ventura JE. Seasonal variations of blood pressure and overhydration in patients on chronic hemodialysis. Am J Kidney Dis. 2000;35:812-8.

27. Cheng LT, Jiang HY, Tang $\amalg$, Wang T. Seasonal variation in blood pressure of patients on continuous ambulatory peritoneal dialysis. Blood Purif. 2006;24:499-507.

28. Keatinge WR, Coleshaw SR, Cotter F, Mattock M, Murphy M, Chelliah R. Increases in platelet and red cell counts, blood viscosity, and arterial pressure during mild surface cooling: factors in mortality from coronary and cerebral thrombosis in winter. Br Med J (Clin Res Ed). 1984;289:1405-8.

29. Kotanko P, Thijssen S, Usvyat L, Tashman A, Kruse A, Huber C, Levin NW. Temporal evolution of clinical parameters before death in dialysis patients: a new concept. Blood Purif. 2009;27:38-47.

30. Hsieh F, Tseng YK, Wang JL. Joint modeling of survival and longitudinal data: likelihood approach revisited. Biometrics. 2006;62:1037-43.

31. Jacqmin-Gadda $H$, Thiebaut R, Dartigues JF. Joint modeling of quantitative longitudinal data and censored survival time. Rev Epidemiol Sante Publique. 2004;52:502-10.

32. Li N, Elashoff RM, Li G, Saver J. Joint modeling of longitudinal ordinal data and competing risks survival times and analysis of the NINDS rt-PA stroke trial. Stat Med. 2010;29:546-57.

33. Liu L. Joint modeling longitudinal semi-continuous data and survival, with application to longitudinal medical cost data. Stat Med. 2009;28:972-86.

34. Ratcliffe SJ, Guo W, Ten Have TR. Joint modeling of longitudinal and survival data via a common frailty. Biometrics. 2004;60:892-9.

35. Song $\mathrm{X}$, Wang $\mathrm{CY}$. Semiparametric approaches for joint modeling of longitudinal and survival data with time-varying coefficients. Biometrics. 2008;64:557-66.

36. Tapsoba Jde D, Lee SM, Wang CY. Joint modeling of survival time and longitudinal data with subjectspecific changepoints in the covariates. Stat Med. 2011;30:232-49.

37. Kurland BF, Heagerty PJ. Directly parameterized regression conditioning on being alive: analysis of longitudinal data truncated by deaths. Biostatistics. 2005;6:241-58.

38. Wang, Y., Smoothing Splines: Methods and Applications. 2011, New York: Champman and Hall.

39. Nallamothu BK, Payvar S, Wang Y, Kosiborod M, Masoudi FA, Havranek EP, Foody JM, Casscells SW, Krumholz HM. Admission body temperature and mortality in elderly patients hospitalized for heart failure. J Am Coll Cardiol. 2006;47:2563-4.

40. Romanovsky AA, Szekely M. Fever and hypothermia: two adaptive thermoregulatory responses to systemic inflammation. Med Hypotheses. 1998;50:219-26.

41. Koenig RJ. Modeling the nonthyroidal illness syndrome. Curr Opin Endocrinol Diabetes Obes. 2008;15:466-9.

42. Chan KE, Lazarus JM, Wingard RL, Hakim RM. Association between repeat hospitalization and early intervention in dialysis patients following hospital discharge. Kidney Int. 2009;76:331-41.

43. Daratha KB, Short RA, Corbett CF, Ring ME, Alicic R, Choka R, Tuttle KR. Risks of subsequent hospitalization and death in patients with kidney disease. Clin J Am Soc Nephrol. 2012;7:409-16.

44. Dalrymple LS, Mohammed SM, Mu Y, Johansen KL, Chertow GM, Grimes B, Kaysen GA, Nguyen DV. Risk of cardiovascular events after infection-related hospitalizations in older patients on dialysis. Clin J Am Soc Nephrol. 2011;6:1708-13.

45. Bradbury BD, Fissell RB, Albert JM, Anthony MS, Critchlow CW, Pisoni RL, Port FK, Gillespie BW. Predictors of early mortality among incident US hemodialysis patients in the Dialysis Outcomes and Practice Patterns Study (DOPPS). Clin J Am Soc Nephrol. 2007;2:89-99. 
46. Combe C, McCullough KP, Asano Y, Ginsberg N, Maroni BJ, Pifer TB. Kidney Disease Outcomes Quality Initiative (K/DOQI) and the Dialysis Outcomes and Practice Patterns Study (DOPPS): nutrition guidelines, indicators, and practices. Am J Kidney Dis. 2004.;44(5 Suppl 2):39-46.

47. Pifer TB, McCullough KP, Port FK, Goodkin DA, Maroni BJ, Held PJ, Young EW. Mortality risk in hemodialysis patients and changes in nutritional indicators: DOPPS. Kidney Int. 2002;62:2238-45.

48. Pisoni RL, Arrington CJ, Albert JM, Ethier J, Kimata N, Krishnan M, Rayner HC, Saito A, Sands JJ, Saran R, Gillespie B, Wolfe RA, Port FK. Facility hemodialysis vascular access use and mortality in countries participating in DOPPS: an instrumental variable analysis. Am J Kidney Dis. 2009;53:475-91.

49. Pisoni RL, Gillespie BW, Dickinson DM, Chen K, Kutner MH, Wolfe RA. The Dialysis Outcomes and Practice Patterns Study (DOPPS): design, data elements, and methodology. Am J Kidney Dis. 2004;44(5 Suppl 2):7-15.

50. Tentori F, Blayney MJ, Albert JM, Gillespie BW, Kerr PG, Bommer J, Young EW, Akizawa T, Akiba T, Pisoni RL, Robinson BM, Port FK. Mortality risk for dialysis patients with different levels of serum calcium, phosphorus, and PTH: the Dialysis Outcomes and Practice Patterns Study (DOPPS). Am J Kidney Dis. 2008;52:519-30.

51. Kawamoto K, Houlihan CA, Balas EA, Lobach DF. Improving clinical practice using clinical decision support systems: a systematic review of trials to identify features critical to success. BMJ. 2005;330:765.

52. Obama administration calls for electronic health records by 2015, in USA Today.

53. Krishnan M, Wilfehrt HM, Lacson E Jr. In Data We Trust: The Role and Utility of Dialysis Provider Databases in the Policy Process. Clin J Am Soc Nephrol. 2012;7:1891-6. 


\section{Part

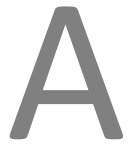

Oscillatory trends in dialysis patients and their outcomes 
$\overline{20}$ 


\section{Chapter 2}

Circadian variations in body temperature

during dialysis

Len A Usvyat, Peter Kotanko, Frank M van der Sande, Jeroen P Kooman, Mary Carter, Karel ML Leunissen, Nathan W Levin Nephrol Dial Transplant. 2012;27:1139-1144 


\section{Abstract}

Introduction

Thermal changes during dialysis strongly influence intra-dialytic hemodynamics. The mechanisms behind the increase in body temperature (BT) during hemodialysis (HD) are still not completely understood. The objective of this retrospective observational cohort study is to assess the effect of circadian variation on BT changes during HD, by comparing results in patients treated on different treatment shifts. Data from the Renal Research Institute (RRI) New York, clinical database encompassing patients treated in 6 states in the US were used. Data from January and August 2008 were used for analysis.

Results

BT changes during HD were categorized by dialysis shifts. Patients with morning shifts $(n=1064)$, afternoon shifts $(n=730)$ and evening shifts $(n=210)$ were compared. Pre-dialysis BT were significantly different among the different shifts (morning, $36.41\left[95 \% \mathrm{Cl}\right.$ : $36.39-36.43^{\circ} \mathrm{C}$; afternoon, 36.47 [36.45-36.49 ${ }^{\circ} \mathrm{C}$, evening, $36.67\left[36.64-36.70^{\circ} \mathrm{C}\right]$, respectively; $\mathrm{p}<0.001$ ). In August, but not in January, intra-dialytic increases in BT were significantly different between patients treated in morning $\left(0.07\left[0.058-0.082^{\circ} \mathrm{C}\right]\right)$, afternoon $(0.03$ $\left.\left[0.016-0.044^{\circ} \mathrm{C}\right]\right)$ and evening shifts $\left(-0.01\left[-0.032-0.012^{\circ} \mathrm{C}\right] ; \mathrm{p}<0.001 \mathrm{ANOVA}\right)$, although in January, treatment shift was a significant predictor of the intra-dialytic increase in BT. The intra-dialytic change in BT was related to the pre-dialysis $B T\left(r^{2}=0.31 ; p<0.001\right)$, but also to microbiological dialysate quality, treatment time and dialysate temperature. The intra-dialytic change in blood pressure was significantly related to changes in intra-dialytic BT irrespective of the study month.

Conclusions

Both pre-dialytic BT as well as changes in BT are significantly related to the timing of the dialysis shifts, in phase with the circadian BT rhythm. Due to the relation between BT changes and changes in intra-dialytic blood pressure, these findings might be of additional relevance in the pathogenesis of intra-dialytic hypotension. 


\section{Introduction}

Beginning with the seminal work of Maggiore et al, it is recognized that body temperature (BT) usually increases during hemodialysis (HD), which may be associated with unfavorable hemodynamic consequences ${ }^{1}$. The increase in BT during dialysis can be followed by cutaneous vasodilatation ${ }^{2}$, which has been explained as part of the body's thermoregulatory heat losing response to accumulated heat ${ }^{3,4}$. This cutaneous vasodilatation counteracts the normal hemodynamic response to a decline in blood volume, and thus may contribute to intra-dialytic hypotension. The rise in BT is especially pronounced with the prescription of dialysate temperatures above $37-37.5^{\circ} \mathrm{C}^{5,6}$. The use of cooler dialysate temperature improves vascular stability and might also reduce myocardial stunning during $\mathrm{HD}^{7}$. The beneficial hemodynamic effects of prescribing cooler dialysate temperature have been endorsed by recent guidelines $^{8}$.

The increase in BT during dialysis is still not completely understood. While energy transfer from dialysate to the patient undoubtedly contributes to the increase in BT during dialysis using dialysate temperature of $37.5^{\circ} \mathrm{C}, \mathrm{BT}$ during dialysis may even increase despite net energy loss from the patient to the extracorporeal system ${ }^{4-6,9}$. Various explanations for this phenomenon have been proposed, such as the production of cytokines during dialysis or an initial reduction in heat loss during dialysis due to peripheral vasoconstriction in response to hypovolemia, followed by sudden thermoregulatory vasodilatation in reaction to the accumulated heat ${ }^{10}$. This hypothesis is supported by the relation between ultrafiltration rate and energy transfer rate observed by Rosales et al. ${ }^{9}$. However, in a subsequent study, the changes in BT were not different when ultrapure dialysis was used with and without ultrafiltration ${ }^{11}$.

Thus, the causes of the increase in BT during dialysis are still not completely elucidated $^{5,12}$. A factor which has not received attention thus far is the effect of circadian temperature rhythm. Dialysis sessions often coincide with the normal rise in BT, with the lowest value at 4-4:30 a.m. and the highest at 6-7 p.m. ${ }^{13}$. If the circadian rhythm plays a role in the BT changes during dialysis, it is hypothesized that the change in BT would be highest during a morning shift and lowest during an evening shift.

The aim of the present study was to compare changes in BT between patients treated in different shifts, in order to assess the potential role of the circadian temperature rhythm in explaining thermal changes during dialysis. Moreover, the relation between the intra-dialytic blood pressure response and changes in BT was assessed. 


\section{Methods}

Utilizing the Renal Research Institute (RRI), New York, patient database, all pre- and post-dialysis BTs were collected. The timing of measurements was arbitrarily chosen in August 2008. To validate our findings on circadian differences, we also compared data on the same patients using treatment records from January 2008. Data were available for 11.2 [2.5] treatments during January 2008 and 11.3 [2.3] during August 2008 in each of 2004 patients. Only prevalent patients on dialysis were included (more than 90 days dialysis) and patients $>18$ years old.

Data at the start of the dialysis shift were available in all patients. Patients were divided according to the following classification: morning shift [start before 9:00 a.m.]; afternoon shift [start between 11.00 a.m. and 3:00 p.m.], and evening shift [start after 5:00 p.m.]. Data of patients starting during other time periods were not used. The mean of the different parameters during the monthly periods was used for analysis. Temperatures were recorded using an oral thermometer. While the majority of dialysis centers studied is located in New York State, some centers are located in Connecticut, North Carolina, Michigan, Illinois, and California.

If a shift change occurred during the month, the shift with the highest number of patient treatments was chosen and data were averaged only for that shift, and data on the other shifts were discarded. Only patients with the same shift and same facility in January and August were included. Otherwise, there were no exclusion criteria.

Comorbid conditions included those that were either in existence prior to the start of the study or those that were acquired during the study period. As a result, patients who developed an acute infection during the study period would be captured as having infection as one of the comorbid conditions. We admit that for conditions that are no longer in existence, difference clinics may have followed different methodology for documenting comorbid conditions.

Data on individual treatment prescriptions with regard to dialysate temperature are not available in the RRI database. However, most facilities have the same dialysate temperature for all its patients and all its shifts which were available. To take this into account, only facilities where more than $85 \%$ of patients were treated with the same dialysate temperature were included.

Dialysis temperature and composition was kept constant during the observation period. Clinics with patient-specific dialysate temperature prescription or with changes in dialysate temperature prescriptions were excluded. RRI follows ultrapure 
AAMI standards in water and dialysate quality. As such, all clinics studied achieve between 80 to $100 \%$ of the dialysate $L A L<0.03$ and cultures $<2$ cfu.

Two different devices to measure BT were used in this study. In New York, Connecticut and California, the Tempa Dot oral thermometer was used, whereas in Michigan, Illinois and North Carolina the Braun ear thermometer (WelchAllyn Type 6021) was used. Both methods comply to ASTM (American Society for Testing and Materials) requirements. Maximum error is within $0.2^{\circ} \mathrm{C}$ for the Braun thermometer and within $0.1^{\circ} \mathrm{C}$ for the Tempa Dot.

In order to assess the variation in pre-dialytic BT on a monthly basis using the two devices in this specific population, we calculated the coefficient of variation. For this, measurements on an individual basis (using the average of the January and August) were calculated for both devices. For the Tempa dot thermometer, a coefficient of variation (CV) of $0.0096(0.96 \%)$ was observed, whereas the CV was $0.0086(0.86 \%)$ for the Braun thermometer.

In order to correct for the type of thermometer (which was used on a state-wide basis) as well as for environmental temperatures, analysis was adjusted for treatment location.

All RRI patients are treated with Fresenius Optiflux high-flux dialyzers. Further, most treatments are done with blood flow of $400 \mathrm{ml} / \mathrm{min}$ and dialysate flow of $600 \mathrm{ml} / \mathrm{min}$. This analysis of the RRI database was approved by the Beth Israel Medical Center IRB.

\section{Data analysis}

The following parameters were analyzed: age, gender, race (classified as white, black and other), ethnicity (Hispanic and non-Hispanic), urea kinetic volume (V), pre-dialysis and post-dialysis $\mathrm{BT}$, change in $\mathrm{BT}$ during dialysis, pre-dialysis and post-dialysis systolic and diastolic blood pressures (SBP and DBP), change in BP during HD, residual renal function (RRF), microbiological dialysate quality, ultrafiltration volume, and presence of intra-dialytic events. Thermal parameters were compared between the different shifts by ANOVA and, if significant, differences between individual groups were further analyzed by posthoc analysis with Tukey correction. Determinants of predialysis BT and change in intra-dialytic BT were analyzed using linear multivariate analysis. Data are given in mean [95\% confidence intervals]. 


\section{Results}

\section{Demographics}

Both for January and August data from 1064 patients were available for the morning, from 730 for the afternoon and from 210 for the evening shifts. The mean dialysate temperature was $36.8\left[36.43-37.17^{\circ} \mathrm{C}\right]$. Patient characteristics are summarized in Table 2.1.

\section{Differences in thermal parameters between shifts}

Differences in pre-dialysis BT are shown in Table 2.2. Pre-dialysis BT was significantly different between the different shifts in August (morning, 36.41 [36.39-36.43 ${ }^{\circ} \mathrm{C}$; afternoon, $36.47\left[36.45-36.49^{\circ} \mathrm{C}\right]$, evening, $36.67\left[36.64-36.70^{\circ} \mathrm{C}\right]$, respectively; $\mathrm{p}<0.001)$. In January, pre-dialysis BT followed the same patterns and was also significantly different between morning and evening and afternoon and evening shifts (morning, $36.41\left[36.39-36.43^{\circ} \mathrm{C}\right]$; afternoon, $36.43\left[36.41-36.45^{\circ} \mathrm{C}\right]$, evening, 36.60 $\left[36.57-36.63^{\circ} \mathrm{C}\right]$, respectively; $\left.p<0.001\right)$.

\section{Determinants of pre-dialysis body temperature}

Using multivariate analysis, timing of dialysis shift, but also patient-related factors such as age, vintage, race, body mass index, co-morbidity, and location where the clinic is located were independent predictors of pre-dialysis BT of the patient (Table 2.3). BT was higher in black patients compared to white patients, lower in patients with comorbid conditions, and positively related to $\mathrm{BMI}$, whereas gender differences were not significant. $44 \%$ of the temperature variability was explained by the variables noted.

\section{Determinants of changes in body temperature}

Differences in changes in BT are shown in Table 2.2. Significant differences in pre- and post-dialysis BT were observed between the shifts in August and January, with the exception of morning and afternoon temperatures in January $[p<0.001$ ANOVA; $p<0.001$ between all different shifts using posthoc Tukey correction]. Intra-dialytic changes in BT were significant between shifts only in August (Table 2.2). The intradialytic change in BT was inversely related to the pre-dialysis BT of the patient $\left(r^{2}=0.28 ; p<0.001\right)$ (Figure 2.1). Pre-dialysis BT is significantly determined by patient shift as noted previously. 


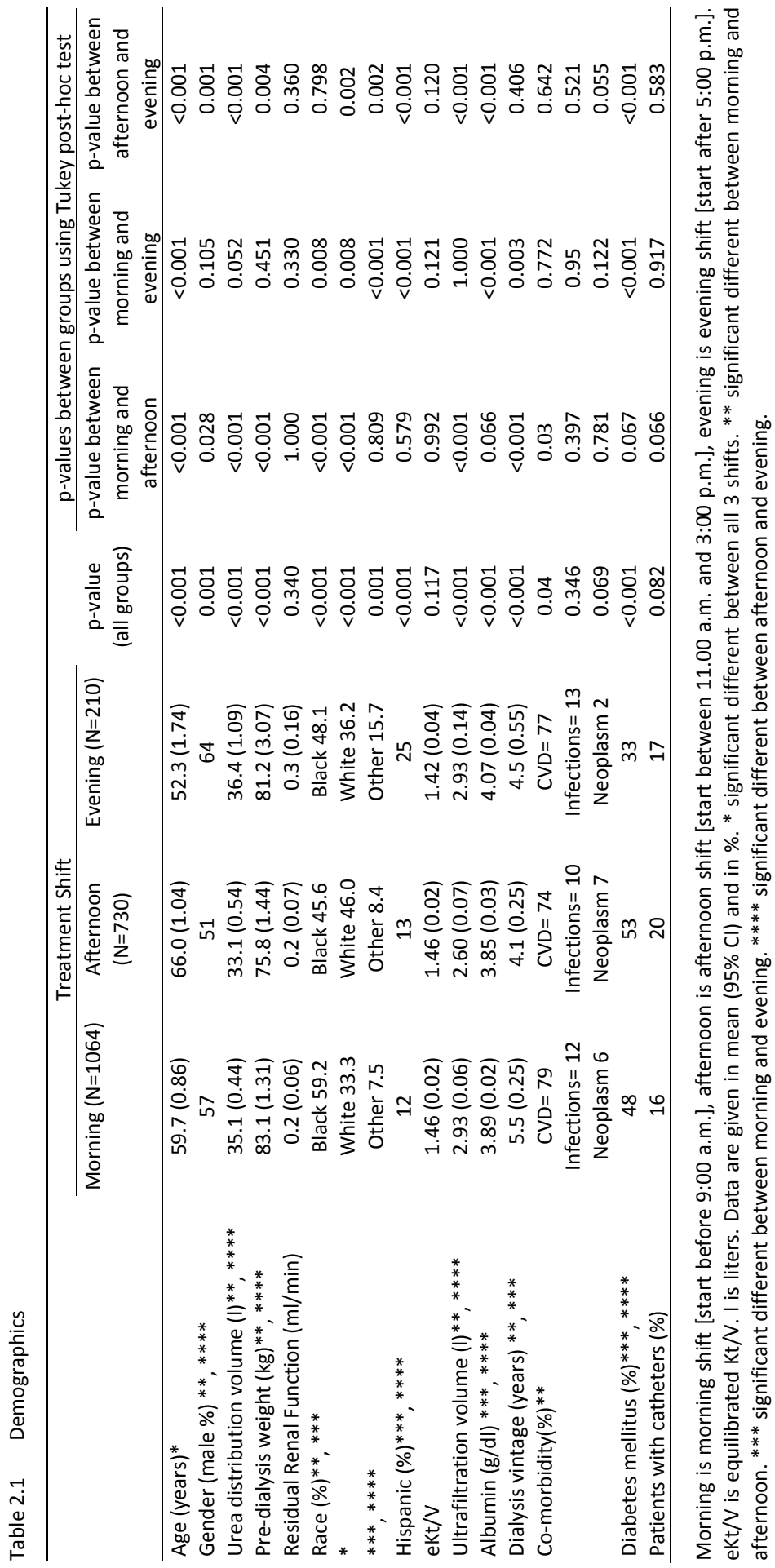




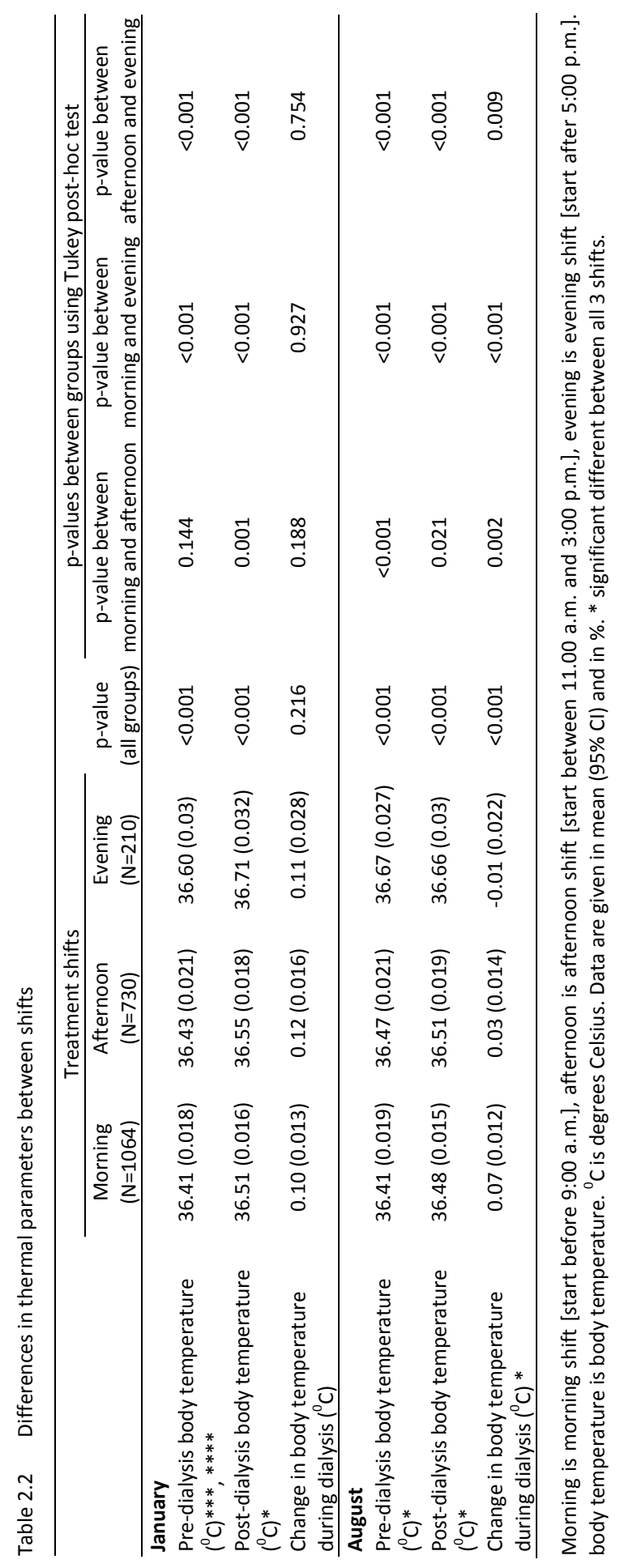




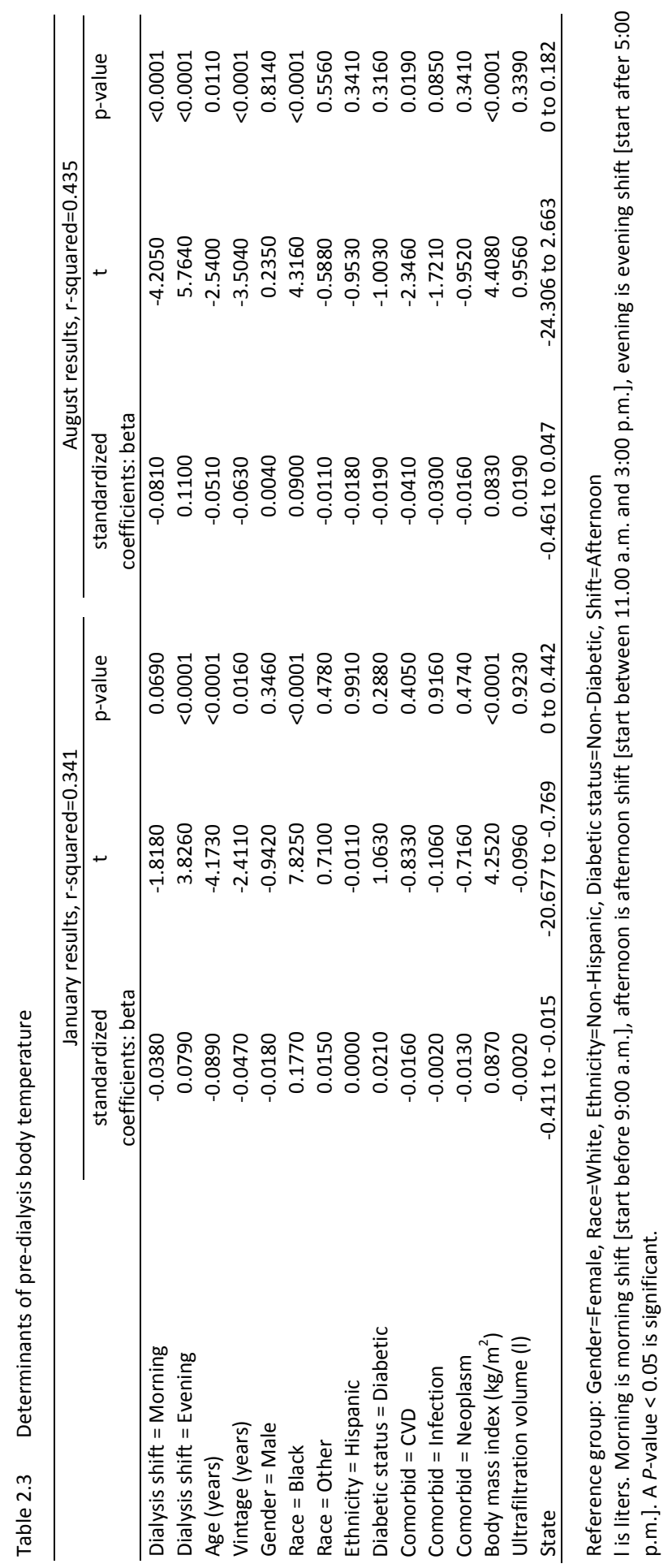


Pre-dialysis BT appeared to be a significant determinant of changes in BT during dialysis (Table 2.4), next to other treatment-related parameters such as treatment time, dialysate temperature, and microbiological dialysate quality. In January, treatment shift was a significant predictor for the intra-dialytic change in BT, but not in August, in which the effect of pre-dialytic BT appeared to overarch the effect of the shift (without inclusion of pre-dialysis BT, the shift was a significant predictor). $67 \%$ of the variation in changes in BT is not explained with the model.

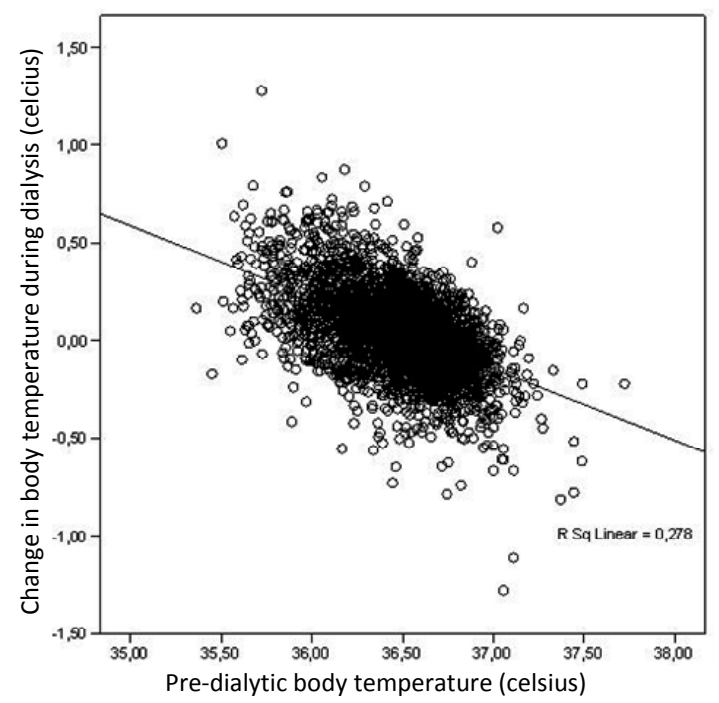

Figure 2.1 Relation between change in BT and pre-dialysis BT.

$\mathrm{Y}$-axis is change in $\mathrm{BT}$ during dialysis in ${ }^{\circ} \mathrm{C}$ (degrees Celsius), $\mathrm{x}$-axis is $\mathrm{BT}$ before dialysis in ${ }^{\circ} \mathrm{C}$ (degrees Celsius).

\section{Determinants of changes in blood pressure}

Pre-dialysis SBP was comparable between the 3 shifts. The $\%$ of treatments with intradialytic events were 25.6 [24.3-26.9] during the morning shift, 23.2 [22.0-24.4] during the afternoon, and 22.3 [20.0-24.6] during the evening shift $(p<0.05)$. The decline in SBP during HD was significantly higher in the morning shift compared to afternoon and evening shifts. In a multivariate analysis pre-dialysis BT was predictive of the decline in SBP $(\beta=-0.095, t=-3.7380, p=<0.001)$; Intra-dialytic change in BT was also predictive of the decline in SBP (Table 2.5). We acknowledge that $75 \%$ of the variation in changes in BP is not explained with the model. 


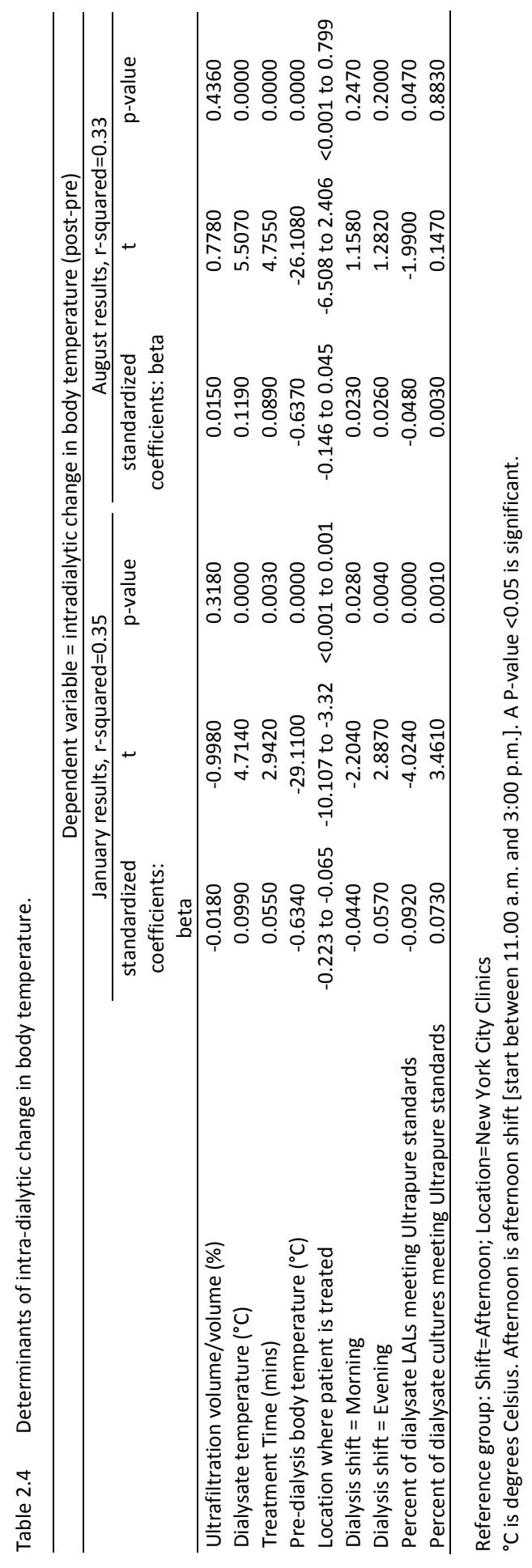




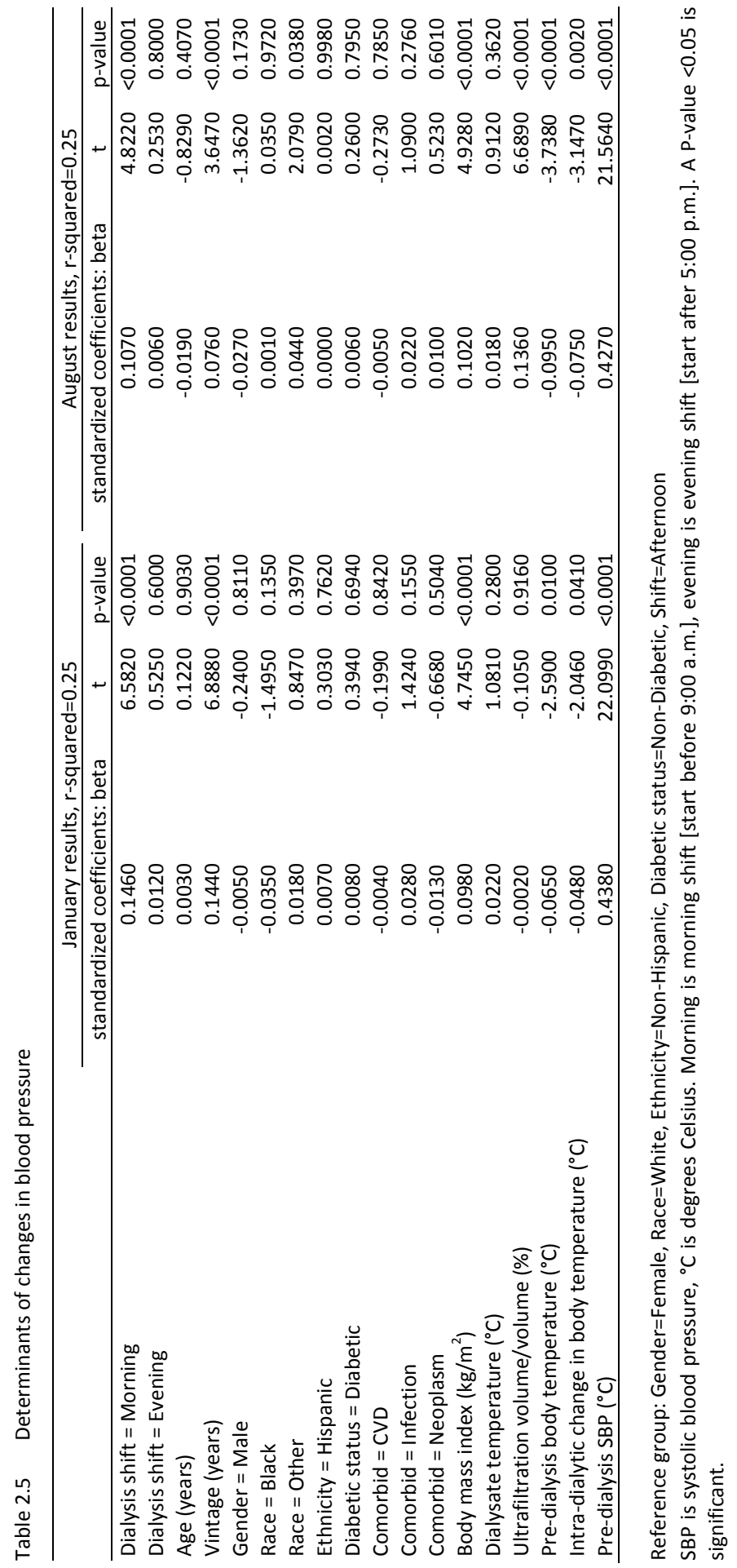




\section{Discussion}

The main finding of this study is the significant difference in the pre-dialysis BT and intra-dialytic BT rise between the morning, afternoon and evening shifts, which adds an additional explanation for the yet incompletely understood pathophysiology of BT changes during HD. This phenomenon may be explained by the circadian rhythm in BT. In phase with the normal circadian rhythm, BT increased during the morning and afternoon sessions, but remained stable during the evening sessions. The same trends in BT were observed both in January as well as in August, although the change in intradialytic BT was only significantly different between treatments in the latter month. However, in January, treatment shift remained a significant predictor for the intradialytic change in BT in multivariate analysis. Changes in BT were related to changes in intra-dialytic BP and thus might have pathophysiological significance.

Apart from circadian rhythm, other, patient-related, factors were also found to be related to pre-dialytic $\mathrm{BT}$, such as age, $\mathrm{BMI}$, and ethnicity, which is in agreement with literature in non-uremic patients ${ }^{14}$. In general, pre-dialysis BT was higher in black patients compared to whites, and inversely related to age. Moreover, pre-dialysis BT was significantly related also to BMI. Since the relationship between pre-dialytic BT and dialysate temperature is an important determinant for BT changes during dialysis ${ }^{5,12}$, these factors were indirectly related to the change in BT during dialysis.

Next to the timing of the dialysis shift and the patient-related factors influencing predialytic BT, also treatment-related factors, such as treatment time were related to the change in BT during dialysis. Ultrafiltration volume was not related to BT changes during dialysis. In contrast to the data of Rosales, this relationship was not significant, which may be due to the fact that, unlike the former study, BT or extracorporeal heat flow were not controlled in the present study ${ }^{9,10}$. In addition, also microbiological dialysate quality was a significant predictor for the intra-dialytic change in BT.

The clinical consequences of circadian changes in BT deserve consideration. In this study, the drop in BP was related to changes in BT during HD, after correction for confounders. However a causal relationship can not be implied from these observational data, as the selection of patients for different shifts might also be related to factors such as general health status, which could not be addressed in the present study. Previously, Fine et al. observed a strong relation between pre-dialysis BT of the patient and the beneficial effects of cool dialysis in the prevention of IDH ${ }^{12}$, showing the strongest protective effect in those patients who had the largest change in BT during standard dialysis temperatures. 
A limitation of this study is that comparison between individual patients assigned to different shifts over time could not be made. However, this may be offset by the large size of the database. Room temperature was not controlled or recorded in the present study. Interesting are the apparent differences in intra-dialytic temperature changes between August and January with respect to shift, which were significant in August but not in January. The reason for this observation cannot be deduced from the present data. However, ambient temperature might play a role, in the sense that in January pre-dialytic BT was lower as compared to August, and increased during dialysis also because of the effect of room temperature. Also, environmental temperature was not included in the analysis but the adjustment by treatment location takes this aspect partly into account. Other factors, such as food intake, might also have influenced thermal changes. However, in January treatment shift was also a significant predictor of the change in BT in multivariate analysis.

Changes in BT were in general limited and less pronounced compared to a previous study ${ }^{5}$, which is likely due to the fact that prescribed dialysate temperature in that report was $37.5^{\circ} \mathrm{C}$ as compared to $36.8\left[36.4-37.2^{\circ} \mathrm{C}\right.$ ] in this study. It is known ${ }^{4,15}$ that even small increases in BT during dialysis might affect the blood pressure response during dialysis. Therefore, the findings of the present study might support the use of an individualized prescription of dialysate temperature by means of isothermic dialysis ${ }^{16}$.

In conclusion, both the pre-dialytic BT as well as intra-dialytic changes in BT during HD are significantly related to the timing of the dialysis shifts. These differences appear to be in phase with the endogenous diurnal temperature rhythm, which provides an additional explanation for the increase in BT during dialysis. Due to the relation between changes in BT and the fall in blood pressure, this phenomenon might have pathophysiologic significance. 


\section{References}

1. Maggiore Q, Pizzarelli F, Sisca S, et al.. Blood temperature and vascular stability during hemodialysis and hemofiltration. Trans Am Soc Artif Intern Organs. 1982;28:523-7.

2. Kooman JP, Gladziwa U, Bocker G, van Bortel LM, van Hooff JP, Leunissen KM. Role of the venous system in hemodynamics during ultrafiltration and bicarbonate dialysis. Kidney Int. 1992;42:718-26.

3. Schneditz D, Levin NW. Keep your temper: how to avoid heat accumulation in haemodialysis. Nephrol Dial Transplant. 2001;16:7-9.

4. van der Sande FM, Gladziwa U, Kooman JP, Bocker G, Leunissen KML. Energy transfer is the single most important factor for the difference in vascular response between isolated ultrafiltration and hemodialysis. J Am Soc Nephrol. 2000;11:1512-7.

5. Van der Sande FM, Kooman JP, Burema JH, et al.. Effect of dialysate temperature on energy balance during hemodialysis: quantification of extracorporeal energy transfer. Am J Kidney Dis. 1999;33: 1115-21.

6. Provenzano R, Sawaya B, Frinak S, et al.. The effect of cooled dialysate on thermal energy balance in hemodialysis patients. ASAIO Trans. 1988;34:515-8.

7. Selby NM, Burton JO, Chesterton U, McIntyre CW. Dialysis-induced regional left ventricular dysfunction is ameliorated by cooling the dialysate. Clin J Am Soc Nephrol. 2006;1:1216-25.

8. Kooman J, Basci A, Pizzarelli F, et al. EBPG guideline on haemodynamic instability. Nephrol Dial Transplant. 2007;22 (Suppl 2):ii22-44.

9. Rosales LM, Schneditz D, Morris AT, Rahmati S, Levin NW. Isothermic hemodialysis and ultrafiltration. Am J Kidney Dis. 2000;36:353-61.

10. Gotch FA, Keen ML, Yarian SR An analysis of thermal regulation in hemodialysis with one and three compartment models. ASAIO Trans. 1989;35:622-4.

11. Van der Sande FM, Rosales LM, Brener Z, et al. Effect of ultrafiltration on thermal variables, skin temperature, skin blood flow, and energy expenditure during ultrapure hemodialysis. J Am Soc Nephrol. 2005;16:1824-31.

12. Fine A, Penner B. The protective effect of cool temperature dialysate is dependent on patients' predialysis temperature. Am J Kidney Dis. 1996;28:262-5.

13. Kelly G. Body temperature variability (Part 1): a review of the history of body temperature and its variability due to site selection, biological rhythms, fitness, and aging. Altern Med Rev. 2006;11: 278-93.

14. Kelly GS. Body temperature variability (Part 2): masking influences of body temperature variability and a review of body temperature variability in disease. Altern Med Rev. 2007;12:49-62.

15. Aoki K, Stephens DP, Saad AR, Johnson JM. Cutaneous vasoconstrictor response to whole body skin cooling is altered by time of day. J Appl Physiol. 2003;94:930-4.

16. Maggiore $Q$, Pizzarelli F, Santoro A, et al. The effects of control of thermal balance on vascular stability in hemodialysis patients: results of the European randomized clinical trial. Am J Kidney Dis. 2002;40:280-90. 


\section{Chapter 3}

Seasonal variations in mortality, clinical and laboratory parameters in hemodialysis patients:

a five year cohort study

Len A Usvyat, Mary Carter, Stephan Thijssen, Jeroen P Kooman, Frank M van der Sande, Paul Zabetakis, Paul Balter, Nathan W Levin, Peter Kotanko

Clin J Am Soc Nephrol. 2012;7:108-115. 


\section{Abstract}

\section{Background}

Mortality varies seasonally in the general population, but it is unknown whether this phenomenon is also present in hemodialysis (HD) patients with known higher background mortality and emphasis on cardiovascular causes of death. The aim of the present study was to assess seasonal variations in mortality, in relation to clinical and laboratory variables in a large cohort of chronic HD patients over a five year period.

\section{Methods}

15,056 patients were studied of 51 Renal Research Institute clinics from six states of varying climate in the USA. Seasonal differences were assessed by chi-square test, univariate and multivariate cosinor analysis.

Results

Mortality, both all-cause and cardiovascular, was significantly higher during winter as compared to other seasons (14.2 deaths per 100 patient years in winter, 13.1 in spring, 12.3 in autumn, and 11.9 in summer). The increase in mortality in winter was more pronounced in younger patients, as well as in whites and in males. Seasonal variations were similar across climatologically different regions. Seasonal variations were also observed in neutrophil/lymphocyte ratio, serum calcium, potassium, and platelet values. Differences in mortality disappeared when adjusted for seasonally variable clinical parameters.

\section{Conclusions}

In a large cohort of dialysis patients, significant seasonal variations in overall and cardiovascular mortality were observed, which were consistent over different climatic regions. Other physiological and laboratory parameters were also seasonally different. We observed that mortality differences were related to seasonality of physiological and laboratory parameters. Seasonal variations should be taken into account when designing and interpreting longitudinal studies in dialysis patients. 


\section{Introduction}

Seasonal variations have a large influence on physiology. Dietary intake, body composition and physical activity, and immune function are known to follow a seasonal rhythm ${ }^{1}$. Seasonal variations in mortality have been described in the general population, which were largely attributable to a higher incidence of cardiovascular complications ${ }^{2}$. In addition, the incidence of cardiovascular and respiratory diseases is higher in winter.

While various laboratory and blood pressure measurements in dialysis patients have also shown seasonal variations ${ }^{3-5}$, seasonal fluctuations in mortality have not been studied. Due to the far higher background mortality of dialysis patients as compared to the general population, it is questionable whether the more subtle seasonal variations could still be observed in such patients. On the other hand, most dialysis patients die from cardiovascular causes ${ }^{2}$. However, the spectrum of cardiovascular diseases in the general population, in whom a significant seasonal variability has been observed for ischemic heart disease and cerebrovascular disease, is different from that of dialysis patients. In the latter, relative incidence of acute cardiac death is much higher, and the relative incidence of thrombo-embolic processes much lower than in the general population ${ }^{6}$. Insight into seasonal variations of mortality, as well as in other physiologic and laboratory parameters might be relevant for interpretation and design of studies in dialysis patients. In addition, it is not known whether geographical variables associated with different climates are also related to variations in mortality and clinical and laboratory parameters in dialysis patients.

The aim of the study was to assess over a five-year period seasonal differences in mortality, as well as in physiological and laboratory parameters, in a large cohort of dialysis patients in different climate regions of the United States (US).

\section{Study population and methods}

\section{Data sources and study population}

We conducted a retrospective record review of chronic in-center hemodialysis (HD) patients treated in 51 Renal Research Institute (RRI) and New York Dialysis Services clinics between April 1, 2004 and March 31, 2009. For the analysis of mortality, open cohort of patients was included: any chronic HD patient who was treated in RRI during this time period was included $(N=15,056)$. The cause of death is documented in the clinical database using ICD-9 codes. 
If a patient dies within 30 days from withdrawal from one of RRI's clinics, it is company's policy to track the death in the clinical database. For those patients, cause of death is still documented in the system based on the death record; withdrawal is not classified as a cause of death.

Seasons were defined according to the meteorological definition winter as December through February, spring as March through May, summer as June through August, autumn as September through November ${ }^{7}$. All cause, as well as cardiovascular versus non-cardiovascular mortality was noted per season per year and expressed as deaths per 100 patient years.

RRI dialysis clinics, located in six states, were grouped in distinct major climate groups: ${ }^{8}$ Continental $(14,015$ pts in New York, Connecticut, Michigan, Illinois), Mediterranean (316 pts in coastal California), and Subtropical (737 pts in North Carolina). For the mortality analysis, any patient with even one treatment was included in order to exclude survival bias of prevalent patients. We used the classification system of ERA-EDTA which separates patients into groups of $<75$ and $\geq 75$ years old.

For the analysis of other parameters, 10,303 (table 3.1) patients in the RRI database with at least one treatment in each of the four calendar seasons were included (this was done to assure that patients contribute to more than one season). Pre-dialysis and post-dialysis systolic blood pressures (SBP) were recorded each treatment and were averaged over each month and season. Intra-dialytic change in SBP was defined as the difference between pre-dialysis and post-dialysis pressures.

Laboratory results in the RRI database were automatically downloaded from Spectra Laboratories (Rockleigh, NJ). Data on blood pressure, body temperature, and weight was recorded by the clinic staff and cleaning procedures were followed to correct any clear data entry errors.

This analysis of the RRI database was approved by the Beth Israel Medical Center IRB.

\section{Statistical analysis}

Chi-square tests were used to compare mortality rates between seasons. Univariate cosinor analysis was conducted to test for seasonality in mortality and other variables ${ }^{9}$. For seasonal variations in mortality all values are presented with $95 \%$ confidence intervals [lower limit - upper limit]. For seasonal variations in inter-dialytic weight gain and blood pressure all values are presented with $95 \%$ confidence intervals $[+95 \% \mathrm{Cl}]$. 
All p-values are two-sided. The analyses were conducted in SPSS version 18 (IBM, Somers, NY) and SAS version 9.2 (SAS Institute, Cary, NC).

Table 3.1 Patient Characteristics

\begin{tabular}{|c|c|c|c|c|}
\hline & \multirow[b]{2}{*}{ All Patients } & \multicolumn{3}{|c|}{ Mediterranea } \\
\hline & & Continental & $\mathrm{n}$ & Subtropical \\
\hline Number of patients & 10303 & 9605 & 226 & 472 \\
\hline \multicolumn{5}{|l|}{ Demographics } \\
\hline Black (percent) & $49 \%$ & $49 \%$ & $2 \%$ & $64 \%$ \\
\hline White (percent) & $42 \%$ & $41 \%$ & $84 \%$ & $34 \%$ \\
\hline Male (percent) & $55 \%$ & $55 \%$ & $56 \%$ & $53 \%$ \\
\hline Diabetic (percent) & $49 \%$ & $48 \%$ & $41 \%$ & $55 \%$ \\
\hline Age (years) & $61.7(15.4)$ & $61.7(15.3)$ & $68.2(14.9)$ & $59.3(16.1)$ \\
\hline Vintage (years) & $3.2(3.5)$ & $3.2(3.5)$ & $2.8(3.3)$ & $3.3(3.5)$ \\
\hline \multicolumn{5}{|l|}{ Hemodialysis data } \\
\hline Pre dialysis systolic blood pressure (mmHg) & $149.2(17.6)$ & $149.2(17.5)$ & $145.3(18.2)$ & $150(18.9)$ \\
\hline Pre dialysis diastolic blood pressure (mmHg) & $77.7(11)$ & $77.8(11)$ & $74(10.6)$ & $78.9(11.5)$ \\
\hline Pre dialysis weight $(\mathrm{kg})$ & $78.6(21)$ & 78.5 (20.9) & $76(20.4)$ & $80.6(22.1)$ \\
\hline Pre dialysis temperature $\left({ }^{\circ} \mathrm{C}\right)$ & $36.5(0.2)$ & $36.5(0.2)$ & $36.4(0.2)$ & $36.1(0.2)$ \\
\hline Inter-dialytic weight gain (kg) & $2.7(0.9)$ & $2.7(0.9)$ & $2.3(1)$ & $2.9(1)$ \\
\hline Pre-post dialysis systolic blood pressure $(\mathrm{mmHg})$ & $10.2(10.8)$ & $10.1(10.7)$ & $9.1(10.9)$ & $11.8(11.6)$ \\
\hline Pre-post dialysis temperature $\left({ }^{\circ} \mathrm{C}\right)$ & $-0.1(0.1)$ & $-0.1(0.1)$ & $0(0.1)$ & $-0.1(0.1)$ \\
\hline \multicolumn{5}{|l|}{ Plasma measures } \\
\hline Albumin (g/dl) & $3.8(0.4)$ & $3.8(0.4)$ & $3.8(0.3)$ & $3.8(0.4)$ \\
\hline Hemoglobin (g/dl) & $11.9(0.7)$ & $11.8(0.7)$ & $11.9(0.6)$ & $12(0.7)$ \\
\hline Neutrophil to lymphocyte ratio & $3.9(2.5)$ & $3.9(2.5)$ & $4.1(2.2)$ & $3.8(2.5)$ \\
\hline Phosphorus (mg/dl) & $5.2(1.1)$ & $5.2(1.1)$ & $5.1(1.2)$ & $5.2(1.2)$ \\
\hline Calcium (mg/dl) & $9(0.6)$ & $9(0.6)$ & $9(0.5)$ & $8.9(0.5)$ \\
\hline Platelet $(103 / \mu l)$ & $221(70.8)$ & 221.4 (70.9) & $128.3(54.8)$ & $217.8(69.2)$ \\
\hline Ferritin (ng/dl) & $673.5(457.7)$ & $673.6(463.5)$ & $575.3(343.2)$ & $719.5(373.5)$ \\
\hline Potassium (mEq/l) & $4.8(0.5)$ & $4.8(0.5)$ & $5(0.5)$ & $4.6(0.5)$ \\
\hline
\end{tabular}

The data are presented as the means (SD)

\section{Cosinor analysis}

Cosinor analysis is a statistical methodology that fits a cosine function to a time series. It is often used in the analysis of biologic time series that demonstrate predictable rhythms ${ }^{10}$. Cosine functions have the following characteristics ${ }^{11}$ graphically shown in (Figure 3.1). 


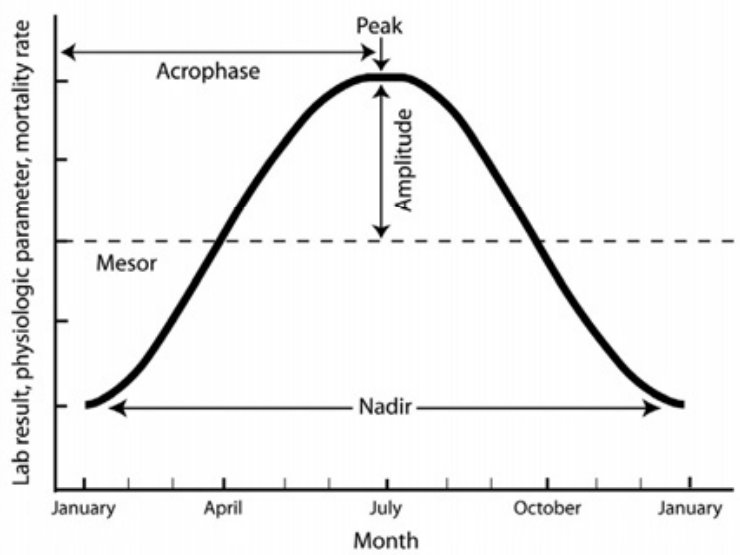

- Mesor: estimate of central tendency of the distribution of values

- Peak: the point of culmination of an oscillatory function

- Nadir: lowest value of an oscillatory function

- Amplitude: difference between the peak and the mean value of a wave

- Acrophase: time at which the peak of a rhythm occurs

Figure 3.1 Cosinor characteristics. Mesor, estimate of central tendency of the distribution of values; peak, the point of culmination of an oscillary function; nadir, lowest value of an oscillatory function; amplitude, difference between the peak and the mean value of a wave; acrophase, time at which the peak of a rhythm occurs.

In general, a cosine function of the following form was fitted for each variable of interest $^{12}$.

$y=M+a \cos \left(\frac{2 \pi t}{T}+\theta\right)+\varepsilon$

where $y$ is the variable of interest (for example, mortality rate per season, mean predialysis systolic blood pressure per season, mean albumin per season); $T$ is the fundamental time period during which the cycle occurs (in the current study cyclical patterns over a course of one year or four seasons); $M$ is the mesor or the mean value over the year; $a$ is amplitude; $t$ is the time period for which the value is fitted (for example, summer or spring); $\theta$ is the acrophase; and $\varepsilon$ is the error term.

Using a simple trigonometric identity one can transform the relationship above to a linear regression model as follows:

$y=M+A \cos \left(\frac{2 \pi t}{T}\right)+B \sin \left(\frac{2 \pi t}{T}\right)$ 
Where $A=a \cos (\theta)$ and $B=-a \sin (\theta)$.

For every year and season, one can calculate $\cos \left(\frac{2 \pi t}{T}\right)$ and $\sin \left(\frac{2 \pi t}{T}\right)$ terms.

Using simple linear regression coefficients $A$ and $B$ can be estimated. Significant coefficients of $A$ and $B$ suggests that there exists a cyclical relationship to the variables of interest. In this analysis, this suggests that variables of interest follow a seasonal pattern. Predicted values presented herewith are those predicted by the linear model above; actual values are the mean values per season.

\section{Results}

We studied 15,056 in-center HD patients for the mortality analysis (open cohort of patients) and 10,303 in-center HD patients for the analysis of physiological and laboratory parameters. The majority of the population was male $(55 \%)$ with a mean age of 61.7 years and mean vintage at start of study of 3.2 years. Black patients accounted for $49 \%$ of the population and white patients accounted for $42 \%$. Diabetic patients comprised $49 \%$ of the population.

For the mortality analysis, patients were followed for anywhere between 1 day and 4.9 years with a median follow up of 13.8 months.

\section{Seasonal variations in mortality}

All cause mortality was highest in winter $(14.2[95 \% \mathrm{Cl} ; 13.3$ to 15.1$]$ deaths/100 patient years), followed by spring (13.1 [12.2 to 14.0] deaths/100 patient years), and autumn (12.3 [11.4 to 13.2 ] deaths/100 patient years), with lowest mortality in summer (11.9 [11.1 to 12.7 ] deaths/100 patient years) (Figure 3.2). Cosinor analysis over a five year period demonstrated clearly a seasonal component of mortality. The results were confirmed by comparing seasonal variations across three climate zones with highest mortality in winter.

In the continental climate clinics, there were significant differences between mortality rates between all four seasons $(p<0.05)$; in the subtropical climate clinics, there were significant differences between winter and summer as well as winter and fall $(p<0.05)$; in the mediterranean climate, while summer mortality rate was lower than winter, the only significant differences were between winter and fall (this may be attributed to small number of deaths and patients in the mediterranean climate). 


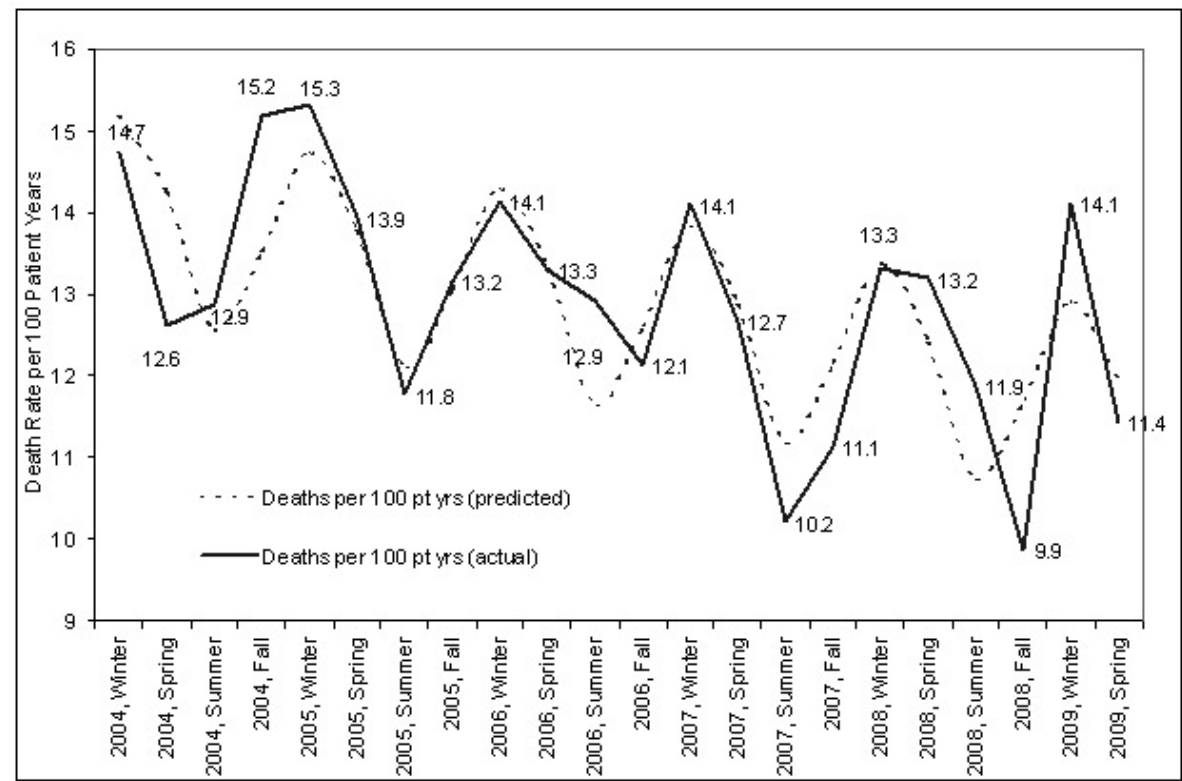

Figure 3.2 Seasonal variations in mortality. The figure demonstrates seasonal aspects of mortality rate calculated in deaths per 100 patient years calculated per season and year. Pt yrs, patientyears.

Using linear regression transformation to cosinor analysis, coefficients for seasonal factors (mesor, amplitude, acrophase) were significantly different from zero. Highest mortality (peak) was in winter and lowest mortality was in summer (nadir). Overall mortality declined over a five year period by an average of 0.46 deaths per 100 patient years $(p<0.01)$.

In subgroup analysis, the mortality during the winter period (compared to all other seasons) was significantly higher in males, in patients below 75 years of age, in whites (compared to summer and autumn) and in diabetic patients (compared to summer and autumn) (Table 3.2).

The main cause of death in the population was cardiovascular disease, which was significantly higher in the winter period (compared to all other seasons) for all climates. Death rates for infection-related reasons were highest in autumn but the differences were not significantly different across seasons (this may be attributed to a relatively small number of deaths: $<90$ deaths in each season) (Table 3.3). 
Table 3.2 Seasonal variations in mortality by demographic groups (deaths per 100 patient years).

\begin{tabular}{|c|c|c|c|c|}
\hline & Winter & Spring & Summer & Autumn \\
\hline \multicolumn{5}{|l|}{ Gender } \\
\hline Male & $14.87 *, * *, * * *$ & 12.92 & 11.92 & 11.78 \\
\hline Female & 13.47 & 13.22 & 11.97 & 12.88 \\
\hline \multicolumn{5}{|l|}{ Race } \\
\hline Black & 11.64 & 10.31 & 10.20 & 10.18 \\
\hline White & $18.6 * *, * * *$ & 17.77 & 14.84 & 15.71 \\
\hline Other & 11.01 & 9.98 & 10.21 & 10.07 \\
\hline \multicolumn{5}{|c|}{ Diabetic status } \\
\hline No & $12.47^{* *}$ & 11.68 & 10.84 & 11.09 \\
\hline Yes & $16.25^{* *}, * * *$ & 14.65 & 13.22 & 13.64 \\
\hline \multicolumn{5}{|l|}{ Age } \\
\hline$<75$ years & $11.29 *, * *, * * *$ & 9.98 & 9.08 & 9.74 \\
\hline$\geq 75$ years & 26.08 & 25.60 & 23.59 & 22.52 \\
\hline
\end{tabular}

${ }^{*}=$ Winter vs. Spring, $\mathrm{p}<0.05 .{ }^{* *}=$ Winter vs. Summer, $\mathrm{p}<0.05 .{ }^{* * *}=$ Winter vs. Autumn, $\mathrm{p}<0.05$

Table 3.3 Seasonal variations in mortality by causes of death (deaths per 100 patient years).

\begin{tabular}{llccc}
\hline & \multicolumn{1}{c}{ Winter } & Spring & Summer & Autumn \\
\hline Cardiovascular & $8.74^{*}, *, * * *$ & 7.48 & 7.16 & 7.05 \\
Infection & 1.23 & 0.95 & 1.01 & 1.29 \\
Neoplasm & 0.45 & 0.44 & 0.28 & 0.27 \\
\hline
\end{tabular}

${ }^{*}=$ Winter vs. Spring, $\mathrm{p}<0.05{ }^{* *}=$ Winter vs. Summer, $\mathrm{p}<0.05 .{ }^{* * *}=$ Winter vs. Autumn, $\mathrm{p}<0.05$

Patient physiological and laboratory parameters are not confounders to this cosinor analysis of mortality as they are in the hypothesized causal pathway between seasons and mortality ${ }^{13}$. In order to understand whether it is the seasonality in patient physiological and laboratory parameters that affect the seasonality in mortality, we analyzed whether mortality remains seasonally significant when adjusting by interdialytic weight gain, pre-dialysis blood pressure, intra-dialytic change in blood pressure, neutrophil-to-lymphocyte ratio, platelet count, and calcium. After these adjustments, season itself was no longer a significant predictor of mortality.

\section{Seasonal variations in inter-dialytic weight gain and blood pressure}

Cosinor analysis over a five year period demonstrated a seasonal component for predialysis SBP with higher blood pressure in the winter months. Coefficients for seasonal factors (mesor, amplitude, acrophase) were significantly different from zero $(p<0.01)$ (Figure 3.3). The largest intra-dialytic drop in SBP occurred in winter and was smallest in summer (Figure 3.4). 


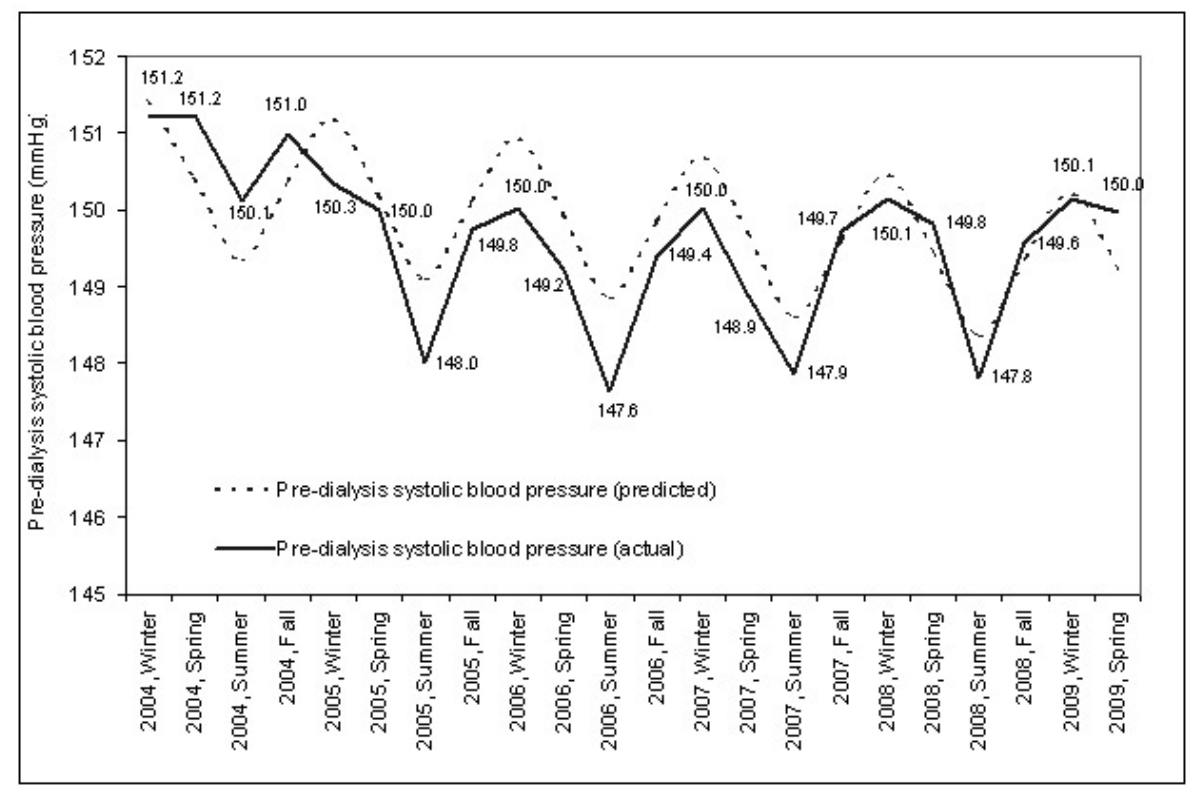

Figure 3.3 Seasonal variations in pre-dialysis SBP. The figure demonstrates seasonal aspects of predialysis SBP calculated as mean pre-dialysis SBP by season and year in $\mathrm{mmHg}$.

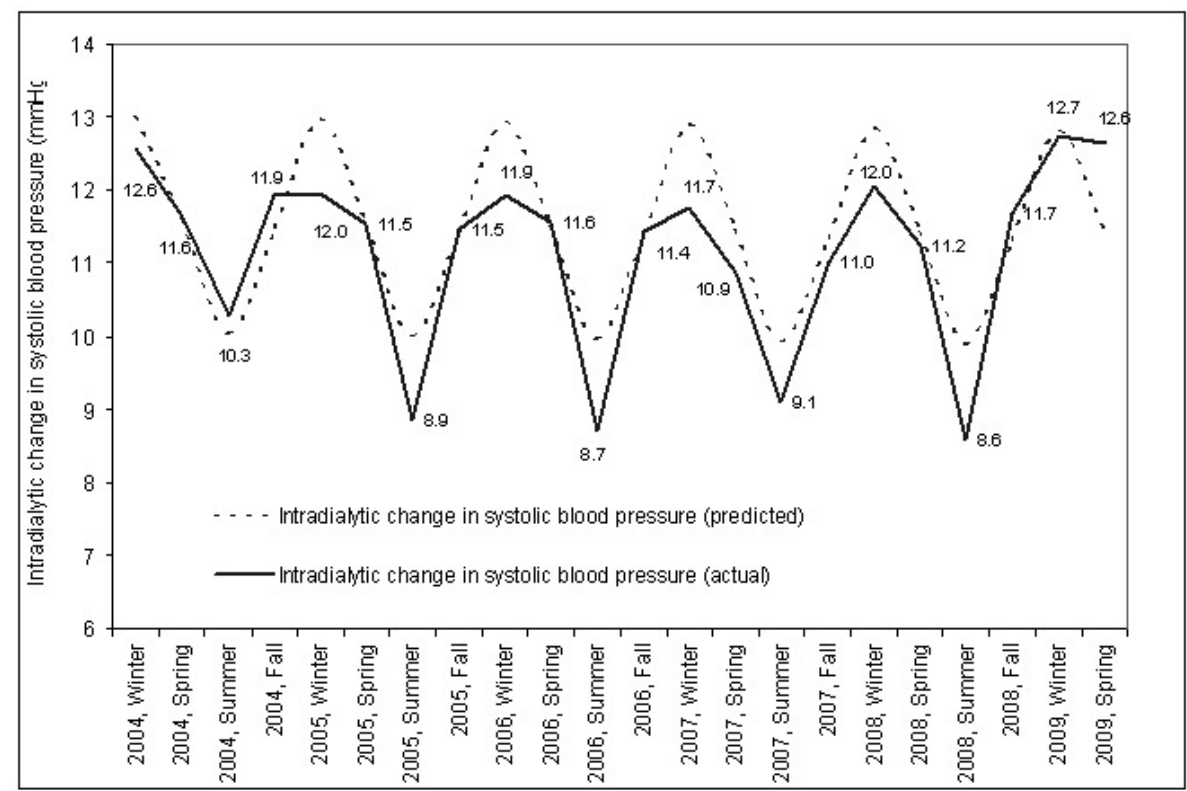

Figure 3.4 Seasonal variations in intra-dialytic change in SBP. The figure demonstrates seasonal aspects of intra-dialytic change in SBP calculated as mean intra-dialytic change in SBP by season and year in $\mathrm{mmHg}$. 
Across all three climatic zones, pre-dialysis SBP (in $\mathrm{mmHg}$ ) was also highest in the winter (continental (150.4 [0.15]), mediterranean (146.3 [1.08]), subtropical (152.03 [0.72]) and lowest in the summer: continental (148.4 [0.15]), mediterranean (143.2 [1.04]), subtropical (151.0 [0.74]). Inter-dialytic weight gain followed a cyclical pattern (Figure 3.5) with highest weight gains in the winter and lowest in the summer $(p<0.01)$.

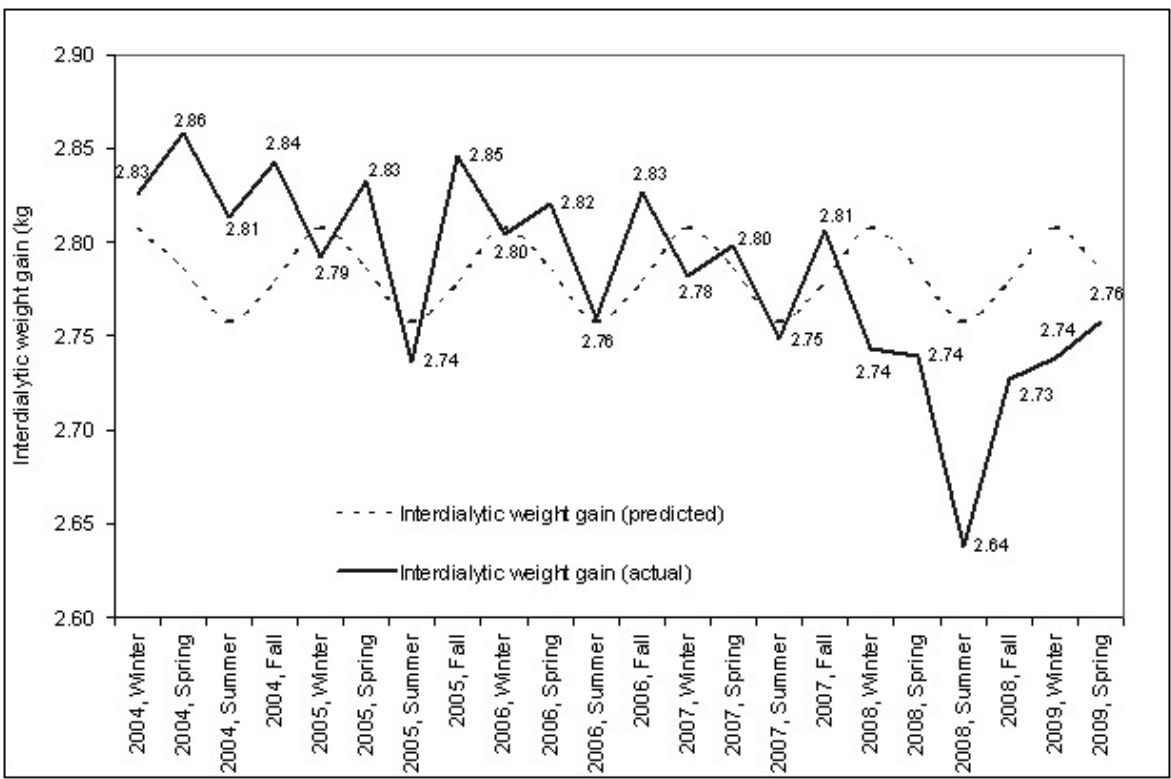

Figure 3.5 Seasonal variations in inter-dialytic weight gain. The figure demonstrates seasonal aspects of inter-dialytic weight gain calculated as mean inter-dialytic weight gain by season and year in kilograms.

\section{Seasonal variations in laboratory and dialysis adequacy parameters}

Cosinor analysis over a five year period demonstrated a seasonal component of neutrophil to lymphocyte ratio, serum potassium, and platelet count with highest values in winter and lowest in summer. Highest serum calcium values were observed in autumn and lowest in spring (data not shown). Coefficients of seasonal factors (mesor, amplitude, acrophase) were different from zero $(p<0.01)$. No seasonal pattern was noted for albumin, hemoglobin, phosphate and ferritin. 


\section{Discussion}

The main results of the present study are the large seasonal variations in mortality with highest values for the winter. Seasonal variation in various clinical and laboratory parameters was observed also. Mortality differences between seasons are no longer significant when adjusting for seasonally variable clinical and laboratory parameters. This finding suggests that the relation between season and mortality is either mediated by the variation in risk factors or by an underlying factor (e.g intercurrent disease), which is seasonally variable and affects both risk factors as well as mortality.

Variations in mortality have been described in the general population, but not previously in dialysis patients. Despite milder winters, comparable differences in seasonal mortality were also apparent in coastal California, which is in agreement with European observations in the general population where seasonal differences in mortality were even higher in Mediterranean countries than in Scandinavian countries $^{2,13-15}$. Still, due to the relatively small number of patients in climate zones other than continental, these data should be interpreted with some caution.

Although, due to the higher background mortality, absolute differences in mortality are much larger in the present study as compared to studies in the general population, relative differences appeared to be comparable. In this study, relative mortality was $19 \%$ higher in winter as compared to the summer period, which is comparable to the differences in myocardial infarction in the study of Crawford in the general population ${ }^{16}$. In this study, the seasonal amplitude of standardized mortality rates fell in general between 10 and $25 \%$.

The higher mortality in winter was largely due to cardiovascular causes. This is in agreement with data from the general population, in which mortality from cardiovascular diseases such as cardiac arrhythmias, strokes, and ischemic heart disease is the largest contributory factor to winter mortality ${ }^{1,2}$. Possible explanations advanced for this phenomenon in the general population are hemoconcentration and an increased tendency for thrombosis ${ }^{13,16}$, although it remains to be determined whether this phenomenon would also play a role in dialysis patients. Other factors, such as variations in sympathetic nervous system activity, might also contribute to an increased propensity for arrhythmias ${ }^{17,18}$, although in non-uremic subjects no seasonal variations in catecholamine excretion have been observed ${ }^{19}$.

Although in the general population mortality from infectious disease was higher in the winter ${ }^{2}$, no significant differences between seasons were observed in the present study, which is likely due to the low rate of death from this complication in the database. It cannot be excluded that infections themselves lead to cardiovascular 
complications and subsequent mortality. Neutrophil-lymphocyte ratio, a surrogate marker of inflammation, was higher in winter period for all climates. In earlier studies, higher neutrophil count and lower lymphocyte count were related to mortality in chronic dialysis patients ${ }^{20}$.

Pre-dialytic blood pressure, as well as intra-dialytic drop in blood pressure was highest in the winter period for all climates. In several studies on dialysis patients seasonal variations in blood pressure have been observed ${ }^{3,4,21}$, Fine et al. did not observe seasonal variations in blood pressure in normotensive treated patients of a Canadian dialysis centre despite large differences in outside temperatures, and suggested that blood pressure variations in European studies might be related to non-climatic factors $^{22}$. However, our results confirm the results of the study by Cheung in the HEMO study population in a North American population ${ }^{3}$. Admittedly, unlike Fine et al, neither in the present nor in the HEMO data a distinction was made between patients treated with or without antihypertensive agents. Ultrafiltration volume was lowest in summer and highest in the winter period winter period which is in agreement with other studies in which seasonal variations in blood pressure and inter-dialytic weight gain were observed $3,23,24$. However, in other studies no relation between variations in extracellular volume and seasonal variations in blood pressure were observed $^{21}$.

Of laboratory parameters, serum potassium showed a seasonal variation with highest values in winter, in agreement with Yanai ${ }^{5}$, which might appear somewhat counterintuitive in view of a higher purposed fruit intake during summer times ${ }^{25}$. Although this might reflect a higher dietary intake in winter ${ }^{1}$, phosphate levels were not significantly different between the various seasons in our study. No reliable data on phosphate binders were available in our study. In general, seasonal variations in laboratory parameters are not completely consistent between studies ${ }^{3,5}$.

Limitations of the study are the fact that several relevant laboratory parameters were not included in the database and the fact that patients in the different climate regions in the US were unevenly distributed in the data. Data on blood pressure, body temperature, and weight was recorded by the clinic staff and, while some human error component is possible, additional data cleaning procedures were followed to assure that errors in the data entry were accounted for. If there are any remaining data entry errors they are expected to be random and not systematic. Vitamin D levels which have been previously related to seasonal variability in blood pressure ${ }^{26}$ and perhaps even mortality ${ }^{27}$, were not systematically measured.

Strong points of the present study are the inclusion of a large number of dialysis patients, which makes it by far the largest dialysis cohort in which the concept of 
seasonal variations has been studied, and that this is the first study reflecting seasonal differences in mortality as well as risk factors for mortality, in this population. An increased understanding of the impact of these variations may be useful for future interventional trials.

In conclusion, in a large cohort of dialysis patients, both overall and cardiovascular mortality were significantly higher in winter as compared with other seasons. This variation was consistent in different climate regions. The relative increase in mortality appeared to be most pronounced in younger patients, in whites and in males. Various physiological and laboratory parameters were also seasonally different and were related to the seasonality in mortality. This study provides additional knowledge on the variation of the mortality risks of dialysis patients. Seasonal variations should be taken into account when designing and interpreting longitudinal studies in dialysis patients. 


\section{References}

1. Shephard RJ, Aoyagi Y. Seasonal variations in physical activity and implications for human health. Eur J Appl Physiol. 2009;107:251-71.

2. The Eurowinter Group. Cold exposure and winter mortality from ischaemic heart disease, cerebrovascular disease, respiratory disease and all causes in warm and cold regions of Europe. Lancet. 1997;349:1341-6.

3. Cheung AK, Yan G, Greene T, Daugirdas JT, Dwyer JT, Levin NW, Ornt DB, Schulman G, Eknoyan G,; Hemodialysis Study Group. Seasonal variations in clinical and laboratory variables among chronic hemodialysis patients. J Am Soc Nephrol. 2002;13:2345-52.

4. Argiles A, Mourad G, Mion C. Seasonal changes in blood pressure in patients with end-stage renal disease treated with hemodialysis. N Engl J Med. 1998;339:1364-70.

5. Yanai M, Satomura A, Uehara Y, Murakawa M, Takeuchi M, Kumasaka K. Circannual rhythm of laboratory test parameters among chronic haemodialysis patients. Blood Purif. 2008;26:196-203.

6. Meier $P$, Vogt $P$, Blanc $E$. Ventricular arrhythmias and sudden cardiac death in end-stage renal disease patients on chronic hemodialysis. Nephron. 2001;87:199-214.

7. American Meteorological Society: classification of seasons. http://amsglossary.allenpress.com/ glossary/search?id=winter1

8. Köppen climate classification. Encyclopædia Britannica. Encyclopædia Britannica Online. Encyclopædia Britannica, 2011. Web. 02 Jul. 2011.

9. Moen AN, Boomer GS. A tandem cosine algorithm for modeling rhythmic change. Ecological Modelling. 2000;134:275-82.

10. Barnett Adrian, Dobson Annette. Analysing Seasonal Health Data, 2010

11. O'Dell LE, Chen SA, Smith RT, Specio SE, Balster RL, Paterson NE, Markou A, Zorrilla EP, Koob GF. Extended access to nicotine self-administration leads to dependence: Circadian measures, withdrawal measures, and extinction behavior in rats. J Pharmacol Exp Ther. 2007;320:180-93.

12. Padhye N, Hanneman S. Cosinor Analysis for Temperature Time Series Data of Long Duration. Biol Res Nurs. 2007;9:30.

13. Keatinge WR, Donaldson GC. The impact of global warming on health and mortality. South Med J. 2004;97:1093-9.

14. Mercer JB. Cold--an underrated risk factor for health. Environ Res. 2003;92:8-13.

15. Grut M. Cold related death in some developed countries. Lancet. 1987;1:212.

16. Crawford VL, McCann M, Stout RW. Changes in seasonal deaths for myocardial infarction. Q J Med. 2003;96:45-52.

17. Fries RP, Heisel AG, Jung JK, Schieffer HJ. Circannual variation of malignant ventricular tachyarrhythmias in patients with implantable cardioverter-defibrillators and either coronary artery disease or idiopathic dilated cardiomyopathy. Am J Cardiol. 1997;79:1194-7.

18. Anand K, Aryana A, Cloutier D, Hee T, Esterbrooks D, Mooss AN, Mohiuddin SM. Circadian, daily, and seasonal distributions of ventricular tachyarrhythmias in patients with implantable cardioverterdefibrillators. Am J Cardiol. 2007;100:1134-8.

19. Nicolau GY, Haus E, Popescu M, Sackett-Lundeen L, Petrescu E. Circadian, weekly, and seasonal variations in cardiac mortality, blood pressure, and catecholamine excretion. Chronobiology International. 1991;8:149-59.

20. Reddan DN, Klassen PS, Szzech LA. White blood cells as a novel mortality predictor in hemodialysis patients. Nephrol Dial Transplant. 2003;18:1167-73.

21. Cheng LT, Jiang HY, Tang $\amalg$, Wang T. Seasonal variation in blood pressure of patients on continuous ambulatory peritoneal dialysis. Blood Purif. 2006;24:499-507.

22. Fine A. Lack of seasonal variation in blood pressure in patients on hemodialysis in a North American center. Am J Kidney Dis. 2000;36:562-5.

23. Sposito M, Nieto FJ, Ventura JE. Seasonal variations of blood pressure and overhydration in patients on chronic hemodialysis. Am J Kidney Dis. 2000;35:812-8.

24. Argilés A, Lorho R, Servel MF, Chong G, Kerr PG, Mourad G. Seasonal modifications in blood pressure are mainly related to interdialytic body weight gain in dialysis patients. Kidney Int. 2004;65:1795-801. 
Chapter 3

25. Cox BD, Whichelow MJ, Prevost AT. Seasonal consumption of salad vegetables and fresh fruit in relation to the development of cardiovascular disease and cancer. Public Health Nutrition. 1999;3: 19-29.

26. Argiles A. Lorho R, Servel MF, Couret I, Chong G, Mourad G. Blood pressure is correlated with vitamin d(3) serum levels in dialysis patients. Blood Purif. 2002;20:370-5.

27. Wolf M, Shah A, Gutierrez O, Ankers E, Monroy M, Tamez H, Steele D, Chang Y, Camargo CA Jr, Tonelli $M$, Thadhani R. Vitamin D levels and early mortality among incident hemodialysis patients. Kidney International. 2007;72:1004-13. 


\section{Part

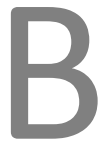

Longitudinal, non-oscillatory, trends in dialysis patients 
54 


\section{Chapter 4}

Blood pressure stability in hemodialysis patients confers a survival advantage: results from a large retrospective cohort study

Jochen G Raimann*, Len A Usvyat*, Stephan Thijssen, Peter Kotanko, John Rogus, Eduardo Lacson Jr, Nathan W Levin *Both authors contibuted equally Kidney Int. 2012;81:548-558 


\section{Abstract}

The association between changes in systolic blood pressure (SBP) and diastolic blood pressure (DBP), and the use of cardioprotective drugs on survival of incident HD patients, was examined in this retrospective cohort study. Pre-HD SBP and DBP were averaged over the first month of HD. Slopes, reflecting temporal changes, were computed by linear regression of SBP and Cox regression was used for survival analyses. Patients were initially stratified into four cohorts (below 120, 120 to 150, 151 to 180, and above $180 \mathrm{mmHg}$ ) and further subdivided into groups with stable (no more than a 1-mmHg change per month), increasing (over 1-mmHg per month), and decreasing (less than 1-mmHg per month) slopes during the first year. Analyses were repeated for patients who were treated with cardioprotective drugs for 1 month or more in the second year. In 10,245 patients (59\% prescribed cardioprotective drugs), both increases and decreases in all ranges of blood pressure (BP) were associated with worse outcomes, whereas stable BP had a survival advantage at all levels of SBP and DBP. Use of cardioprotective drugs attenuated changes and improved survival. Validation and sensitivity analyses confirmed the primary findings. Therefore, previous temporal trends need to be considered in patient care, and the use of cardioprotective agents is associated with enhanced survival at all BP levels. 


\section{Introduction}

In the general population a strong association exists between elevated systolic blood pressure (SBP) and diastolic blood pressure (DBP) and mortality ${ }^{1}$. A recent metaanalysis showed that lower SBP and DBP are associated with a lower risk for stroke and ischemic heart disease at all ages ${ }^{2}$.

In the majority of dialysis patients pre-dialysis blood pressure (BP) measurements are above normal ranges ${ }^{3,4}$. The Kidney Disease: Improving Global Outcomes (KDIGO) reviewed current views and concluded hypertension to be ubiquitous in this population and suggested the general use of cardioprotective drugs (CPD) ${ }^{5}$. Kidney Disease Outcomes Quality Initiative (KDOQI) recommends without evidence from RCTs BP goals below 140/90 before and $130 / 80 \mathrm{mmHg}$ after hemodialysis (HD) ${ }^{6}$.

Similar to the general population ${ }^{7-15}$, in hemodialysis patients the relation of BP and mortality is U-shaped ${ }^{16-18}$. Deviations of BP levels associated with favorable outcomes in HD patients as compared to those in the general population have not been satisfactorily explained. It is remarkable that most studies considered the patients' average BP level as a prognosticator, ignoring trends over time and the potential impact of changes in BP.

This observational cohort study (www.clinicaltrials.gov: \#NCT01330004) examines the evolution and temporal changes in SBP and DBP of incident patients over the first year of dialysis and repeated the analysis in the subsequent year for survivors to test the hypothesis that both increasing and decreasing BPs are associated with higher mortality. The secondary aim of the study was to test the hypothesis that use of CPD influences BP trends and is associated with better survival.

In the general population a strong association exists between elevated SBP and DBP and mortality ${ }^{1}$. A recent meta-analysis showed that lower SBP and DBP are associated with a lower risk for stroke and ischemic heart disease at all ages ${ }^{2}$.

In the majority of dialysis patients pre-dialysis BP measurements are above normal ranges $^{3,4}$. The KDIGO reviewed current views and concluded hypertension to be ubiquitous in this population and suggested the general use of cardioprotective drugs $(C P D)^{5}$. KDOQI recommends without evidence from RCTs BP goals below 140/90

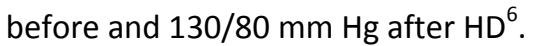

As in the general population ${ }^{7-15}$, in HD patients the relation of BP and mortality is Ushaped $^{16-18}$. Deviations of BP levels associated with favorable outcomes in HD patients as compared to those in the general population have not been satisfactorily explained. It is remarkable that most studies considered the patients' average BP level 
as a prognosticator, ignoring trends over time and the potential impact of changes in BP.

This observational cohort study (www.clinicaltrials.gov: \#NCT01330004) examines the evolution and temporal changes in SBP and DBP of incident patients over the first year of dialysis and repeated the analysis in the subsequent year for survivors to test the hypothesis that both increasing and decreasing BPs are associated with higher mortality. The secondary aim of the study was to test the hypothesis that use of CPD influences BP trends and is associated with better survival.

\section{Methods}

\section{Patient Population}

All patients starting in-center HD between January $1^{\text {st }}, 2000$ and December $31^{\text {st }}, 2008$ in 51 facilities (see Appendix) operated by Renal Research Institute (RRI) and New York Dialysis Services were included in this analysis (www.clinicaltrials.gov: \#NCT01330004). Patients with complete demographic data (date of birth, gender, race, ethnicity, diabetic status, and height), at least one routine laboratory measurement, at least 13 treatments in the first year of dialysis, and the last treatment occurring within 30 days prior to discharge date or the end of year 1 , were included. Patients were monitored during Year 1; for patients who survived Year 1 the observation time was extended to the end of Year 2. Patients who were transferred to other clinics, switched modality, and received a transplant were censored for survival analysis. This observational study was approved by the Institutional Review Board of Beth Israel Medical Center, New York.

\section{Cohorts of Systolic and Diastolic Blood Pressure (SBP, DBP)}

SBP and DBP were measured prior to HD in a sitting position after a five minutes rest period with an oscillometric method by healthcare professionals in the clinics. Average SBP and DBP were individually computed from all treatments during the first 30 days after dialysis initiation. Slopes of SBP and DBP change were determined by univariable linear regression (LR) using all BP measurements available in Year 1 until censoring or death.

Patients were stratified into four groups according to mean pre-dialysis SBP during the first month as follows: 1 ) below 120, 2) between 120 and 150, 3) between 151 and 180, and 4) above $180 \mathrm{mmHg}$. They were further stratified based on the slope of SBP as stable (SBP slope between +1 and $-1 \mathrm{mmHg}$ per month), decreasing (less than 
$-1 \mathrm{mmHg}$ per month) or increasing (more than $+1 \mathrm{mmHg}$ per month). This four-bythree grouping strategy resulted in 12 analytical cohorts.

In a second analysis, patients were stratified according to mean pre dialysis DBP in the first 30 days into: 1) below 65, 2) between 65 and 75, 3) between 76 and 85, and 4) above $85 \mathrm{mmHg}$. DBP categorization according to slope was done in the same manner as for SBP.

\section{Clinical parameters}

Pre- and post-dialysis weight and inter-dialytic weight gain (IDWG), were averaged from all available treatments during Year 1 . Albumin was measured using the bromocresol green method, performed by a certified laboratory (Spectra Laboratories, Rockleigh, NJ, USA). Comorbid illnesses were classified according to the International Classification of Diseases (ICD) ${ }^{19}$.

\section{Evaluation of Cardioprotective Drugs}

Angiotensin converting enzyme inhibitors, angiotensin receptor blockers and betaadrenergic blockers were identified as $\mathrm{CPD}^{5,20,21}$. Therapy with CPD was binary coded and based on whether a patient was prescribed either of these drugs at start or during Year 1 of dialysis, regardless of dose and frequency.

\section{Sensitivity analyses}

Three sensitivity analyses were conducted to exclude confounding influences. A first sensitivity analysis defined a slope between 0.03 and $-0.03 \mathrm{mmHg}$ as stable and included patients with increasing and declining slopes only when showing a $p$-value below 0.05 for the slope in univariable LR. Others were entirely excluded. A second sensitivity analysis excluded first and last six SBP measurements per patient. This approach aimed to exclude confounding of slope computation by outliers at the beginning and the end of the observation period. A third sensitivity analysis excluded the lowest and highest quartiles of increasing and decreasing SBP, respectively. This aimed to exclude the possibility that the results were driven by a small number of patients with large changes in SBP. A fourth sensitivity analysis defined the categories of SBP change as follows: patients with increasing SBP were only those whose a lower $95 \%$ confidence interval limit was larger than $1 \mathrm{mmHg}$, and patients with decreasing SBP were only those with an upper 95\% confidence interval limit below $-1 \mathrm{mmHg}$. Patients with stable SBP were only those with upper 95\% confidence interval limits lower than $1 \mathrm{mmHg}$ and lower $95 \%$ confidence interval limit larger than $-1 \mathrm{mmHg}$. This analysis aimed to test the statistical validity of our approach. 


\section{Validation analyses}

In 2007 RRI instituted a quality initiative recommending physicians to prescribe CPD to most patients. To support the analysis of potential impact of CPD on SBP and DBP slope and to minimize 'treatment by indication' bias, analyses of BP levels and slope were compared between the subset of patients who: 1) initiated dialysis prior to 2006 and were not on CPD, and 2) Patients who started dialysis after the quality initiative in 2007 and were on CPD. The effect of this quality initiative on survival was confirmed in the two subset with Kaplan Meier Analysis to compare death rates between patients with and without CPD therapy.

To exclude influences of seasonality, the survival analysis was repeated with adjustment for season of death (Spring, Summer, Autumn and Winter) for patients who died and season of end of Year 1 for patients who survived.

To validate findings in Year 1, all analyses were repeated in patients who survived more than one month with more than 13 treatments in Year 2. Clinical parameters were determined anew at the beginning of Year 2 .

To validate our findings are related to the changes in SBP and not by other causes of death, the analysis of the relation of temporal changes of SBP at all levels of SBP to hazard ratio was repeated for cardiovascular mortality only.

\section{Statistical analysis}

Slopes of SBP and DBP were computed using univariable LR. Multivariable LR Models for patients with increasing and decreasing SBP slopes as dependent variables were constructed to estimate the effect of CPD on SBP change. Cox Proportional Hazards Models were developed to determine the relation of SBP and DBP slope to survival. A p-value below 0.05 was considered significant. Analyses were conducted in SPSS 18.0 (IBM, Somers, NY) and SAS 9.2 (SAS Institute, Cary, NC).

\section{Results}

\section{Demographics}

The study population included 12695 incident HD patients, 10245 patients (Figure 4.1) who underwent on average $94 \pm 37$ treatments in Year 1 were analyzed. Demographics are shown in Table 4.1. 


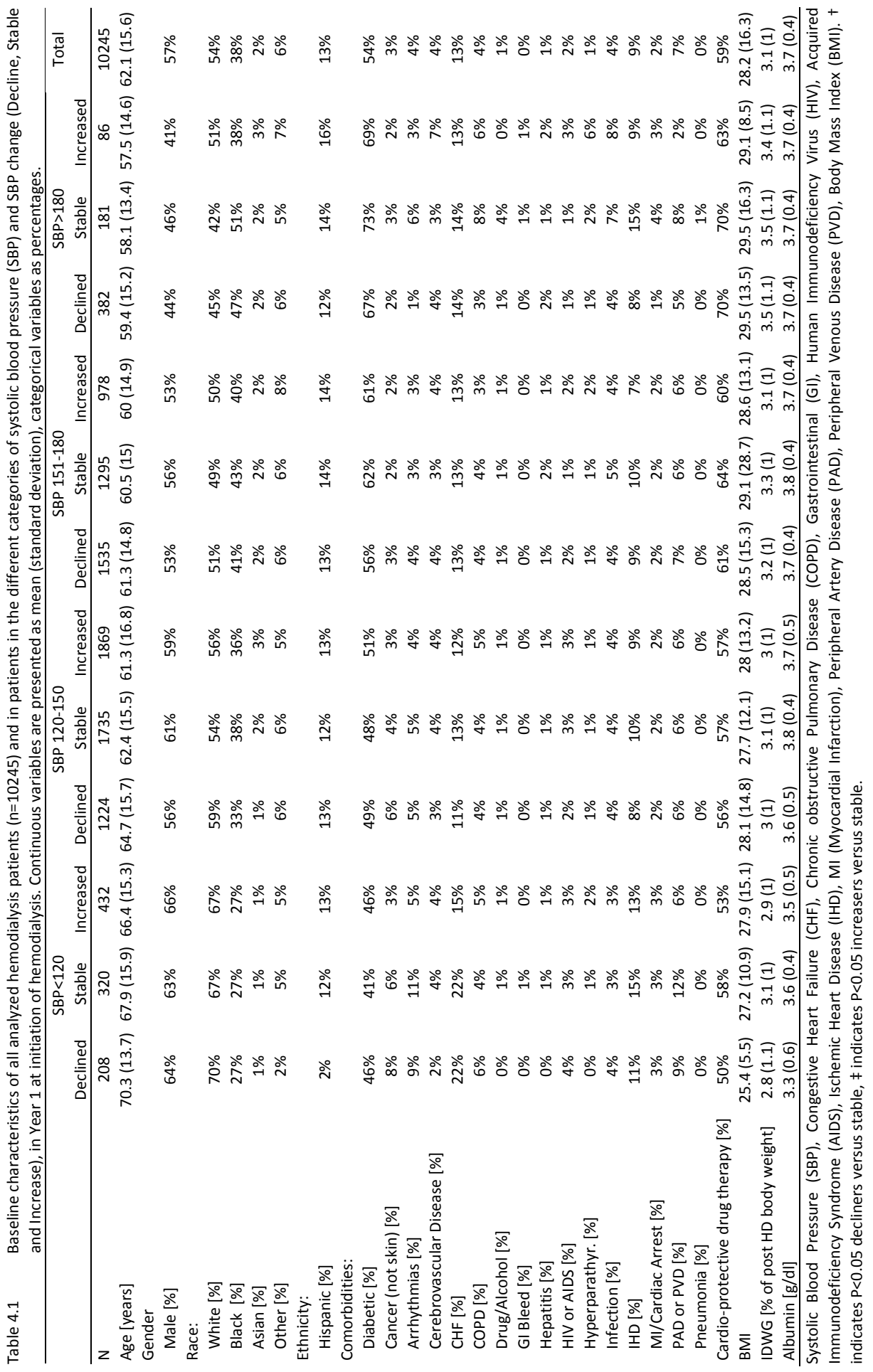




\section{Blood pressure}

Table 4.2 shows SBP and DBP during the first 30 days and the respective slopes of patients with stable, declining and increasing SBP after initiation of dialysis in all patients (see Figure 4.2 for the distribution of SBP in the studied cohort). With CPD prescription, both declines and increases in SBP were attenuated (Figure 4.3) and multivariable LR with SBP slope as the dependent variable showed that use of CPD had impact on the slope in patients with increasing (Beta -0.06, p<0.01) and decreasing (Beta $0.03, p<0.01$ ) slope (data not shown). Stratification of patients in categories of 1 . stable SBP and large 2 . increase $(>5 \mathrm{mmHg}$ per month) and 3 . decrease (<-5 $\mathrm{mmHg}$ per month), showed that patients with stable SBP were more likely to be on CPD (Table 4.3) and thus confirmed the initial findings. Effects of CPD on DBP were similar but less pronounced.

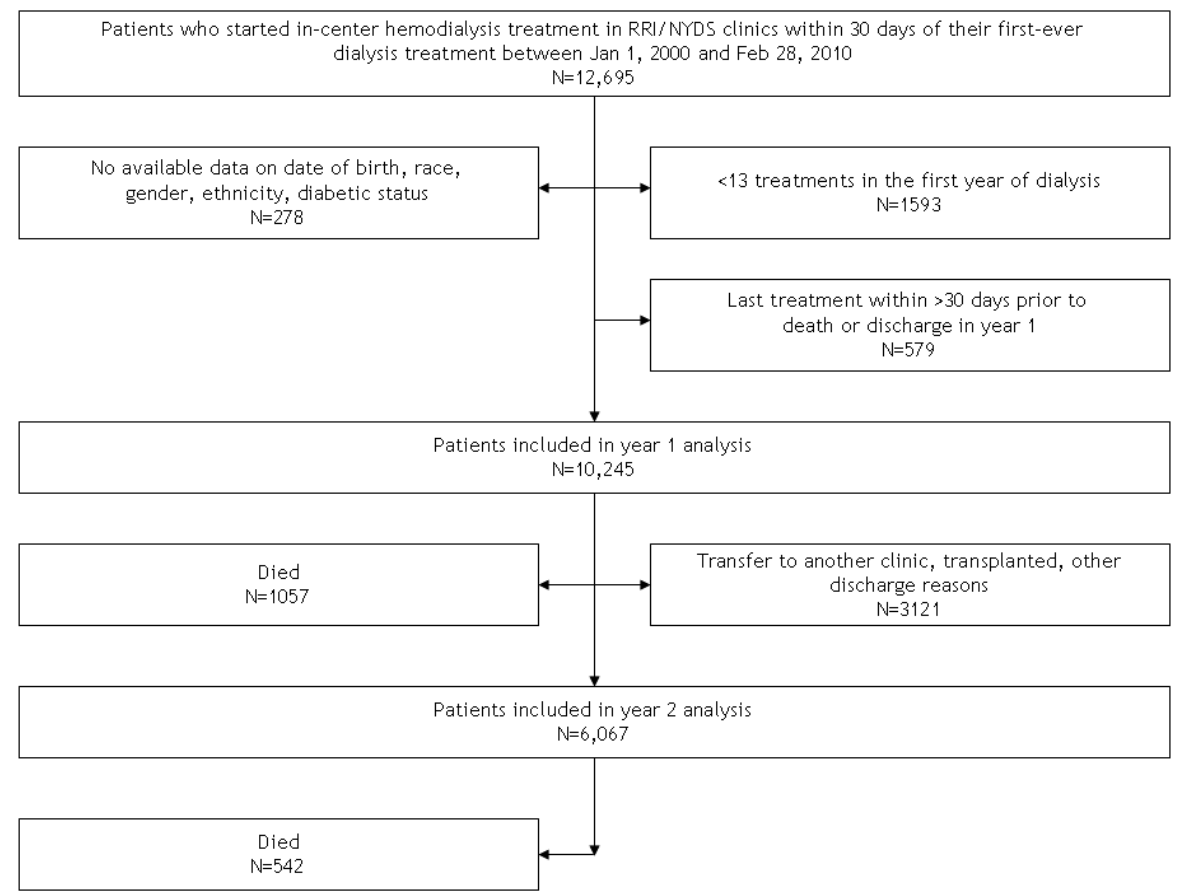

Figure 4.1 Study flowchart. 


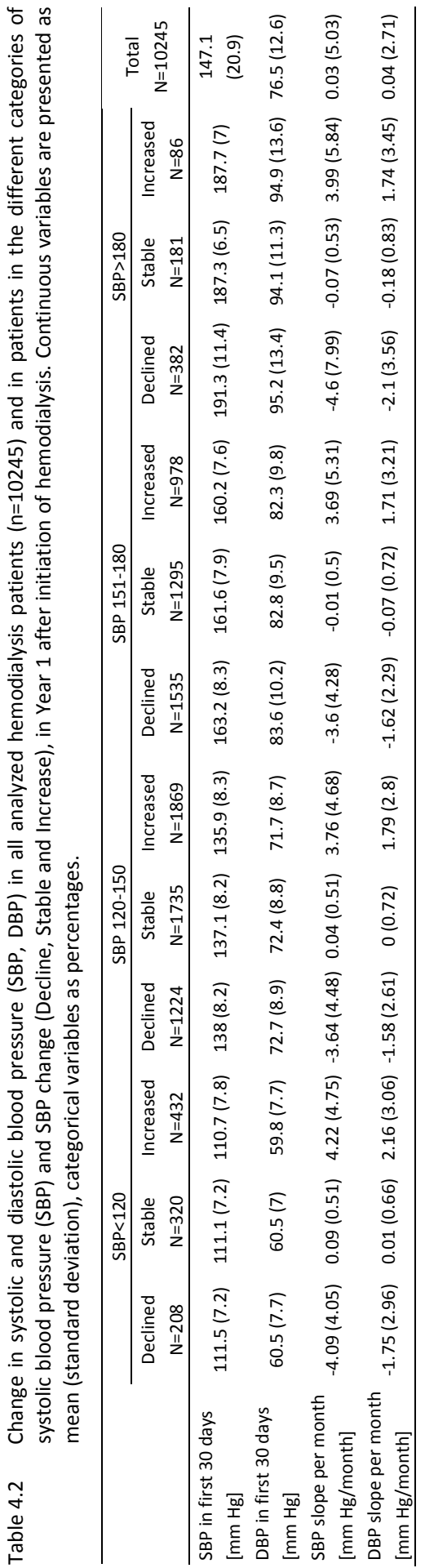




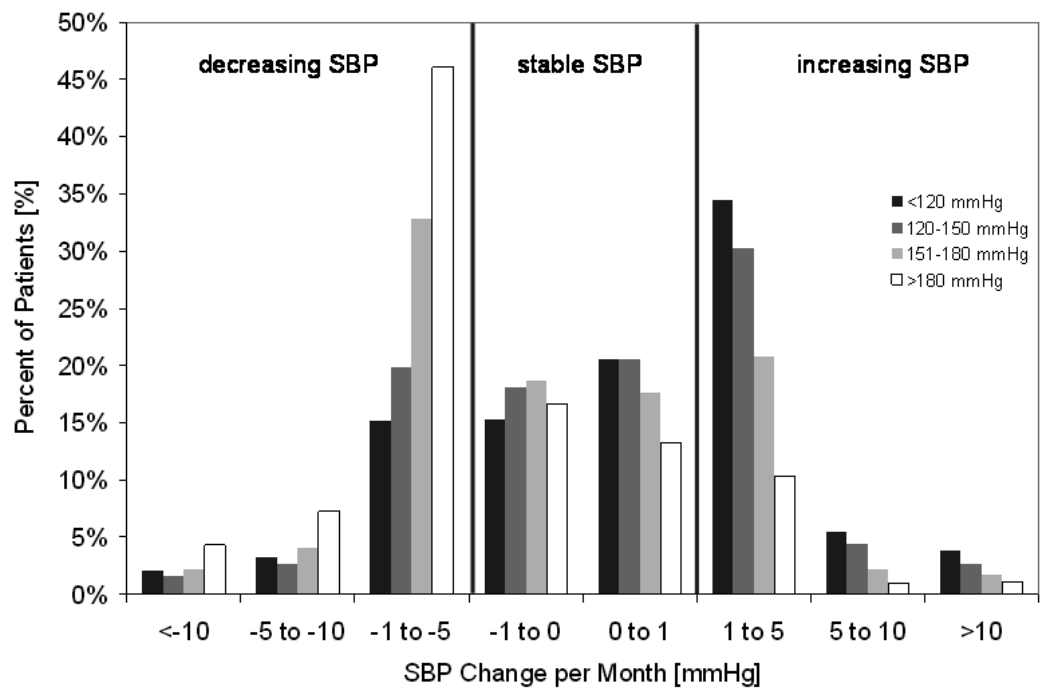

Figure 4.2 Histogram of change of systolic blood pressure (SBP) change per month of patients in all ranges of SBP.

Table 4.3 Change of Systolic Blood Pressure (SBP) in patients with active prescription of cardioprotective drugs (CPD) and without, after stratification in patients with 1 . stable SBP and large 2. increase ( $>5 \mathrm{mmHg}$ per month) and 3 . decrease (<-5 $\mathrm{mmHg}$ per month) of SBP.

\begin{tabular}{lcc}
\hline SBP (Change per Month) & Not on CPD & On CPD \\
\hline Declined $(<-5)$ & $13.8 \%$ & $9.8 \%$ \\
Stable $(-1$ to 1$)$ & $72.0 \%$ & $79.9 \%$ \\
Increased $(>5)$ & $14.1 \%$ & $10.3 \%$ \\
\hline
\end{tabular}



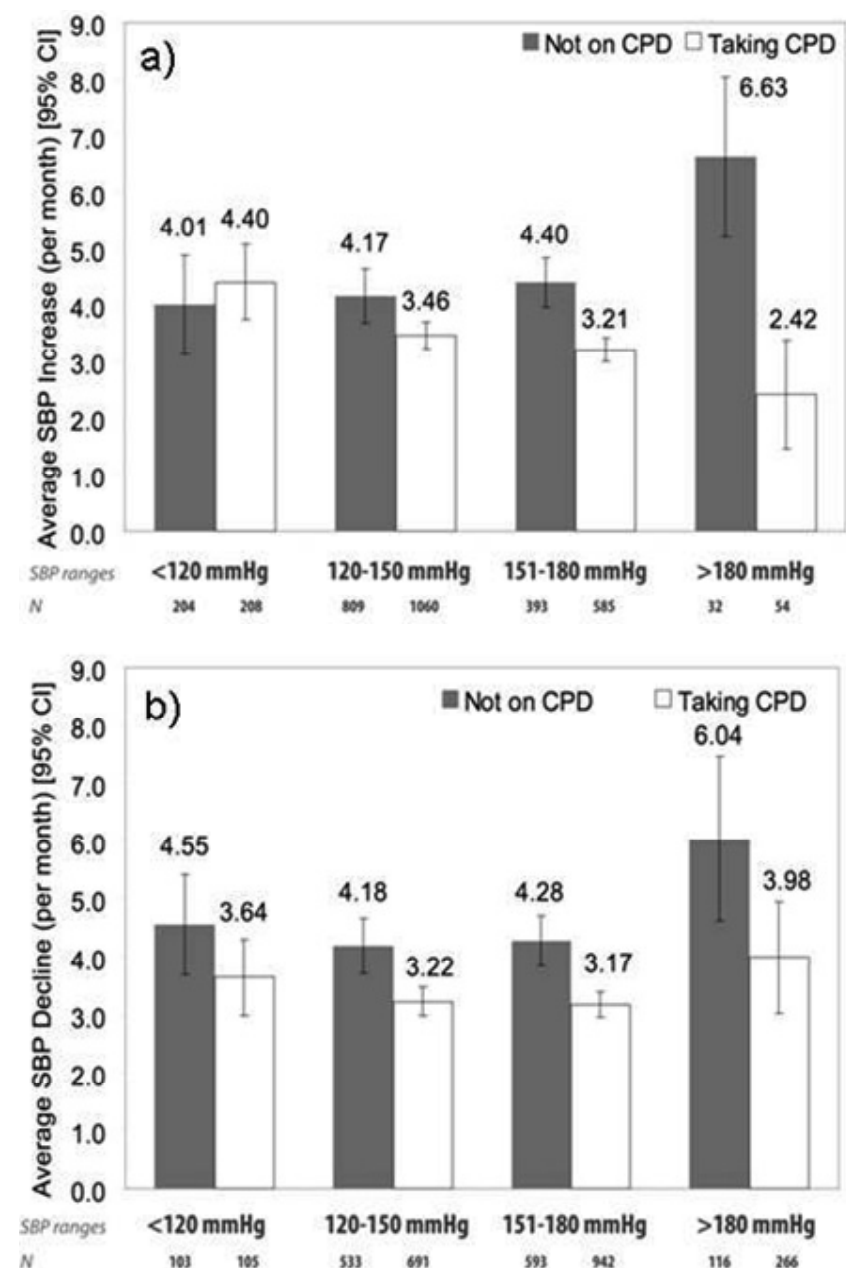

Figure 4.3 Cardioprotective drugs (CPD) and a) increases and b) declines of systolic blood pressure (SBP).

\section{Survival analysis}

Number of deaths and mortality rates per 1000 patient-years after initiation of HD differed between the groups (Table 4.4). Cox Proportional Hazard analysis (Table 4.5) showed that patients with a SBP below $120 \mathrm{mmHg}$ had the poorest, and patients with SBP in the range of 151 to 180 the best survival (Figure 4.4). The latter group served as the reference group in the analysis. Survival was highest in patients with stable SBP independent of SBP level. Patients with decreasing SBP had the poorest survival, 
which was most pronounced in the lowest SBP range. Survival was also poor in patients with SBP increase (Figure 4.4). Similar findings were obtained in the analysis of DBP (Figure 4.5). CPD had a favorable impact on survival (Table 4.5).

Table 4.4 Number of deaths and mortality rates in all patients $(n=10245)$ and in patients in the different categories of systolic blood pressure (SBP) and SBP change (Decline, Stable and Increase).

\begin{tabular}{|c|c|c|c|c|}
\hline & SBP change & $\begin{array}{l}\text { Deaths } \\
\text { [count] }\end{array}$ & $\begin{array}{l}\text { Exposure Time } \\
\text { [days] }\end{array}$ & $\begin{array}{c}\text { Mortality rate }[\mathrm{n} / 1000 \\
\text { patient years] }\end{array}$ \\
\hline \multirow[t]{4}{*}{$\mathrm{SBP}<120 \mathrm{mmHg}$} & Decline $(N=208)$ & 94 & 39011 & 880.10 \\
\hline & Stable $(\mathrm{N}=320)$ & 28 & 99421 & 102.87 \\
\hline & Increase $(\mathrm{N}=432)$ & 79 & 104945 & 274.95 \\
\hline & All Patients $(\mathrm{N}=960)$ & 201 & 243377 & 301.65 \\
\hline \multirow[t]{4}{*}{ SBP $120-150 \mathrm{mmHg}$} & Decline ( $N=1224)$ & 226 & 316199 & 261.06 \\
\hline & Stable $(\mathrm{N}=1735)$ & 86 & 569950 & 55.11 \\
\hline & Increase $(\mathrm{N}=1869)$ & 202 & 505238 & 146.03 \\
\hline & All Patients $(\mathrm{N}=4828)$ & 514 & 1391387 & 134.93 \\
\hline \multirow[t]{4}{*}{ SBP $151-180 \mathrm{mmHg}$} & Decline ( $N=1535)$ & 159 & 433736 & 133.89 \\
\hline & Stable $(\mathrm{N}=1295)$ & 47 & 427963 & 40.11 \\
\hline & Increase $(\mathrm{N}=978)$ & 76 & 261933 & 105.98 \\
\hline & All Patients ( $\mathrm{N}=3808)$ & 282 & 1123632 & 91.67 \\
\hline \multirow[t]{4}{*}{$\mathrm{SBP}>180 \mathrm{mmHg}$} & Decline ( $N=382$ ) & 46 & 112594 & 149.22 \\
\hline & Stable $(\mathrm{N}=181)$ & 7 & 60411 & 42.32 \\
\hline & Increase $(\mathrm{N}=86)$ & 7 & 22184 & 115.25 \\
\hline & All Patients ( $\mathrm{N}=649)$ & 60 & 195189 & 112.28 \\
\hline All Patients $(\mathrm{N}=10245)$ & & 1057 & 2953585 & 130.71 \\
\hline
\end{tabular}

\section{Sensitivity analyses}

For the first sensitivity analysis only data of patients with slopes showing P-values below 0.05 , were included $(n=8395)$. The result of this survival analysis was consistent with the original analyses, thus the analysis did not appear to be confounded by the certainty of the slope computation.

For the second sensitivity analysis, first and last six SBP measurements were excluded, and the results were again consistent with the original analyses. Slope computation did not appear to be confounded by outliers in the beginning or the end of the observation period.

For the third sensitivity analysis patients with the largest changes in SBP were excluded. The relation of changes in SBP and mortality remained the same in this analysis.

The fourth sensitivity analysis defining patients with stable, increasing and decreasing SBP according to the upper and lower, respectively, limits of their $95 \%$ confidence interval limit, showed similar results as the primary analysis. 
Table 4.5 Cox Proportional Hazards model for Survival Analysis in Year 1.

\begin{tabular}{|c|c|c|}
\hline & Hazard Ratio $(95 \% \mathrm{Cl})$ & P-value \\
\hline SBP $<120 \mathrm{mmHg}$, Declined & 8.375 (5.805 to 12.084$)$ & $<0.0001$ \\
\hline SBP $<120 \mathrm{mmHg}$, Stable & $1.344(0.835$ to 2.164$)$ & 0.223 \\
\hline $\mathrm{SBP}<120 \mathrm{mmHg}$, Increased & 3.594 (2.483 to 5.202$)$ & $<0.0001$ \\
\hline SBP 120 to $150 \mathrm{mmHg}$, Declined & $3.731(2.700$ to 5.156$)$ & $<0.0001$ \\
\hline SBP 120 to $150 \mathrm{mmHg}$, Stable & $1.099(0.766$ to 1.578$)$ & 0.607 \\
\hline SBP 120 to $150 \mathrm{mmHg}$, Increased & 2.678 (1.937 to 3.702$)$ & $<0.0001$ \\
\hline SBP 151 to $180 \mathrm{mmHg}$, Declined & 2.634 (1.893 to 3.665$)$ & $<0.0001$ \\
\hline SBP 151 to $180 \mathrm{mmHg}$, Stable & Reference Group & \\
\hline SBP 151 to $180 \mathrm{mmHg}$, Increased & 2.516 (1.743 to 3.632$)$ & $<0.0001$ \\
\hline SBP $>180 \mathrm{mmHg}$, Declined & $3.344(2.213$ to 5.053$)$ & $<0.0001$ \\
\hline SBP $>180 \mathrm{mmHg}$, Stable & 1.125 (0.507 to 2.497$)$ & 0.773 \\
\hline SBP $>180 \mathrm{mmHg}$, Increased & 2.995 (1.349 to 6.650$)$ & 0.007 \\
\hline Age [years] & $1.028(1.023$ to 1.033$)$ & $<0.0001$ \\
\hline Male [yes/no] & $1.146(1.009$ to 1.301$)$ & 0.036 \\
\hline Race=White [yes/no] & 1.260 (1.090 to 1.458$)$ & 0.002 \\
\hline Ethnicity=Hispanic [yes/no] & $0.713(0.564$ to 0.900$)$ & 0.004 \\
\hline COPD [yes/no] & $0.576(0.391$ to 0.848$)$ & 0.005 \\
\hline Drug/Alcohol [yes/no] & 0.552 (0.244 to 1.249$)$ & 0.154 \\
\hline Hepatitis [yes/no] & $1.970(0.812$ to 4.778$)$ & 0.134 \\
\hline Hyperparathyroidism [yes/no] & $0.588(0.277$ to 1.246$)$ & 0.166 \\
\hline Infection [yes/no] & $0.456(0.262$ to 0.796$)$ & 0.006 \\
\hline CPD therapy [yes/no] & $0.717(0.630$ to 0.815$)$ & $<0.0001$ \\
\hline Albumin $[\mathrm{g} / \mathrm{dL}]$ & $0.162(0.145$ to 0.181$)$ & $<0.0001$ \\
\hline IDWG [\%post HD body weight] & $0.926(0.866$ to 0.990$)$ & 0.023 \\
\hline
\end{tabular}

Chronic Obstructive Pulmonary Disease (COPD), cardioprotective drug (CPD), Interdialytic weight gain (IDWG). Covariables (not related to blood pressure) with P-value in the Model larger than 0.2 are not shown (Asian race, Diabetes, pre-existing anemia, cancer, arrhythmias, cerebrovascular disease, Congestive Heart Failure, Gastrointestinal Bleed, Human Immunodeficiency Virus or Acquired Immunodeficiency Syndrome, Ischemic Heart Disease, Myocardial Infarction or Cardiac Arrest, Peripheral arterial or venous disease, Pneumonia and Body mass index. 


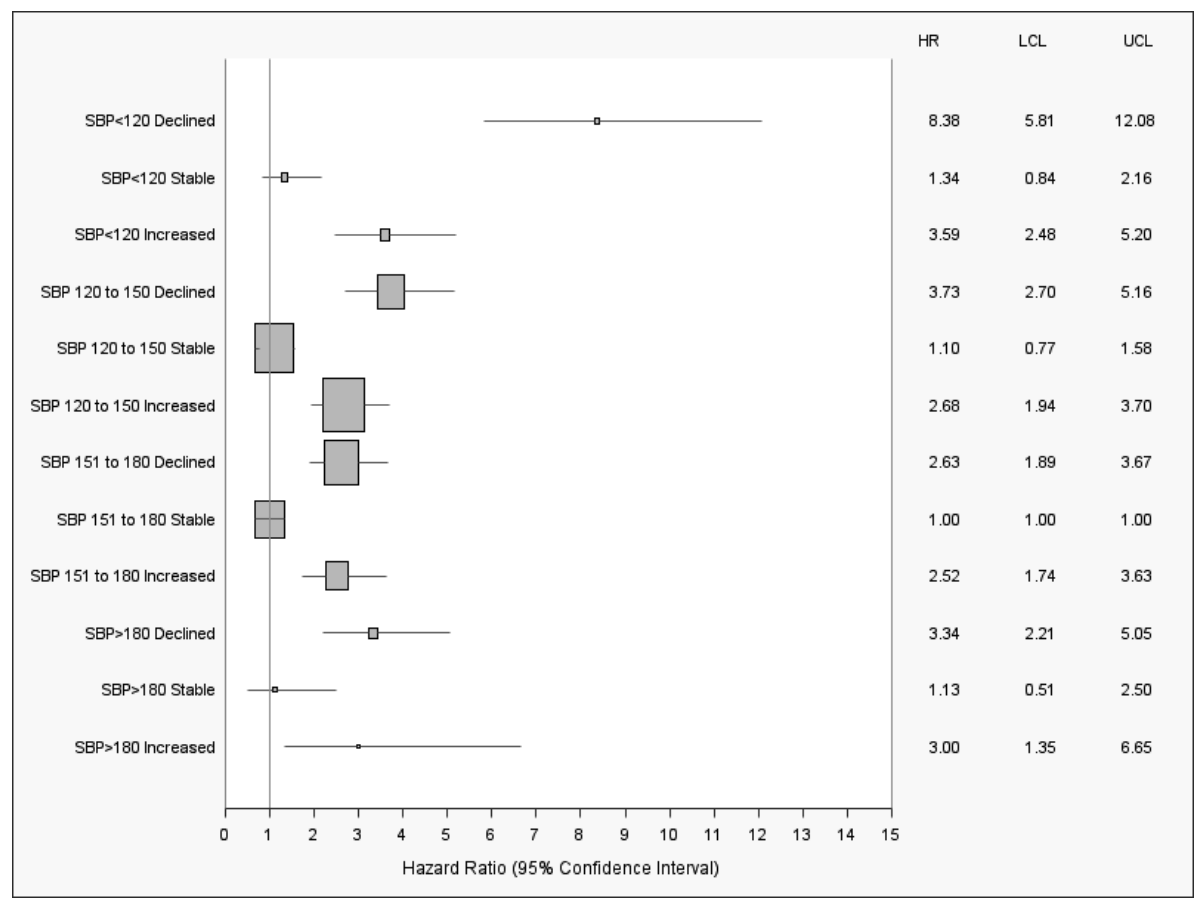

Figure 4.4 Levels of systolic blood pressure (SBP) and temporal changes (reflected by changes in slope) and its relation to Hazard Ratio for Death in all 12 groups. A P-value below 0.05 is indicated with an asterisk.

\section{Validation of survival analysis}

In the subset validation analysis (Figure 4.6) changes in SBP in patients with CPD therapy and those when CPD was not being prescribed, were consistent with the main analysis. The positive effect on patients' survival of the quality initiative is also illustrated in a Kaplan Meier survival analysis (Figure 4.7). Repetition of survival analysis with seasonality as an adjustment factor showed similar results like the main analysis (Figure 4.8). 6067 (at this point prevalent) HD patients were included in the analysis in Year 2. Results of survival analysis according to SBP categories and temporal changes in Year 2 (Figure 4.9) were consistent with the results in Year 1 (Figure 4.4). Repetition of the analysis of the relation of temporal changes of SBP at all levels of SBP to hazard ratio using cardiovascular mortality as outcome only showed similar findings as compared to the primary analysis (Figure 4.10). 


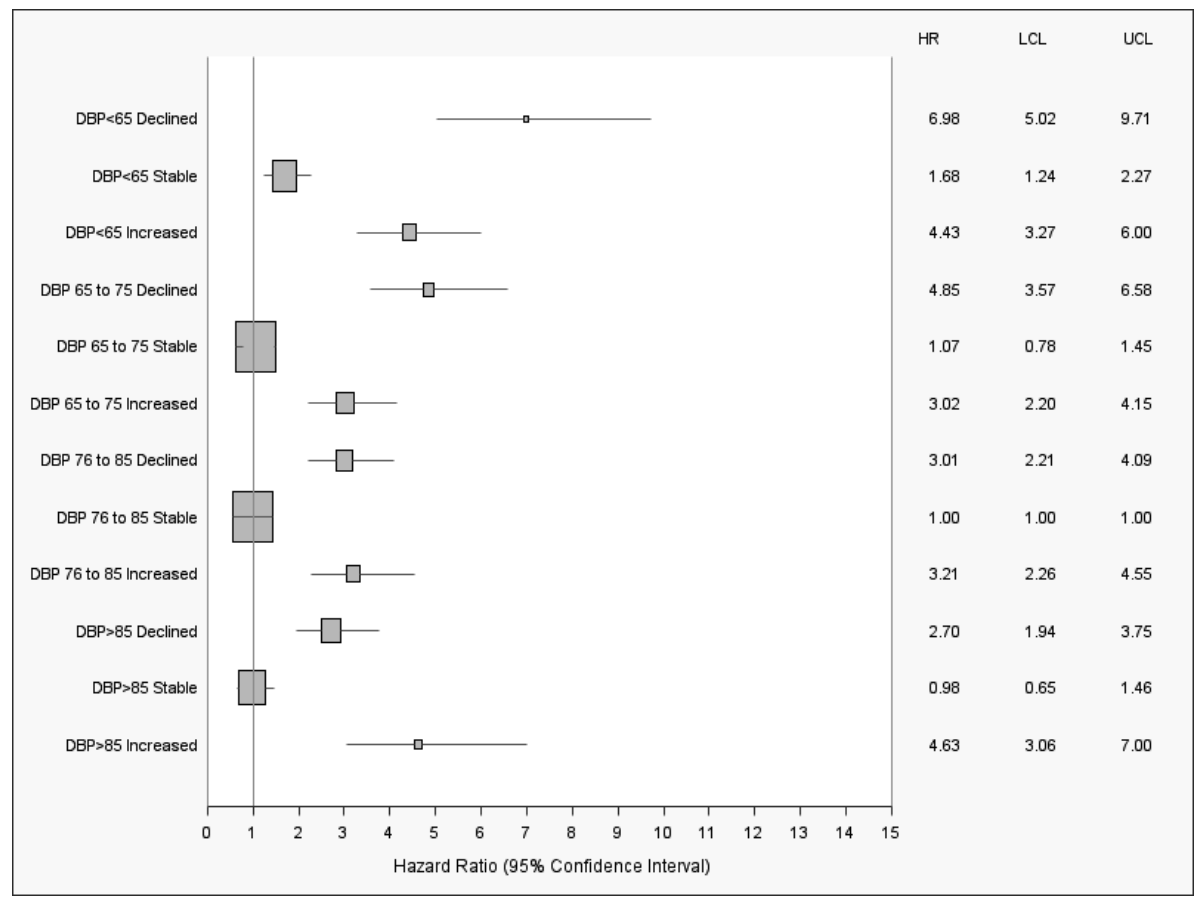

Figure 4.5 Levels of diastolic blood pressure (DBP) and temporal changes (reflected by changes in slope) and its relation to Hazard Ratio for Death in all 12 analyzed groups. A P-value below 0.05 is indicated with an asterisk. 

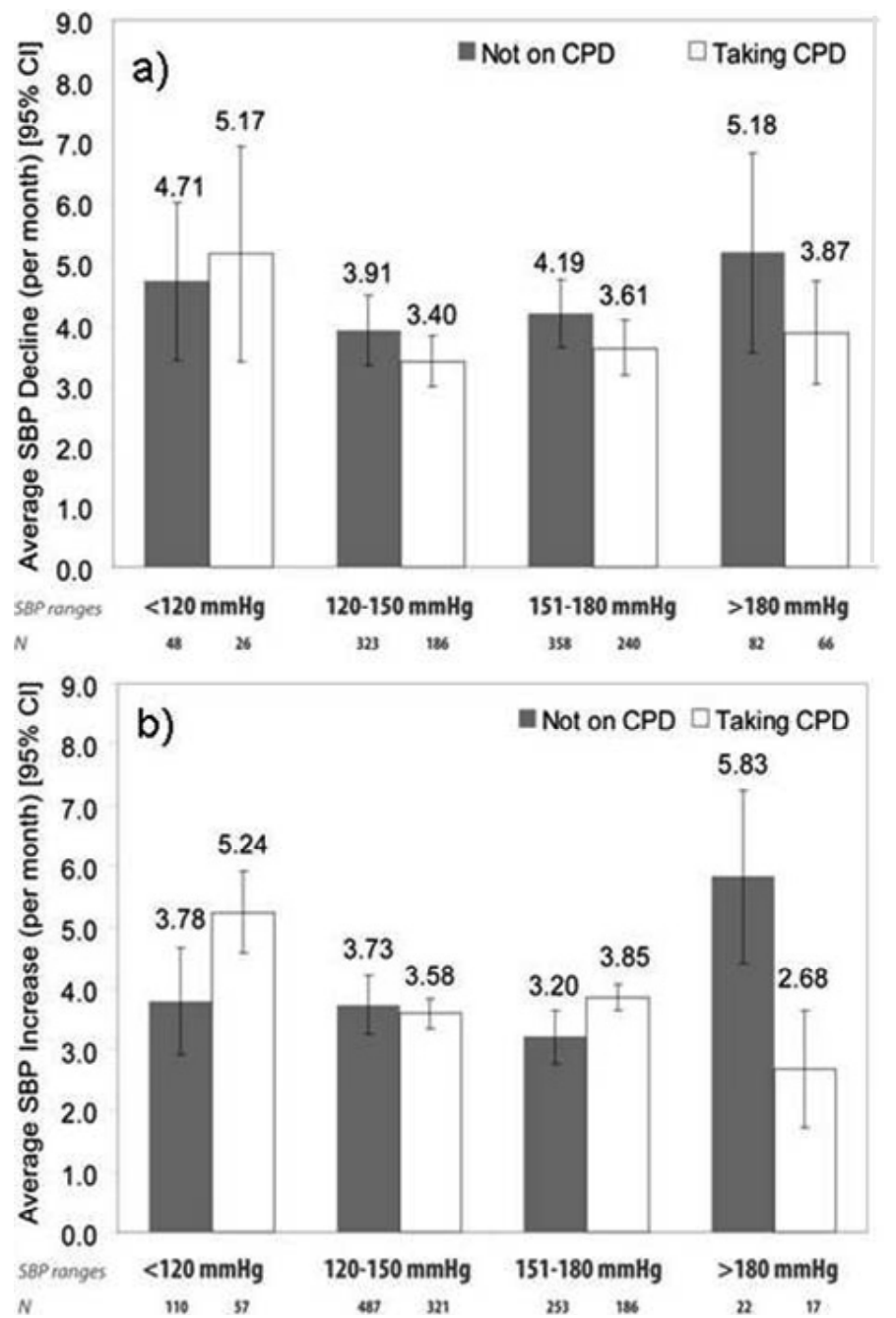

Figure 4.6 Subset validation analysis to validate the relation of therapy with cardioprotective drugs (CPD) and declines and increases in systolic blood pressure (SBP). Patients included in the subset were patient initiating dialysis prior to 2006 and were not on CPD and patients who started dialysis, after a CPD quality initiative in 2007, and having an active CPD prescription. 


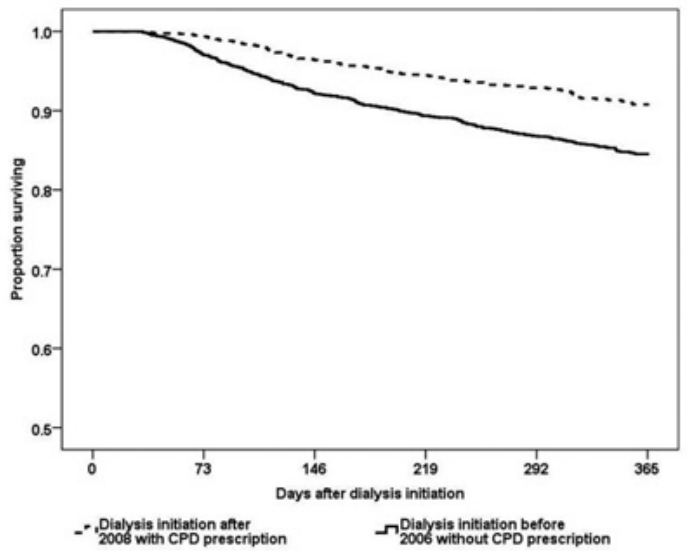

Figure 4.7 Kaplan Meier Survival curves in two subsets of patients which 1) initiated hemodialysis prior to 2006 and were not on cardioprotective drugs (CPD), and 2) patients who started hemodialysis after a quality initiative (suggesting use of CPD in all patients regardless of blood pressure) in 2008 and were on CPD.

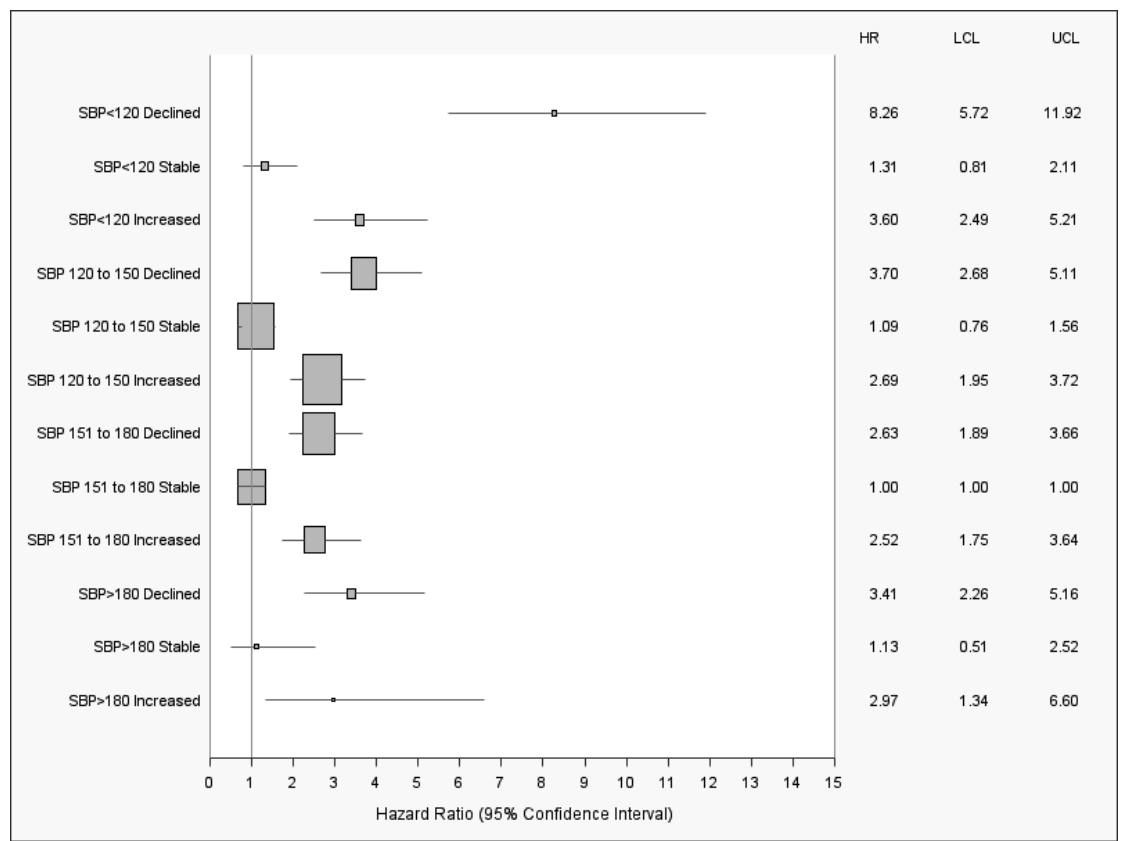

Figure 4.8 Levels of systolic blood pressure (SBP) and temporal changes (reflected by changes in slope) and its relation to Hazard Ratio for Death in all 12 groups after adjustment for season of death or season for end of year 1 in survivors (Spring, Summer, Autumn and Winter). A P-value below 0.05 is indicated with an asterisk. 


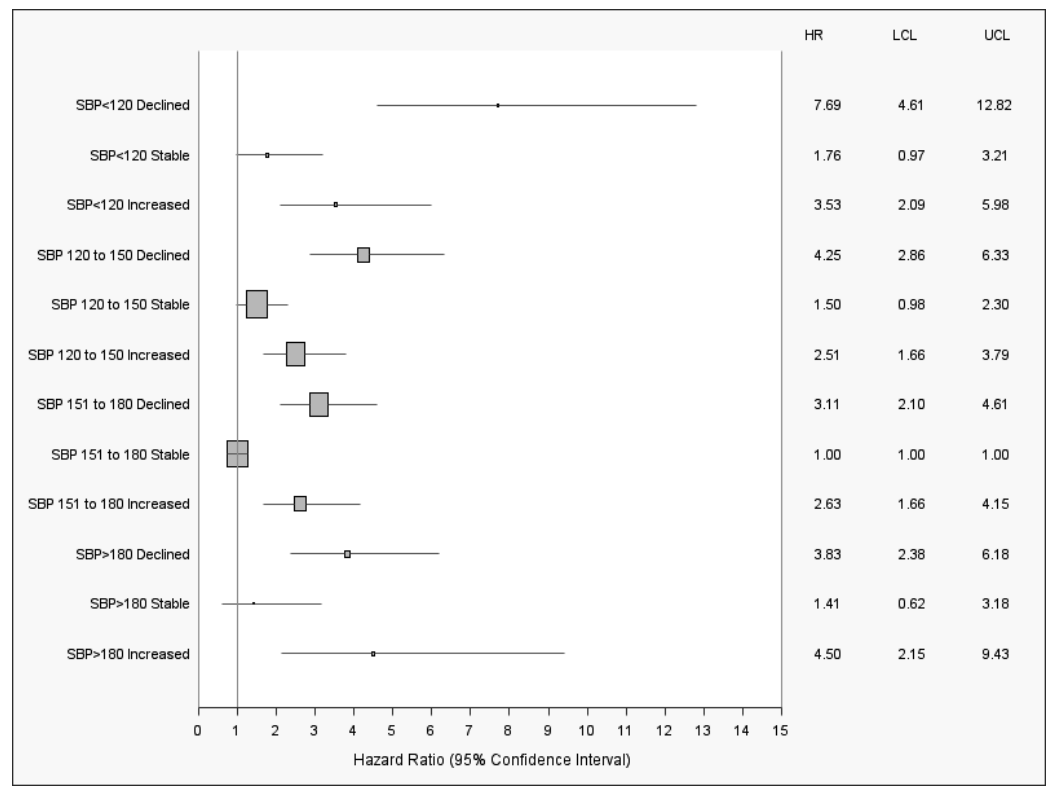

Figure 4.9 Validation of the analysis on levels of systolic blood pressure (SBP) and temporal changes (reflected by changes in slope) in Year 2 and its relation to Hazard Ratio for Death in all 12 groups. A P-value below 0.05 is indicated with an asterisk.

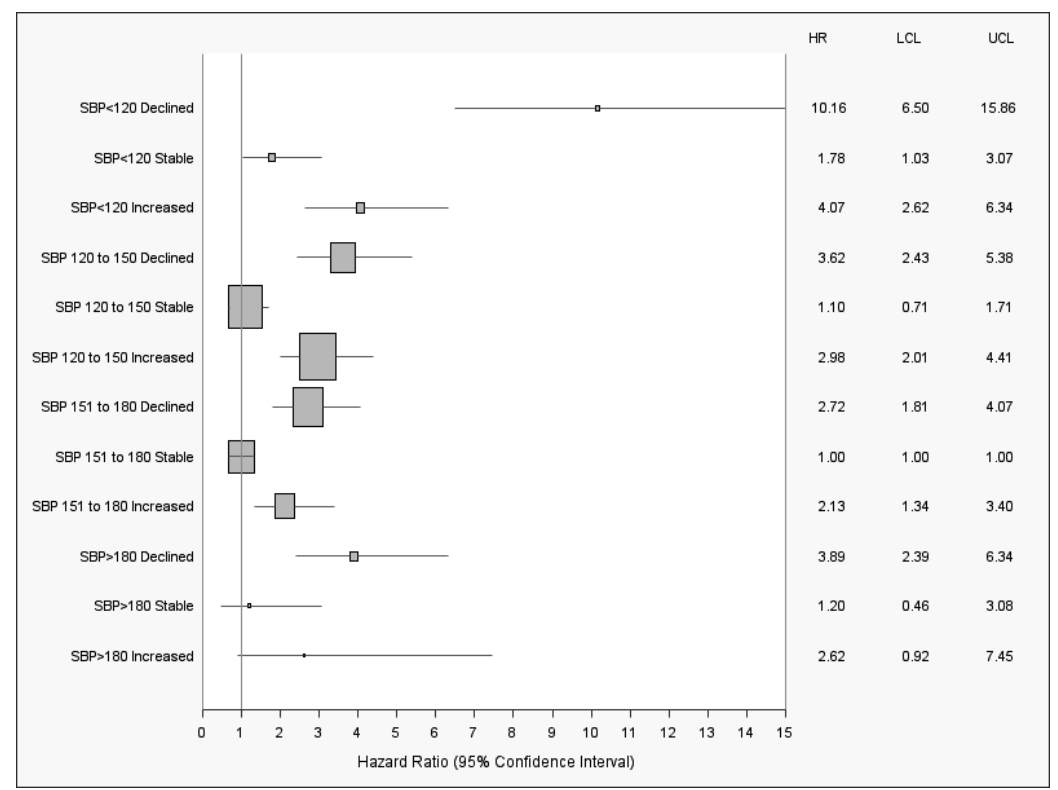

Figure 4.10 Levels of systolic blood pressure (SBP) and temporal changes (reflected by changes in slope) and its relation to Hazard Ratio for cardiovascular death in all 12 analyzed groups. A P-value below 0.05 is indicated with an asterisk. 


\section{Discussion}

To the best of our knowledge this is the first observational cohort study in a large group of HD patients evaluating over one year the association of absolute SBP and DBP levels and subsequent temporal BP changes on mortality. The main finding is that mortality in the first year on HD was greater in patients with both, declining or increasing BP compared to stable BP, independent of BP levels (Figure 4.4, Figure 4.5); this result was corroborated in Year 2 (compare Figure 4.4 and Figure 4.9).

Temporal changes in SBP and DBP rather than the BP category, had greater impact on survival (Figure 4.4). Furthermore our findings suggest a potential beneficial effect of using CPDs in potentially stabilizing BP changes over time and improving survival.

The explanation of these findings are two different operative vectors: One, the declining BP due to failure of ventricular function and thus marked increase in mortality, and the other is increased BP, for reasons which remain to be determined exactly, but likely include salt and fluid overload, with lesser effect on mortality than in those with declining blood pressure. In addition it is remarkable that many comorbidities and laboratory parameters lost significance in the Cox Proportional Hazard Model, after inclusion of temporal changes of SBP and also CPD. It is well known that cardiovascular disease is the leading cause of death, but it is remarkable to what extent temporal changes of SBP appear to influence survival in this analysis (Table 4.5, Figure 4.4).

The favorable effect of CPD in improving mortality both in patients with declining and increasing in BP is unexpected, although it appears concordant with reports of cardioprotective effects of CPD by the results in few performed long-term RCTs in HD patients. Extensive meta-analyses showed that reduced mortality risk was observed in patients on CPD without substantial BP change ${ }^{20,21}$. In this analysis calcium channel blockers have not been included, although a mortality reduction has been reported by its use ${ }^{22}$. However, due to the lack of data from RCTs on the cardioprotective effect of this drug class in dialysis patients, we decided to not include this drug class in our analysis.

Relative to other BP categories, CPD use is associated with a smaller decrease in BP in patients with baseline levels of below $120 \mathrm{mmHg}$. This could be the result of medication effects counteracting the adverse consequences of progressive cardiomyopathy. In patients with increasing BP the reduction in BP by CPD appears compatible with antihypertensive effects of the drugs. 
All Year 2 analyses of survival were consistent with Year 1 (compare Figure 4.4 and Figure 4.9). This confirmatory test of our basic argument also affirms the continued use of CPD in all patients at risk. This is also corroborated by the effect seen by the quality initiative (Figure 4.7).

A major strength of this analysis is the large sample size and the balanced distribution of patients in all analyzed groups (Table 4.1). Patients of 51 different dialysis clinics in different states and geographical regions were included in this observational cohort study. Widespread patient population and the considerably large sample size, the study cohort may be assumed generalizable to the HD population in the United States. Selection of incident patients allowed the study of the evolution of BP virtually without effects of HD treatment at study baseline (defined as the first month after the first HD treatment) and after initiation of HD and its effects on the cardiovascular system.

The repetition of the survival analysis in Year $2(n=6067)$ counteracts arguments of survival bias which may be made in analyses in prevalent chronic HD patients. In addition this observational study allowed analysis of a prevalent patient population with equal HD vintage (compare Figure 4.4 and Figure 4.9). Similar relations between cardiovascular mortality and temporal evolution of SBP (Figure 4.10) confirm the reliability of our findings. Repeated analysis with patients in the highest quartiles of change excluded show that the results were not driven by a few patients with large changes of SBP.

The sensitivity analysis of univariable LR to estimate the slope for every individual patient was validated by narrowing the criteria for defining patients as stable, and including only patients whose SBP slope increased and decreased significantly. The latter method confirmed that outlier measurements did not influence the results. The second sensitivity analysis, which excluded the first and last six SBP measurements in the observation period suggests that the findings were not confounded by outliers. The third and the fourth sensitivity analysis showed the statistical validity of our approach and support the hypothesized biological phenomena. Further, survival analyses were repeated with adjustment for season (Spring, Summer, Autumn and Winter) of death or end of year 1 for survivors to exclude confounding by seasonal differences of mortality. Results were identical after adjustment of season (Figure 4.8).

Limitations of the analysis included the absence of information on SBP prior to initiation of HD, which would have been of interest when investigating the temporal evolution subsequent to HD initiation. Lack of knowledge on the degree of body hydration of patients is another limitation, but difficult to overcome with current 
technologies $^{23}$. Knowledge of cardiac function and structure by cardiac magnetic resonance imaging (cMRI) or echocardiography would have been of high value. The use of different BP measurements methods, such as home or ambulatory methods, may have been preferable, but even these methods are to a degree difficult to interpret in a HD population because of the increase in volume and BP which occurs progressively during the interdialytic interval. While the measurements of pre- and postdialysis BP are imprecise ${ }^{24}$, their frequent measurement and the relationship between levels and outcomes provide reassurance. Another consideration may be that the analysis of drugs could be confounded by bias by indication, this can be excluded due to 1 . the large sample size included in this analysis and 2 . The subset validation analysis comparing patients initiating dialysis prior to 2004 as compared to those starting dialysis after 2007 (Figure 4.6 and Figure 4.7). An additional concern is a potential bias induced by varying duration of CPD prescription, since this parameter was not captured in this analysis. Several limitations also exist on the documentation of comorbidities and CPD. For the latter in particular: 1) lack of knowledge on dose and frequency of CPD, 2) documentation drugs depends on the accuracy of the administrative staff, and 3) the possibility of erratic patient adherence, with the methods of analysis used, none of these issues are likely to cause any systematic error or bias (compare Figure 4.3 and Figure 4.6).

An important future analysis remains the relation between BP level, temporal changes of $B P, C P D$ use on survival in patients prior to initiation of dialysis. Another main research question remains: 'To what extent should BP increases and decreases be managed by sodium restriction and ultrafiltration or by use of CPD at different degrees of cardiac damage'. However, this question can only be answered by an adequately powered RCT.

In conclusion, we found that blood pressure readings are best interpreted using trends and should not taken in isolation. The clinical problem posed by these data is that an apparently normal BP may be misinterpreted as being satisfactory. If absolute $\mathrm{BP}$ values are in the normal range due to cardiac decompensation, observation of $\mathrm{BP}$ values only may provide a false sense of security when a declining particularly or increasing trend is not appreciated. Evaluation of cardiac and fluid status is essential in interpreting blood pressure levels. The favorable effect observed with the use of $\mathrm{CPD}$ and the mechanism of action requires further evaluation in prospective clinical trials. 


\section{References}

1. Vasan RS, Larson MG, Leip EP, Evans JC, et al. Impact of high-normal blood pressure on the risk of cardiovascular disease. N Engl J Med. 2001;345:1291-7.

2. Lewington S, Clarke R, Qizilbash N, Peto R, et al. Age-specific relevance of usual blood pressure to vascular mortality: a meta-analysis of individual data for one million adults in 61 prospective studies. Lancet. 2002;360:1903-13.

3. Agarwal R, Nissenson AR, Batlle D, Coyne DW, et al. Prevalence, treatment, and control of hypertension in chronic hemodialysis patients in the United States. Am J Med. 2003;115:291-7.

4. Rocco MV, Yan G, Heyka RJ, Benz R, et al. Risk factors for hypertension in chronic hemodialysis patients: baseline data from the HEMO study. Am J Nephrol. 2001;21:280-8.

5. Levin NW, Kotanko P, Eckardt KU, Kasiske BL, et al. Blood pressure in chronic kidney disease stage 5Dreport from a Kidney Disease: Improving Global Outcomes controversies conference. Kidney Int. 2009;77:273-84.

6. Clinical practice guidelines for hemodialysis adequacy, update 2006. Am J Kidney Dis. 2006;48 Suppl 1:S2-90.

7. Fletcher AE, Beevers DG, Bulpitt CJ, Butler A, et al. The relationship between a low treated blood pressure and IHD mortality: a report from the DHSS Hypertension Care Computing Project (DHCCP). J Hum Hypertens. 1988;2:11-5.

8. Waller PC, Isles CG, Lever AF, Murray GD, et al. Does therapeutic reduction of diastolic blood pressure cause death from coronary heart disease? J Hum Hypertens. 1988;2:7-10.

9. Protogerou $A D$, Safar ME, laria $P$, Safar $H$, et al. Diastolic blood pressure and mortality in the elderly with cardiovascular disease. Hypertension. 2007;50:172-80.

10. Messerli FH, Mancia G, Conti CR, Hewkin AC, et al. Dogma disputed: can aggressively lowering blood pressure in hypertensive patients with coronary artery disease be dangerous? Ann Intern Med. 2006; 144:884-93.

11. Samuelsson O, Wilhelmsen L, Andersson OK, Pennert K, et al. Cardiovascular morbidity in relation to change in blood pressure and serum cholesterol levels in treated hypertension. Results from the primary prevention trial in Goteborg, Sweden. JAMA. 1987;258:1768-76.

12. Alderman $\mathrm{MH}$, Ooi $\mathrm{WL}$, Madhavan S, Cohen $\mathrm{H}$. Treatment-induced blood pressure reduction and the risk of myocardial infarction. JAMA. 1989;262:920-4.

13. Cooper SP, Hardy RJ, Labarthe DR, Hawkins CM, et al. The relation between degree of blood pressure reduction and mortality among hypertensives in the Hypertension Detection and Follow-Up Program. Am J Epidemiol. 1988;127:387-403.

14. Cruickshank JM, Thorp JM, Zacharias FJ. Benefits and potential harm of lowering high blood pressure. Lancet. 1987;1:581-4.

15. Bangalore S, Messerli FH, Wun CC, Zuckerman AL, et al. J-curve revisited: an analysis of blood pressure and cardiovascular events in the Treating to New Targets (TNT) trial. Eur Heart J. 2010;31:2897-908.

16. Li Z, Lacson E, Jr, Lowrie EG, Ofsthun NJ, et al. The epidemiology of systolic blood pressure and death risk in hemodialysis patients. Am J Kidney Dis. 2006;48:606-15.

17. Myers OB, Adams C, Rohrscheib MR, Servilla KS, et al. Age, race, diabetes, blood pressure, and mortality among hemodialysis patients. J Am Soc Nephrol. 2010;21:1970-8.

18. Zager PG, Nikolic J, Brown RH, Campbell MA, et al. "U" curve association of blood pressure and mortality in hemodialysis patients. Medical Directors of Dialysis Clinic, Inc. Kidney Int. 1998;54:561-9.

19. Centers for Disease Control and Prevention (U.S.), Centers for Medicare \& Medicaid Services (U.S.): ICD-9-CM international classification of diseases, ninth revision, clinical modification, sixth edition. In, Official ed, U.S. Dept. of Health and Human Services and the Centers for Medicare and Medicaid Services, 2008, pp 1 CD-ROM

20. Agarwal R, Sinha AD. Cardiovascular protection with antihypertensive drugs in dialysis patients. Systematic review and meta-analysis. Hypertension. 2009;53:860-6. 
21. Heerspink HJ, Ninomiya T, Zoungas S, de Zeeuw D, et al. Effect of lowering blood pressure on cardiovascular events and mortality in patients on dialysis: a systematic review and meta-analysis of randomised controlled trials. Lancet. 2009;373:1009-15.

22. Tepel M, Giet MV, Park A, Zidek W. Association of calcium channel blockers and mortality in haemodialysis patients. Clin Sci (Lond) 2002;103:511-5.

23. Raimann J., Li L., Tyagi S., Levin NW., et al. A fresh look at dry weight. Hemodialysis International. 2008;12:395-405.

24. Agarwal R, Peixoto AJ, Santos SF, Zoccali C. Pre- and postdialysis blood pressures are imprecise estimates of interdialytic ambulatory blood pressure. Clin J Am Soc Nephrol. 2006;1:389-98. 


\section{Chapter 5}

Relation between trends in body temperature and outcome in incident hemodialysis patients

Len A Usvyat, Jochen G Raimann, Mary Carter, Frank M van der Sande, Jeroen P Kooman, Peter Kotanko, Nathan W. Levin

Nephrol Dial Transplant. 2012;27:3255-3263 


\section{Abstract}

Various biochemical and physiological variables are related to outcome in hemodialysis (HD) patients. However, the prognostic implications of trends in body temperature (BT) in this population have not yet been studied. The aim of this study was to assess the relationship between trends in BT and outcome in incident HD patients.

6742 incident HD patients without thyroid disease from the Renal Research Institute were followed for one year. Patients were divided into tertiles of initial pre-dialysis BT (tertile $1:<=36.47^{\circ} \mathrm{C}$, tertile $2:>36.47$ to $36.71^{\circ} \mathrm{C}$, tertile $3:>36.7^{\circ} \mathrm{C}$ ) and further classified according to the change in $\mathrm{BT}$ (increased: $>0.01^{\circ} \mathrm{C} / \mathrm{month}$, decreased: $<-0.01^{\circ} \mathrm{C} /$ month, and stable, with change between -0.01 and $+0.01^{\circ} \mathrm{C} /$ month) during the first year of treatment. The reference group is tertile 2 of initial temperature with stable BT. Cox regression was used for survival analyses. Analyses were repeated for patients who survived the first year and were treated for $\geq 1$ month in Year 2 .

BT decreased in 2903 patients, remained stable in 2238 patients and increased in 1601 patients. After adjustment for multiple risk factors, hazard ratios (HR) for mortality were higher for those groups in whom, irrespective of the initial BT, BT increased or declined, as compared to the reference group during follow-up [HR between 1.46 and 2.27]. The best survival was observed in the group with the highest BT at baseline and stable BT during the follow-up period [HR 0.50]. 


\section{Introduction}

Mortality in hemodialysis (HD) patients is high. Many prognostic factors have been identified in this patient population, most of them related to cardiovascular disease, inflammation, or malnutrition. Other physiological variables, such as blood pressure, have also found to be related to outcome in HD patients. However, this relationship appears to be complex, with both low and high blood pressures related to poorer outcome $^{1}$.

One important physiological variable which has received no attention so far as a marker of outcome in HD patients is body temperature (BT). However, in other diseases, such as acute heart failure and community acquired pneumonia, a relation between low BT and adverse outcome was observed ${ }^{2-4}$.

A frequent observation in the earlier days of dialysis therapy was a relatively low BT in uremic patients, so-called uremic hypothermia. The pathogenesis of this phenomenon is still uncertain; however, factors such as yet unidentified uremic cryogens and a reduced activity of Na-K-ATPase ${ }^{5-7}$ have been implemented in its pathogenesis. In contrast to earlier studies ${ }^{8,9}$, a recent report showed a higher oral temperature in dialysis patients as compared to healthy controls, but determinants of BT were not assessed in detail ${ }^{10}$.

There is abundant literature with regard to the relation between acute intra-dialytic BT changes and the hemodynamic response during dialysis ${ }^{11-13}$. However, there is a paucity of data regarding the determinants of BT in HD patients, its long-term course and potential prognostic implications.

Aims of the present study were to identify prognostic implications of trends in BT in incident HD patients.

\section{Methods}

\section{Patient Selection}

The study population included 12,695 incident HD patients who started their in-center treatment between January 1, 2000 and February 28, 2010 in 51 facilities operated by Renal Research Institute (RRI) and New York Dialysis Services (New York, NY, USA). The clinics are primarily concentrated in New York State with some clinics located in North Carolina, Michigan, Connecticut, Illinois, and California. Patients with data on demographic characteristics (date of birth, gender, race, ethnicity, diabetic status, and 
height); patients with at least 13 treatments in the first year of HD; patients whose last treatment occurred within 30 days prior to discharge date or end of year one; patients who had more than $50 \%$ of their treatments in the same dialysis shift; and those with at least one albumin draw and urea kinetic modeling done in year one were included $(n=7003)$. Patients with thyroid disease were also excluded $(n=261)$. 6742 patients were included in the final analysis. Patients' co-morbid conditions were assessed using CMS classification for co-morbid conditions ${ }^{14}$. Patients who left the clinic, switched modality, got a transplant, were with the facility beyond the end of the observation period (February 28, 2010) within the first year were censored; patients who survived the first year were also censored at 365 days. Time to death analysis was conducted for censored patients and those who died within the first 365 days of all cause mortality.

Pre-dialysis BT (measured by appropriately trained health care professionals) was recorded in clinics prior to the start of each treatment; an average of pre-dialysis BT for the first 30 days of HD was computed per patient. The slope of pre-dialysis BT change was computed by linear regression using all temperature values in year one or until censoring or death on a per patient basis irrespective of the p-value for the slope. Additional sensitivity analysis was conducted excluding first and last 12 temperature values in years 1 and 2 .

BT are measured in all RRI clinics using two types of thermometers: 3M Tempa-DOT oral (3M) and Braun Welch Allyn (Welch Allyn) ear thermometers. In a previous study we performed a validation study on the two types of thermometers ${ }^{15}$. Both temperature methods comply to ASTM (American Society for Testing and Materials) requirements. Maximum error is within $0.2^{\circ} \mathrm{C}$ for the Braun thermometer and within $0.1^{\circ} \mathrm{C}$ for the Tempa-Dot thermometer. For that study the coefficient of variation in pre-dialytic temperature using the two devices was calculated. For the Tempa-Dot thermometer, a coefficient of variation (CV) of 0.0096 (0.96\%) was observed, whereas the CV was $0.0086(0.86 \%)$ for the Braun thermometer. The same thermometer model is used within each clinic; therefore, the analysis of pre-dialysis BTs is adjusted by clinic to account for this difference. Dialysis temperature and composition was kept constant during the observation period.

Room temperature in the clinics is climate controlled, but may sometimes be slightly adjusted in response to preferences of patients and/or staff. Thus, some variability between clinics is possible given clinic specific policies within federal regulations (section V.405 of Centre for Medicare and Medicaid services; ESRD interpretative guidance section 1.1, dated October 4,2008$)$. However, this variability is accounted for by adjustment by clinic. 
RRI follows ultrapure AAMI standards in water and dialysate quality. As such, all clinics studied achieved between 80 to $100 \%$ of the dialysate $L A L<0.03$. However, it should be noted that data on LAL and dialysate cultures are only available in the database since 2008. Given the fact that pre-dialysis BT, and not intra-dialytic changes were studied, results for microbiological dialysate quality are not included in the analysis.

Patients were stratified according to tertiles of pre-dialysis BT in the first 30 days (Tertile 1: $<=36.47^{\circ} \mathrm{C}$, Tertile 2 : $>36.47$ to $36.71^{\circ} \mathrm{C}$, Tertile $3:>36.7^{\circ} \mathrm{C}$ ) and according to temperature slope (increased: $>0.01^{\circ} \mathrm{C} /$ month, decreased: $<-0.01^{\circ} \mathrm{C} /$ month, and stable, with change between -0.01 and $+0.01^{\circ} \mathrm{C} /$ month). This resulted in nine distinct groups based on starting pre-dialysis temperature and slopes.

Other repeated measures such as pre-dialysis systolic and diastolic blood pressures (pre-SBP and pre-DBP, respectively), inter-dialytic weight gain (IDWG), post-dialysis weight, albumin, enPCR, eKdrt/V, and creatinine were computed as an average of all values in the first year or until censoring or death. All labs were measured by Spectra Laboratories (Rockleigh, NJ, USA). Total neutrophil count, as a surrogate parameter of inflammation ${ }^{16}$, was available on a monthly basis in $64 \%$ of the population.

To validate our findings, we conducted an analysis of patients who survived up to the beginning of the second year of HD. We repeated our methodology by stratifying the patients according to the BTs in the first 30 days of year two and according to the BT slope in the second year.

This analysis of the RRI database was approved by the Beth Israel Medical Center IRB.

\section{Statistical analysis}

The nine pre-dialysis BT and BT slope groups were compared using analysis of variance (ANOVA). Similar comparison was conducted for three groups of baseline BT and three groups of BT slopes. Mortality analysis was conducted using unadjusted KaplanMeier analysis as well as Cox proportional hazards model adjusted for gender, age, race, ethnicity, co-morbid conditions, BMI, pre-SBP and pre-DBP, IDWG, post-dialysis weight, urea distribution volume, albumin, $\mathrm{nPCR}$, eKdrt/V, creatinine, residual renal function, access type, individual clinic, season in which patient started treatment, and dialysis shift. In a subgroup analysis, an adjustment for the slope in total neutrophil count was performed.

All $p$-values are two-sided. The analyses were conducted in SPSS version 18 (IBM, Somers, NY) and SAS version 9.2 (SAS Institute, Cary, NC). 


\section{Results}

\section{Demographics}

6742 Patients with an average age of 62.1 (SD 15.5) years were studied. Patient characteristics are displayed in Table 5.1. Overall, patients started with an average BT of $36.57^{\circ} \mathrm{C}$ and experienced a drop of $0.009^{\circ} \mathrm{C}$ per month.

Table 5.1 Patient characteristics - by beginning body temperature.

\begin{tabular}{|c|c|c|c|c|c|c|}
\hline & & $\begin{array}{c}\text { Tertile } 1 \\
\left(\leq 36.47^{\circ} \mathrm{C}\right)\end{array}$ & $\begin{array}{c}\text { Tertile } 2 \\
(>36.47 \text { to } \\
36.71^{\circ} \mathrm{C}\end{array}$ & $\begin{array}{l}\text { Tertile } 3 \\
\left(>36.7^{\circ} \mathrm{C}\right)\end{array}$ & Total & p-values \\
\hline \multicolumn{2}{|l|}{$\mathrm{N}$} & 2251 & 2250 & 2241 & 6742 & \\
\hline \multicolumn{2}{|c|}{ Pre-dialysis Temperature Slope (per month) } & $0.007(0.072)$ & $-0.009(0.051)$ & $-0.023(0.063)$ & $-0.009(0.064)$ & $<0.001$ \\
\hline \multicolumn{2}{|c|}{ Pre-dialysis Temperature at baseline $(\mathrm{t})$} & $36.2(0.26)$ & $36.6(0.07)$ & $36.9(0.18)$ & $36.57(0.34)$ & $<0.001$ \\
\hline \multicolumn{2}{|l|}{$\%$ Male } & $60 \%$ & $58 \%$ & $54 \%$ & $57 \%$ & $<0.001$ \\
\hline \multicolumn{2}{|l|}{ Age } & $65.7(15.3)$ & $61.7(15.3)$ & $58.3(15.1)$ & $61.9(15.5)$ & $<0.001$ \\
\hline \multirow[t]{3}{*}{ Race } & \% Black & $27 \%$ & $38 \%$ & $53 \%$ & $39 \%$ & $<0.001$ \\
\hline & $\%$ White & $65 \%$ & $54 \%$ & $38 \%$ & $52 \%$ & $<0.001$ \\
\hline & \%Asian & $3 \%$ & $2 \%$ & $2 \%$ & $2 \%$ & 0.028 \\
\hline Ethnicity & $\%$ Hispanic & $10 \%$ & $15 \%$ & $15 \%$ & $13 \%$ & $<0.001$ \\
\hline \multirow[t]{12}{*}{ Comorbids } & $\%$ Diabetic & $56 \%$ & $54 \%$ & $51 \%$ & $54 \%$ & 0.033 \\
\hline & $\%$ with Cancer (other than skin & $4 \%$ & $3 \%$ & $3 \%$ & $3 \%$ & $<0.001$ \\
\hline & $\%$ with Cardiac Dysrhythmias & $7 \%$ & $3 \%$ & $2 \%$ & $4 \%$ & $<0.001$ \\
\hline & $\%$ with Cerebrovascular Disease & $5 \%$ & $2 \%$ & $3 \%$ & $3 \%$ & $<0.001$ \\
\hline & $\%$ with Congestive Heart Failure & $17 \%$ & $11 \%$ & $9 \%$ & $12 \%$ & $<0.001$ \\
\hline & $\%$ with COPD & $5 \%$ & $4 \%$ & $3 \%$ & $4 \%$ & 0.003 \\
\hline & $\%$ with HIV or AIDS & $1 \%$ & $2 \%$ & $4 \%$ & $2 \%$ & $<0.001$ \\
\hline & $\%$ with Hyperparathyroidism & $2 \%$ & $1 \%$ & $1 \%$ & $1 \%$ & 0.003 \\
\hline & $\%$ with Infection & $5 \%$ & $3 \%$ & $3 \%$ & $4 \%$ & $<0.001$ \\
\hline & $\%$ with Ischemic Heart Disease & $13 \%$ & $8 \%$ & $7 \%$ & $9 \%$ & $<0.001$ \\
\hline & $\%$ with MI (incl Cardiac Arrest) & $3 \%$ & $2 \%$ & $2 \%$ & $2 \%$ & 0.006 \\
\hline & $\%$ with PVD or PAD & $10 \%$ & $5 \%$ & $5 \%$ & $7 \%$ & $<0.001$ \\
\hline \multicolumn{2}{|l|}{ BMI } & $27(9.5)$ & $27.9(10.8)$ & $28.4(9.2)$ & $27.8(9.9)$ & $<0.001$ \\
\hline \multicolumn{2}{|c|}{ Pre-dialysis Systolic Blood Pressure } & $146.9(18.8)$ & $149.5(17.9)$ & $150.8(17.5)$ & $149.1(18.1)$ & $<0.001$ \\
\hline \multicolumn{2}{|c|}{ Pre-dialysis Diastolic Blood Pressure } & $75.2(11.1)$ & $77.8(10.8)$ & $79.6(10.6)$ & $77.5(10.9)$ & $<0.001$ \\
\hline \multicolumn{2}{|c|}{ Inter-dialytic Weight Gain (\% of Post-dialysis Weight } & $3.17(1.02)$ & $3.16(0.98)$ & $3.15(0.96)$ & $3.16(0.99)$ & 0.75 \\
\hline \multicolumn{2}{|c|}{ Post-dialysis Weight } & $75.1(20.2)$ & $77.1(20.6)$ & $78.6(21.4)$ & $77(20.8)$ & $<0.001$ \\
\hline \multicolumn{2}{|c|}{ Urea distribution volume } & $39(8.3)$ & $40(8.9)$ & $40.4(9.2)$ & $39.8(8.8)$ & $<0.001$ \\
\hline \multicolumn{2}{|l|}{ Albumin } & $3.68(0.42)$ & $3.72(0.44)$ & $3.72(0.45)$ & $3.71(0.43)$ & 0.004 \\
\hline \multicolumn{2}{|l|}{ enPCR } & $0.85(0.21)$ & $0.86(0.2)$ & $0.85(0.2)$ & $0.86(0.21)$ & 0.074 \\
\hline \multicolumn{2}{|l|}{ eKdrt/V } & $1.4(0.35)$ & $1.38(0.29)$ & $1.35(0.29)$ & $1.38(0.31)$ & $<0.001$ \\
\hline \multicolumn{2}{|l|}{ Creatinine } & $6.66(2.48)$ & $7.35(2.72)$ & $7.98(2.84)$ & $7.33(2.74)$ & $<0.001$ \\
\hline \multicolumn{2}{|c|}{$\%$ with Residual Renal Function at baseline } & $35 \%$ & $36 \%$ & $29 \%$ & $33 \%$ & $<0.001$ \\
\hline \multicolumn{2}{|c|}{$\%$ with AVF at start of dialysis } & $19 \%$ & $20 \%$ & $20 \%$ & $20 \%$ & 0.473 \\
\hline \multicolumn{2}{|c|}{$\%$ with AVG at start of dialysis } & $7 \%$ & $7 \%$ & $9 \%$ & $8 \%$ & 0.003 \\
\hline \multicolumn{2}{|c|}{$\%$ with Catheter at start of dialysis } & $74 \%$ & $73 \%$ & $70 \%$ & $72 \%$ & 0.016 \\
\hline \multicolumn{2}{|c|}{$\%$ with TempoDot thermometers } & $84 \%$ & $90 \%$ & $94 \%$ & $89 \%$ & $<0.001$ \\
\hline \multicolumn{2}{|c|}{$\%$ treated in morning } & $30 \%$ & $27 \%$ & $26 \%$ & $28 \%$ & 0.007 \\
\hline \multicolumn{2}{|c|}{$\%$ treated in afternoon } & $32 \%$ & $30 \%$ & $30 \%$ & $30 \%$ & 0.044 \\
\hline \multicolumn{2}{|c|}{$\%$ treated in evening } & $6 \%$ & $12 \%$ & $16 \%$ & $11 \%$ & $<0.001$ \\
\hline
\end{tabular}




\section{Relation between baseline body temperature, co-morbid conditions and other patient characteristics}

Patients who started with lower BTs (tertile 1) were more likely to experience an increase in their $\mathrm{BT}$ (average increase: $0.006^{\circ} \mathrm{C}$ per month) while patients who started with higher BTs were more likely to experience a decline in body temperature in the first year (average decline: $0.023^{\circ} \mathrm{C}$ per month). In an analysis of demographic characteristics, patients with higher BTs were more likely to be female (46\%), younger ( 58.3 years), twice more likely to be of black race than patients with lower BTs (53\% versus $27 \%$ ) and more likely to be of Hispanic ethnicity (Table 5.1). In an analysis of comorbid conditions, patients with higher starting BTs compared to patients in tertile 1, were less likely to have arrhythmias, cerebrovascular disease, cancer, congestive heart failure (CHF), ischemic heart disease (IHD), chronic obstructive pulmonary disease, whereas a lower percentage had a history of myocardial infarction (MI), and peripheral vascular/arterial disease (PVD/PAD). At the same time, patients with higher BTs were more likely to have HIV/AIDS than patients with lower BTs (Table 5.1).

Patients with higher starting BTs are also more likely to be larger (average BMI $28.4 \mathrm{~kg} / \mathrm{m}^{2}$ ), have higher pre-SBP and pre-DBP (150.8 and $79.6 \mathrm{mmHg}$, respectively), have higher albumin $(3.72 \mathrm{~g} / \mathrm{dl})$, higher creatinine $(7.98 \mathrm{~g} / \mathrm{dl})$, less likely to have residual renal function at the start of HD (29\%) and slightly less likely to have catheters at start of HD (71\%) (Table 5.1).

\section{Relation between change in body temperature, co-morbid conditions and other patient characteristics}

2093 Patients experienced a decline in BT (43\%); 2238 patients remained steady (33\%); and 1601 experienced an increase in BT (24\%). In an analysis of demographic characteristics, patients whose BT increased were more likely to be male (61\%). Patients with stable BTs were more likely to be of Black race. Notably no significant differences existed in age and ethnicity between groups of BT patterns (Table 5.2).

In an analysis of co-morbid conditions, patients with stable BTs compared to other two groups, were less likely to have arrhythmias and CHF. Patients with stable BTs were also more likely to have higher pre-SBP and pre-DBP (150.2 and $77.8 \mathrm{mmHg}$ ), have higher IDWG (3.2\%), have higher albumin $(3.75 \mathrm{~g} / \mathrm{dl})$, higher nPCR $(0.87 \mathrm{~g} / \mathrm{kg} / \mathrm{day})$, higher creatinine $(7.53 \mathrm{mg} / \mathrm{dl})$, and more likely to have residual renal function at the start of dialysis (36\%) (Table 5.2). 
Table 5.2 Patient characteristics - by body temperature slope.

\begin{tabular}{|c|c|c|c|c|c|c|}
\hline & & $\begin{array}{l}\text { Declined } \\
\text { (drop } \\
\leq-0.01^{\circ} \mathrm{C} / \\
\text { mnth) }\end{array}$ & $\begin{array}{c}\text { Steady } \\
\text { (change }-0.01 \\
\text { to } 0.01^{\circ} \mathrm{C} \text { / } \\
\text { mnth }\end{array}$ & $\begin{array}{l}\text { Increased } \\
\text { (increases } \\
\geq 0.01^{\circ} \mathrm{C} \text { ) }\end{array}$ & Total & $\mathrm{p}$-values \\
\hline \multicolumn{2}{|l|}{$\mathrm{N}$} & 2903 & 2238 & 1601 & 6742 & \\
\hline \multicolumn{2}{|c|}{ Pre-dialysis Temperature Slope (per month) } & $-0.044(0.06)$ & $-0.001(0.006)$ & $-0.044(0.074)$ & $-0.009(0.064)$ & $<0.001$ \\
\hline \multicolumn{2}{|c|}{ Pre-dialysis Temperature at baseline $(\mathrm{t})$} & $36.65(0.33)$ & $36.58(0.29)$ & $36.39(0.37)$ & $36.57(0.34)$ & $<0.001$ \\
\hline \multicolumn{2}{|l|}{$\%$ Male } & $56 \%$ & $57 \%$ & $61 \%$ & $57 \%$ & 0.002 \\
\hline \multicolumn{2}{|l|}{ Age } & $61.7(15.4)$ & $61.8(15.4)$ & $62.5(15.9)$ & $61.9(15.5)$ & 0.239 \\
\hline \multirow[t]{3}{*}{ Race } & \% Black & $39 \%$ & $42 \%$ & $36 \%$ & $39 \%$ & $<0.001$ \\
\hline & $\%$ White & $53 \%$ & $49 \%$ & $36 \%$ & $39 \%$ & $<0.001$ \\
\hline & \%Asian & $2 \%$ & $3 \%$ & $2 \%$ & $2 \%$ & 0.285 \\
\hline Ethnicity & \% Hispanic & $14 \%$ & $13 \%$ & $12 \%$ & $13 \%$ & 0.412 \\
\hline \multirow[t]{12}{*}{ Comorbids } & $\%$ Diabetic & $52 \%$ & $56 \%$ & $54 \%$ & $54 \%$ & 0.069 \\
\hline & $\%$ with Cancer (other than skin & $3 \%$ & $3 \%$ & $3 \%$ & $3 \%$ & 0.643 \\
\hline & $\%$ with Cardiac Dysrhythmias & $4 \%$ & $3 \%$ & $5 \%$ & $4 \%$ & 0.006 \\
\hline & $\%$ with Cerebrovascular Disease & $4 \%$ & $3 \%$ & $4 \%$ & $3 \%$ & 0.213 \\
\hline & $\%$ with Congestive Heart Failure & $13 \%$ & $11 \%$ & $13 \%$ & $12 \%$ & $<0.001$ \\
\hline & $\%$ with COPD & $4 \%$ & $4 \%$ & $4 \%$ & $4 \%$ & 0.003 \\
\hline & $\%$ with HIV or AIDS & $2 \%$ & $2 \%$ & $2 \%$ & $2 \%$ & $<0.001$ \\
\hline & $\%$ with Hyperparathyroidism & $2 \%$ & $1 \%$ & $2 \%$ & $1 \%$ & 0.003 \\
\hline & $\%$ with Infection & $4 \%$ & $4 \%$ & $4 \%$ & $4 \%$ & $<0.001$ \\
\hline & $\%$ with Ischemic Heart Disease & $10 \%$ & $9 \%$ & $9 \%$ & $9 \%$ & $<0.001$ \\
\hline & $\%$ with MI (incl Cardiac Arrest) & $2 \%$ & $2 \%$ & $2 \%$ & $2 \%$ & 0.006 \\
\hline & $\%$ with PVD or PAD & $7 \%$ & $7 \%$ & $6 \%$ & $7 \%$ & $<0.001$ \\
\hline \multicolumn{2}{|l|}{ BMI } & $27.7(9.7)$ & $28.2(11)$ & $27.5(8.4)$ & $27.8(9.9)$ & $<0.001$ \\
\hline \multicolumn{2}{|c|}{ Pre-dialysis Systolic Blood Pressure } & $149.1(18.3)$ & $150.2(17.7)$ & $147.4(18.4)$ & $149.1(18.1)$ & $<0.001$ \\
\hline \multicolumn{2}{|c|}{ Pre-dialysis Diastolic Blood Pressure } & $77.8(11)$ & $77.8(10.6)$ & $76.7(11.3)$ & $77.5(10.9)$ & $<0.001$ \\
\hline \multicolumn{2}{|c|}{ Inter-dialytic Weight Gain (\% of Post-dialysis Weight } & $3.13(1)$ & $3.21(0.94)$ & $3.13(1.03)$ & $3.16(0.99)$ & 0.75 \\
\hline \multicolumn{2}{|c|}{ Post-dialysis Weight } & $76.6(20.6)$ & $77.4(20.9)$ & 77 (20.9) & $77(20.8)$ & $<0.001$ \\
\hline \multicolumn{2}{|c|}{ Urea distribution volume } & $39.7(8.9)$ & $39.7(8.8)$ & $40.1(8.8)$ & $39.8(8.8)$ & $<0.001$ \\
\hline \multicolumn{2}{|l|}{ Albumin } & $3.68(0.45)$ & $3.75(0.39)$ & $3.68(0.46)$ & $3.71(0.43)$ & 0.004 \\
\hline \multicolumn{2}{|l|}{ enPCR } & $0.85(0.21)$ & $0.87(0.19)$ & $0.85(0.22)$ & $0.86(0.21)$ & 0.074 \\
\hline \multicolumn{2}{|l|}{ eKdrt/V } & $1.37(0.3)$ & $1.38(0.29)$ & $1.38(0.36)$ & $1.38(0.31)$ & $<0.001$ \\
\hline \multicolumn{2}{|l|}{ Creatinine } & $7.31(2.77)$ & $7.53(2.7)$ & $7.1(2.72)$ & $7.33(2.74)$ & $<0.001$ \\
\hline \multicolumn{2}{|c|}{$\%$ with Residual Renal Function at baseline } & $32 \%$ & $36 \%$ & $32 \%$ & $32 \%$ & $<0.001$ \\
\hline \multicolumn{2}{|c|}{$\%$ with AVF at start of dialysis } & $19 \%$ & $21 \%$ & $19 \%$ & $19 \%$ & 0.473 \\
\hline \multicolumn{2}{|c|}{$\%$ with AVG at start of dialysis } & $8 \%$ & $9 \%$ & $7 \%$ & $7 \%$ & 0.003 \\
\hline \multicolumn{2}{|c|}{$\%$ with Catheter at start of dialysis } & $73 \%$ & $71 \%$ & $73 \%$ & $73 \%$ & 0.016 \\
\hline \multicolumn{2}{|c|}{$\%$ with TempoDot thermometers } & $89 \%$ & $91 \%$ & $87 \%$ & $87 \%$ & $<0.001$ \\
\hline \multicolumn{2}{|c|}{$\%$ treated in morning } & $29 \%$ & $27 \%$ & $26 \%$ & $26 \%$ & 0.007 \\
\hline \multicolumn{2}{|c|}{$\%$ treated in afternoon } & $30 \%$ & $29 \%$ & $33 \%$ & $33 \%$ & 0.044 \\
\hline \multicolumn{2}{|c|}{$\%$ treated in evening } & $11 \%$ & $12 \%$ & $12 \%$ & $11 \%$ & $<0.001$ \\
\hline
\end{tabular}




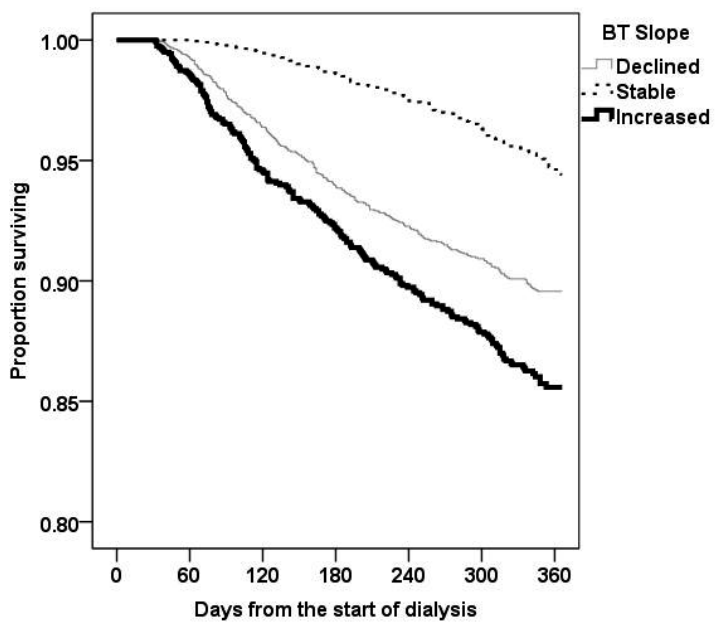

Figure 5.1 Survival by body temperature slope.

Table 5.3 Cox proportional hazards results for year 1 (only significant predictors are shown).

\begin{tabular}{|c|c|c|c|c|}
\hline & \multirow[b]{2}{*}{ Hazard Ratio } & \multicolumn{2}{|c|}{$95.0 \% \mathrm{Cl}$ for $\mathrm{HR}$} & \multirow[b]{2}{*}{ Sig. } \\
\hline & & Lower & Upper & \\
\hline Tertile 1, Decline & 1.731 & 1.185 & 2.528 & 0.005 \\
\hline Tertile 2, Decline & 1.766 & 1.232 & 2.532 & 0.002 \\
\hline Tertile 3, Decline & 1.463 & 1.025 & 2.088 & 0.036 \\
\hline Tertile 1, Stable & 0.962 & 0.625 & 1.481 & 0.860 \\
\hline \multicolumn{5}{|l|}{ Tertile 2, Stable } \\
\hline Tertile 3, Stable & 0.500 & 2.286 & 0.874 & 0.015 \\
\hline Tertile 1 , Increased & 2.175 & 1.531 & 3.089 & 0.000 \\
\hline Tertile 2, Increased & 2.265 & 1.524 & 3.366 & 0.000 \\
\hline Tertile 3, Increased & 2.028 & 1.223 & 3.361 & 0.006 \\
\hline Male & 1.350 & 1.089 & 1.673 & 0.006 \\
\hline Age & 1.020 & 1.012 & 1.029 & 0.000 \\
\hline Infection & 0.402 & 0.198 & 0.816 & 0.012 \\
\hline Ischemic heart disease & 0.588 & 0.396 & 0.873 & 0.008 \\
\hline Myocardaial infarction & 2.174 & 1.086 & 4.352 & 0.028 \\
\hline Pre-dialysis SBP & 0.983 & 0.976 & 0.991 & 0.000 \\
\hline Albumin & 0.201 & 0.167 & 0.242 & 0.000 \\
\hline Craetinine & 0.835 & 0.789 & 0.885 & 0.000 \\
\hline Residual renal function at baseline & 0.486 & 0.375 & 0.629 & 0.000 \\
\hline AVG at start of dialysis & 0.672 & 0.453 & 0.997 & 0.048 \\
\hline Treated in other shift & 1.376 & 1.070 & 1.771 & 0.013 \\
\hline Treated in evening shift & 2.092 & 1.507 & 2.903 & 0.000 \\
\hline
\end{tabular}

Other covariates in the model omitted with $p>0.05$ : White race, Asian race, Hispanic ethnicity, diabetes, anemias, cancer, cardiac dysrhythmias, cerebrovascular disease, CHF, chronic obstructive pulmonary disease, drug or alcohol dependence, gastrointestinal bleeding, hepatitis, HIV/AIDS, hyperparathyroidism, peripheral vascular/arterial disease, pneumonia, BMI, IDWG, post-dialysis weight, pre-dialysis DBP, urea distribution volume, enPCR, treated in afternoon shift, started in spring, summer, or fall, clinic location Reference group: race=black; ethnicity=non-Hispanic; access type=catheter; treated in morning shift; started dialysis in winter 


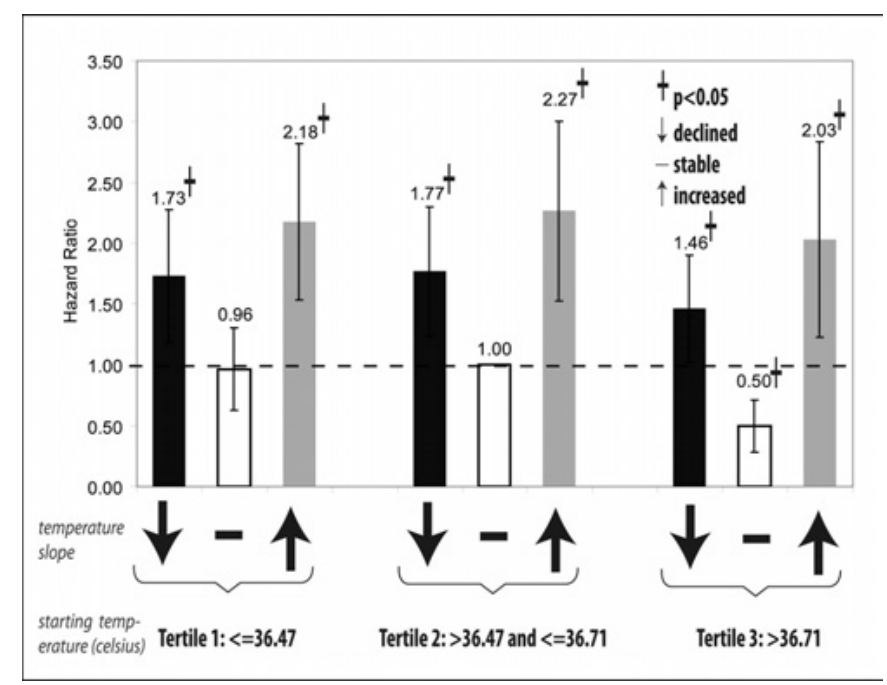

Figure 5.2 Cox proportional hazards results in year 1.

These data were confirmed by an analysis of patients in the second year after starting dialysis treatment, which showed comparable results as the first year after starting dialysis (Figure 5.3). Also, a subgroup analysis in patients in whom total neutrophil counts were available showed no material changes in hazard ratios after adjustment for the change in neutrophil count (hazard ratio in patients with declining temperature were 2.69, 2.07, and 1.97 in temperature tertiles 1,2 , and 3 respectively; in patients with increasing temperature hazard ratios were 3.16, 3.24, and 2.25 in temperature tertiles 1,2 , and 3 respectively).

In an analysis of causes of deaths in the nine groups, patients who started with lower BTs were more likely to die of cardiovascular reasons than other patients. Patients who started with higher BTs (tertile 3 ) were more likely to die of infections or neoplasms; notably, $11 \%$ of patients who started with $\mathrm{BT}>36.71^{\circ} \mathrm{C}$ and declined died of neoplasms, which is significantly higher than the overall population where only $5 \%$ of patients died of neoplasms. In patients who started with normal BTs (>36.47 to $36.71^{\circ} \mathrm{C}$ ), distribution of causes of deaths varied greatly across patterns of BT; patients whose temperature remained steady were more likely to die of CVD causes $(75 \%$ compared to overall population of $61 \%$ ), while patients whose BT increased in the first year were more likely to die of infection-related causes (29\% compared to overall population of $14 \%$ ) (Table 5.4 ). 


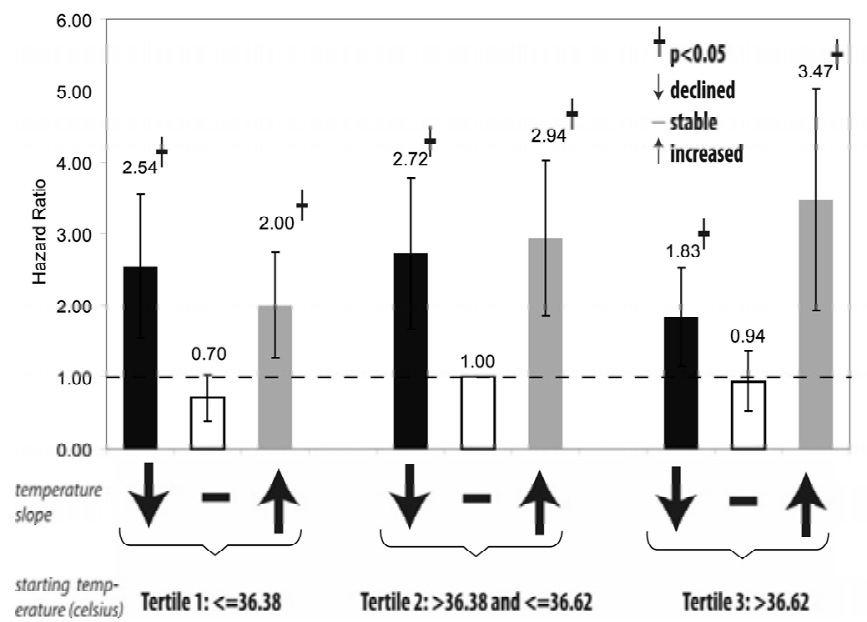

Figure 5.3 Cox proportional hazards results in year 2.

Table 5.4 Causes of death.

\begin{tabular}{lcccc}
\hline & CVD & Infections & Neoplasm & Other \\
\hline Tertile 1, Declined & $57 \%$ & $13 \%$ & $5 \%$ & $25 \%$ \\
Tertile 2, Declined & $55 \%$ & $14 \%$ & $5 \%$ & $26 \%$ \\
Tertile 3, Declined & $55 \%$ & $16 \%$ & $11 \% *$ & $18 \%$ \\
Tertile 1, Steady & $69 \%$ & $5 \%$ & $0 \%$ & $26 \%$ \\
Tertile 2, Steady & $75 \% *$ & $6 \%$ & $4 \%$ & $15 \%$ \\
Tertile 3, Steady & $56 \%$ & $11 \%$ & $11 \%$ & $22 \%$ \\
Tertile 1, Increased & $68 \%$ & $13 \%$ & $3 \%$ & $16 \%$ \\
Tertile 2, Increased & $55 \%$ & $29 \% *$ & $2 \%$ & $14 \%$ \\
Tertile 3, Increased & $58 \%$ & $19 \%$ & $4 \%$ & $19 \%$ \\
Overall population & $61 \%$ & $14 \%$ & $5 \%$ & $20 \%$ \\
\hline
\end{tabular}

$* p<0.05$ comparing given percentage with overall population

In an analysis of temporal trends of BT before death, we observed that declines and increases in BT appear to be more chronic, long term phenomena, rather than acute decrease or increase immediately preceding death. We assessed this relationship by fitting different curves to BTs before death and linear relationship described it with highest r-squared (Figure 5.4 and 5.5). 


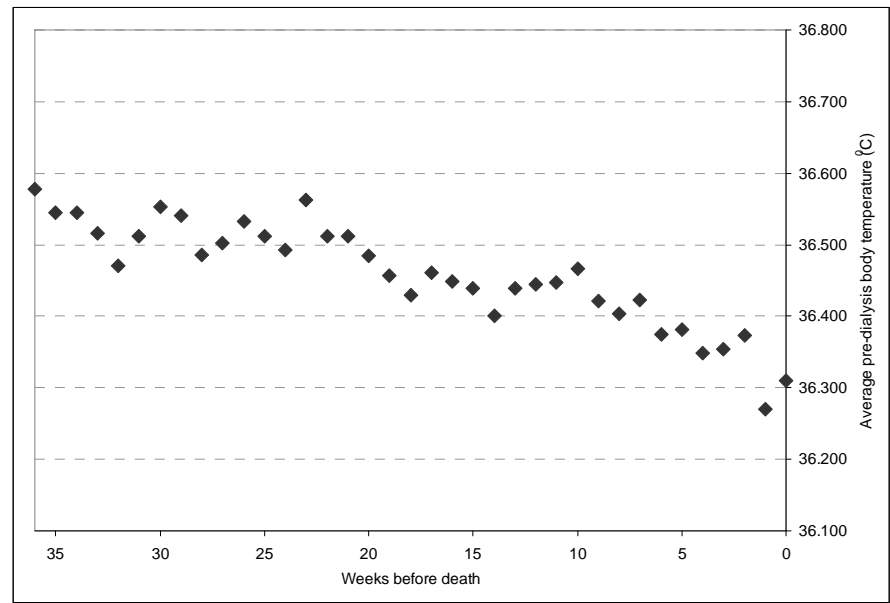

Figure 5.4 Mean weekly body temperatures prior to death in patients whose body temperatures declined.

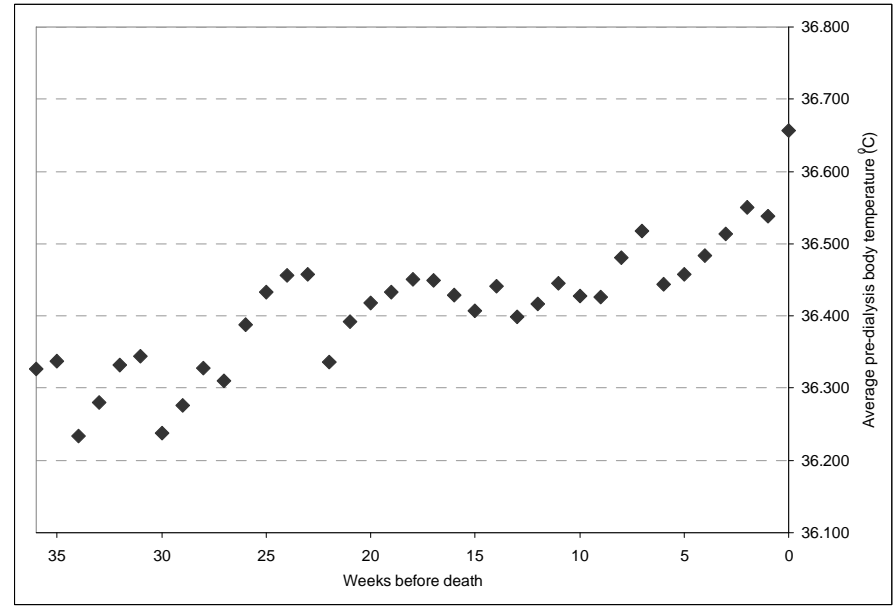

Figure 5.5 Mean weekly body temperatures prior to death in patients whose body temperatures increased. 


\section{Discussion}

The main finding of the present study is the association between trends in pre-dialytic BT and outcome in chronic HD patients. The highest mortality was observed in the cohorts in whom BT increased or decreased during the follow-up period, irrespective of the baseline BT at baseline. The lowest mortality was observed in the group with the highest BT at baseline and with stable BTs during the follow-up period. To the best of our knowledge, this is the first study in which the relation between BT and outcome was studied in HD patients. Moreover, as compared to many epidemiological studies in dialysis patients, this analysis is not only cross-sectional but takes longitudinal trends into account.

BT at baseline appeared to be related to various factors. The tertile with the lowest BT was characterized by a higher percentage of males and white patients, and higher age, where nutritional parameters, such as BMI, and serum creatinine were lower as compared to the reference group. Moreover, the relative percentage of patients with various co-morbid conditions was higher in the lowest tertile. This is in contrast to studies in animal models, in which low BT were related to longevity ${ }^{17}$.

Patients with higher starting BTs appeared to be more likely to experience a decline in their temperature in the first year while patients with the lower BTs experienced an increase in their temperature, raising the possibility of "regression to the mean" phenomenon. Although this is a possibility, this is unlikely because of the associated higher mortality in these groups as well as because the results were similar in the second year. Interestingly, there was an average decline in BT in the entire population possibly brought about by a change in inflammatory status due to initiation of HD.

The percentage of patients with "uremic hypothermia" at baseline cannot be deduced from the study due to the absence of a control group with healthy subjects. However, mean values for the cohort do not appear to differ widely from values reported in the literature for the general population ${ }^{18}$, although timing of the measurements was variable in our study, depending on the dialysis shift. Notably, residual renal function was highest in the middle, and not in the highest tertile.

The results of the present study suggest that a lower pre-dialytic BT might be, to some degree, a marker of co-morbidity, but is also related to other factors such as age, race and BMI. In a multivariate analysis gender, age, race, as well as various comorbid conditions were all significant predictors of BT. The lower BT in males as compared to females and the inverse relation between age and temperature is in agreement with data from the general population ${ }^{19,20}$. However, in the general population, the relation between BMI and BT is controversial. Whereas some studies found BMI to be 
inversely related to $\mathrm{BT}^{19}$, others observed either no relationship ${ }^{21}$, or a positive relation between $\mathrm{BMI}$ and $\mathrm{BT}^{22}$.

Despite the relation with co-morbidity, in the Cox regression model, baseline predialytic BT did not appear to emerge as a powerful independent risk factor, although the data of this study suggest that mortality is lowest in the group with the highest pre-dialytic BT at baseline. Trends for BT appeared to be more strongly related to outcome than baseline temperature per se. This held true for an increasing, as well as a decreasing trend, which was independently associated with an increased risk for mortality, independently of baseline BT.

When dividing the study group within 3 cohorts depending on the trend in BT, the cohort with the increase in BT was characterized by a lower serum albumin as well as lower serum creatinine levels as compared to the group with stable BT, which might point at a malnourished or inflammatory state, or both. Although C-reactive protein levels were not assessed, it might be hypothesized that the increase in BT could be explained by a stimulation of the acute phase response ${ }^{23}$.

More intriguing appears the fact that the groups with a decreasing temperature had an increased risk of mortality as compared to the groups with stable BT. Characteristics for the group in whom BT declined during the follow up period were comparable to the group with an increase in $\mathrm{BT}$, which is congruent with a $\mathrm{U}$ shaped phenomenon. With the parameters used in the present analysis, we were not able to establish parameters which could predict why patients experience a decline as compared to an increase in BT. In an analysis of trends in BT before death, we observed that declines and increases in BT appear to be more chronic, long term phenomena, rather than acute drop or increase immediately preceding death. This held true for both patient groups with an increase, as well as a decrease BT before death.

Thus, the causes behind the relation between the decline in BT and adverse outcome cannot be deduced from the present data. It has been hypothesized that a reduction in BT might be an adaptive response of the body to inflammation when the body cannot cope with the increasing demands of fever, e.g. in cases of insufficient nutrition ${ }^{24}$. This might coincide with the non-thyroidal illness syndrome, characterized by a lowering of the metabolic state and reduction of T3 levels ${ }^{25}$. Recently, a relation between low free T3 levels and mortality was observed in dialysis patients ${ }^{26}$.

Several cryogenic factors, such as $\mathrm{MSH}$ and neuropeptide $\mathrm{Y}$, as well as a reduced $\mathrm{Na}-\mathrm{K}$ ATPase activity, which may be influenced by both renal failure as well as inflammation, might play a role in changes in core temperature in dialysis 
patients $^{23-25,27-31}$. However, the role of these putative factors should be assessed in future studies.

Another factor which might lead to a decrease in BT is cardiac failure. Low BT was associated with increased mortality in patients with heart failure ${ }^{2,3,32}$, but mortality from cardiac causes did not appear to be different between the subgroups of patients with increasing or lowering trends, although mortality from infectious causes was higher in one subgroup of patients with an increase in BT.

Lastly, though unlikely, a contributing effect of low ambient temperatures on both BT and outcome should be discussed. Ambient temperature likely has an effect on $\mathrm{BT}^{33}$, whereas higher mortality in winter period was recently shown by our group in dialysis patients, and also in the general population ${ }^{34,35}$. However, the relation between trends and BT remained significant after correction for season. Moreover, when looking at the trends in BT, a gradual increase or decrease in BT was observed in those patients who expired during the study period.

A major strength of this analysis is the large sample size and patients treated in 51 different dialysis clinics in different states and geographical regions. Widespread patient population and the considerably large sample size, the study cohort may be assumed generalizable to the HD population in the United States. Selection of incident patients allowed the study of the evolution of BT virtually without effects of HD treatment at study baseline. Although the results may not have an immediate impact of daily patient care, we suggest that they are of considerable interest, by showing that the dynamics of key physiological parameters in dialysis patients are profoundly related to mortality. The concept regarding longitudinal trends and mortality is a relatively novel in dialysis patients and might find future clinical application as part of a multidimensional risk model in this vulnerable group of patients.

Drawbacks of the study are firstly the use of two different methods which were used to assess BT. However, the method used had no impact on the relation between temperature trends and mortality after inclusion in the Cox model after adjusting by clinic. Secondly, timing of the BT measurement, as well as ambient temperature can also have important effects on BT. However, also consideration of these factors, by adjustment for geographical location, timing of dialysis shift and season of start dialysis, in the Cox model did not materially alter the results. Moreover, no detailed data of inflammation were available, except for serum albumin, which was lower in the cohorts with both a reduction and an increase in BT during the follow-up period. No information on T3 levels which may influence temperature were available. 


\section{Conclusion}

During a one year follow up period, both an increasing as well as a declining trend in BT was associated with an increased risk of death in incident HD patients. Prognosis was best in patients in the highest tertile for baseline BT, which remained stable during the follow-up period. Trends in BT appear to be a marker for outcome in HD patients. More detailed analysis of BT changes is needed to obtain more insight into the pathophysiologic mechanisms behind trends in BT and mortality in HD patients. 


\section{References}

1 Kalantar-Zadeh K, Abbott KC, Kronenberg F, Anker SD, Horwich TB, Fonarow GC. Epidemiology of dialysis patients and heart failure patients. Semin Nephrol. 2006;26:118-33.

2 Potapov EV, Stepanenko A, Kukucka M, Ba Fadhl FH, Qedra N, Weng Y, Pasic M, Lehmkuhl HB, Krabatsch T, Hetzer R. Prediction of survival in patients with cardiogenic shock and multiorgan failure treated with biventricular assist device. ASAIO J. 2010;56:273-8.

3 Nallamothu BK, Payvar S, Wang Y, Kosiborod M, Masoudi FA, Havranek EP, Foody JM, Casscells SW, Krumholz HM. Admission body temperature and mortality in elderly patients hospitalized for heart failure. J Am Coll Cardiol. 2006;47:2563-4.

4 Ronan D, Nathwani D, Davey P, Barlow G. Predicting mortality in patients with community-acquired pneumonia and low curb-65 scores. Eur J Clin Microbiol Infect Dis. 2010;29:1117-24.

$5 \quad$ Ash SR. An explanation for uremic hypothermia. Int J Artif Organs. 1991;14:67-9.

6 Kluger MJ, Turnbull AJ, Cranston WI, Wing AJ, Gross MP, Rothenburg BA. Endogenous cryogen excreted by the kidneys. Am J Physiol. 1981;241:R271-6.

7 Cox PS, Rothenburg BA, Kluger MJ. Characterization of an endogenous cryogen that appears in the urine. Am J Physiol. 1982;243:R241-4.

8 Fine A, Penner B. The protective effect of cool dialysate is dependent on patients' predialysis temperature. Am J Kidney Dis. 1996;28:262-5.

9 Pergola PE, Habiba NM, Johnson JM. Body temperature regulation during hemodialysis in long-term patients: Is it time to change dialysate temperature prescription? Am J Kidney Dis. 2004;44:155-65.

10 Hasan R, Adhi M, Mahmood SF, Noman F, Awan S, Akhtar F, Naqvi A, Rizvi AU. Range for normal body temperature in hemodialysis patients and its comparison with that of healthy individuals. Nephron Clin Pract. 2010;114:c303-8.

11 Maggiore Q, Pizzarelli F, Santoro A, Panzetta G, Bonforte G, Hannedouche T, Alvarez de Lara MA, Tsouras I, Loureiro A, Ponce P, Sulkova S, Van Roost G, Brink H, Kwan JT. The effects of control of thermal balance on vascular stability in hemodialysis patients: Results of the European randomized clinical trial. Am J Kidney Dis. 2002;40:280-90.

12 van der Sande FM, Kooman JP, Burema JH, Hameleers P, Kerkhofs AM, Barendregt JM, Leunissen KM. Effect of dialysate temperature on energy balance during hemodialysis: Quantification of extracorporeal energy transfer. Am J Kidney Dis. 1999;33:1115-21.

13 Rosales LM, Schneditz D, Morris AT, Rahmati S, Levin NW. Isothermic hemodialysis and ultrafiltration. Am J Kidney Dis. 2000;36:353-61.

14 Services CfDCaPUSaCfMM. Icd-9-cm international classification of diseases, ninth revision, clinical modification, sixth edition, 2008,

15 Usvyat LA, Kotanko P, van der Sande FM, Kooman JP, Carter M, Leunissen KM, Levin NW. Circadian variations in body temperature during dialysis. Nephrol Dial Transplant. 2012;27:1139-44

16 Reddan DN, Klassen PS, Szczech LA, Coladonato JA, O'Shea S, Owen WF, Jr., Lowrie EG. White blood cells as a novel mortality predictor in haemodialysis patients. Nephrol Dial Transplant. 2003;18: 1167-73.

17 Carrillo AE, Flouris AD. Caloric restriction and longevity: Effects of reduced body temperature. Ageing Res Rev. 2011;10:153-62.

18 Sund-Levander M, Forsberg C, Wahren LK. Normal oral, rectal, tympanic and axillary body temperature in adult men and women: A systematic literature review. Scand J Caring Sci. 2002;16:122-8.

19 Adam K. Human body temperature is inversely correlated with body mass. Eur J Appl Physiol Occup Physiol. 1989;58:471-5.

20 Waalen J, Buxbaum JN. Is older colder or colder older? The association of age with body temperature in 18,630 individuals. J Gerontol A Biol Sci Med Sci. 2011;66:487-92.

21 Heikens MJ, Gorbach AM, Eden HS, Savastano DM, Chen KY, Skarulis MC, Yanovski JA. Core body temperature in obesity. Am J Clin Nutr. 2011;93:963-7.

22 Eriksson H, Svardsudd K, Larsson B, Welin L, Ohlson LO, Wilhelmsen L. Body temperature in general population samples. The study of men born in 1913 and 1923. Acta Med Scand. 1985;217:347-52. 
23 Mackowiak PA. Concepts of fever. Arch Intern Med. 1998;158:1870-81.

24 Romanovsky AA, Szekely M. Fever and hypothermia: Two adaptive thermoregulatory responses to systemic inflammation. Med Hypotheses. 1998;50:219-26.

25 Koenig RJ. Modeling the nonthyroidal illness syndrome. Curr Opin Endocrinol Diabetes Obes. 2008;15:466-9.

26 Zoccali C, Mallamaci F, Tripepi G, Cutrupi S, Pizzini P. Low triiodothyronine and survival in end-stage renal disease. Kidney Int. 2006;70:523-8.

27 Airaghi L, Garofalo L, Cutuli MG, Delgado R, Carlin A, Demitri MT, Badalamenti S, Graziani G, Lipton JM, Catania A. Plasma concentrations of alpha-melanocyte-stimulating hormone are elevated in patients on chronic haemodialysis. Nephrol Dial Transplant. 2000;15:1212-6.

28 Zoccali C, Mallamaci F, Tripepi G, Benedetto FA, Parlongo S, Cutrupi S, lellamo D, Bonanno G, Rapisarda F, Fatuzzo P, Seminara G, Cataliotti A, Malatino LS. Prospective study of neuropeptide y as an adverse cardiovascular risk factor in end-stage renal disease. J Am Soc Nephrol. 2003;14:2611-7.

29 Vanholder R, De Smet R, Glorieux G, Argiles A, Baurmeister U, Brunet P, Clark W, Cohen G, De Deyn PP, Deppisch R, Descamps-Latscha B, Henle T, Jorres A, Lemke HD, Massy ZA, Passlick-Deetjen J, Rodriguez M, Stegmayr B, Stenvinkel P, Tetta C, Wanner C, Zidek W. Review on uremic toxins: Classification, concentration, and interindividual variability. Kidney Int. 2003;63:1934-43.

30 Ejderhamn J, Finkel Y, Strandvik B. NaK-Atpase activity in rectal mucosa of children with ulcerative colitis and crohn's disease. Scand J Gastroenterol. 1989;24:1121-5.

31 Stokes GS, Norris LA, Marwood JF, Johnston H, Caterson RJ. Effect of dialysis on circulating na,k atpase inhibitor in uremic patients. Nephron. 1990;54:127-33.

32 Brengelmann GL. Body temperature regulation in heart failure. Cardiologia. 1996;41:1033-43.

33 Lu SH, Dai YT. Normal body temperature and the effects of age, sex, ambient temperature and body mass index on normal oral temperature: A prospective, comparative study. Int J Nurs Stud. 2009;46:661-8.

34 Usvyat LA. Seasonal variations in body temperature: Annual Meeting of the American Society of Nephrology, 2010.

35 Cold exposure and winter mortality from ischaemic heart disease, cerebrovascular disease, respiratory disease, and all causes in warm and cold regions of Europe. The Eurowinter group. Lancet. 1997;349:1341-6. 


\section{Chapter 6}

\section{Dynamics of hospitalizations in hemodialysis patients: results from a large US provider}

Len A Usvyat, Jeroen P Kooman, Frank M van der Sande, Yuedong Wang, Franklin W Maddux, Nathan W Levin, Peter Kotanko Nephrol Dial Transplant. (accepted for publication) 


\section{Abstract}

\section{Aim}

In chronic hemodialysis (HD) patients hospitalizations result in significant costs and affect quality of life. We aimed to understand hospitalization rates before death and to identify patterns in clinical and laboratory parameters that precede the first hospitalization.

\section{Method}

We conducted two separate analyses in patients treated in the 51 US Renal Research Institute hemodialysis facilities. The first analysis involved hospitalizations before death in all HD patients who died between Jan 1 , 2007 and Dec 31, 2011. In the second analysis we studied the evolution of laboratory and treatment parameters before hospitalization in all patients with at least one hospital admission between Jan 1, 2007 and Dec 31, 2011. Patients were followed from up to six months prior to their first hospital admission. For comparison, we selected non-hospitalized patients and observed the evolution of their laboratory and treatment parameters.

Results

We studied 6262 patients in total. The first analysis encompassed 2058 deaths. In these patients, hospitalization increased from 0.32 admissions per patient-month four months before death to 1.85 in the month of death. In the second analysis we studied the first hospitalizations in 4204 patients. Irrespective of cause of hospitalization, all hospitalizations were preceded by an increase in neutrophil-to-lymphocyte ratio, a decline in hemoglobin and a reduction in effective treatment time and inter-dialytic weight gain. We noted no comparable trends in in non-hospitalized patients.

\section{Conclusion}

Hospitalization rates increase sharply before death. Months before the first hospitalization changes in clinical and laboratory parameters ensued. These results suggest that models aiming at predicting hospitalization should include indicators capturing the dynamics of relevant parameters. 


\section{Introduction}

Hospitalization rates in hemodialysis (HD) patients appear to have remained stable during the past decade, while a gradual improvement in mortality has been observed. Hospitalizations affect quality of life in HD patients ${ }^{1,2}$ and have profound economic consequences. It has been estimated that approximately one third of the total expenditure in end-stage renal disease (ESRD) patients results from hospital costs ${ }^{1,2}$. Moreover, hospitalization heralds an increased probability of death for patients. A recent study showed that the risk for death during hospitalization was increased for patients with chronic kidney disease, and even more so for HD patients as compared to the general population ${ }^{3,4}$. Clinical experience indicates that the number of hospitalizations increases before death. Therefore, the hospital admission rate is likely to be an important prognostic parameter for outcome. However, in HD patients, the relationship between the rate of hospitalizations and mortality has not yet been addressed quantitatively.

Timely identification of dialysis patients at risk for hospitalization might aid preventive strategies. Various studies have analyzed risk factors for non-elective hospital admissions both in dialysis and in pre-dialysis patients using patient characteristics, laboratory and clinical parameters taken at a single point in time ${ }^{5,6}$. Although this cross-sectional approach can identify which patients are at risk for future events, it cannot predict at which time the event will occur. By using a different approach, we were able to identify dynamic trends in important physiological and laboratory parameters before death ${ }^{78}$, . Using this methodology, death was used as the starting point, and the behavior of predictive variables was analyzed using a backward approach. Applying this approach we observed an accelerated decline in key parameters such as albumin, systolic blood pressure and inter-dialytic weight gain next to increases in C-reactive protein as early as 40 weeks before death ${ }^{8}$. We believe that studies of the dynamic behavior of clinical and laboratory markers before hospitalization, using the approach of our previous paper ${ }^{8}$, may aid to the development of prediction systems to timely identify patients at risk. We hypothesize that changes in important patient parameters also occur before hospitalization.

The first aim of the present analysis was to study the dynamics of hospitalization rates before death and to describe the dynamics of selected clinical and laboratory parameters before death in a large cohort of US HD patients. The second goal was to identify dynamic patterns in clinical and laboratory parameters before the first hospitalization episode. 


\section{Methods}

As indicated in the study flow chart (Figure 6.1), we conducted two separate analyses:

\section{1) Hospitalization rates before death in relation to dynamics of clinical and laboratory parameters}

For the analysis of the hospitalization rates before death, we studied all in-center HD patients treated in 51 Renal Research Institute (RRI) clinics, who died between Jan 1, 2007 and December 31, 2011. Only the patients who were dialyzed at least 24 months prior to death were included in this analysis (Figure 6.1, left). Although this time frame is to some degree arbitrary, a longer time period was chosen in order to properly assess when the change in hospital admissions happens most precipitously. The averages of hospital admission rates were computed monthly in the 24 months prior death. Hospitalizations related to kidney transplant procedures were excluded from the analysis $(\mathrm{N}=43)$. Causes of death were classified based on ICD-9 documented diagnosis into four categories: cardiovascular disease (CVD), infection, neoplasm, and other. Hospital admissions were categorized based on the primary discharge diagnosis as access related, fluid overload, CVD other than fluid-overload, infections, and other causes. Other CVD include hospitalizations for chest pain, hypo- and hypertension, cardiac arrest, atrial fibrillation and others.

Hospital admission rates were computed monthly prior to death based on number of admissions and number of patient months. Averages of patient laboratory and treatment parameters were computed per patient per month prior to death (predialysis systolic blood pressure, pre-dialysis body temperature, intradialytic change in blood pressure, inter-dialytic weight gain, effective treatment time, average saline use, hemoglobin, albumin, and neutrophil-to lymphocyte ratio (NLR).

\section{2) Dynamics in clinical and laboratory parameters before first hospitalization}

All in-center HD patients treated in the 51 RRI clinics who were hospitalized at least once between Jan 1, 2007 and Dec 31, 2011 were included in this analysis. First hospitalization per patient during period was noted and patients' laboratory and treatment parameters for the six months prior to this date of first hospitalization were obtained. Patients who had $<70$ treatments and who were transferred to a non-RRI clinic in any of the 26 weeks prior to hospitalization were excluded (Figure 6.1, right). Causes of hospitalization were divided into five groups as was done in part 1. Hospitalizations related to kidney transplantation were excluded $(\mathrm{N}=131)$. 
As a comparison group, we identified patients with vintage equal to the mean vintage of the hospitalized patients (mean=4.1 years) and computed mean of patients' laboratory and treatment parameters in a 6-months period between months 43 and 49 , i.e. the six months prior to the end of the 4.1 years. Only patients who had no hospitalization in this six month period were selected.

Laboratory results were automatically downloaded into the RRI data base from a central laboratory (Spectra Laboratories; Rockleigh, NJ). Blood pressure, body temperature, and body weight were recorded by the dialysis clinic staff. No imputation of laboratory or treatment parameters were done if patients missed their treatments-only recorded data was used. Record of hospital admission is made by the clinic staff and those are required if a patient missed at least one treatment. Both automatic and manual procedures were put in place to identify and correct data entry errors. Monthly averages of individual patient laboratory and treatment parameters were computed prior to hospitalization. Linear mixed effect models (LMM) were constructed for each treatment and laboratory parameter with months to hospitalization as a predictor and fixed and random effects accounting for inter- and intra-patient variability. Penalized cubic b-spline functions were graphed for variables that changed significantly prior to hospitalization based on linear mixed effect models slopes; 95\% confidence intervals for the spline functions are also presented to demonstrate which changes are significant ${ }^{9}$. The analyses were conducted in SPSS version 18 (IBM, Somers, NY) and SAS version 9.3 (SAS Institute, Cary, NC).

Analysis of hospitalization before death

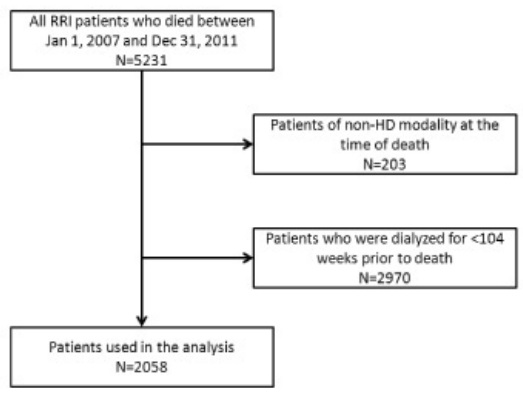

Analysis of events before hospitalization

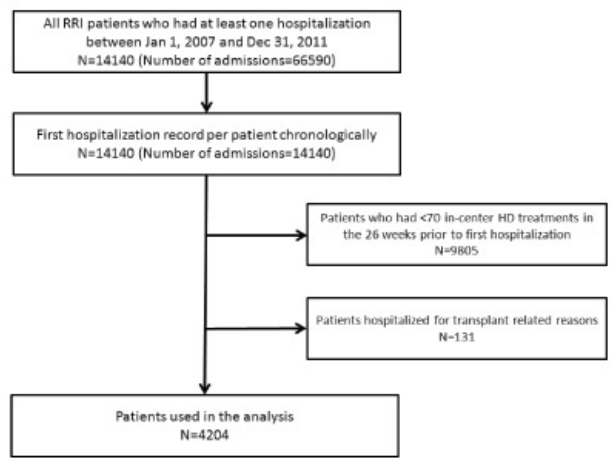

Figure 6.1 Study flowchart. 


\section{Results}

\section{Hospitalization rates before death in relation to dynamics of clinical and laboratory parameters}

We analyzed 2058 deaths: 1366 (66\%) were CVD-related, 301 (15\%) were infectionrelated, 125 (6\%) were due to neoplasms, 266 (13\%) were for other causes. Hospital admission rates increased exponentially from 0.17 admissions per patient month 24 months prior to death to 1.85 admissions per patient month in the month of death, representing an 11 times increase in hospitalization rate (Figure 6.2, left). This increase was independent of the cause of hospitalization (Figure 6.2, right) with the notable exception of vascular access interventions, which did not increase prior to death. Hospitalizations due to "other CVD" causes increased more than 16 times from 0.03 to 0.55 admissions per patient month between 24 months prior to death and the month of death. Hospitalizations due to infections, fluid overload, and other reasons increased 9 to 11 times between 24 months prior to and the month of death. The increase in number of hospital admissions was similar to patients who had 12 or more months of follow up before death (data not shown).
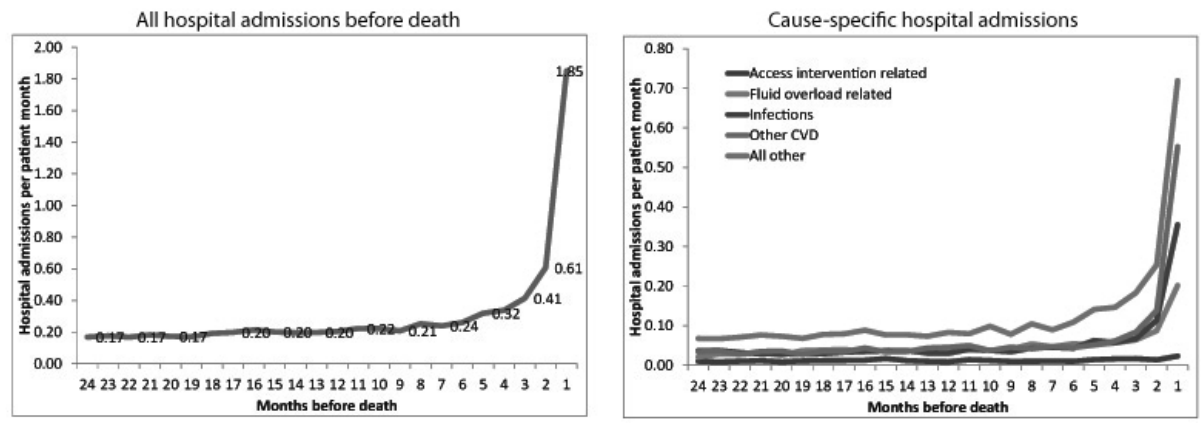

Figure 6.2 Hospital admission rates before death.

Analysis of hospital admissions by cause of death indicated comparable patterns: patients who died of CVD had the highest hospital admission rate before death (1.11 admissions per patient month), while those who died of neoplasms had the lowest number of admissions ( 0.12 admissions per patient month).

In addition to exponential increases in hospital admissions prior to death, other laboratory and patient treatment parameters showed notable patterns before death: mean albumin levels dropped from $3.8 \mathrm{~g} / \mathrm{dl}$ to $3.5 \mathrm{~g} / \mathrm{dl}$, pre-dialysis systolic blood pressure (SBP) dropped from $151 \mathrm{mmHg}$ to $137 \mathrm{mmHg}$ and NLR increased from 4.3 to 
7.0 in the 24 months before death (Figure 6.3). Patient demographics are presented in Table 6.1.

\section{Dynamics in clinical and laboratory parameters before first hospitalization}

The primary discharge diagnoses of the first hospital admission in 4204 patients were distributed as follows: access related 291 (7\%); fluid overload 467 (11\%); infections 820 (19\%); CVD unrelated to fluid overload 858 (20\%); 1768 (42\%) admissions occurred for other reasons.

Overall slopes of the treatment and laboratory parameters computed using linear mixed effects model in the six months prior to hospitalization are presented in Table 6.2. Hospitalization-cause specific slopes are not presented.

While pre-dialysis systolic blood (preSBP) did not appear to follow significant trends prior to the first hospital admissions, the intra-dialytic decline in SBP, computed as pre-HD minus post-HD SBP, became less before hospital admission. This reduction was most apparent in patients admitted because of fluid overload (Figure 6.4). Average intra-dialytic SBP decline dropped from 11.5 to $10.2 \mathrm{mmHg}$ during a 26 week period before hospitalization; the slope of the decline was significant using LMM.

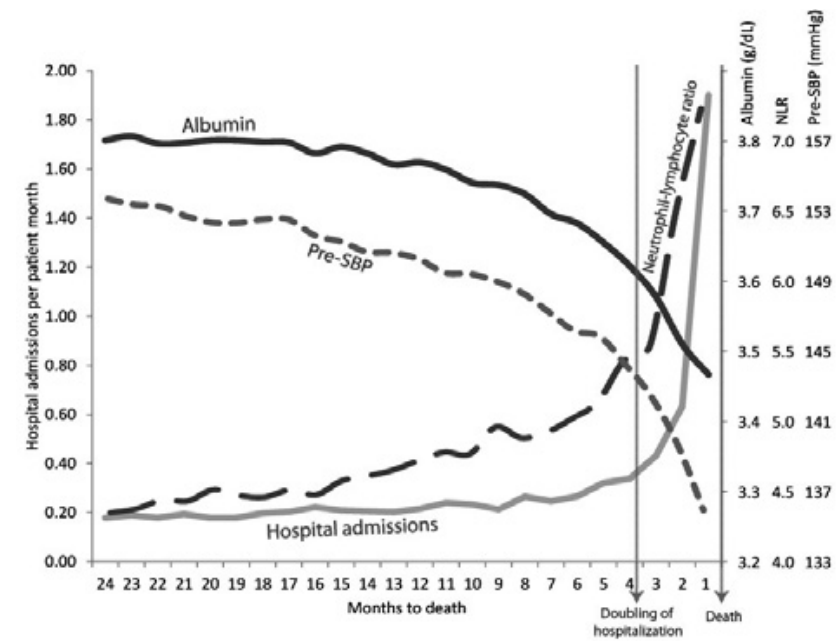

Figure 6.3 Hospital admission rate and selected clinical and laboratory parameters before death. 
Chapter 6

Table 6.1 Demographics.

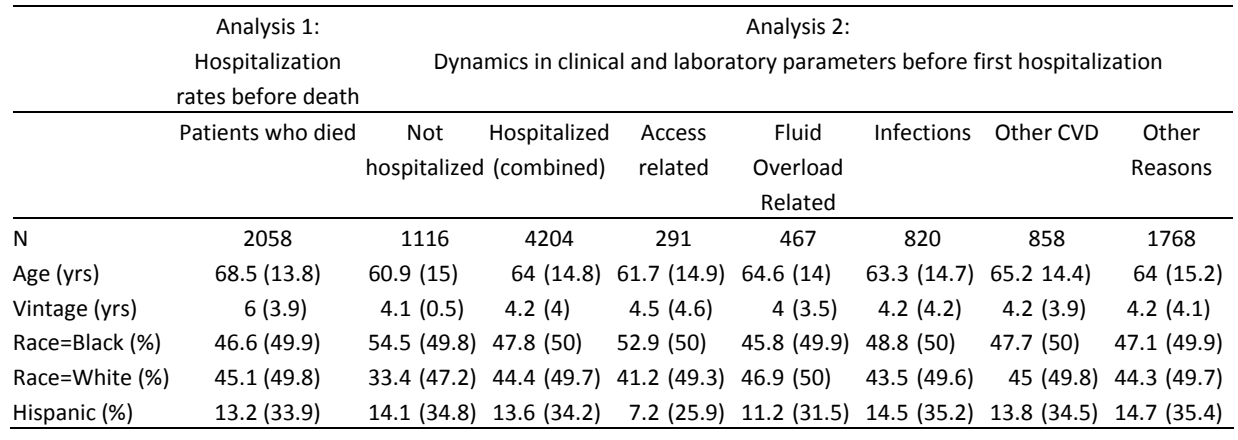

All variables are presented as means (standard deviations)

Table 6.2 Mean patient and laboratory parameters six months before and month of hospital admission and average monthly change in these parameters.

\begin{tabular}{lccc}
\hline & $\begin{array}{c}\text { Average six } \\
\text { months before } \\
\text { hospital } \\
\text { admissions }\end{array}$ & $\begin{array}{c}\text { Average in the } \\
\text { month of } \\
\text { hospital } \\
\text { admission }\end{array}$ & $\begin{array}{c}\text { LMM monthly slope (95\% Cl) } \\
\text { units/months }\end{array}$ \\
\hline Intradialytic change in systolic blood pressure $(\mathrm{mmHg})$ & $11.4(27.67)$ & $10.2(30.2)$ & $-0.09(-0.17$ to -0.01$)$ \\
Interdialytic weight gain (kg) & $2.76(1.5)$ & $2.67(1.51)$ & $-0.009(-0.013$ to -0.005$)$ \\
Neutrophil to lymphocyte ratio & $3.7(3.48)$ & $4.5(4.17)$ & $0.08(0.04$ to 0.13$)$ \\
Hgb (g/dl) & $11.87(1.43)$ & $11.65(1.48)$ & $-0.04(-0.04$ to -0.03$)$ \\
Albumin (g/dl) & $3.86(0.4)$ & $3.87(0.38)$ & $0.001(-0.001$ to 0.002$)$ \\
Treatment time (min) & $214.6(30.9)$ & $212.9(33.2)$ & $-0.19(-0.26$ to -0.12$)$ \\
Pre-dialysis systolic blood pressure (mmHg) & $149.6(26.5)$ & $149.5(27.6)$ & $0.03(-0.06$ to 0.12$)$ \\
Pre-dialysis body temperature (Celsius) & $36.48(0.43)$ & $36.48(0.46)$ & $-0.0016(-0.0027$ to -0.0005$)$ \\
Saline use per treatment (ml) & $460(266.3)$ & $467(254.7)$ & $1.43(0.79$ to 2.08$)$ \\
\hline
\end{tabular}

Average change is computed from Linear Mixed Effects Model in the six months prior to hospitalization

Inter-dialytic weight gain declined from $2.76 \mathrm{~kg}$ at 26 weeks prior to hospitalization to $2.67 \mathrm{~kg}$ in the month of hospital admission. The decline was most pronounced in the patients hospitalized for infections. The volume of saline administered during HD increased by $7 \mathrm{ml}$ from $460 \mathrm{ml}$ to $467 \mathrm{ml}$, suggesting an increased frequency of intradialytic hypotensive episodes. Effective treatment time decreased from 215 minutes six months before admission to 213 prior to hospital admission.

While pre-dialysis body temperature declined in general prior to hospitalizations, it rose from 36.5 to $36.7^{\circ} \mathrm{C}$ in the 3 weeks prior to hospital admission in patients hospitalized because of infections (data not shown). In the patients hospitalized for infections, an increase in NLR was observed from 3.5 to 5.2 in the three months prior to hospitalization. Albumin, however, decreased more gradually; the decline in albumin was not significant during six months but was significant during three months prior to admission (data not shown for three month decline). In all patients, 
hemoglobin levels declined on average from $11.9 \mathrm{~g} / \mathrm{dl}$ to $11.7 \mathrm{~g} / \mathrm{dl}$, particularly in patients hospitalized for fluid overload.

Patients with the same vintage as the mean vintage in the hospitalized patients (4.1 years) but no hospitalization between months 43 and 49 served as a control group $(\mathrm{N}=1116)$. In this cohort no notable patterns in inter-dialytic weight gain, intra-dialytic change in systolic blood pressure, NLR, and Hgb were observed (Figure 6.4). Because some patients included in the hospitalized analysis above may not have been hospitalized at vintage of 4.1 years $(\mathrm{N}=588)$ they were included in both cohorts; in a sensitivity analysis, without these patients and found similar results (data not shown). Patient demographic information for hospitalized and not hospitalized patients is presented in Table 6.1.

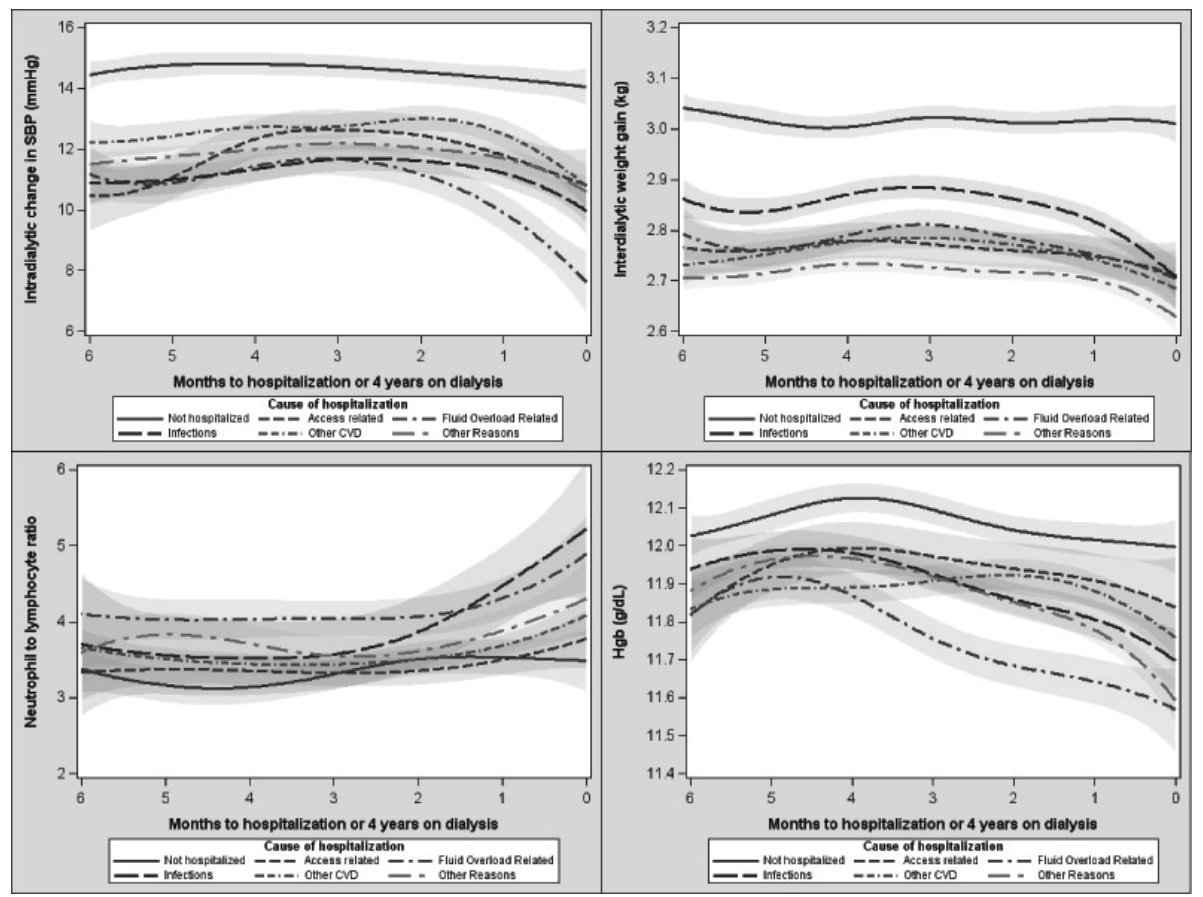

Figure 6.4 Dynamics of laboratory and treatment parameters in hospitalized patients (by cause of hospitalization) and non-hospitalized patients. 


\section{Discussion}

The outcome of our study in chronic HD patients are two novel findings: first, hospitalization rates rise sharply before death; second, distinct changes in clinical and laboratory indicators antedate the first hospitalization.

An increase in hospitalization rates before death was observed for most causes (fluid overload, infection related, CVD, and other reasons) with the sole exception of noninfectious access-related interventions. In the final 4 months of life hospitalization rates doubled every 5-6 weeks. Notably, decline in SBP and serum albumin, and increase in NLR preceded the sharp increase in hospitalization rate by almost one year $^{7}$. An important question beyond the scope of the present study is whether timely diagnostic and therapeutic interventions might prevent this increase in hospitalization rates and subsequent death within a "window of opportunity". One of the possibilities is that at the inflection point indicating the sharp upward trend of the hospitalization rate, patients are already at a stage of irreversible homeostatic breakdown and decline in functional status, in which their ability to cope with external stressors is severely decreased. This would be analogous to an advanced stage of frailty as discussed in the geriatric literature ${ }^{10}$. The presence of frailty and a loss of functional status are strong predictors of mortality in dialysis patients ${ }^{11}$.

However, recent data have suggested potentially modifiable risk factors for readmission after hospitalization. Dalrymple ${ }^{12}$ showed an increase in hospitalization for CVD causes within 90 days after an infection-related hospitalization procedure, suggesting that increased attention to cardiovascular risk factors, such as fluid overload, might prevent readmission. Chan ${ }^{2}$ showed that optimization of hemoglobin therapy and administration of vitamin D was associated with reduced risk for readmission. Whether interventions such as intensified dialysis treatment ${ }^{13,14}$, nutritional interventions ${ }^{15}$, a meticulous search for infections and cardiovascular dysfunction, optimization of fluid status, and active rehabilitation ${ }^{16}$ would be able to reduce hospitalization rates needs to be addressed in future clinical trials.

In the second part of the study we looked in greater detail into dynamic patterns of laboratory, treatment-related and clinical parameters before the first hospitalization. Apparent changes prior to the first hospitalization admissions were observed for a variety of clinical and laboratory parameters. While in general these changes were observed for all hospitalization categories, they were less apparent for access related hospitalization. Body temperature increased 1 to 2 weeks before admission in patients admitted for infections, which is consistent with clinical experience ${ }^{17}$. More remarkable were the reduction in effective treatment time, inter-dialytic weight gain, and the reduction in intra-dialytic blood pressure changes, as well as an increased 
volume of saline infusion. These changes were not only observed before infection and CVD related hospitalizations, but also for hospitalization related to fluid overload. Although conjectural, hemodynamic deterioration related to an emergent illness might reduce tolerance to HD treatment, as indicated by a shortening of effective treatment time and the need for more saline infusions. A reduction in food and fluid intake due to loss of appetite, might explain the reduction in inter-dialytic weight gain. In addition, body cell mass may decline due to the emergent illness resulting in fluid overload if dry weight is not adequately adjusted ${ }^{18}$. In turn, the presence of fluid overload combined with a lower ultrafiltration volume might explain the reduction in intra-dialytic blood pressure changes before hospitalization. These mechanisms might be mutually reinforcing, in the sense that the presence of fluid overload provides a further stimulus for cardiovascular and inflammatory events leading to hospitalization ${ }^{19}$. The latter hypothesis would fit with the higher risk for cardiovascular hospitalization following infection-related admissions observed by Dalrymple ${ }^{12}$.

With regard to the change in laboratory parameters, a decline in hemoglobin and albumin levels was observed 1-2 months before hospitalization. This analysis is more limited as in RRI dialysis facilities, these laboratory parameters were assessed only once a month. In addition, a rise in NLR was apparent for all causes of hospitalization except for access-intervention related causes. NLR, a parameter of inflammation, was higher before hospitalization in patients admitted for fluid overload as compared to the other hospitalization categories. Fluid overload itself may result in barrier breakdown and subsequent translocation of endotoxins in the gut, and also predispose to pulmonary and skin infections ${ }^{20}$. Previous studies showed a complex relation between inflammation, fluid overload and body composition in dialysis patients $^{21,22}$.

While there is no generally accepted methodology to compare trends in hospitalized and non-hospitalized "controls", we observe no notable patterns in control patients suggesting that dynamics in hospitalized patients are indeed different.

This study adds to the current body of literature by analyzing dynamic trends in hospitalization rates and by exploring clinical and laboratory parameters before hospitalization. It also might help to explain some of the seemingly paradoxical findings related to "reverse epidemiology", such as the relation between low interdialytic weight gain and mortality. To the best of our knowledge, this is the first time that dynamic trends in clinical and laboratory parameters before hospitalization have been studied in the dialysis population.

Although the absolute changes in parameters of hospitalized patients are small, it's a mean change in a very large and highly variable group of patients, which was 
statistically significant. Our aim is to recommend that clinicians should follow trends in patient parameters in addition to its absolute values, even if those changes are small. Obvious limitations of the study are its retrospective nature and the fact that only a small number of clinical and laboratory parameters were assessed. Moreover, the broad categorization of hospitalization causes may result in a loss of information. Given the structure of the RRI database, we cannot definitely distinguish elective and non-elective hospitalization. Linear mixed models can be used to further explore the statistical significance of changes before hospitalization at various time intervals; however, the time of the inflection point (the time when the slopes of the trajectories change significantly) needs to be further elucidated. Seasonality of patient parameters may play a role in patient trajectories before hospitalization and should be considered $^{23}$. Lastly, the dynamic change in parameters in the entire study population was based on aggregates of patients, not on individual trajectories. With respect to number of hospital admissions before death, we required that patients had at least 24 months of treatments before their demise; this may introduce a bias because it excludes any patients who died within $<24$ months on dialysis. As a result, we conducted a sensitivity analysis in patients with 12 month before death and found increases in number of admissions before death similar to patients who were around for 24 months prior to death.

In conclusion, we observed a sharp increase in hospitalization rates in the months before death, which was preceded by a gradual change in selected clinical and laboratory parameters within 1 year of death. Also in the period preceding the first hospitalization, a change in clinical, dialysis-related and laboratory parameters was observed. Future studies should elucidate whether dynamic risk models can be developed which aid the timely identification of patients at risk and which support daily clinical decision making. 


\section{References}

1. Foley RN, Collins AJ. End-stage renal disease in the United States: an update from the United States Renal Data System. J Am Soc Nephrol. 2007;18:2644-8.

2. Chan KE, Lazarus JM, Wingard RL, Hakim RM. Association between repeat hospitalization and early intervention in dialysis patients following hospital discharge. Kidney Int. 2009;76:331-41.

3. Daratha KB, Short RA, Corbett CF, et al. Risks of subsequent hospitalization and death in patients with kidney disease. Clin J Am Soc Nephrol. 2012;7:409-16.

4. Behl M, Sun Y, Agaba El, et al. Death during hospitalization in patients on chronic hemodialysis. Hemodial Int. 2010;14 Suppl 1:S14-21.

5. Holland DC, Lam M. Predictors of hospitalization and death among pre-dialysis patients: a retrospective cohort study. Nephrol Dial Transplant. 2000;15:650-8.

6. Rocco MV, Soucie JM, Reboussin DM, McClellan WM. Risk factors for hospital utilization in chronic dialysis patients. Southeastern Kidney Council (Network 6). J Am Soc Nephrol. 1996;7:889-96.

7. Kotanko $P$, Thijssen S, Usvyat $L$, et al. Temporal evolution of clinical parameters before death in dialysis patients: a new concept. Blood Purif. 2009;27:38-47.

8. Usvyat LA, Barth C, Bayh I, et al. Interdialytic weight gain, systolic blood pressure, serum albumin, and C-reactive protein levels change in chronic dialysis patients prior to death. Kidney Int. 2013, Epub ahead of print

9. Eilers Paul RM, Marx Brian Flexible smoothing with B-splines and penalties. Statistical Science. 1996;11:89-121.

10. Lang PO, Michel JP, Zekry D. Frailty syndrome: a transitional state in a dynamic process. Gerontology. 2009;55:539-49.

11. Johansen KL, Chertow GM, Jin C, Kutner NG. Significance of frailty among dialysis patients. J Am Soc Nephrol. 2007;18:2960-7.

12. Dalrymple LS, Mohammed SM, Mu Y, et al. Risk of cardiovascular events after infection-related hospitalizations in older patients on dialysis. Clin J Am Soc Nephrol. 2011;6:1708-13.

13. Chertow GM, Levin NW, Beck GJ, et al. In-center hemodialysis six times per week versus three times per week. N Engl J Med. 2010;363:2287-300.

14. Johansen $\mathrm{KL}$, Zhang $\mathrm{R}$, Huang $\mathrm{Y}$, et al. Survival and hospitalization among patients using nocturnal and short daily compared to conventional hemodialysis: a USRDS study. Kidney Int. 2009;76:984-90.

15. Dong J, Ikizler TA. New insights into the role of anabolic interventions in dialysis patients with protein energy wasting. Curr Opin Nephrol Hypertens. 2009;18:469-75.

16. Jassal SV, Watson D. Doc, don't procrastinate...Rehabilitate, palliate, and advocate. Am J Kidney Dis. 2010;55:209-12.

17. Leth RA, Forman BE, Kristensen B. Predicting Bloodstream Infection via Systemic Inflammatory Response Syndrome or Biochemistry. J Emerg Med. 2013;44:550-7.

18. Heersink JT, Brown CJ, Dimaria-Ghalili RA, Locher JL. Undernutrition in hospitalized older adults: patterns and correlates, outcomes, and opportunities for intervention with a focus on processes of care. J Nutr Elder. 2010;29:4-41.

19. Ronco C, Kaushik M, Valle R, Aspromonte N, Peacock WFt. Diagnosis and management of fluid overload in heart failure and cardio-renal syndrome: the "5B" approach. Semin Nephrol. 2012;32: 129-41.

20. Mclntyre CW, Harrison LE, Eldehni MT, et al. Circulating endotoxemia: a novel factor in systemic inflammation and cardiovascular disease in chronic kidney disease. Clin J Am Soc Nephrol. 2011;6: 133-41.

21. Jacobs LH, van de Kerkhof JJ, Mingels AM, et al. Inflammation, overhydration and cardiac biomarkers in haemodialysis patients: a longitudinal study. Nephrol Dial Transplant. 2010;25:243-8.

22. Demirci MS, Demirci C, Ozdogan O, et al. Relations between malnutrition-inflammationatherosclerosis and volume status. The usefulness of bioimpedance analysis in peritoneal dialysis patients. Nephrol Dial Transplant. 2011;26:1708-16.

23. Usvyat LA, Carter M, Thijssen $\mathrm{S}$, et al. Seasonal variations in mortality, clinical, and laboratory parameters in hemodialysis patients: a 5-year cohort study. Clin J Am Soc Nephrol. 2012;7:108-15. 


\section{Chapter

\section{The MONitoring Dialysis Outcomes (MONDO) initiative}

Len A Usvyat, Yosef S Haviv, Michael Etter, Jeroen P Kooman, Daniele Marcelli, Cristina Marelli, Albert Power, Ted Toffelmire, Yuedong Wang, Peter Kotanko 


\section{Chapter 7}

\section{Abstract}

Background

Systematic collection and analysis of global hemodialysis (HD) patient data may help improving patient outcomes.

Method

The MONitoring Dialysis Outcomes (MONDO) initiative comprises data from eight dialysis providers worldwide. Data are combined into one repository. Extensive procedures are employed to merge data across countries and providers.

Results

The MONDO database comprises longitudinal data of currently 128,000 HD patients from 26 countries on five continents. Here we report data from 62,345 incident HD patients. We found lower catheter rates in South East Asia and Australia; lower hemoglobin levels in South East Asia; and higher prevalence of diabetes in North America. Longitudinal analyses suggest that declining inter-dialytic weight gain and serum phosphorus and increasing neutrophil-to-lymphocyte ratio before death in all regions studied.

Conclusion

While organizationally lean and low-cost, MONDO is the largest global dialysis database initiative to date, with a particular focus on high longitudinal data density and geographical diversity. 


\section{Background}

Data and data-driven decision support systems in health care can improve patient outcomes $^{1}$. In the United States (U.S.), doctors and hospitals are required to move to computerized medical records by $2015^{2}$. With electronic medical records and health information systems becoming more widely available, not only in the U.S., more international databases are likely to sprout ${ }^{3}$. Yet, global health databases are currently scarce. While needed to address broad research questions, world-wide registries of not just cross-sectional snapshots but longitudinal data-rich patient-specific databases are lacking. World Health Organization (WHO) comes closest in having some global health status information registries, yet the WHO data is mostly limited to crosssectional descriptive information. Many countries have domestic registries of patient information that are discipline specific, particularly for chronic diseases. For examples, in Australia, the National Joint Replacement Registry collects data on patients with joint replacement; in England, United Kingdom Association of Cancer Registries collects oncology data; in U.S., Cardiac Arrest Registry is collected by Centers for Disease Control. Some limited-purpose databases are created for specific studies. For example, INTER-HEART was created as a global case-control study that seeks to understand the importance of both traditional and emerging risk factors for acute myocardial infarction. The findings will be relevant for developing health policies that can be applied to different countries and ethnic groups ${ }^{4}$.

Similar to other fields of medicine, dialysis data are generally kept in country specific registries, such as the United States Renal Data System (USRDS). Most renal registries rely on voluntary reporting and collect limited information about practice patterns ${ }^{5}$. Yet, even these databases are not linked on a global basis. In fact, global databases of dialysis patients are very limited with the notable exception of Dialysis Outcomes and Practice Patterns Study (DOPPS). DOPPS is a prospective cohort study of hemodialysis (HD), and more recently peritoneal dialysis (PD), practices based on the collection of longitudinal data for a random sample of patients and dialysis units in currently 12 countries (Australia, Belgium, Canada, France, Germany, Japan, Italy, New Zealand, Spain, Sweden, the United Kingdom, and the United States). Since 1996, data collection has yielded detailed information on more than 55,000 patients in over 600 dialysis facilities ${ }^{6}$. In fact, DOPPS proved to be one of the success stories in dialysis research, providing insights into practice pattern and their relationship to outcomes. However, DOPPS collects longitudinal data only up to 4 times a year and usually patients are not followed over many years. In contrast, electronic health records of large dialysis providers frequently contain per-treatment data and most patients are followed throughout the whole time on dialysis ${ }^{7}$. ERA-EDTA has an international renal replacement registry that collects and merges data from the various registries in Europe. These data include the patient's date of birth, gender, cause of renal failure, date of start of first renal replacement therapy (RRT), history of RRT with dates and changes of modality, treatment center, date and cause of death and information 
concerning transfer from or to other renal registries ${ }^{8}$. Ways of reporting is different in various countries and Nephro-QUEST initiative was started to standardize data collection across European countries ${ }^{9}$. Other than DOPPS, no global dialysis database exists although patients, healthcare providers, and organizations with a global outreach (e.g. KDIGO, Kidney Disease: Improving Global Outcomes) may benefit from research possible through global CKD registries ${ }^{10}$. An ideal global dialysis database should contain a wealth of longitudinal clinical data as well as static patient information, such as patient demographics. Data should be agglomerated in such a way that they can be analyzed transparently from one country to another.

The inception of the MONitoring Dialysis Outcomes (MONDO) initiative was stimulated by research from the Renal Research Institute (RRI), New York, that suggested that in chronic HD patients treated in facilities of the RRI and Fresenius Medical Care North America (FMCNA) longitudinal trends in systolic blood pressure $(\mathrm{SBP})^{11,12}$, body weight and serum albumin levels ${ }^{13}$ were associated with an increased mortality risk. Later it was shown that changes in body temperature were also associated with poorer outcomes ${ }^{14}$. Noteworthy, these dynamic changes were obvious already months before death. These observations in U.S. dialysis populations leads to an obvious question: are these observations specific for U.S. facilities or do patients in other countries demonstrate comparable longitudinal patterns? Noteworthy, HD mortality rates differ substantially between countries and facilities $^{15,16}$. In order to further explore whether or not these patterns before death exist elsewhere, we needed access to longitudinal HD patient data from around the world. This need led to the foundation of the MONitoring Dialysis Outcomes (MONDO) initiative, where a diverse group of dialysis providers agreed to facilitate analysis of individual electronic medical records (EMR). We named the consortium MONDO to demonstrate the global nature of our initiative as well as the importance of leveraging the use of world-wide data to improving dialysis patient outcomes. Data managers and analysts from all MONDO members are involved in data extraction and analysis. Statistical data analysis is conducted under the oversight of a committee encompassing statisticians and epidemiologists from the MONDO members. While it was a daunting challenge to combine data across a host of databases, MONDO members have now access to data of nearly 130,000 HD patients and close to 23 million HD treatments between 2000 and 2012. A distinctive characteristic of MONDO is that we are gathering data at a patient- and (in most instances) treatment-level. This feature allows us to analyze individual patients longitudinally. Specifically, we are collecting data on patient demographics, comorbidities, HD treatment parameters, laboratory, and adequacy parameters. We are also collecting all morbidity and mortality outcomes.

In this report we describe the MONDO initiative, the approach taken to conduct studies, delineate incident patient characteristics as well as provide sample longitudinal patterns after initiation of HD and death. 


\section{Methods}

\section{Regulatory}

Regulatory aspects can be divided into those associated with patient-specific confidentiality issues, which are usually subject to national law and compliance regulations specific to dialysis provider. All data are de-identified and do not contain protected health information such as dates of birth, patient names or addresses. Since the data comes directly from the provider specific EMR systems, all confidential patient information is removed at that time. In addition to all aspects of patient confidentiality, MONDO is cognizant of the provider-specific compliance regulations. For example, while most data from MONDO members are analyzed at RRI and the Department of Statistics and Applied Probability, University of California at Santa Barbara, our consortium welcomes members who prefer to analyze their data locally.

\section{Funding}

MONDO has a lean organizational structure and does not have a joint budget to cover expenses and overhead. Expenses are primarily related to data analysis and statistical support. Data collection, quality control, storage, and extraction are in the responsibility of each MONDO member. Unless prevented by provider-specific compliance regulations, data analysis is done at the RRI and the University of California, Santa Barbara; most of the related expenses are covered by RRI. MONDO members hold in person meetings at least usually twice a year in conjunction with international conferences such as ASN, ERA-EDTA or WCN. The travel costs are borne by each individual MONDO member.

\section{Database specifics}

MONDO contains HD patient data from eight dialysis providers world-wide with 1052 dialysis clinics on five continents; data from Africa is forthcoming. The current MONDO members are Maastricht University Hospital [Maastricht], Hadassah Medical Center [Hadassah], Imperial College Renal and Transplant Center [Imperial College], Kingston General Hospital / Queen's University [Queen's], Fresenius Asia Pacific [FMC Asia Pacific], Fresenius Medical Care Europe, Middle East, and Africa [FMC Europe], Fresenius Medical Care Latin America [Latin America], and Renal Research Institute [RRI] (Table 7.1). Each provider has an EMR system that tracks patient data and is used in the clinics. We combined the information from these EMR systems into one database. The data we collected thus far are retrospective, but we plan to update it at least every 2 years. We are currently collecting data on in-center HD patients. Since some MONDO member organizations provide PD, we plan to extend our activities to this area. Most data we collect are from January 1, 2000 onwards or the date when the HD clinic data became available. Since the MONDO members provide care to only 
a subset of the HD patients in each respective country, the percent of represented current prevalent patients varies: it is as low as about $1 \%$ in the US, and over $40 \%$ in Portugal and Argentina. Except in countries contributing only 1-2 facilities, the majority of clinics are representatively spread over the country. For example, in the US, RRI clinics are located in California, Michigan, Illinois, North Carolina, New York, and Connecticut.

Depending on the database, data elements are either entered manually in the HD clinics or automatically transferred from another source. Manually entered data are subject to rigorous internal quality control mechanisms pursuant to country-specific database regulations. In most European clinics, Maastricht, Hadassah Medical Center and all of the RRI clinics, laboratory results are directly imported electronically from the clinical laboratories. More recently, treatment parameters from FMC Europe are also automatically entered. Irrespective of entry modality, all data undergo a comparable cleaning process, where records with implausible / impossible entries are set to a null value. In some cases, patients with improbable key dates are removed.

Table 7.2 lists the data domains by MONDO member organization. Since we use existing EMR systems to collect patient data and do not require additional data entry forms, we are reliant on what is captured in each database. Hence, the information presented in Table 7.2 is to be used as a reference on what types of data is available. We collect both clinic-specific and patient specific information. Patient specific information includes: admission and discharge data, background patient information, comorbidities, vital signs recorded before, after, and during HD, dialysis prescription, in-center administered medications, laboratory data, residual renal function, vascular access, home medications, and in-center or outpatient procedures. With the exceptions of FMC Asia Pacific, FMC Latin America and Hadassah Medical Center, treatment data are gathered for each HD session. In most data bases all laboratory results are reported whenever they become available, which may be on multiple occasions in the course of a month. In contrast, FMC Asia Pacific and FMC Latin America provide laboratory data once a month. Because units of laboratory measurement may differ between countries, we convert them into consistent units (usually SI units). Differences in laboratory methods are harder to account for. Serum albumin concentration is measured by the bromocresol green (BCG) method in most databases. The bromocresol purple (BCP) method was used in Portugal and some HD clinics in Asia. To ensure comparability between the two methods, BCP measurements were transformed to BCG values ${ }^{17}$. 


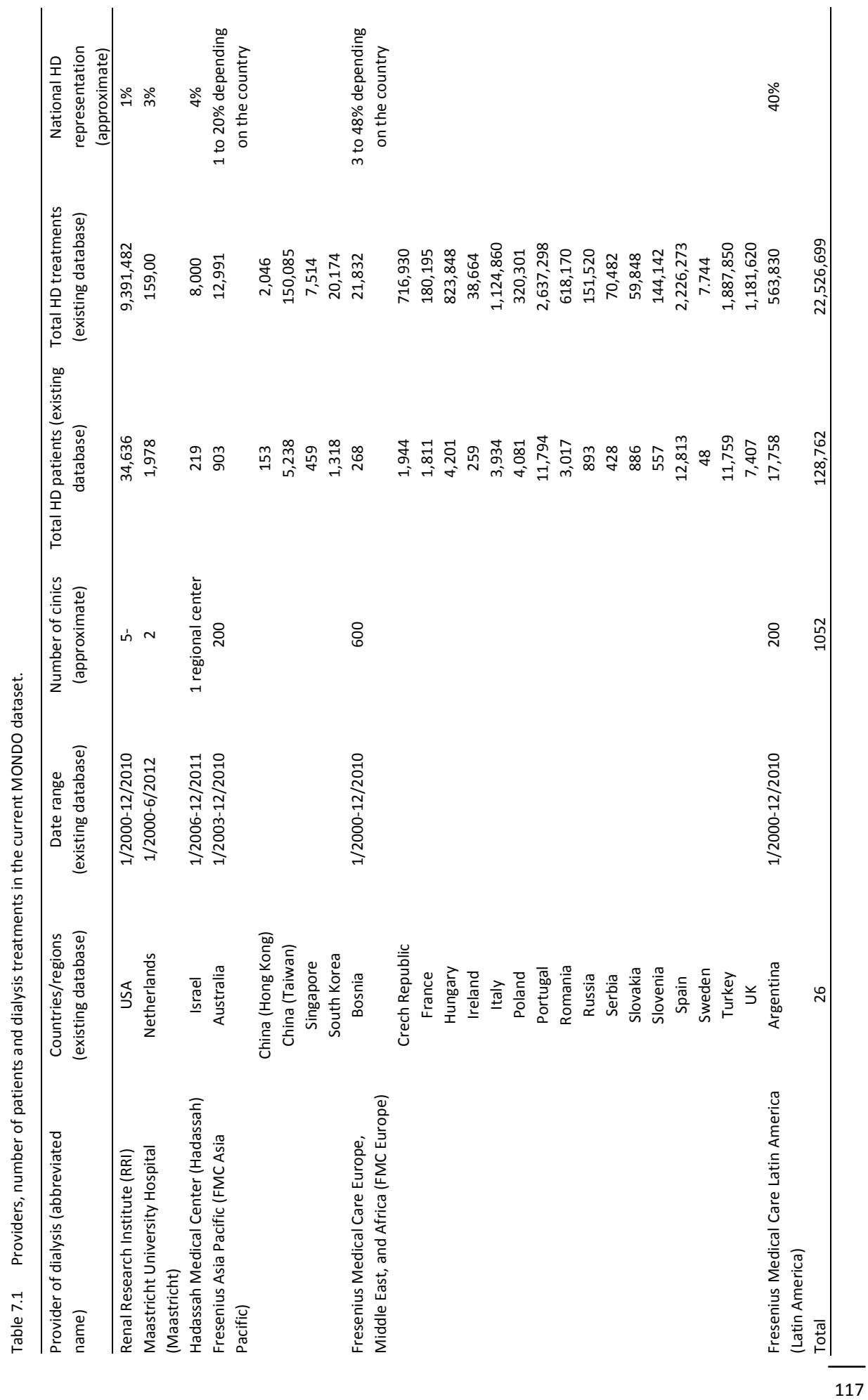




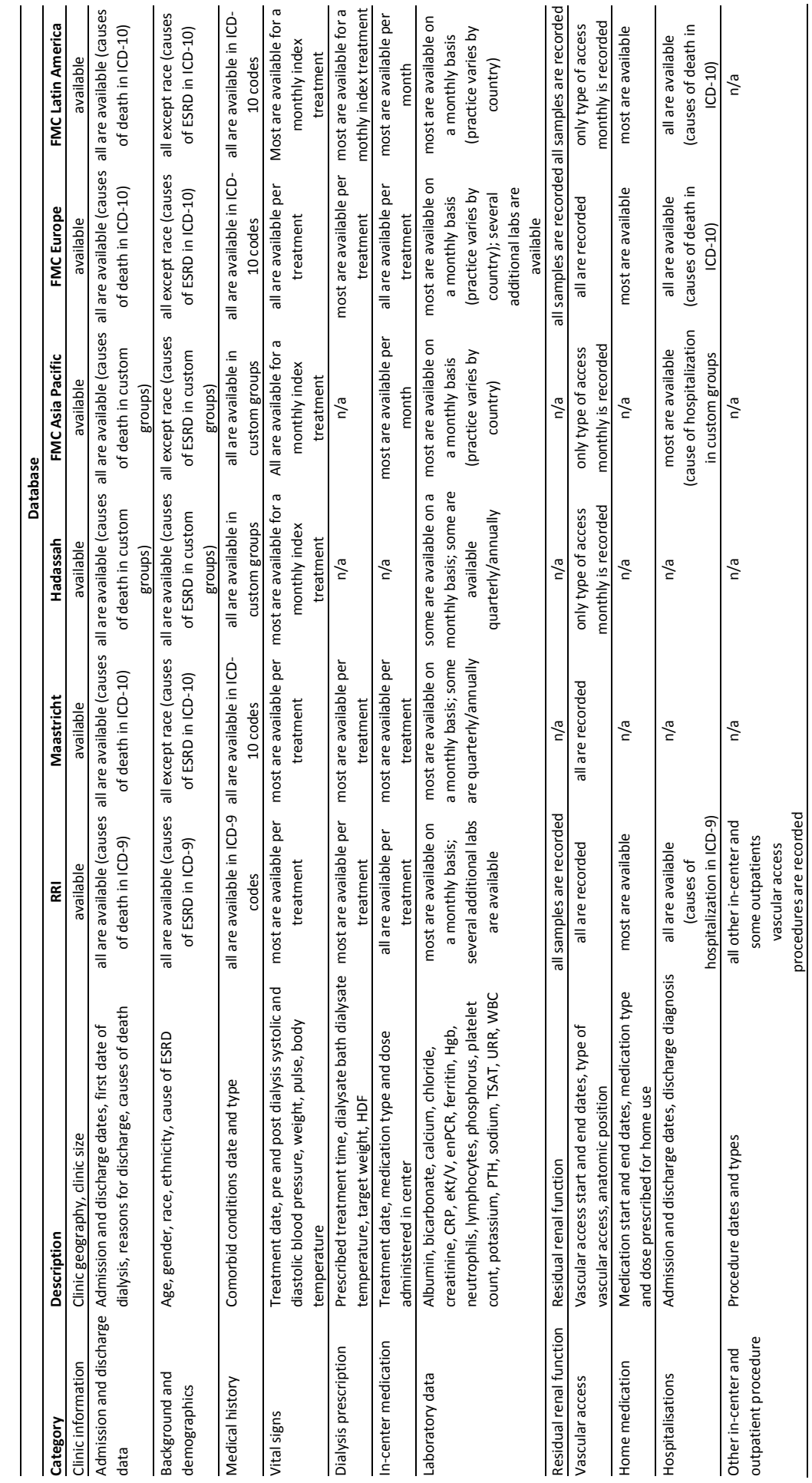


An important consideration is how to deal with the fact that some databases, by design, capture more variables than others. This is a vital decision because most analyses involve variables that exist across all databases - in essence, the "largest common number of variables." Basically, we include variables with highest research priority. However, we have the option of adding more variables in the future from the provider-specific EMR system. For example, FMC Europe collects a host of laboratory measures that are less commonly used in the U.S.. Most issues in combining the data arise from the country specific practices and regulations. Medical practices vary significantly across countries and providers, ranging from small issues such as frequently of blood pressure measurement, to specifics of drug therapy. Reimbursement systems and overarching quality goals are specific for each provider and country. In countries where patients cannot afford certain medications, e.g. cardio-protective drugs or iron supplements, reasons for lack of medication are different compared to the U.S.. Similarly, some countries allow therapies (e.g. hemodiafiltration) which may not be available in others. Many of these differences present their own layer of complexity while others create new research opportunities. Causes of ESRD, death or hospitalization, and co-morbid conditions are collected differently across databases. While some use ICD-9 or ICD-10 codes, others have developed specific descriptors. While we collect data in the original method used by each provider, we created a process that merges these schemas and allow for unified reporting and analytics. This method merges the data in such a way that has events recorded on a per-day basis, such as treatments, hospitalization records, labs results and others.

\section{Statistical methods}

In addition to measurements at dialysis initiation, the MONDO database contains a wealth of longitudinal measurements. As indicated previously, our initial aim was to investigate the existence of characteristic dynamics before death or after the start of dialysis. To that end we employ a specific analytical methodology. Here we report as examples the dynamics of inter-dialytic weight gain (IDWG), neutrophil-to-lymphocyte ratio (NLR) and serum phosphorus. We refer to the longitudinal process after the initiation of dialysis as a "forward process" since we follow the process forward in time; in contrast, we refer to the longitudinal process before death as a "backward process" since we trace the process backward in time. Understanding these dynamics is important in comprehending a more complete picture of what happens to dialysis patients over time as well as coming up with a predictive model that alerts clinicians to patients who are at increased risk of hospitalization or death. We need to estimate the mean functions of the forward and backward processes for certain populations of patients based on noisy measurements. Some of the variables are measured weekly, some are measured monthly, and some are measured at irregular times. For 
simplicity, we assume in the following discussion that monthly measurements are available (missing months are allowed).

We first describe the statistical methods for estimating the mean functions of the forward processes. We denote $\mathrm{Y}(\mathrm{t})$ as the forward process of a variable of interest $\mathrm{t}$ months after the start of dialysis from a patient in a specific population, and $T$ as the survival time of this patient after the start of dialysis. We define the partial conditional mean at month $t$ as $E(Y(t) \mid T>t)$ which represents the expectation of the forward process at month $t$ conditional on this patient survived longer than $t$ months (i.e. the patient is alive at month $t)^{18}$. To estimate $E(Y(t) \mid T>t)$, we compute average measurements of the variable of interest at month $t$ among all patients who are still alive at month $t$. These monthly averages usually have relatively large variations. To estimate the forward pattern and its trajectory, we treat these average measurements as observations and fit a quintic spline model. A quintic spline is a piecewise quintic function with a knot at each month and the fourth derivative of the function is continuous ${ }^{19}$. We use the quintic spline instead of the popular cubic spline such that the estimated trajectory is smooth. We also estimate the trajectory of the first derivative of the quintic spline function, which can be interpreted as "velocity". A similar method is used to investigate processes traced backward in time. We denote $\mathrm{Y}(\mathrm{t})$ as the backward process of a variable of interest $\mathrm{t}$ months before death, and $\mathrm{T}$ as the survival time of this patient after the start of dialysis. We define the partial conditional mean $t$ months before death as $E(Y(t) \mid T>t)$ which represents the expectation of the backward process $t$ months before death conditional on this patient survived longer than $t$ months. The condition $T>t$ avoids measurements before the start of dialysis. To estimate $E(Y(t) \mid T>t)$, we compute average measurements of the variable of interest $t$ months before death among all patients who survived longer than $t$ months. Then we fit a quintic spline model to these averages to get estimates of the mean function and its trajectory. We use the ssr function in the ASSIST R package to fit a quintic spline ${ }^{20}$. We select the smoothing parameter using the generalized maximum likelihood method and construct confidence intervals for the mean functions and their trajectories using the bootstrap method ${ }^{19}$.

To provide an overview of some characteristics of incident HD patients, we used data from 62,345 incident patients from 25 countries. Incident patients were classified as those whose in-center treatment was within 90 days of their first date of dialysis. Countries are stratified by the geographical sub-regions as classified by the United Nations $^{21}$ as North America (U.S.), South America (Argentina), South East Asia (Singapore), Eastern Asia (Hong Kong, Korea, and Taiwan), Western Asia (Israel and Turkey), Eastern Europe (Czech Republic, Hungary, Poland, Romania, Russia, and Slovakia), Southern Europe (Italy, Portugal, Serbia, Slovenia, Spain and Bosnia), Western Europe (France), Northern Europe (Sweden, Ireland, and UK), and Australia. 


\section{Results}

The MONDO consortium encompasses dialysis providers from 26 countries and approximately 1052 dialysis clinics. The precise number of clinics varies over time since dialysis providers may add or close facilities. Longitudinal data are available from over 128,000 patients and close to 22.6 million HD treatments (Table 7.1).

For this analysis, we studied 62,345 incident HD patients: 11,078 patients from North America, 14,341 from South America, 903 from Australia, 6,709 from Eastern Asia, 459 from South East Asia, 4,240 from Western Asia, 2,957 from Northern Europe, 6,662 from Eastern Europe, 14,551 from Southern Europe, and 445 from Western Europe. Mean age (in years; sorted from low to high; $(95 \% \mathrm{Cl})$ ) is in South East Asia: 56 (54.6-57.3), Eastern Asia: 58.9 (58.5-59.2), South America: 59.8 (59.6-60.1), Western Asia: 60.2 (59.8-60.7), Western Europe: 62.1 (60.5-63.8), North America: 62.3 (62-62.6), Eastern Europe: 63 (62.7-63.4), Northern Europe: 63.8 (63.1-64.4), Southern Europe: 65.5 (65.2-65.7), Australia: 65.9 (64.9-67). Percent of male patients are in Eastern Asia: 53\%, Eastern Europe: 55\%, South East Asia: 56\%, North America: 57\%, South America: 57\%, Western Asia: 57\%, Southern Europe: 61\%, Northern Europe: 63\%, Western Europe: 64\%, and Australia: 66\%.

Figure 7.1 presents selected comorbid conditions and access types at the initiation of dialysis. Figure 7.2 depicts laboratory characteristics at dialysis initiation. In addition to descriptive characteristics of incident patients, we also present examples forward and backward analyses. Figure 7.3 shows forward dynamics of IDWG, NLR, and phosphorus levels in incident HD patients by continent (North America, South America, Europe, Asia, and Australia). Figure 7.4 depicts backward dynamics of IDWG, NLR, and phosphorus levels prior to death. 


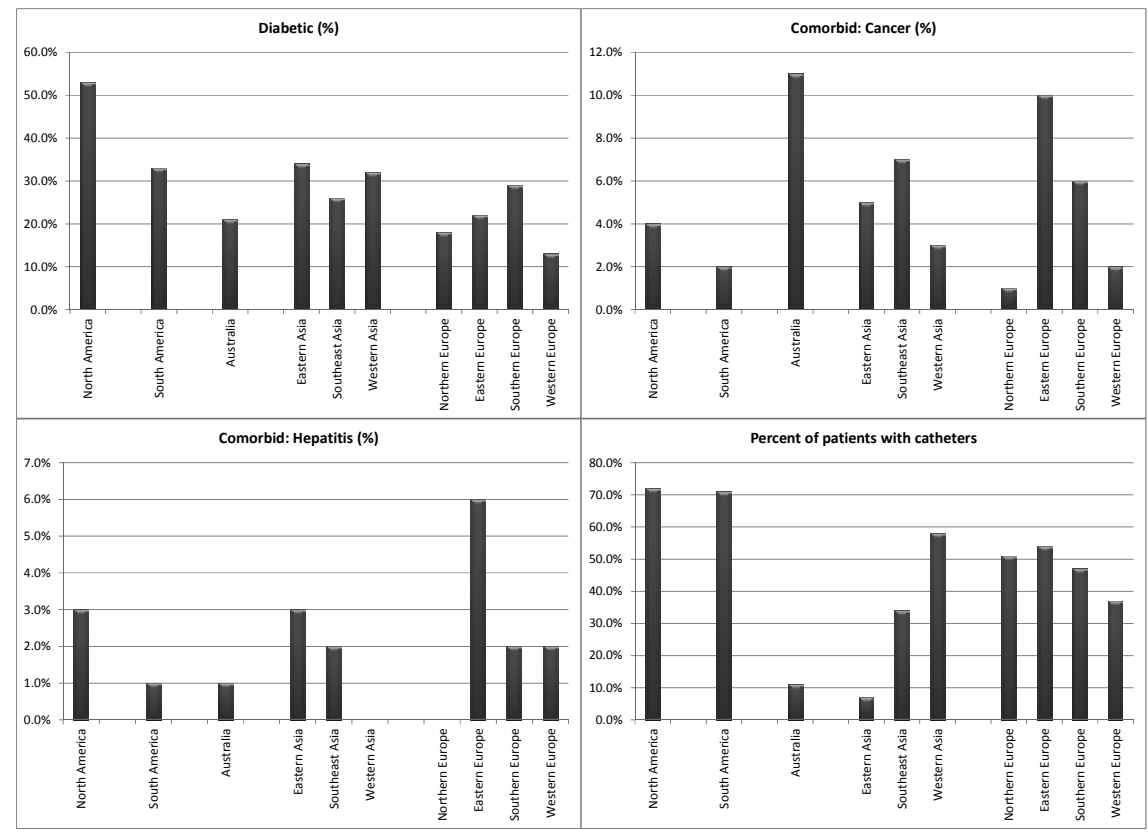

Figure 7.1 Comorbid conditions and access type at the start of dialysis by geographic region.

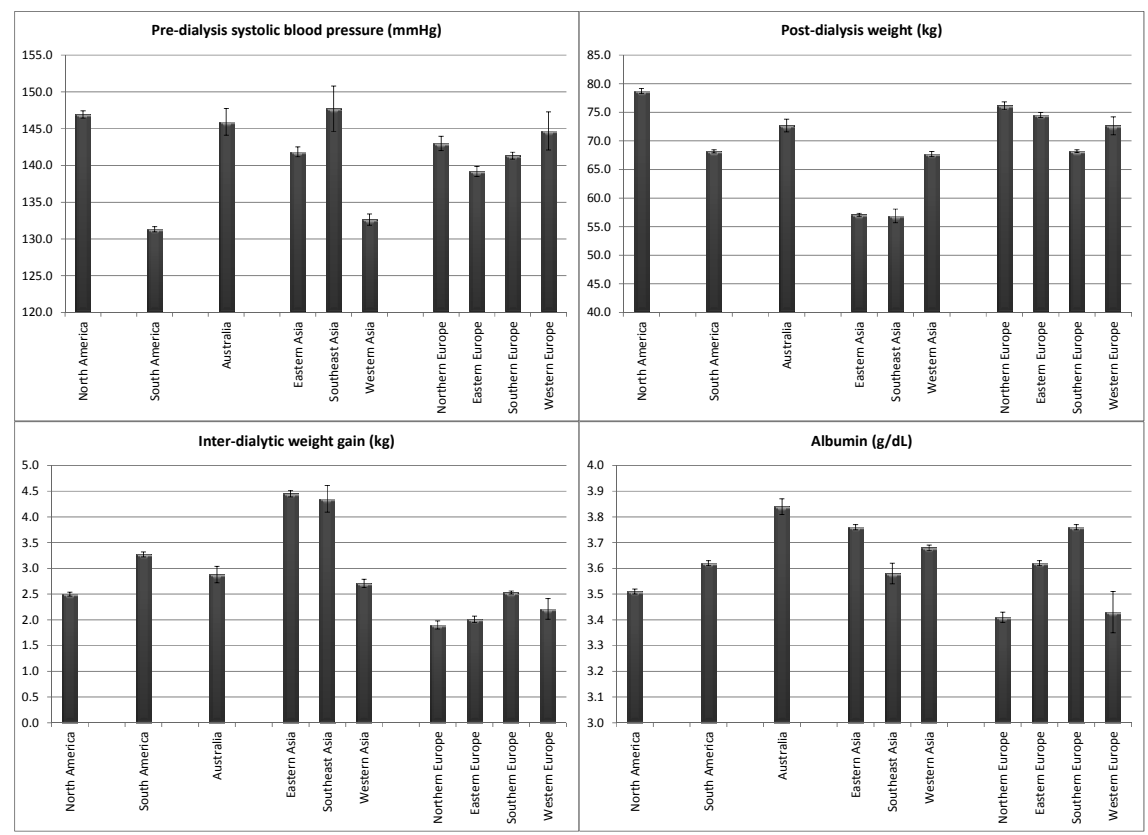




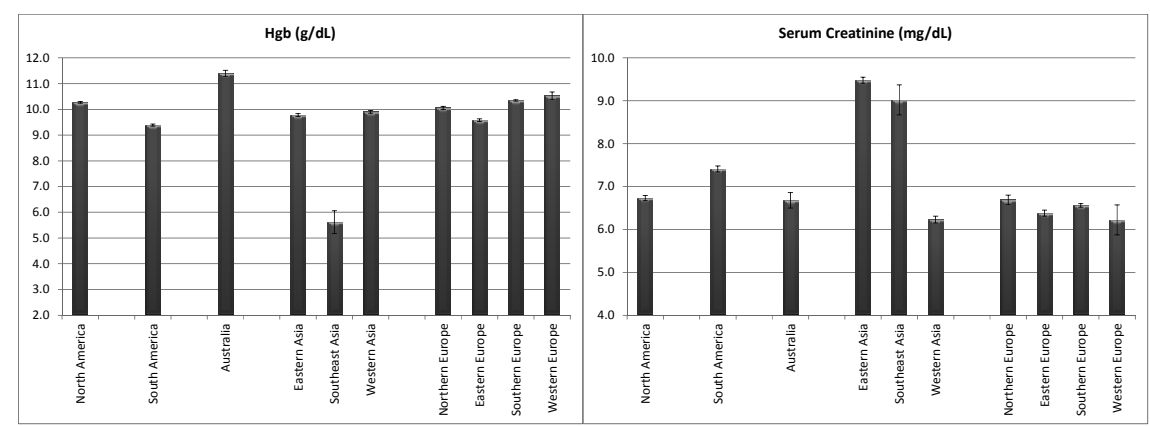

Figure 7.2 Clinical and laboratory characteristics of dialysis patients at the start of dialysis by geographic region $($ mean $\pm 95 \% \mathrm{Cl})$.
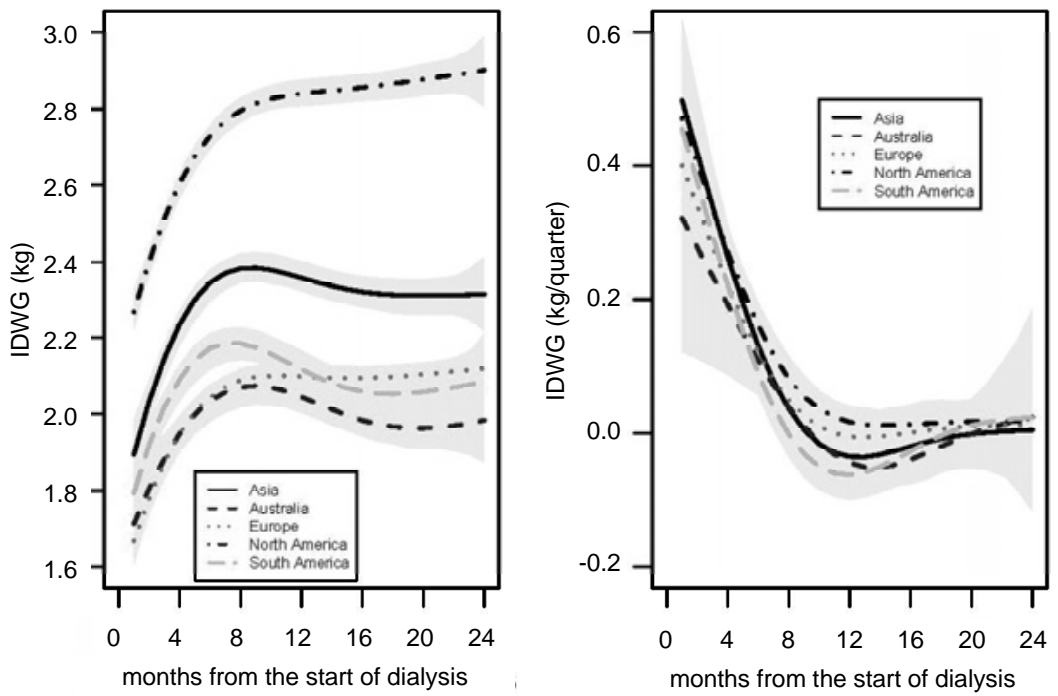

Figure 7.3a Mean inter-dialytic weight gain and 95\% confidence interval in the 24 months from the start of dialysis in incident patients who survived for more than 2 years. The left panel shows estimates of the partially conditional means and the right panel shows estimates of rate of change trajectories with $95 \%$ confidence intervals. 

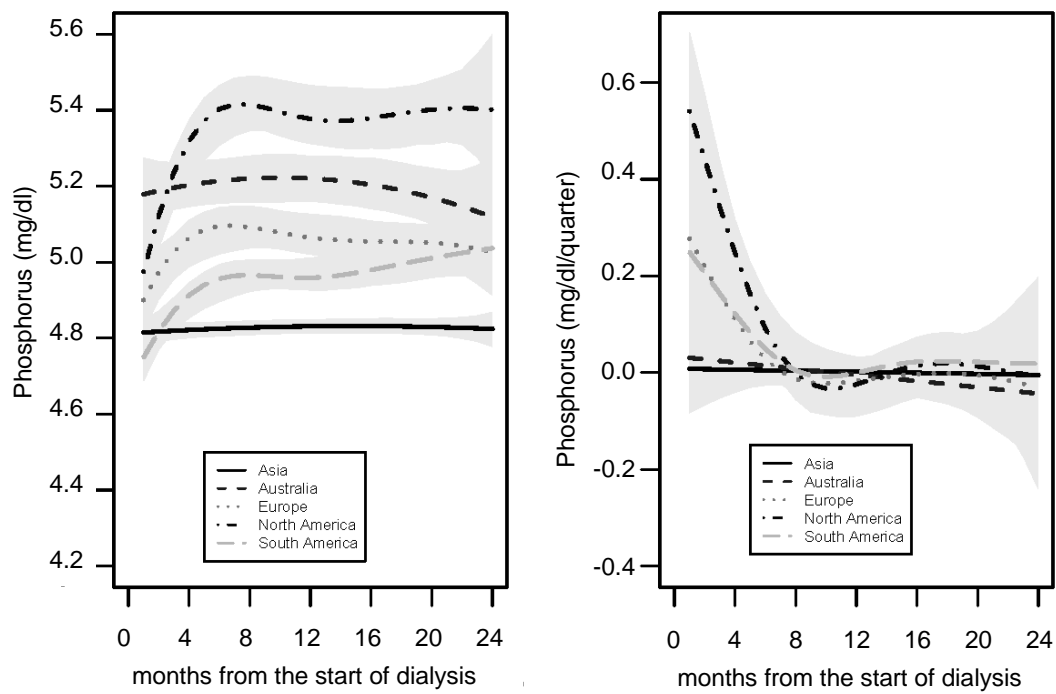

Figure 7.3b Mean serum phosphorus and 95\% confidence interval in the 24 months from the start of dialysis in incident patients who survived for more than 2 years. The left panel shows estimates of the partially conditional means and the right panel shows estimates of rate of change trajectories with $95 \%$ confidence intervals.
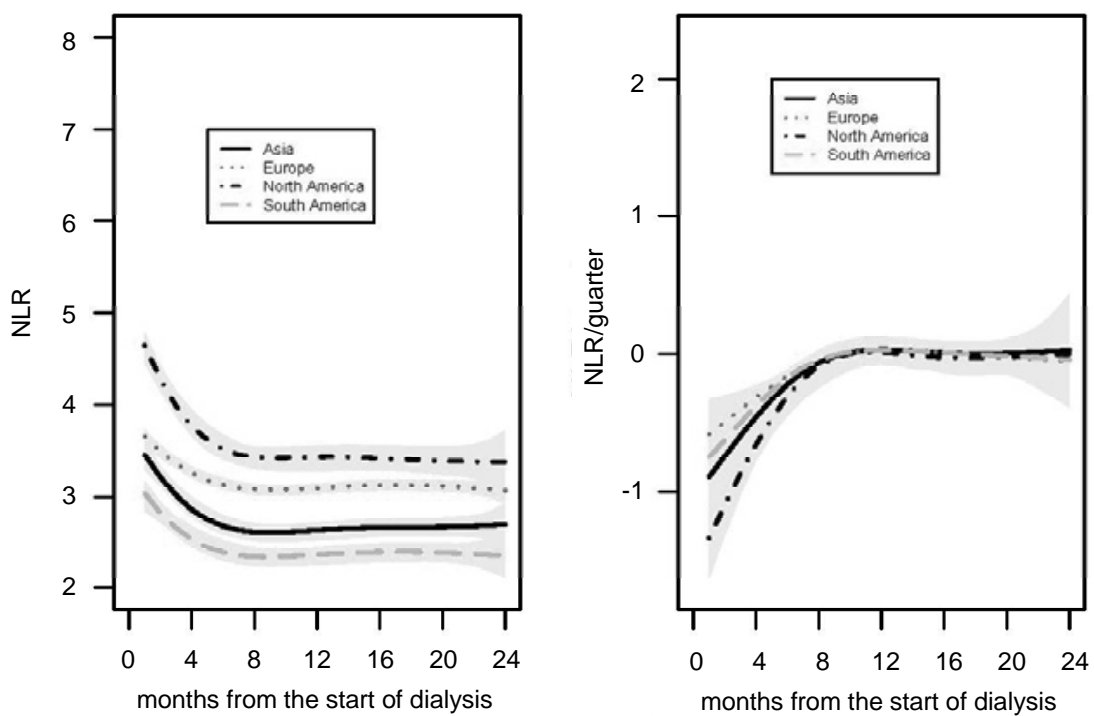

Figure 7.3c Mean neutrophil to lymphocyte ratio (NLR) and 95\% confidence interval in the 24 months from the start of dialysis in incident patients who survived for more than 2 years. The left panel shows estimates of the partially conditional means and the right panel shows estimates of rate of change trajectories with $95 \%$ confidence intervals. 

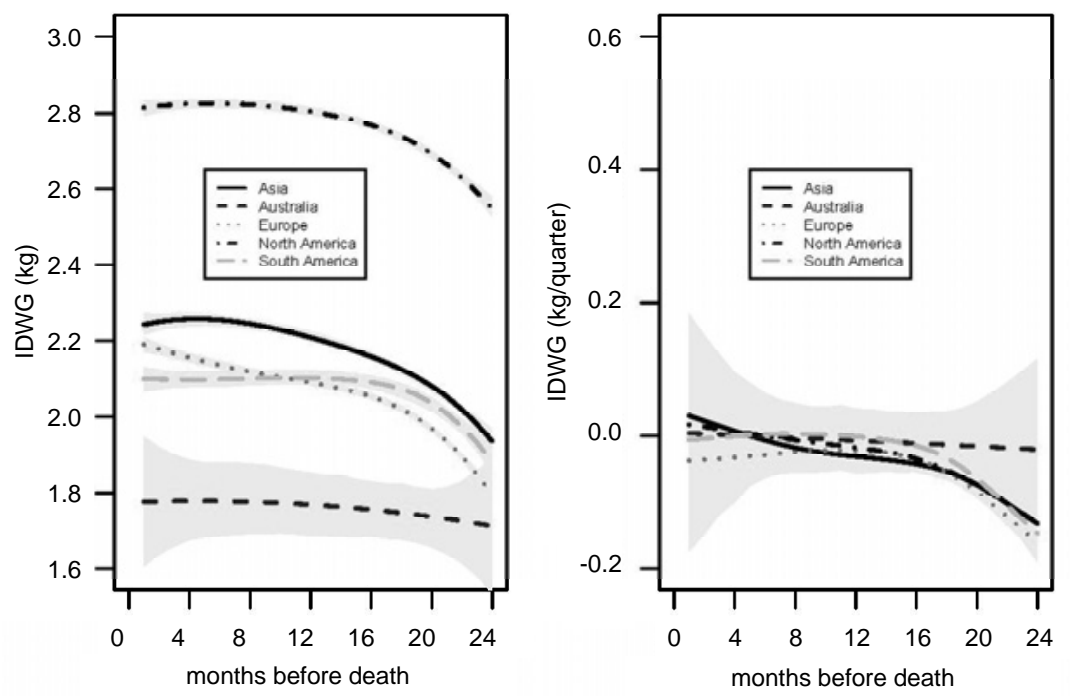

Figure 7.4a Mean inter-dialytic weight gain and 95\% confidence intervals in patients in the 24 months prior to death. The left panel shows estimates of the partially conditional means and the right panel shows estimates of rate of change trajectories with $95 \%$ confidence intervals.
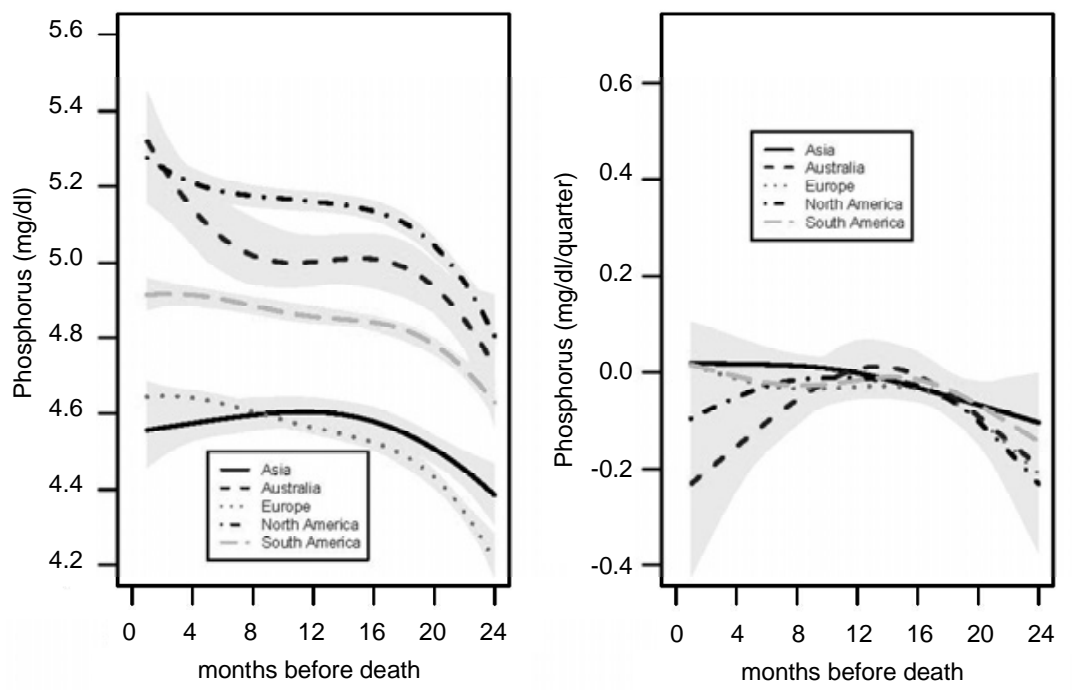

Figure 7.4b Mean serum phosphorus and 95\% confidence intervals in patients in the 24 months prior to death. The left panel shows estimates of the partially conditional means and the right panel shows estimates of rate of change trajectories with $95 \%$ confidence intervals. 

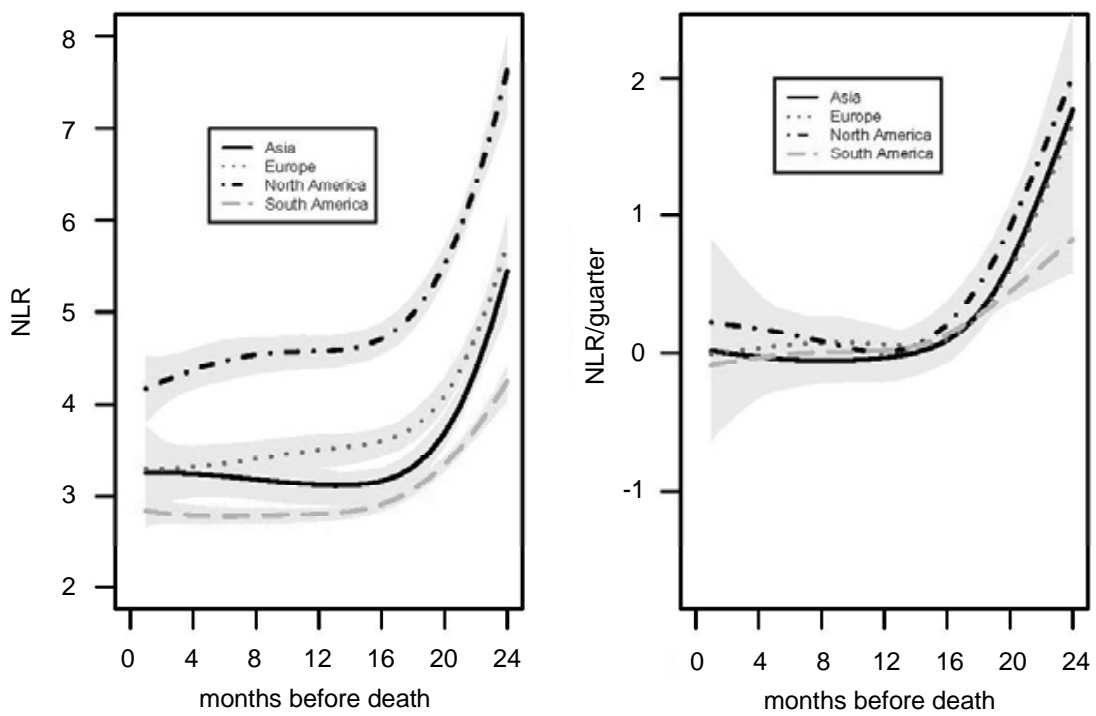

Figure 7.4c Mean neutrophil-to-lymphocyte ratio (NLR) and 95\% confidence intervals in patients in the 24 months prior to death. The left panel shows estimates of the partially conditional means and the right panel shows estimates of rate of change trajectories with $95 \%$ confidence intervals.

\section{Discussion}

In the current analysis, we present the characteristics of incident HD patients in the MONDO database. Studies have shown that certain patient characteristics such as older age, catheter vascular access, albumin $<3.5 \mathrm{~g} / \mathrm{dl}$, phosphorus $<3.5 \mathrm{mg} / \mathrm{dl}$, cancer, and congestive heart failure are associated with poor outcomes ${ }^{22}$. Here we aim to describe differences between patients and their disease status as they enter dialysis treatment. While no other studies contain data on all the geographic regions in question, DOPPS findings have shown that European and American patients tend to be older than the patients in Japan ${ }^{5}$. Similar to DOPPS findings, more than half of our patients are male ${ }^{5}$. Percent of diabetic patients is highest in North America, again consistent with DOPPS. Our data suggests that patients in Eastern Asia have the lowest percent of patients with catheters while patients in North and South America have the highest percent of patients with catheters at the initiation of dialysis. The Australian findings are consistent with the ANZDATA ${ }^{23}$. SBP appear lowest in South America and Western Asia, yet are quite similar across other regions of the world ranging between 135 and $150 \mathrm{mmHg}$. Reasons for these differences are unknown but could be related to patient comorbidities or drug therapy. Hemoglobin levels appear 
notably lower in South East Asia which maybe due to erythropoetin stimulatating agents (ESA) use in that region. Patients start with varying levels of albumin ranging from $3.4 \mathrm{~g} / \mathrm{dl}$ in Northern Europe to $3.85 \mathrm{~g} / \mathrm{dl}$ in Australia. Patients in East Asia and Southern Europe have the next highest levels of albumin which may explain their better survival rates. Serum creatinine levels appear highest in East and South East Asia. Access type, comorbidities, laboratory and clinical parameters at the initiation of dialysis are different across the world and may be associated with differences in survival, while longitudinal patterns after the start of dialysis or before death appear similar.

Prior RRI research suggested that in chronic HD patients treated in the US, a decline in $\mathrm{SBP}^{11,12}$, body weight and serum albumin levels ${ }^{13}$ was associated with an increased mortality risk. Current analysis of the MONDO data IDWG, serum phosphorus and NLR shows that significant changes in these parameters occur before death: IDWG and serum phosphorus declines while NLR increases. The similarities in these patterns are apparent irrespective of the initial levels. Declines in IDWG and serum phosphorus may suggest poorer nutrition while increases in NLR may indicate increased inflammation.

Even though MONDO started with a main objective of understanding whether patient clinical and laboratory parameters follow similar trends before death in different countries, we understand that our initiative opens a whole new realm of analyses and insights into what happens in dialysis patients worldwide. In addition to looking at other areas of dialysis research, we plan to add new variables as they become available. For example, we now collect detailed patient hospitalizations, body composition, as well as instrumental tests, such as cardiac echocardiograms and electrocardiograms. We are accommodating our database structure to allow for such a data input. MONDO also plans to expand into other renal replacement therapy modalities, in particular PD. Other fields of interest are specific patient groups, e.g. children, or patients with rare disorders, e.g. Fabry's disease and microangiopathies.

We anticipate that new members will be joining MONDO in the upcoming years. In July 2012, two dialysis providers (Imperial College Renal and Transplant Center in London, UK, and Hadassah-Hebrew University Medical Center in Jerusalem, Israel) expressed their interest in joining the initiative. Imperial College has databases of nearly 2,000 patients and Hadassah Hospital has a database of 220 patients. Data from Hadassah Hospital is already being incorporated into the current work. Existing MONDO partners are also continuously working on adding new clinics to their networks. Within less than one year, we expect to incorporate data on patients from Canada, New Zealand, Thailand, Malaysia, Philippines, South Africa, Brazil, Chile, Colombia and Venezuela. We also believe that data from United Arab Emirates are 
forthcoming (Table 7.3). MONDO's key strength is the breadth and depth of the data. With data on over 128,000 patients and 22.5 million in-center HD treatments performed in facilities from all continents, this is the largest such initiative to date. Since we rely on existing EMR system, there is no need for additional data collection forms that need to be completed in clinics. By relying on existing systems, we also have access to data elements that are not readily available. There are no overhead costs associated with entering the MONDO consortium; however, each MONDO member has to carry its costs. Diversity is a key feature of MONDO and thus we hope to bring other dialysis providers with access to patient data and scientific interest on board.

Table 7.3 Summary of existing and future countries to be included in the MONDO initiative.

\begin{tabular}{|c|c|c|c|}
\hline Providers of dialysis (abbreviated name) & $\begin{array}{l}\text { Countries/regions } \\
\text { (existing database) }\end{array}$ & $\begin{array}{l}\text { Countries (data to be } \\
\text { added in }<1 \text { year) }\end{array}$ & $\begin{array}{l}\text { Countries (data to be } \\
\text { added in }>1 \text { year) }\end{array}$ \\
\hline Renal Research Institute (RRI) & USE & & \\
\hline Maastricht University Hospital (Maastricht) & Netherlands & & \\
\hline Hadassah Medical Center (Hadassah) & Israel & & \\
\hline $\begin{array}{l}\text { Imperial College Renal and Transplant } \\
\text { Center (Imperial College) }\end{array}$ & & UK & \\
\hline $\begin{array}{l}\text { Kingston General Hospital / Queen's } \\
\text { University (Queen's) }\end{array}$ & & Canada & \\
\hline Fresenius Asia Pacific (FMC Asia Pacific) & $\begin{array}{c}\text { Australia } \\
\text { China (Hong Kong) } \\
\text { China (Taiwan) } \\
\text { Singapore } \\
\text { South Korea } \\
\end{array}$ & $\begin{array}{l}\text { New Zeeland } \\
\text { Thailand } \\
\text { Malaysia } \\
\text { Philippines }\end{array}$ & \\
\hline $\begin{array}{l}\text { Fresenius Medical Care Europe, Middle } \\
\text { East, and Africa (FMC Europe }\end{array}$ & $\begin{array}{c}\text { Bosnia } \\
\text { Czech Republic } \\
\text { France } \\
\text { Hungary } \\
\text { Ireland } \\
\text { Italy } \\
\text { Poland } \\
\text { Portugal } \\
\text { Romania } \\
\text { Russia } \\
\text { Serbia } \\
\text { Slovakia } \\
\text { Slevenia } \\
\text { Spain } \\
\text { Sweden } \\
\text { Turkey } \\
\text { UK }\end{array}$ & South Africa & UAE \\
\hline $\begin{array}{l}\text { Fresenius Medical Care Latin America (Latin } \\
\text { America) }\end{array}$ & Argentina & $\begin{array}{c}\text { Brazil } \\
\text { Chile } \\
\text { Colombia } \\
\text { Venezuela }\end{array}$ & \\
\hline Total & 26 & 11 & 1 \\
\hline
\end{tabular}


One obvious shortcoming of our initiative is the fact that MONDO members do not necessarily provide facilities representative of countries or regions. This deficiency is mitigated to some extent by the large number of patients and facilities enrolled. We also realize that while we have different providers in the consortium, a large fraction of our patients come from the global network of FMC. Another limitation is the overrepresentation of patients from Europe and Latin America. We try to address this issue by analyzing the data for each region separately. Unfortunately, Japan and mainland China are currently not represented. Data from Africa is currently not available, because substantial renal replacement programs are lacking in many African countries; we hope however, that initiatives like MONDO can highlight the need for advancement of renal replacement therapies in that region.

\section{Conclusion}

While organizationally lean and low-cost, MONDO is the largest global dialysis database initiative to date, with a particular focus on high longitudinal data density and geographical diversity. Longitudinal data are key to understanding pathophysiology and to successful decision making in medicine. With electronic health records becoming more available world-wide, more databases are like to appear. Our initiative is one such endeavor aimed to joining databases of multiple dialysis providers and academic institutions around the globe. Yet, we also appreciate that MONDO represents only a small fraction of HD patients worldwide. We expect MONDO to grow in the years to come, mainly driven by the realization that many areas of research benefit from a global view. 


\section{References}

1. Kawamoto K, Houlihan CA, Balas EA, Lobach DF. Improving clinical practice using clinical decision support systems: a systematic review of trials to identify features critical to success. BMJ. 2005;330:765.

2. Obama administration calls for electronic health records by 2015 , in USA Today.

3. Krishnan M, Wilfehrt HM, Lacson E Jr. In Data We Trust: The Role and Utility of Dialysis Provider Databases in the Policy Process. Clin J Am Soc Nephrol. 2012;7:1891-6.

4. Ounpuu S, Negassa A, Yusuf S. INTER-HEART: A global study of risk factors for acute myocardial infarction. Am Heart J. 2001;141:711-21.

5. Young E. The Dialysis Outcomes and Practice Patterns Study (DOPPS): An international hemodialysis study. Kidney Int. 2000;57:74-81.

6. Pisoni RL, Gillespie BW, Dickinson DM, Chen K, Kutner MH, Wolfe RA. The Dialysis Outcomes and Practice Patterns Study (DOPPS): design, data elements, and methodology. Am J Kidney Dis. 2004;44(5 Suppl 2):7-15.

7. Goodkin DA, Mapes DL, Held PJ, The dialysis outcomes and practice patterns study (DOPPS): how can we improve the care of hemodialysis patients? Semin Dial. 2001;14:157-9.

8. ERA-EDTA Registry. [cited; Available from: http://www.era-edta-reg.org/index.jsp?p=7.

9. Jager KJ, Zoccali C. Clinical databases and the QUEST initiative. Blood Purif. 2007;25:219-20.

10. Eckardt KU, Kasiske BL. Kidney disease: improving global outcomes. Nat Rev Nephrol. 2009;5:650-7.

11. Li Z, Lacson E Jr, Lowrie EG, Ofsthun NJ, Kuhlmann MK, Lazarus JM, Levin NW. The epidemiology of systolic blood pressure and death risk in hemodialysis patients. Am J Kidney Dis. 2006;48:606-15.

12. Raimann JG, Usvyat LA, Thijssen S, Kotanko P, Rogus J, Lacson E Jr, Levin NW. Blood pressure stability in hemodialysis patients confers a survival advantage: results from a large retrospective cohort study. Kidney Int. 2012;81:548-58.

13. Kotanko P, Thijssen S, Usvyat L, Tashman A, Kruse A, Huber C, Levin NW. Temporal evolution of clinical parameters before death in dialysis patients: a new concept. Blood Purif. 2009;27:38-47.

14. Usvyat LA, Raimann JG, Carter M, van der Sande FM, Kooman JP, Kotanko P, Levin NW. Relation between trends in body temperature and outcome in incident hemodialysis patients. Nephrol Dial Transplant. 2012;27:3255-63.

15. McClellan WM, Flanders WD, Gutman RA. Variable mortality rates among dialysis treatment centers. Ann Intern Med. 1992;117:332-6.

16. Held PJ, Brunner F, Odaka M, García JR, Port FK, Gaylin DS. Five-year survival for end-stage renal disease patients in the United States, Europe, and Japan, 1982 to 1987. Am J Kidney Dis. 1990;15: 451-7.

17. Clase CM, St Pierre MW, Churchill DN. Conversion between bromcresol green- and bromcresol purple-measured albumin in renal disease. Nephrol Dial Transplant. 2001;16:1925-9.

18. Kurland BF, Heagerty PJ. Directly parameterized regression conditioning on being alive: analysis of longitudinal data truncated by deaths. Biostatistics. 2005;6:241-58.

19. Wang Y. Smoothing Splines: Methods and Applications. 2011, New York: Champman and Hall.

20. RDC, T., R: A language and environment for statistical computing. R Foundation for Statistical Computing. 2009, Vienna: Austria.

21. United Nations Statistics Division. [cited 2012 8/20/2012]; Available from: http://unstats.un.org/ unsd/methods/m49/m49regin.htm.

22. Bradbury BD, Fissell RB, Albert JM, Anthony MS, Critchlow CW, Pisoni RL, Port FK, Gillespie BW. Predictors of early mortality among incident US hemodialysis patients in the Dialysis Outcomes and Practice Patterns Study (DOPPS). Clin J Am Soc Nephrol. 2007;2:89-99.

23. Moist LM, Chang SH, Polkinghorne KR, McDonald SP; Australia and New Zealand Dialysis and Transplant Registry (ANZDATA). Trends in hemodialysis vascular access from the Australia and New Zealand Dialysis and Transplant Registry (ANZDATA) 2000 to 2005. Am J Kidney Dis. 2007;50:612-21. 


\section{Chapter 8}

Dynamics of inter-dialytic weight gain, systolic blood pressure, serum albumin and C-reactive protein levels in chronic dialysis patients before death

Len Usvyat, Claudia Barth, Inga Bayh, Michael Etter, Gero von Gersdorff, Aileen Grassmann, Adrian Guinsburg, Maggie Lam, Daniele Marcelli, Cristina Marelli, Laura Scatizzi, Mathias Schaller, Adam Tashman, Ted Toffelmire, Stephan Thijssen, Jeroen Kooman, Frank van der Sande, Nathan W Levin, Yuedong Wang, Peter Kotanko 


\section{Abstract}

Reports from a United States (U.S.) cohort of chronic hemodialysis (HD) patients suggested that weight loss, decline in pre-dialysis systolic blood pressure (SBP) and in serum albumin levels may precede death; however, no comparative studies have yet been reported in populations from other countries. We analyzed the dynamics of interdialytic weight gain (IDWG), SBP and serum albumin levels in HD cohorts from Asia ( $N=3,593,5$ countries), Europe ( $N=35,146 ; 18$ countries), South America ( $N=8,649,1$ country), and North America $(\mathrm{N}=4,742,1$ country). In surviving prevalent patients these variables appeared to have notably different dynamics than in patients who died. While in all populations IDWG, SBP, and serum albumin levels were stable in surviving patients, these indicators declined starting more than a year before death, with dynamics being similar irrespective of gender and geographic region. In European patients, C-reactive protein (CRP) levels were available on a routine basis. While CRP levels were low and stable in surviving patients, they rose sharply before death. These findings indicate that relevant fundamental biological processes start many months before death in the majority of chronic HD patients. Elucidating these dynamics longitudinally may help to identify patients at risk and aid the development of an "alert system" to initiate timely interventions to improve outcomes. 


\section{Introduction}

Clinical experience shows that chronic hemodialysis (HD) patients expected to die within weeks share certain characteristics, such as poor nutrition and weight loss, increased inflammation, and worsening of cardiovascular status. However, more subtle changes in other clinical and laboratory parameters might occur before significant clinical deterioration becomes apparent. Various risk factors have emerged that have significant value in predicting the greatly increased mortality of dialysis patients. However, most of the studies have focused on single point in time measurements, whereas the longitudinal trend of surrogate outcome parameters and the dynamic evolution before death have not been widely studied ${ }^{1}$. Insight into temporal trends of surrogate outcome indicators are relevant both from a pathophysiological point of view and for the future development of predictive models, which might aid the clinician in timely identifying patients at risk for adverse outcomes.

Recent reports from Renal Research Institute (RRI) suggested that in chronic HD patients from the United States (U.S.), a decline in $\mathrm{SBP}^{2,3}$, body weight and serum albumin levels ${ }^{4}$ were associated with an increased mortality risk. It is noteworthy that these dynamic changes were already apparent months before death and qualitatively independent of age, gender, and race. These observations led to the hypothesis that fundamental biological processes may be operative in the majority of chronic HD patients weeks and even months before death, notwithstanding race and gender. However, the observed patterns of clinical and laboratory parameters before death may be associated with specific dialysis practices or environmental factors. Since RRI clinics follow similar dialysis procedures, the relatively homogeneous dialysis practice pattern renders the testing of this hypothesis difficult. Therefore MONDO (MONitoring Dialysis Outcomes), a multi-national consortium of HD databases encompassing patient-level data, was founded to further study this aspect. MONDO allows us to combine HD dialysis databases containing patient-level data from Asia, North and South America, and Europe. Because of its outreach, MONDO encompasses patients of different national, racial, and ethnic backgrounds. Currently, the MONDO database contains longitudinal patient-level measurements of key variables from more than 145,000 patients in 25 countries over a time period of up to 11 years. The MONDO database allowed us to test the hypothesis that the temporal evolution of certain clinical and laboratory indicators over several months before death is comparable amongst the majority of chronic HD patients, independent of gender and region of the world. We chose to study the temporal evolution of indicators of the nutritional, cardiovascular, and inflammatory domains, namely inter-dialytic weight gain (IDWG), systolic blood pressure (SBP), serum albumin, and C-reactive protein (CRP). 


\section{Results}

145,317 HD patients from 25 countries received at least one in-center HD treatment between 1/2000 and 12/2010. Of this number, 41,903 patients died (Europe: 30,539 deaths (18 countries); Asia: 1,809 deaths (5 countries); South America: 6298 deaths; North America: 3257 deaths). 10,277 incident patients survived $\geq 4$ years on HD (Europe: 4607; Asia: 1,784; South America: 2,351; North America: 1,485). Figure 8.1 presents the flowchart of the study design. Baseline characteristics of patients who died and incident patients who survived for $\geq 4$ years are presented in Table 8.1.

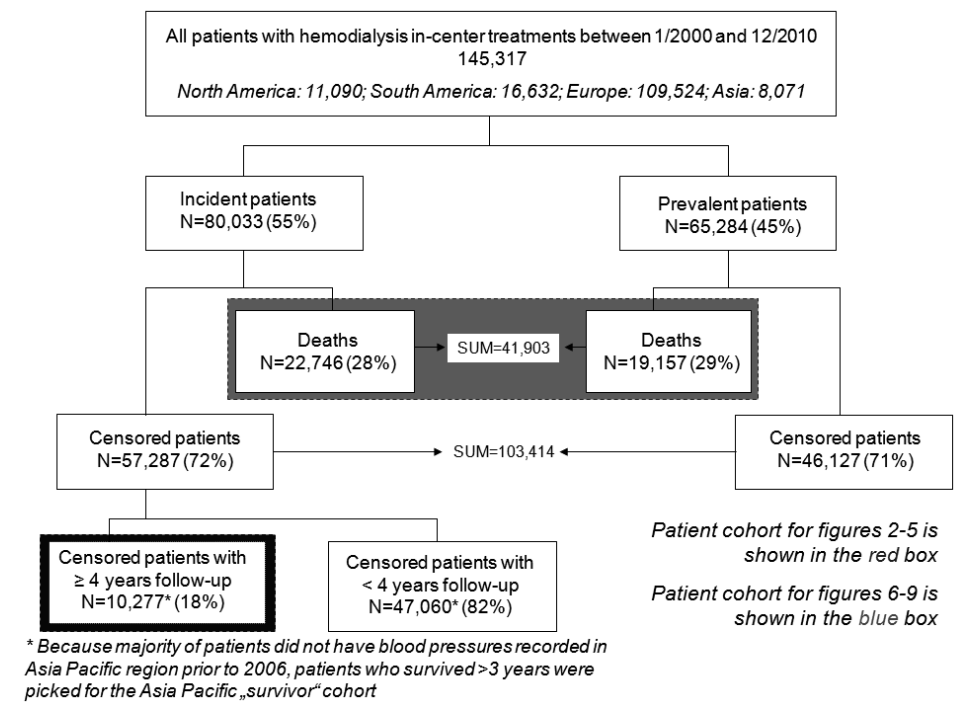

Figure 8.1 Patient flowchart.

Nearly all patients from Europe, South and North America were prescribed to dialyze thrice weekly. More than $85 \%$ of patients were dialyzed 3x weekly in Singapore and Taiwan, while $>70 \%$ were dialyzed $2 x$ weekly in Hong Kong. Dialyzer reuse is a standard practice in South America, most clinics in Singapore and in Hong Kong. All other countries use single-use dialyzers on $>90 \%$ of the treatments.

In all regions studied, serum albumin levels dropped between 0.33 (Asia) and 0.52 (North America) g/dl in the 2 years preceding death in female patients (Figure 8.2a, left). The rate of serum albumin decline accelerated before death and was comparable in Europe, South and North America, and less pronounced in Asia [Figure $8.2 \mathrm{a}$, right]. The results were materially identical in male patients (Figure 8.2b] and comparable irrespective of diabetes status (data not shown). 


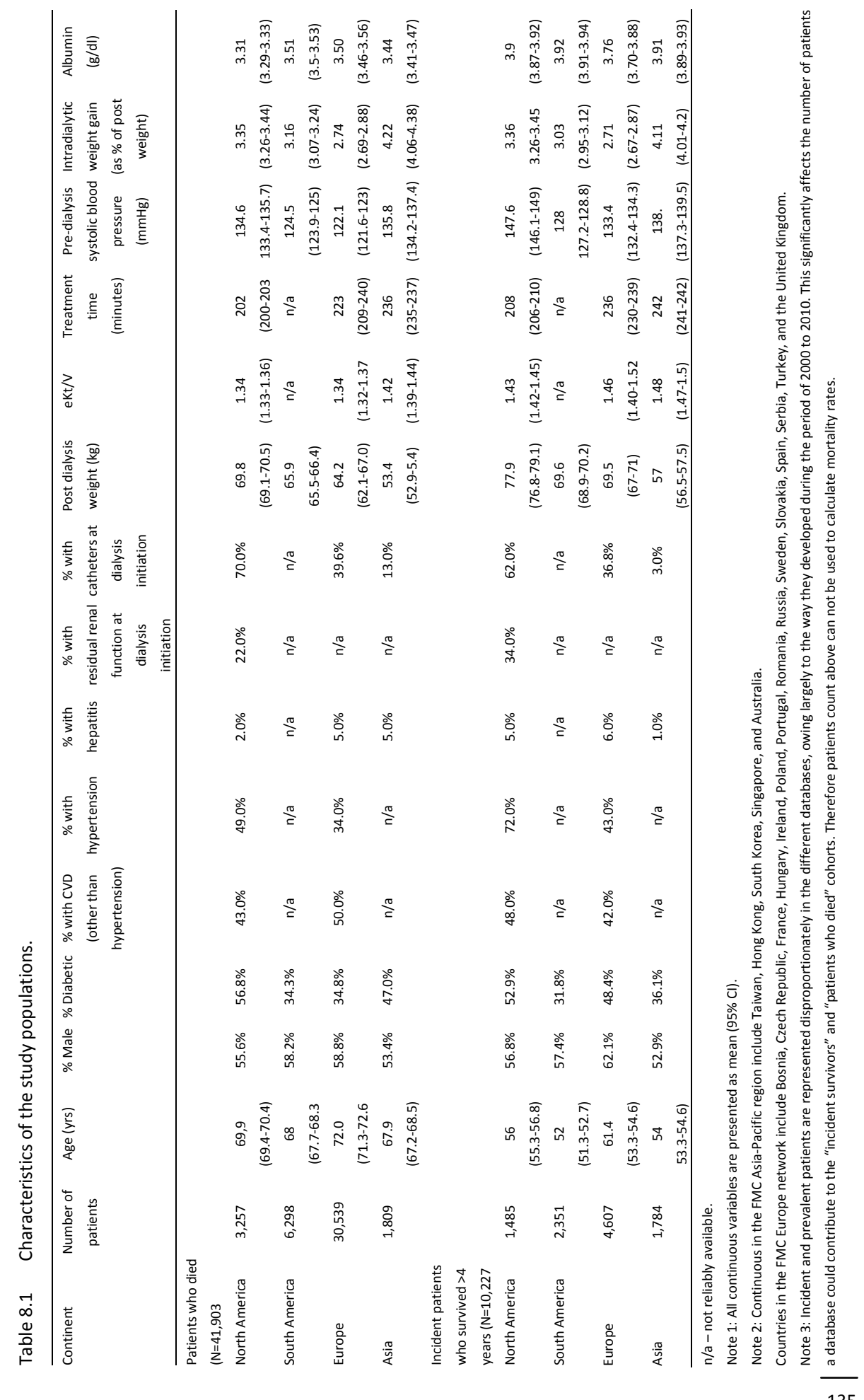



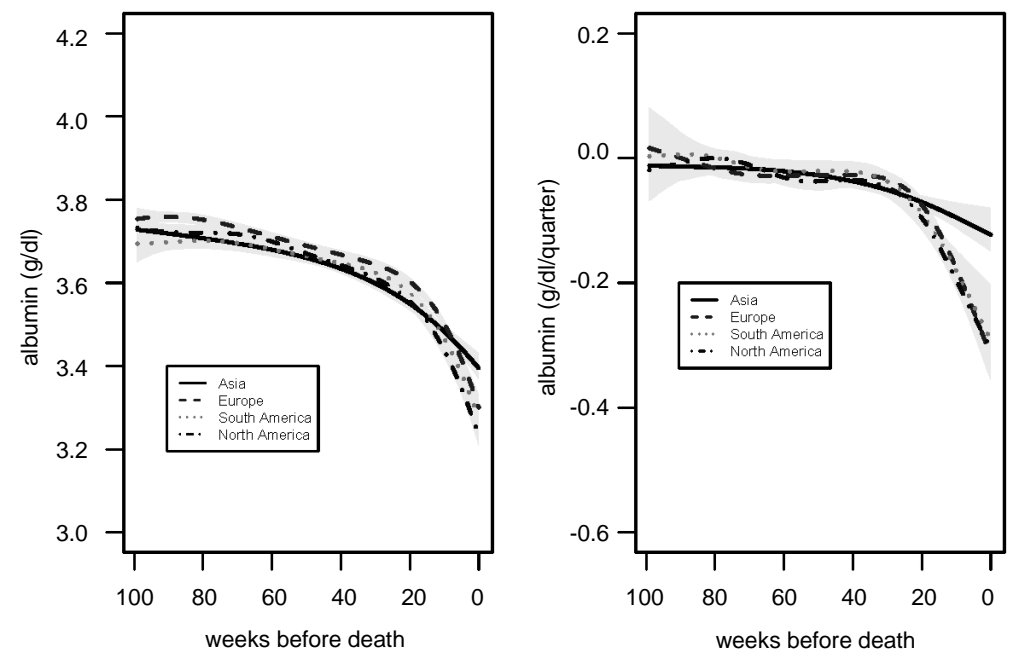

Figure 8.2a Mean serum albumin and 95\% confidence interval in female patients in the 104 weeks prior to death. The left panels show estimates of the partially conditional means with $95 \%$ confidence intervals, the right panels show estimates of rate of change trajectories with $95 \%$ confidence intervals.
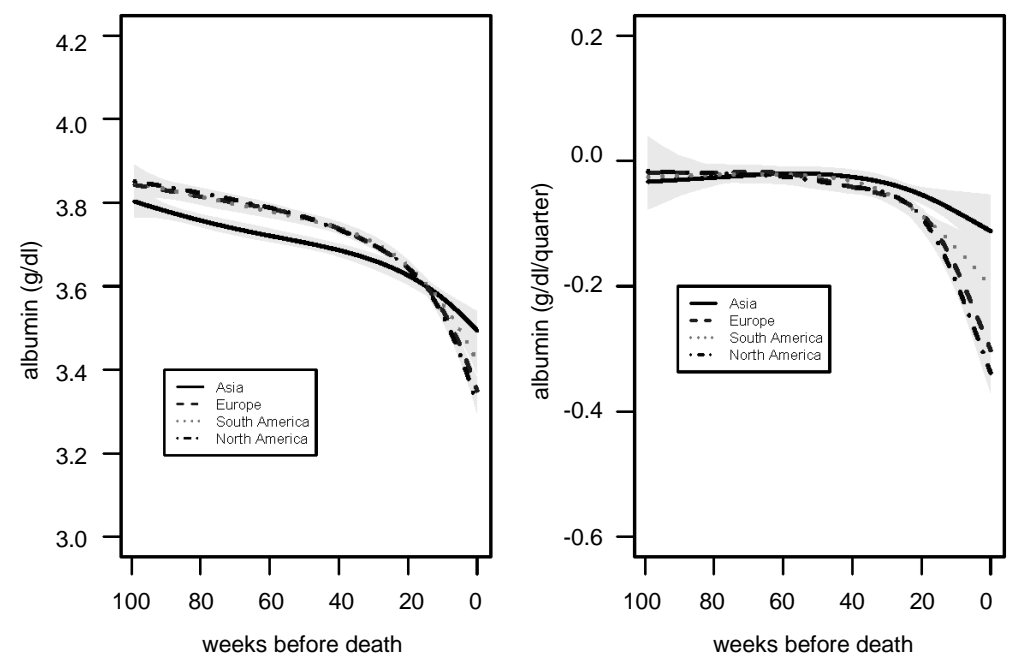

Figure 8.2b Mean serum albumin and 95\% confidence interval in male patients in the 104 weeks prior to death. 
While in Europe, North and South America, IDWG declined significantly before death, the IDWG changes in Asia were either flat or non-significant with wide confidence intervals (Figures 8.3a and 8.3b).
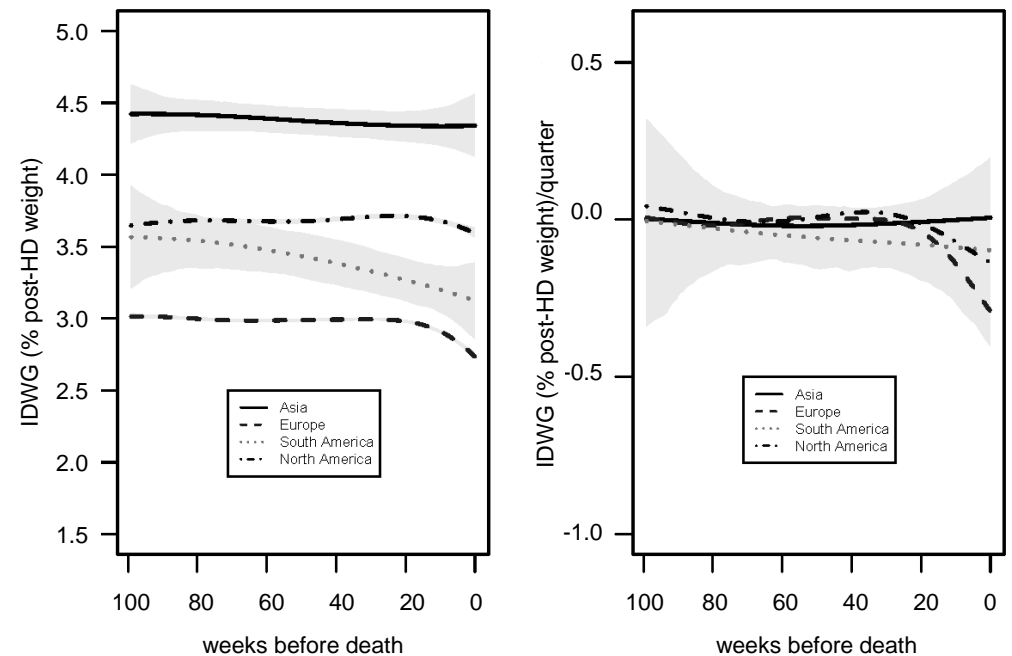

Figure 8.3a Mean IDWG (expressed as \% of post-HD weight) and 95\% confidence interval in female patients in the 104 weeks prior to death. The left panels show estimates of the partially conditional means with $95 \%$ confidence intervals, the right panels show estimates of rate of change.
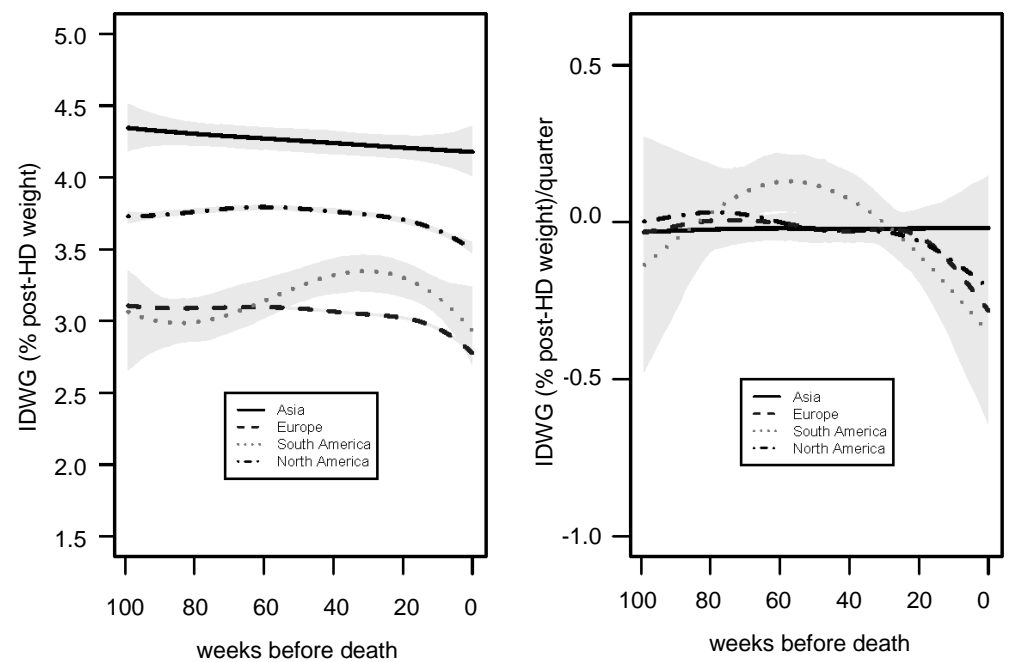

Figure 8.3b Mean IDWG (expressed as percent of post-HD weight) and 95\% confidence interval in male patients in the 104 weeks prior to death. 
In female patients from Europe, North and South America, SBP levels dropped between 7.8 (South America) and 12.4 (North America) $\mathrm{mmHg}$ in the 2 years preceding death [Figure 8.4a, left]; SBP decline was less pronounced in patients from Asia. The rate of SBP decline accelerated before death and was similar in patients from Europe, North and South America, but less pronounced in Asia [Figure 8.4a, right]. The results were materially identical in male patients, although the decline in Asian patients was not significant [Figure 8.4b].
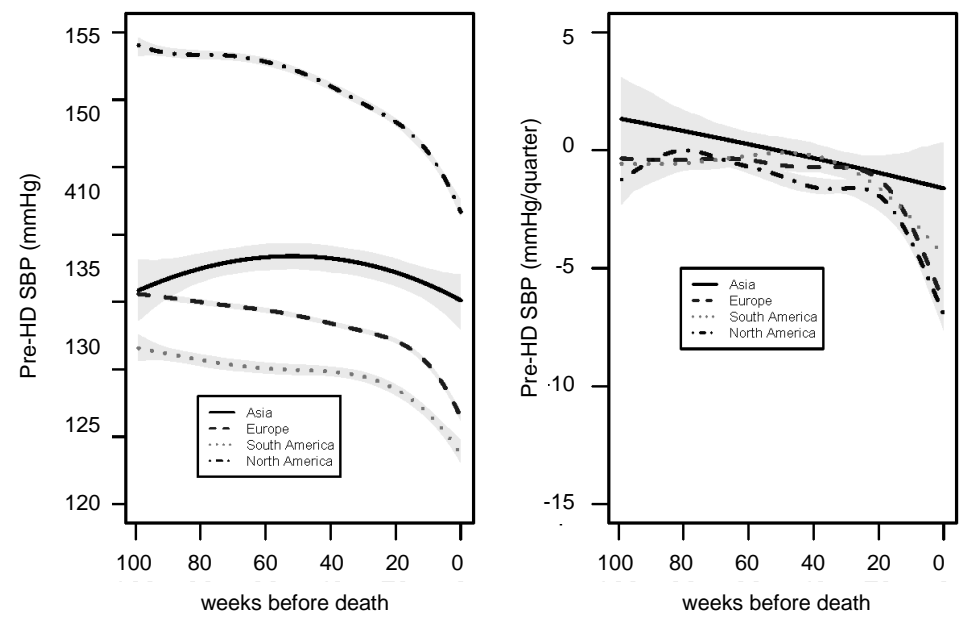

Figure 8.4a Mean pre-HD SBP and 95\% confidence intervals in female patients in the 104 weeks prior to death. The left panels show estimates of the partially conditional means with, the right panels show estimates of rate of change trajectories with $95 \%$ confidence intervals.
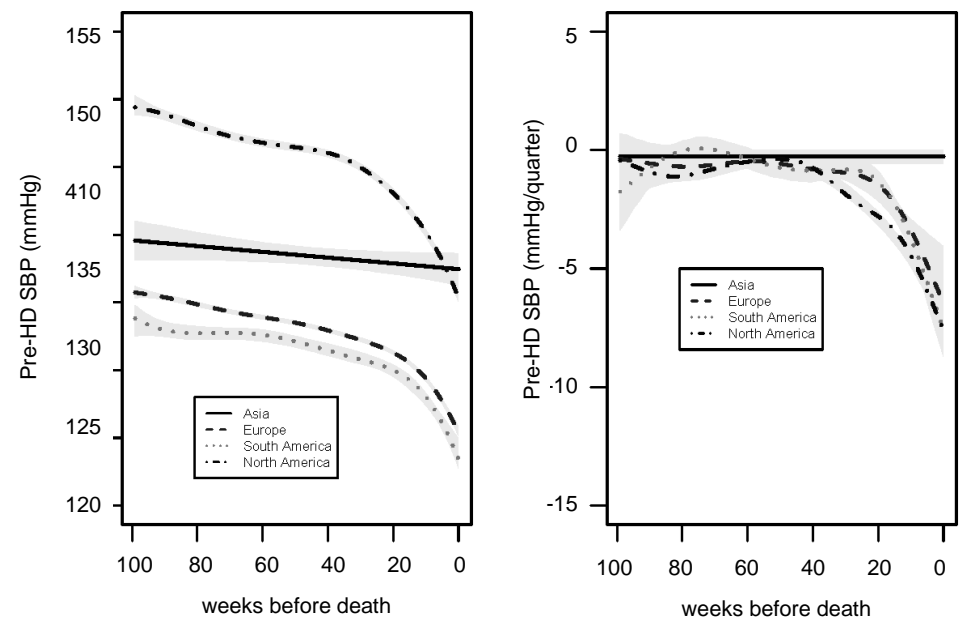

Figure 8.4b Mean pre-HD SBP and 95\% confidence intervals in male patients in the 104 weeks prior to death. 
CRP levels increased by $15 \mathrm{mg} / \mathrm{I}$ in the 2 years before death [Figure 8.5, left]. The rate of CRP increase accelerated before death and was similar in both genders [Figure 8.5, right].
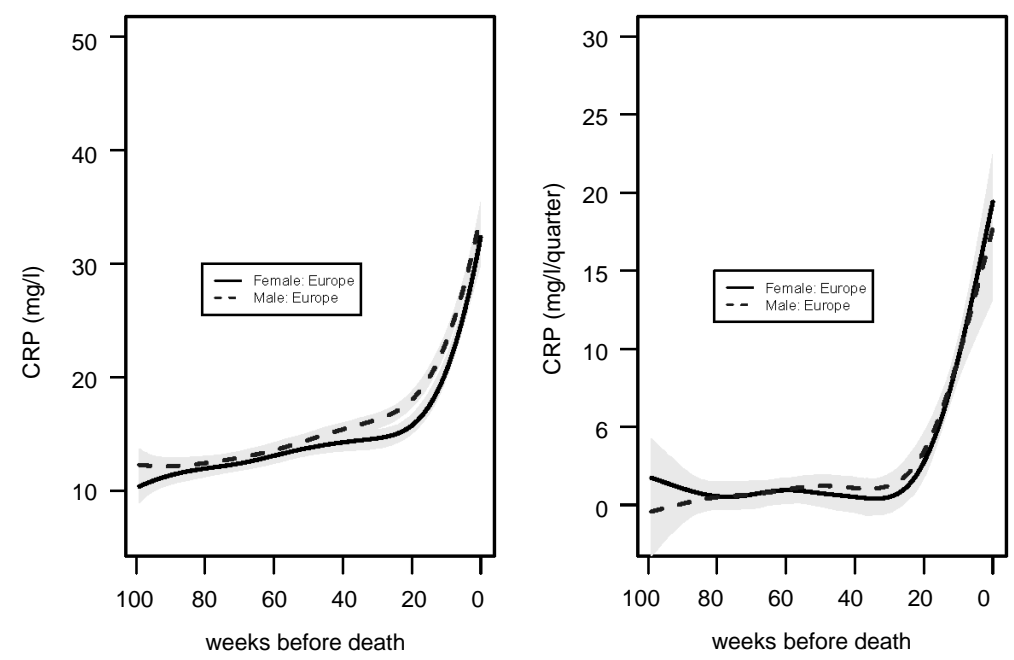

Figure 8.5 Mean CRP and 95\% confidence intervals in the 104 weeks prior to death. The left panel shows estimates of the partially conditional means with, the right panel shows estimates of rate of change trajectories with $95 \%$ confidence intervals.

Analysis of causes of deaths (cardio-vascular disease (CVD), infections, neoplasms and other) indicated that the majority of patients in each region died of CVD, with the highest proportion in North America (61\%) versus lowest in Asia (27\%). Neoplasms caused the lowest percentage of deaths in each region. Irrespective of the cause of death, dynamics of albumin, IDWG, SBP, and CRP were qualitatively comparable before death. [Supplemental figures A8.1 to A8.4]. In a separate analysis of patients who died of sudden death, we noted an increase in CRP and a decline in albumin before death in those patients (data not shown).

Because backward processes (i.e. slopes before death) and forward processes (i.e. slopes in surviving patients looking forward) cannot be compared directly, we conducted two analyses to elucidate parameter dynamics in survivors and nonsurvivors. In the first analysis, we selected incident patients who did not die during the study period and who survived $\geq 4$ years on dialysis. We graphed albumin, IDWG, SBP, and CRP in the first 2 years on dialysis in those patients to determine if any changes also exist in survivors [Figures 8.6 to 8.9 ]. In the surviving patients, albumin, IDWG, $\mathrm{SBP}$, and CRP did not appear to change in the first 2 years on HD. In fact, other than the initial minor increase in albumin, SBP, CRP, and IDWG remained stable throughout 
the observation period [Figures 8.6 to 8.9 , left]. The first derivatives of the trajectories also indicated no material change. [Figures 8.6 to 8.9 , right]. In the second analysis (Supplemental Information), we compared patients who survived to patients with the same vintage who died. Supplemental Figures A8.5a, A8.6a, A8.7a show incident patients who survived the first 2 years on dialysis (left panels) versus patients who survived the first 120 days but died between day 121 and end of 2 years on dialysis (right panels); Supplemental Figure A8.8a delineates CRP in survivors and nonsurvivors on the same chart. Supplemental Figures A8.5b, A8.6b, A8.7b show patients who survived the first 4 years on dialysis (left) versus patients who survived the first 2 years on dialysis but died between year 2 and 4 years (right); Supplemental Figure A8.8b delineates CRP in both survivors ( $>4$ years) and non-survivors. These results demonstrate notable differences in the patterns in the survivors versus patients who died during the same time period, with surviving patients generally showing no change, while non-survivors followed a declining (CRP rising) trajectory. In patients who died, the confidence intervals widen as the number of weeks from start on dialysis increases, because fewer patients are alive as time progresses.
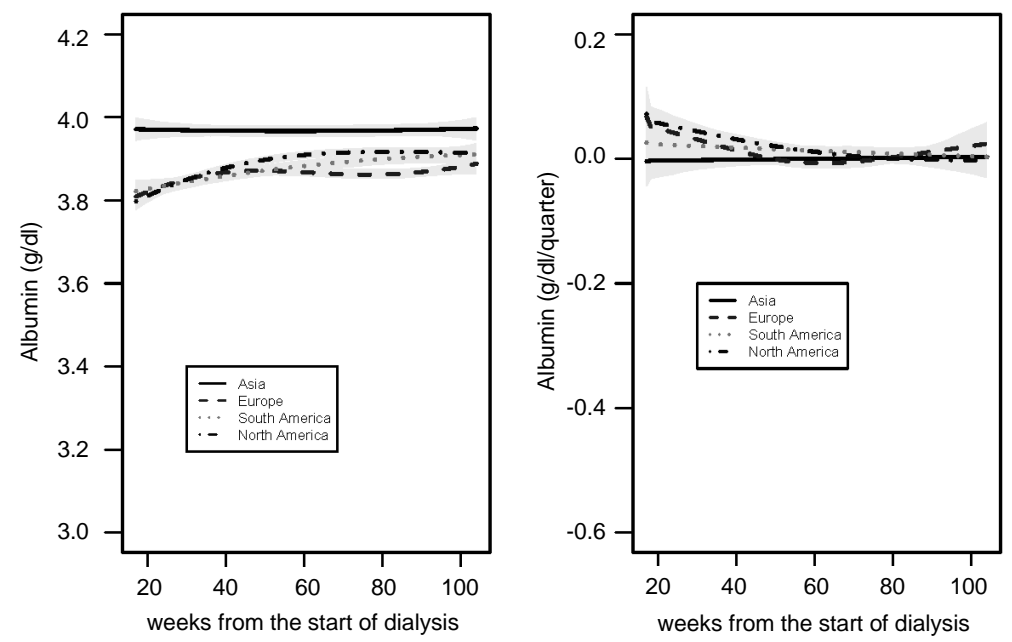

Figure 8.6a Mean serum albumin and 95\% confidence interval in the 104 weeks from the start of dialysis in female patients who survived $>4$ years. The left panels show estimates of the partially conditional means and right panels show estimates of rate of change trajectories with $95 \%$ confidence intervals. 

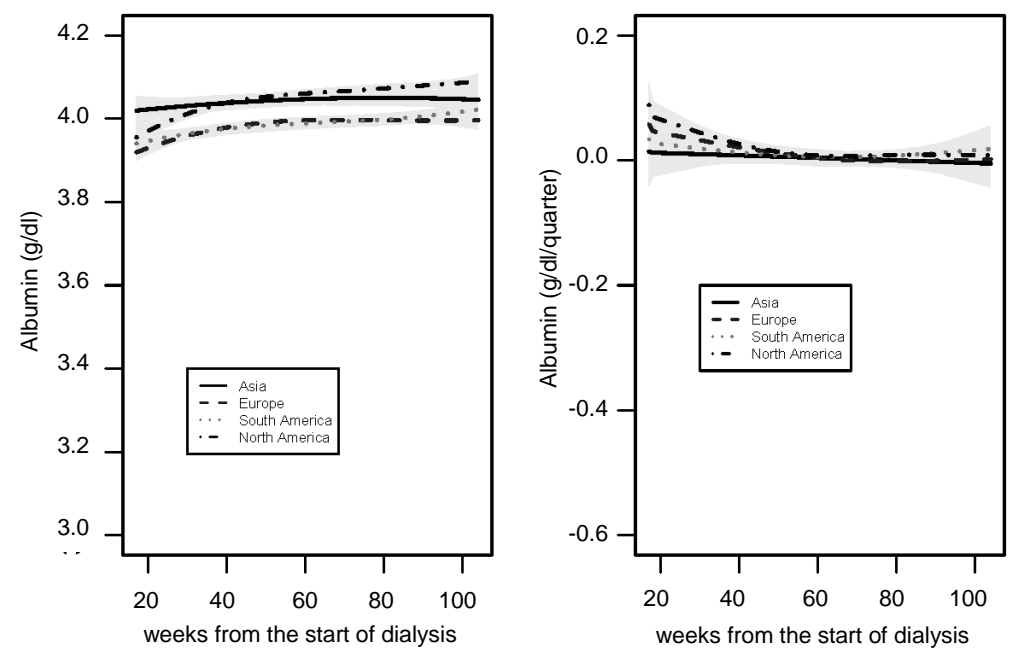

Figure 8.6b Mean serum albumin and 95\% confidence interval in the 104 weeks from the start of dialysis in male patients who survived $>4$ years.
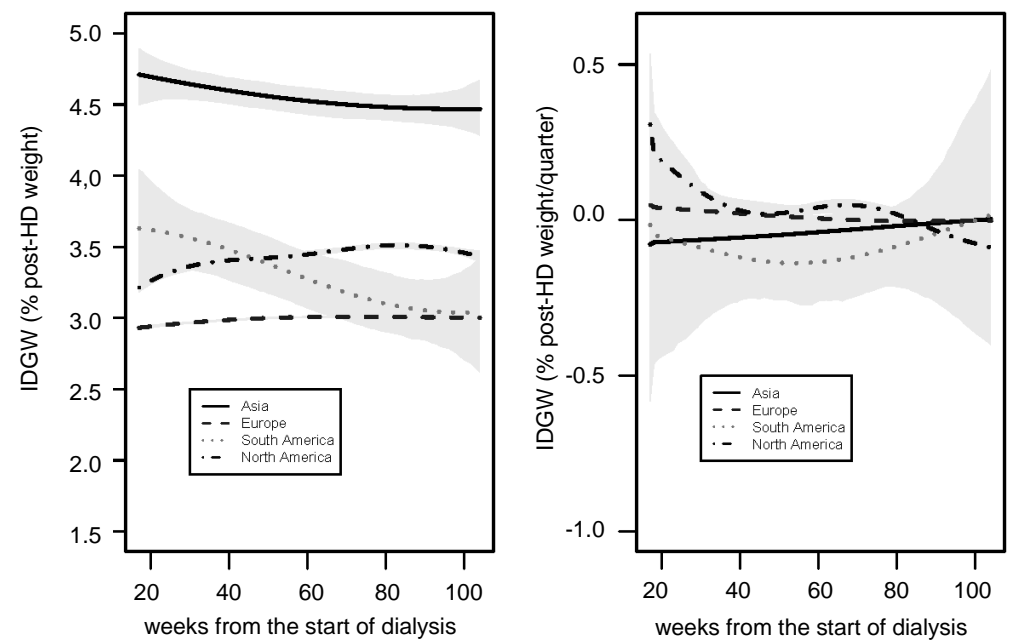

Figure 8.7a Mean IDWG (expressed as \% of post-HD weight) and 95\% confidence interval in the 104 weeks from the start of dialysis in female patients who survived $>4$ years. The left panels show estimates of the partially conditional means and right panels show estimates of rate of change. 

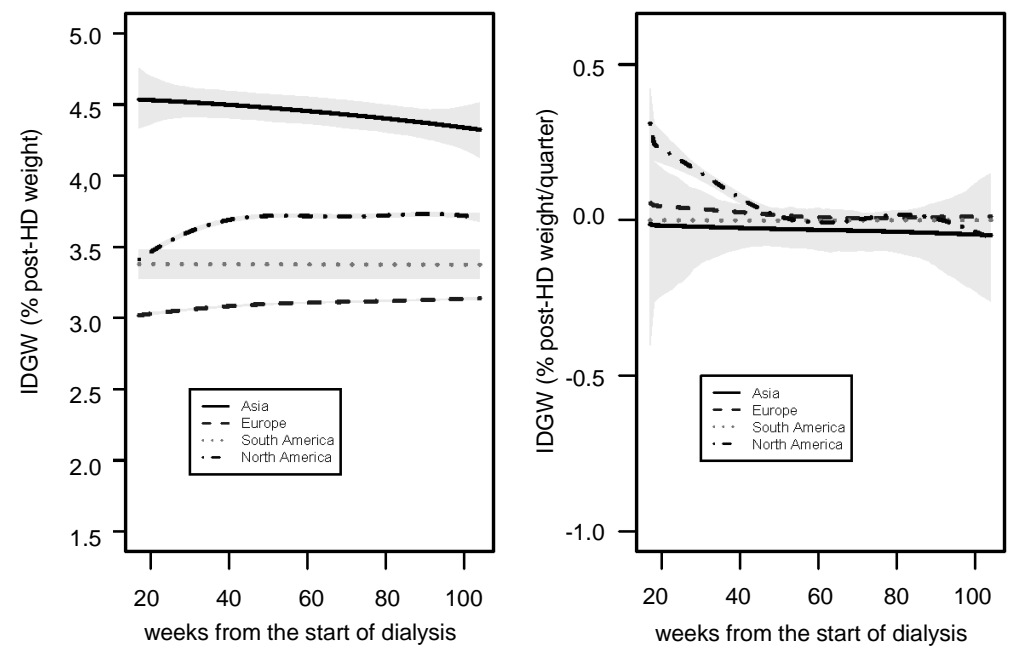

Figure 8.7b Mean IDWG (\%) and 95\% confidence interval in the 104 weeks from start of dialysis in male patients who survived $>4$ years.
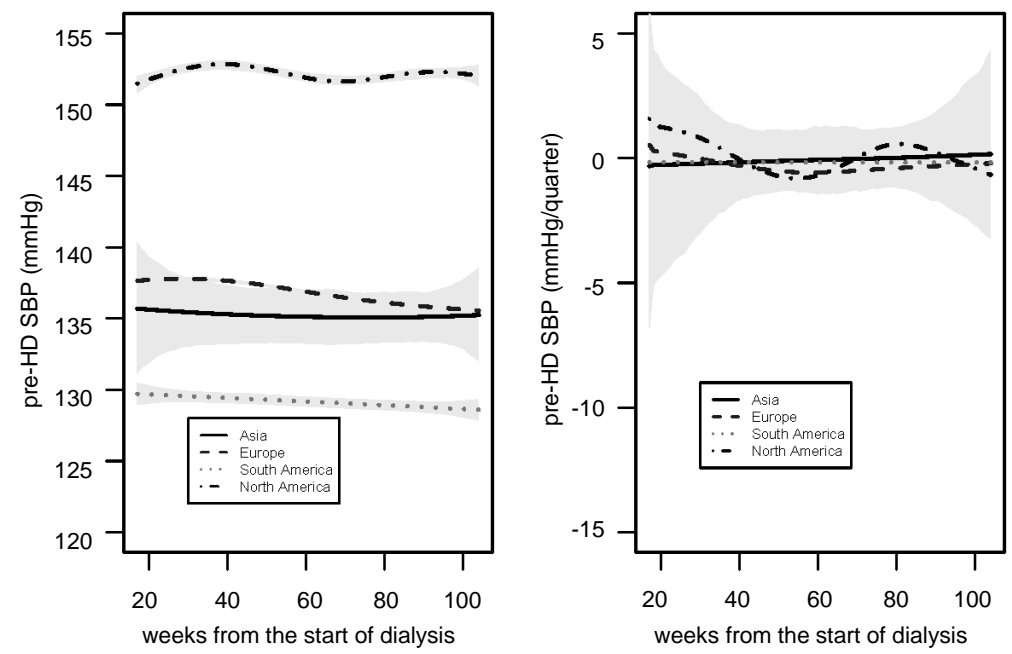

Figure 8.8a Mean pre-HD SBP and 95\% confidence interval in the 104 weeks from the start of dialysis in female patients who survived $>4$ years. The left panels show estimates of the partially conditional means and right panels show estimates of rate of change. 

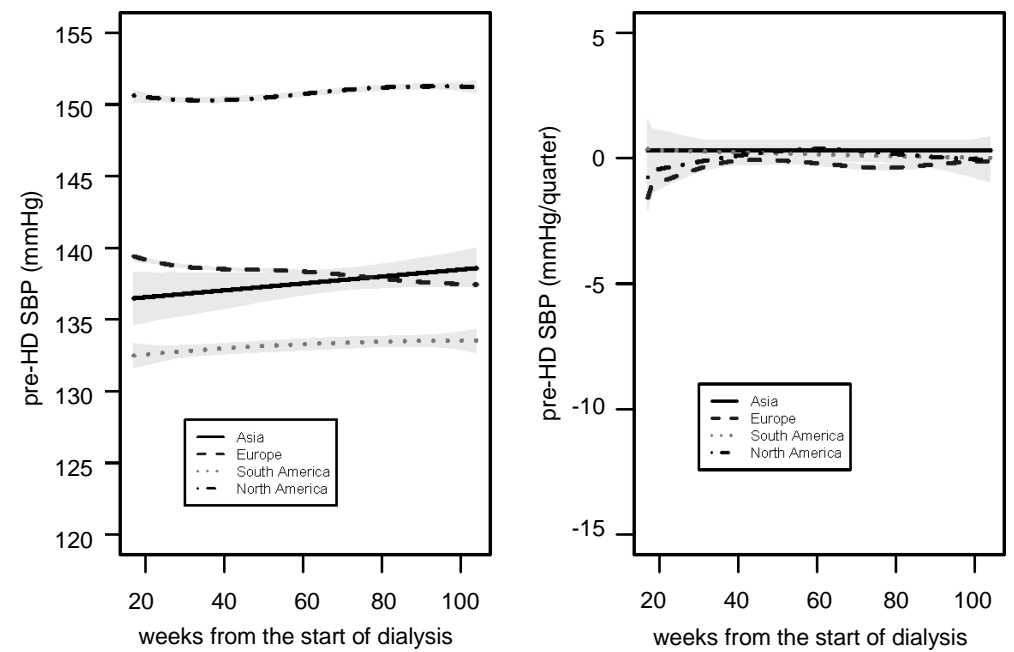

Figure 8.8b Mean pre-HD SBP and 95\% confidence interval in the 104 weeks from the start of dialysis in female patients who survived $>4$ years.
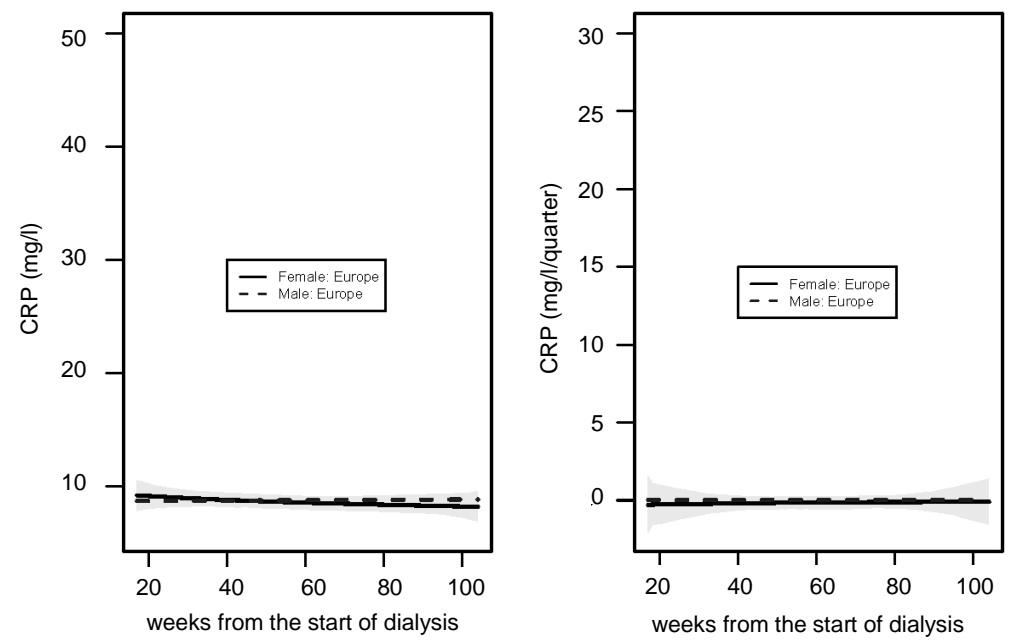

Figure 8.9 Mean CRP and 95\% confidence interval in the 104 weeks from the start of dialysis in patients who survived $>4$ years. The left panel shows estimates of the partially conditional means and right panel shows estimates of rate of change 


\section{Discussion}

In a study involving nearly 42,000 chronic HD patients from 25 countries and four continents we show evidence of comparable temporal evolutions of four important parameters prior to death. Regardless of geographical location, IDWG, SBP and serum albumin started to decline more than a year before death, with the decline in IDWG and SBP being less apparent in Asia. In the FMC Europe network, routine CRP measurements were available, which showed a steep increase before death.

To the best of our knowledge, this is the first study showing the dynamic changes of key clinical and laboratory parameters prior to death in highly diverse international cohorts of chronic HD patients. In a previous smaller study in 2,462 maintenance HD patients from Renal Research Institute (U.S.), comparable trends for post-HD weight and serum albumin were shown ${ }^{4}$. However, given the large variability of background mortality and treatment patterns throughout the world ${ }^{5-8}$, this study analyzed whether these trends were also apparent from a global perspective. Notably, most parameters assessed here started to deteriorate more than one year before death. Interestingly, a recent study indicated that functional status of HD patients remained stable until one month before death ${ }^{9}$. This temporal difference suggests that subtle changes in physiological and laboratory parameters may herald clinical deterioration in dialysis patients, possibly at a stage which is amenable to intervention.

The decline in serum albumin was apparent at an earlier stage in the present study as compared to the previous RRI study. However, this may be due to differences in statistical methodology between both studies. Whereas the previous study was based on linear mixed models, using linear spline functions with a knot point 12 weeks before death, the present study looked at all observations 104 weeks prior to death in a continuous fashion ${ }^{10}$. The stable albumin levels in incident surviving patients observed by us can be compared to results from the HEMO trial, where during the first 3 years of follow-up mean serum albumin levels declined by $0.21 \mathrm{~g} / \mathrm{dl}$. ${ }^{11}$ However, in contrast to our study, the HEMO trial enrolled prevalent patients with a vintage of $3.7 \pm 4.4$ years, rendering a direct comparison with our results difficult.

The pathophysiological mechanisms underlying these dynamics cannot be directly deduced from the present observational data. The four parameters were chosen because they may reflect changes in three key domains, namely cardiovascular function, nutrition, and inflammation, and because most of these indicators (except CRP) are readily available and are used in daily clinical practice.

The decline in SBP may reflect deterioration in cardiovascular function ${ }^{3}$, such as reduced cardiac output over time. The reduction in IDWG may reflect poorer appetite 
and diminished food intake ${ }^{12}$. In this respect, it may be more sensitive than post-HD weight, which usually represents the prescribed target weight. Importantly, body composition may change significantly in the absence of body weight changes. For instance, despite a decline in lean body mass, body weight may remain stable due to a concurrent increase in extracellular volume if post-HD target weight is not adjusted accordingly. The decline in IDWG and SBP before death is likely a reflection of "reverse epidemiology" ${ }^{13}$, and cannot not be used as an argument against the importance of blood pressure control and meticulous attention to sodium and fluid volume restriction in dialysis patients. Indeed, several studies have shown a relationship between high IDWG (as well as fluid overload) and hypertension and mortality ${ }^{14-16}$.

Serum albumin, a composite marker of nutrition and inflammation, and CRP are strong predictors of outcome ${ }^{17-19}$. The increase in CRP and the decline in the other three studied parameters occurred largely independently of age, gender, race, or region of the world (data on age and race not shown). Notable exceptions were the absence of declines in SBP in male, and in IDWG in both male and female Asian patients. The fact that SBP declined before death in all populations except in male HD patients from Asia is remarkable, and one may speculate that fluid overload is more prevalent in Asian patients close to death; unfortunately, this observation cannot be explained by the present data. It remains to be established whether environmental, treatment-related, genetic, and/or cardiac factors are involved in this phenomenon ${ }^{15}$, 20-22; the relatively small number of patients ( 787 males) may also be a factor. Different clinical practices related to target weight determination and monitoring may vary amongst the patients studied resulting in different blood pressure dynamics, particularly for malnourished patients whose target weight is not adjusted timely to their body weight.

Pre- and post-dialysis weights are only recorded once a month in the FMC Asia-Pacific network, so detailed information on IDWG may be missed. Studies in Southeast Asian populations living in the US may provide important additional insights. The substantially higher SBP seen in both surviving and non-surviving US patients compared to the other regions is impressive and deserves further investigation. We hypothesize that differences in fluid and anemia management and antihypertensive therapy may contribute to this observation ${ }^{23}$.

Analysis of trajectories of all factors measured before death by cause of death revealed no notable differences between patients dying from CVD, infections, or neoplasms. Neoplasms appear to be associated with the most striking dynamic trends in the four parameters studied. Declines in albumin and increases in CRP were also observed in patients who died of sudden death consistent with prior findings ${ }^{24}$. 
This international study may contribute to the understanding of the pathophysiological phenomena occurring before death in this highly vulnerable group of patients. The main message for the clinical practitioner is that the temporal evolutions of albumin, SBP, IDWG, and CPR are as important to follow as their absolute levels. This insight should also serve as a wake-up call for dialysis providers, since they may have to put information systems in place which allow the physicians not only to follow absolute levels of variables but also their dynamics. While a decline in albumin levels or rises in CRP usually alert healthcare providers, a consistent and unintended decline of SBP by more than 2-5 $\mathrm{mmHg}$ per quarter or IDWG drop by more than $0.2 \%$ per quarter may go unnoticed. On an operational level, the identification of temporal patterns can be supported by patient reports which depict both absolute levels and trends of variables. Moreover, the results of our study offer guidance for the development of predictive models. We speculate that it might be possible to construct multivariate algorithms by combining IDWG, SBP, and serum albumin levels that may be more predictive of the death risk than each individual parameter. Such models may appreciate even subtle intra-individual dynamics, such as mild drops of SBP over the course of months and a decline of serum albumin even within the normal range, which are likely to escape notice in a busy clinical setting. Paradoxically, some of these changes of key indicators may be welcomed as the result of successful interventions. Of course, lower IDWG may also be the outcome of successful fluid counseling and also of salt restriction, and SBP decline may indicate better blood pressure control. However, as our data show, these changes may also be ominous signs, and to differentiate these possibilities requires a keen clinical assessment of the patient.

Whether or not "alert systems" can trigger more timely and cost-effective interventions and improve clinical outcomes has to be addressed in future prospective trials. These alert systems can be based on various statistical methodologies and use a severity index to highlight patients at higher risk of death requiring an intervention. However, there is little doubt that understanding changes preceding death by many months is indispensable for the development of predictive models.

A potential limitation of the study is that a majority of the patients studied are treated in FMC clinics. On the other hand, in Europe patients from a different dialysis provider, KfH Germany, comprised a large component but provided similar results (not shown separately). Another limitation is the overrepresentation of patients from Europe and South America. We tried to address this issue by analyzing the data from each region separately. The current analysis is limited to four parameters only, which reflect cardiovascular, nutritional and inflammatory domains. Future studies will extend these analyses to other indicators, including measures of cardiac function and body composition. 
In conclusion, the temporal evolution of key clinical and laboratory indicators before death was found to be comparable in large international cohorts of chronic HD patients from four continents, with some exceptions in Asia. These findings raise the possibility that similar biological processes are operative in the months before death irrespective of gender, race, and region of the world. These findings may contribute to improved understanding of the factors leading to poor outcome in dialysis patients and aid the development of risk prediction models which may facilitate timely interventions and hopefully improve outcomes.

\section{Methods}

\section{Participants and study design}

In this retrospective cohort study we identified all chronic HD patients from 25 countries in five continents treated in clinics of Fresenius Medical Care (FMC) from Europe (17 countries), FMC Latin America (Argentina only), FMC Asia-Pacific (South East Asia and Australia, 5 countries), Renal Research Institute (U.S.), and the Kuratorium für Dialyse und Nierentransplantation e.V. ( $\mathrm{KfH}, \mathrm{Germany}$ ) between Jan 1, 2000 and Dec 31, 2010 ("study period"). The data from these provider databases were aggregated into continents: FMC Europe and KfH Germany is titled "Europe"; FMC Latin America data is titled "South America;" FMC Asia-Pacific data is titled "Asia;" and data from RRI is represented as "North America."

Patients with at least one HD in-center treatment during the study period were stratified into incident (patients with first treatment recorded within 90 days of first ever dialysis) and prevalent. We conducted two separate analyses: the first looked at incident and prevalent patients who died during the study period $(\mathrm{N}=41,903)$, and the second looked at incident patients who did not die during the study period and survived more than four years on dialysis ["censored" patients] $(\mathrm{N}=10,277)$. These two cohorts were chosen to assure that the two analyzed patient cohorts (survivors and non-survivors) did not overlap. Figure 8.1 presents the flowchart of the study design. Additional results are presented in Supplemental Information, where we separate the dynamics before death by cause of death and compare the patients who survived to those who died with the same vintage. For the figures, only the continents with more than 250 patients were included (thus excluding Australia). Furthermore, all figures include only continents with more than 50 weeks of available observations with more than 10 patients in each week. 


\section{Data flow and analysis}

In most databases, clinical data were transferred manually from the respective data sheets into a master database. Multiple levels of internal quality control measures were in place to guarantee data consistency and validity. In most European clinics and all of the RRI clinics, laboratory data were directly imported electronically from the clinical laboratories. $\mathrm{KfH}$ (Germany) data were analyzed locally by statisticians working with this particular network; all other data were analyzed centrally at the Department of Statistics and Applied Probability, University of California Santa Barbara, CA, U.S.. The same validated program codes were used by all statisticians.

\section{Clinical parameters}

Pre- and post HD body weight was measured using scales calibrated according to the respective national standards. IDWG in percent of post-HD body weight was calculated as the difference between pre-HD weight and the preceding post-HD weight divided by the preceding post-HD weight. In the European and North American data, SBP and IDWG are available for each dialysis session; in Asia and South America these data were available monthly. Of note, for Asia and South America, this meant that post-dialysis weight from the previous month was used in calculation of IDWG. SBP was assessed by automated devices in all patients in U.S., Asia, and Europe. Manual measurement of SBP was the standard procedure in South America. In all databases, SBP was measured with patients at rest in a supine or sitting position.

\section{Laboratory analysis}

In most European clinics and all of the RRI clinics, laboratory data were directly imported electronically from the clinical laboratories. The MONDO data base compiles information on laboratory assays used. Serum albumin was measured by nephelometry in $\mathrm{KfH}$ (Germany) clinics and by the bromocresol green (BCG) method in all European clinics (except for Portugal), most Asian clinics, South America and the U.S.. Both methods are calibrated to the international standard ERM DA 470k/IFCC. The bromocresol purple (BCP) method was used in Portugal and some HD clinics in Asia. To ensure comparability between the two methods, BCP measurements were transformed to BCG values using a published formula (concentrations in $\mathrm{g} / \mathrm{dl})^{25}$ : Albumin $_{\mathrm{BCG}}=$ Albumin $_{\mathrm{BCP}}+0.55$. Serum albumin levels were measured at regular intervals, ranging from monthly to quarterly. CRP was measured routinely only in FMC Europe clinics. 


\section{Statistical analysis}

For descriptive purposes, means and standard deviations of continuous variables and percentages for categorical variables are presented. For the analysis of the variables of interest (IDWG, SBP, CRP and albumin), we considered the backward processes that start at death and go backward in time ${ }^{10} . \mathrm{Y}(\mathrm{u})$ is denoted as the backward process for a patient $\mathrm{u}$ weeks (or months) before death, and $\mathrm{T}$ as the survival time of this patient from the initiation of dialysis. We were interested in estimating the partially conditional mean $E(Y(u) \mid T>u)$ for a period of two years before death ${ }^{26}$. The partially conditional mean $E(Y(u) \mid T>u$ ) represents the expectation of the backward process $u$ weeks before death conditional on the patient having survived longer than $u$ weeks. For weekly measurements, the two-year period was divided into 104 weeks. For a fixed $1 \leq u \leq 104$ weeks, we estimated $E(Y(u) \mid T>u)$ by the average measurements among patients who survived longer than u weeks. The weekly (or monthly) estimates of partially conditional means were rough. To further investigate the trend of the partially conditional means and their trajectories before death, we fitted the estimated weekly partially conditional means using quintic splines and estimated the trajectories of the first derivatives ("velocity"), which represent the rate of change of the variable. The $\mathrm{R}$ package ASSIST was used to fit the quintic spline where the smoothing parameter was selected by the generalized maximum likelihood method. Bootstrap confidence intervals were constructed as described $\mathrm{in}^{27}$.

Similar spline functions were fitted in the forward analysis looking at all incident patients who survived more than four years: data for IDWG, albumin, CRP and SBP were analyzed over a period of 2 years (104 weeks) from the start of dialysis. This cohort was chosen to avoid overlap with the backward process. Similar methodology was applied to figures shown in Supplemental Information. All computations were made with $\mathrm{R}$ statistics software system version $2.11 .1^{28}$. 


\section{Supplemental Information}

Supplemental information contains additional figures that separate dynamics of albumin, pre-dialysis systolic blood pressure (SBP), interdialytic weight gain (IDWG), and C-reactive protein (CRP) by causes of death (Supplemental Figures A8.1 to A8.4). We also present the dynamics of patients who survived versus those who died over the same vintage (Supplemental Figures A8.5 to A8.8).
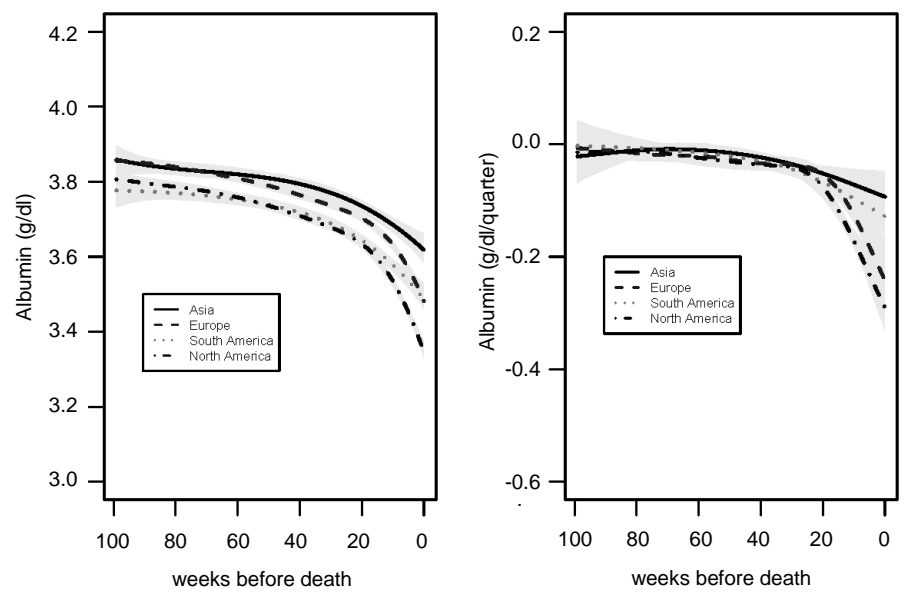

Figure A8.1a Mean serum albumin and 95\% confidence interval in the 104 weeks prior to death for CVD deaths. The left panels show estimates of the partially conditional means with $95 \%$ confidence intervals, the right panels show estimates of rate of change trajectories with $95 \%$ confidence intervals.
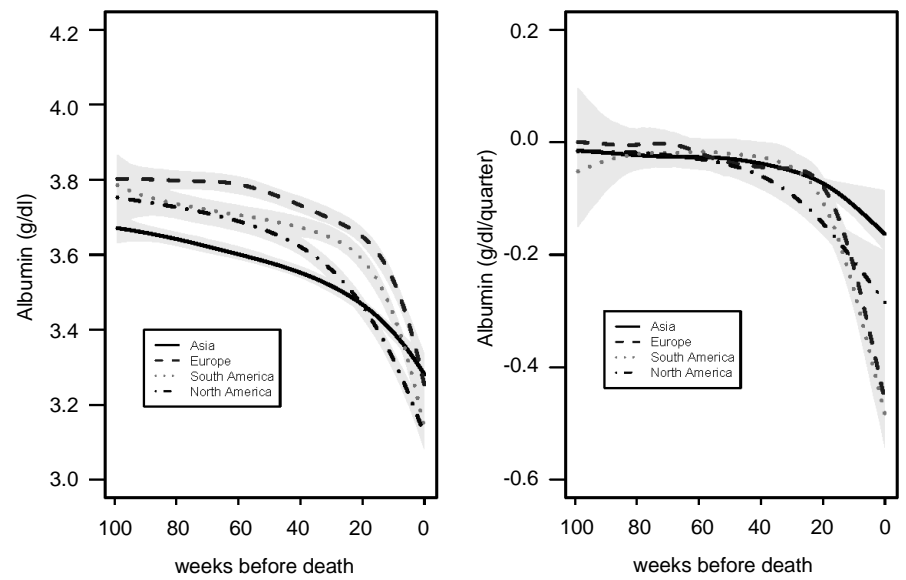

Figure A8.1b Mean serum albumin and 95\% confidence interval in the 104 weeks prior to death for infectious deaths. 

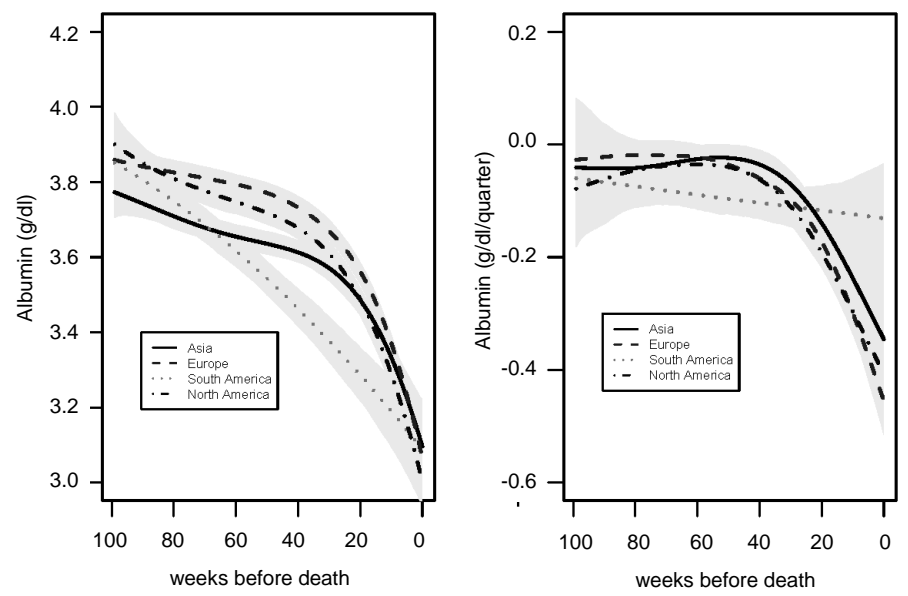

Figure A8.1c Mean serum albumin and 95\% confidence interval in the 104 weeks prior to death for neoplasm-related deaths.
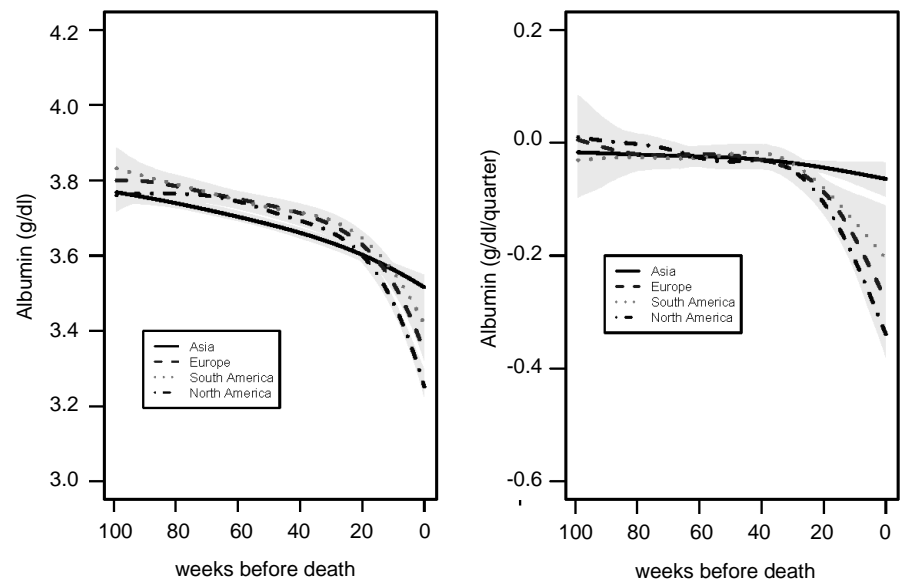

Figure A8.1d Mean serum albumin and 95\% confidence interval in the 104 weeks prior to death for other causes of death. 

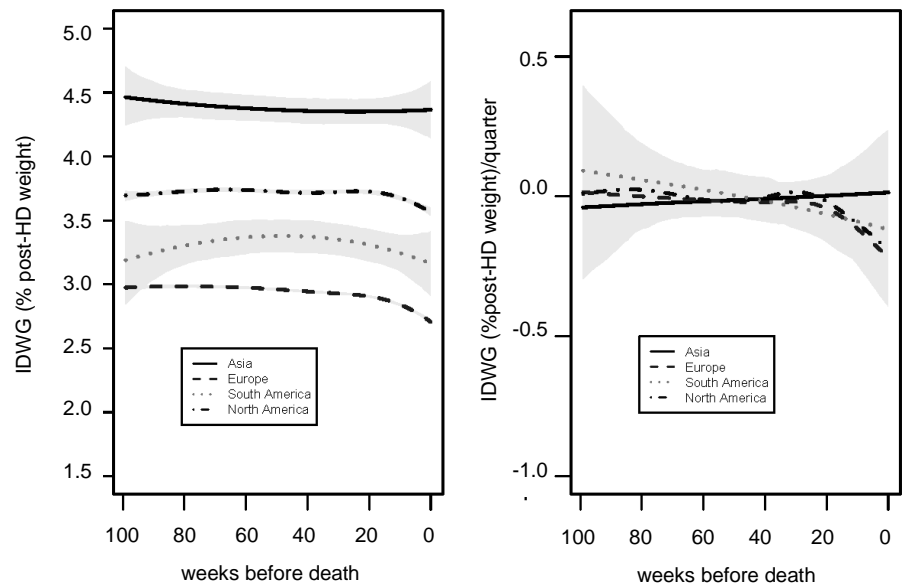

Figure A8.2a Mean IDWG (expressed as \% of post-HD weight) and 95\% confidence interval in the 104 weeks prior to death for CVD deaths. The left panels show estimates of the partially conditional means with $95 \%$ confidence intervals, the right panels show estimates of rate of change trajectories with $95 \%$ confidence intervals.
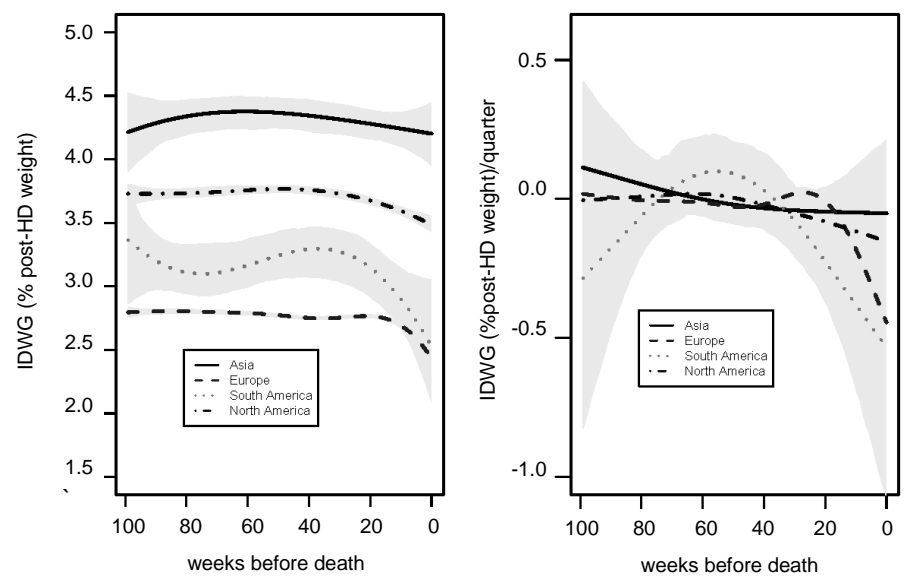

Figure A8.2b: Mean IDWG (expressed as \% of post-HD weight) and 95\% confidence interval in the 104 weeks prior to death for infectious deaths. 

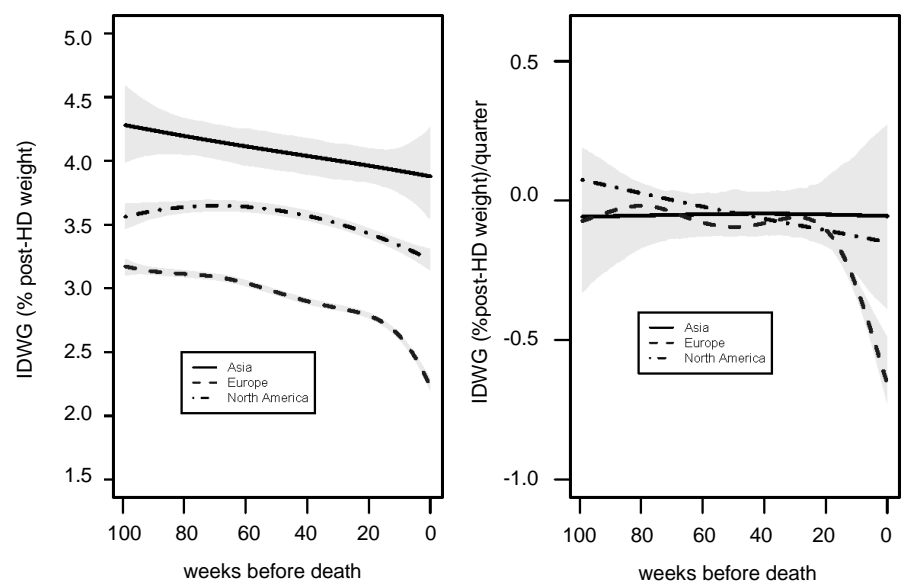

Figure A8.2c Mean IDWG (expressed as \% of post-HD weight) and 95\% confidence interval in the 104 weeks prior to death for neoplasm-related deaths.
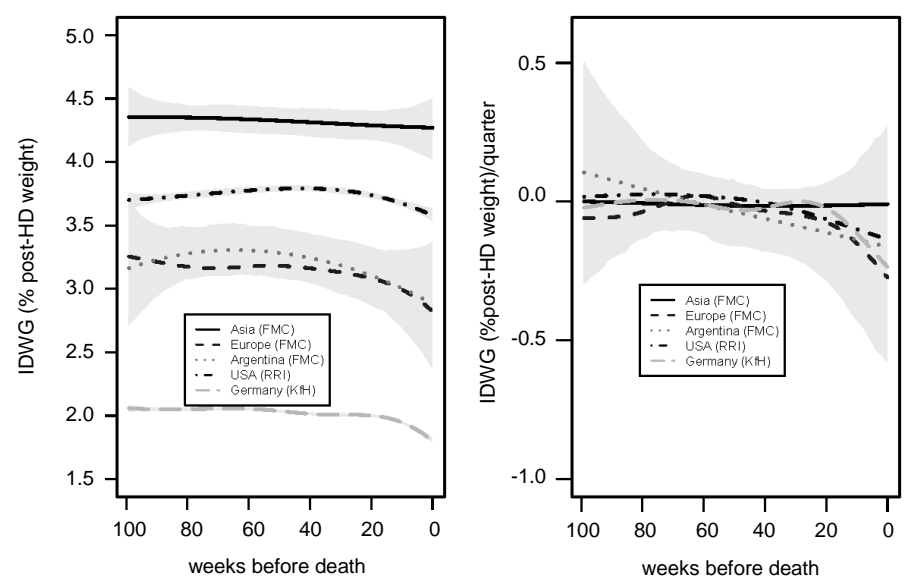

Figure A8.2d Mean IDWG (expressed as \% of post-HD weight) and 95\% confidence interval in the 104 weeks prior to death for other causes of death. 

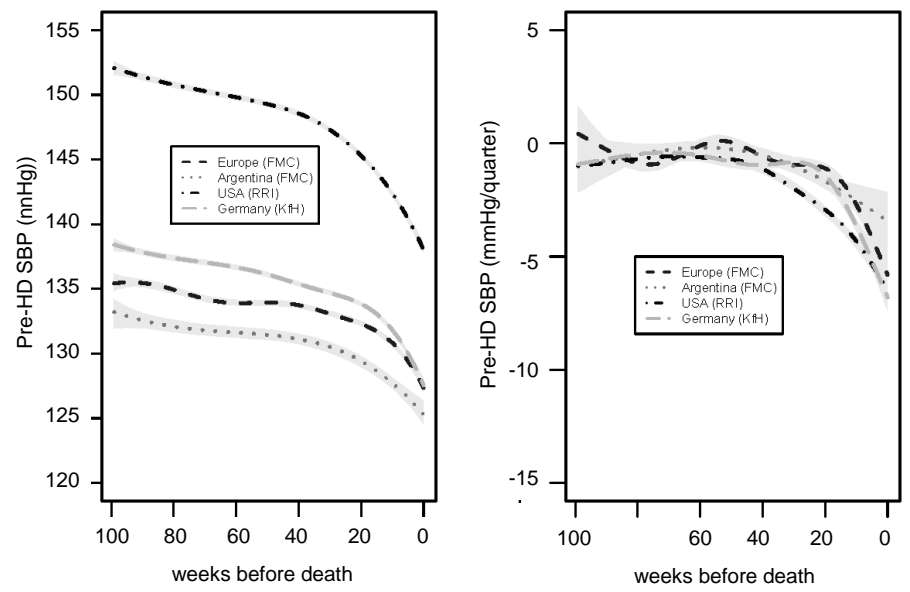

Figure A8.3a Mean pre-HD SBP and 95\% confidence interval in the 104 weeks prior to death for CVD deaths. The left panels show estimates of the partially conditional means with $95 \%$ confidence intervals, the right panels show estimates of rate of change trajectories with $95 \%$ confidence intervals.
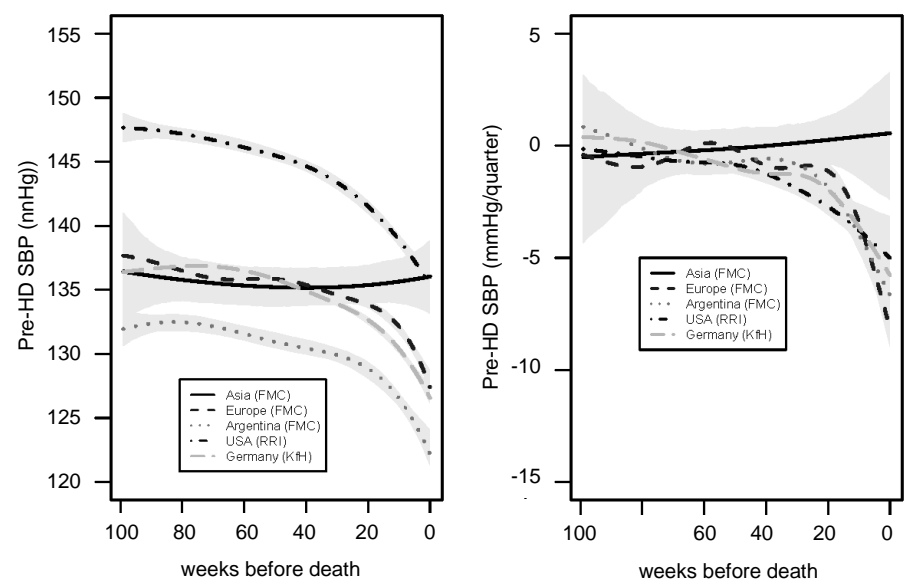

Figure A8.3b Mean pre-HD SBP and 95\% confidence interval in the 104 weeks prior to death for infectious deaths. 

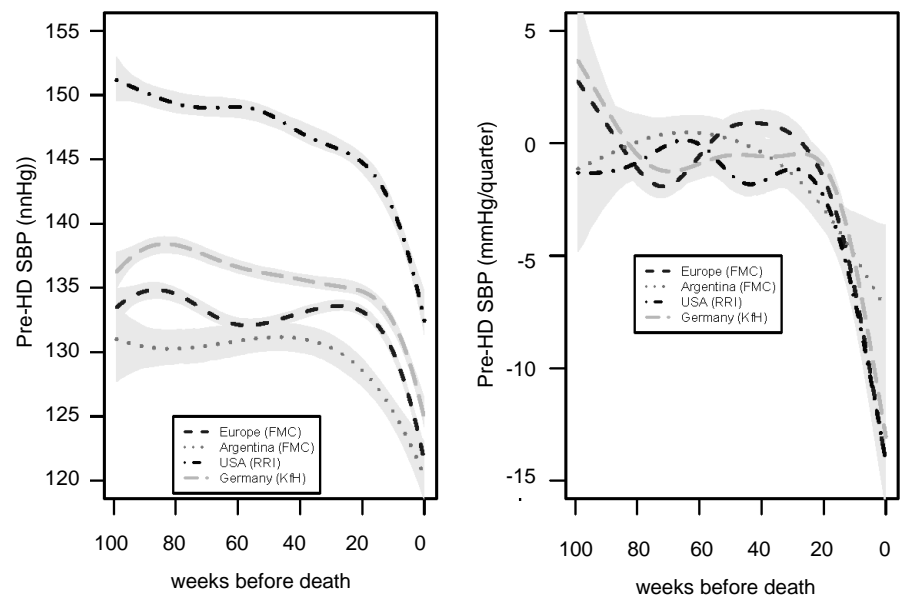

Figure A8.3c Mean pre-HD SBP and 95\% confidence interval in the 104 weeks prior to death for neoplasmrelated deaths.
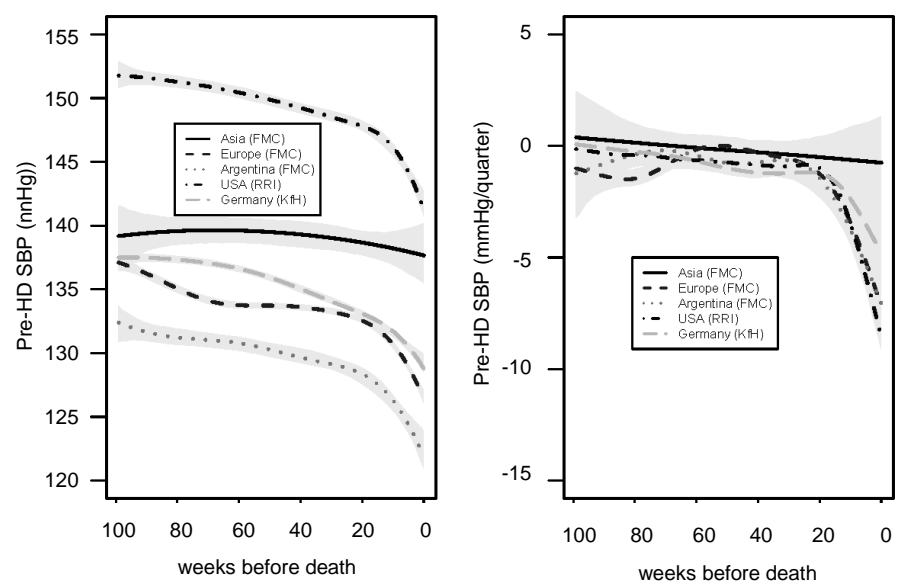

Figure A8.3d Mean pre-HD SBP and 95\% confidence interval in the 104 weeks prior to death for other causes of death. 

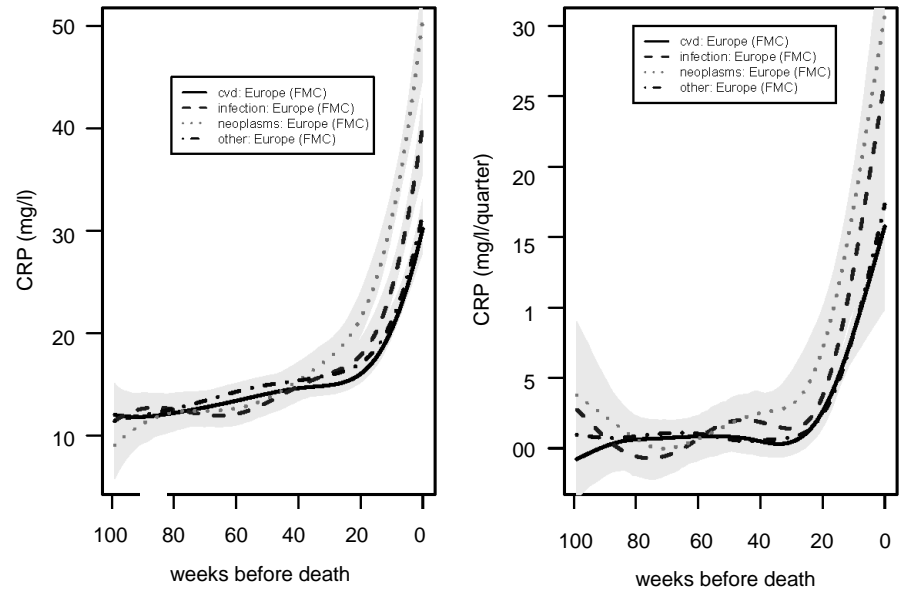

Figure A8.4 Mean CRP and 95\% confidence interval in the 104 weeks prior to death separated by cause of death. The left panels show estimates of the partially conditional means with $95 \%$ confidence intervals, the right panels show estimates of rate of change trajectories with $95 \%$ confidence intervals.
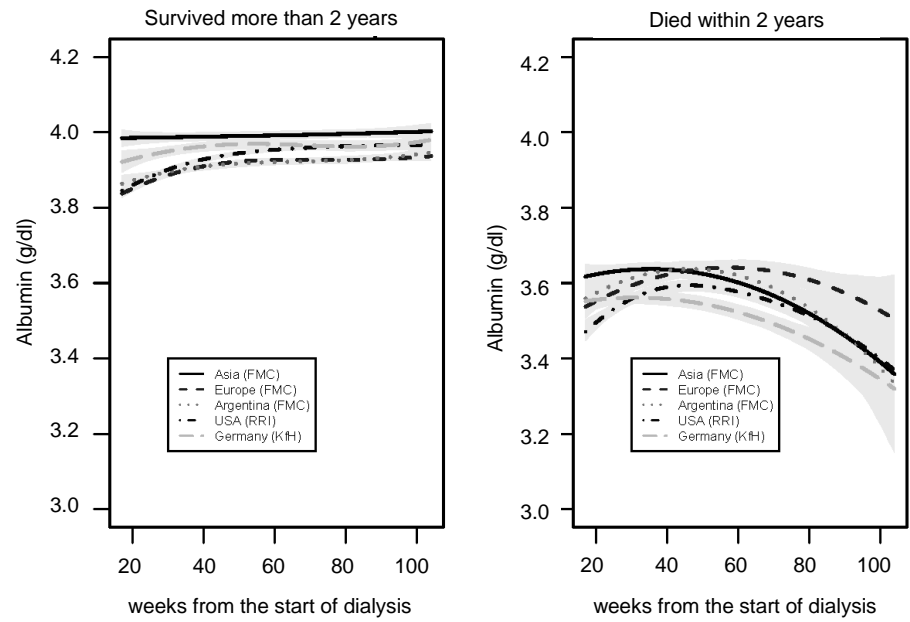

Figure A8.5a Mean serum albumin and 95\% confidence interval in the 104 weeks from the start of dialysis in patients who survived $>2$ years (left) versus patients who survived first 120 days on dialysis but died in first 104 weeks (right). 

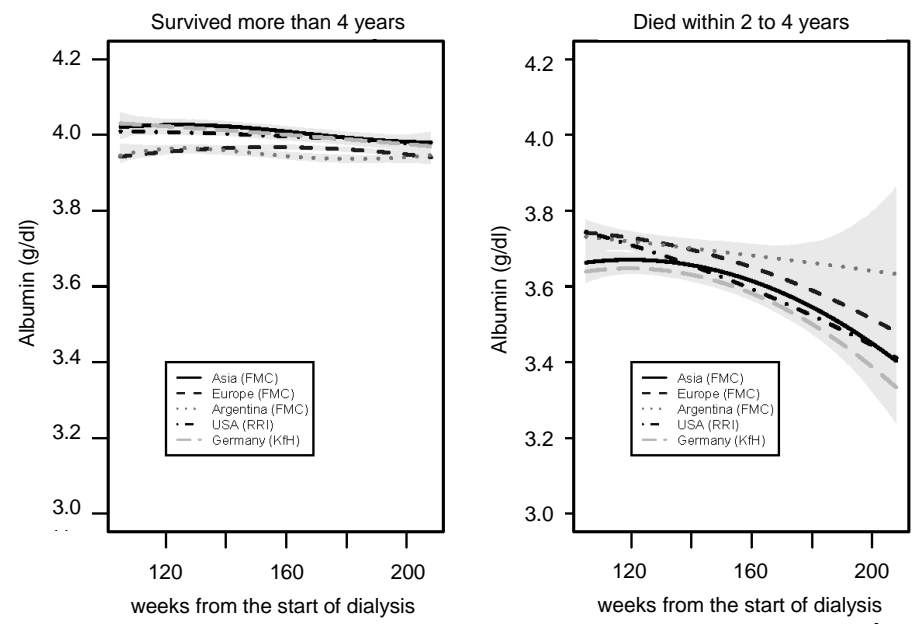

Figure A8.5b Mean serum albumin and 95\% confidence interval in weeks 105 to 208 weeks from the start of dialysis in patients who survived $>4$ years (left) versus patients who survived first 104 weeks on dialysis but died in weeks 105 to 208 (right).
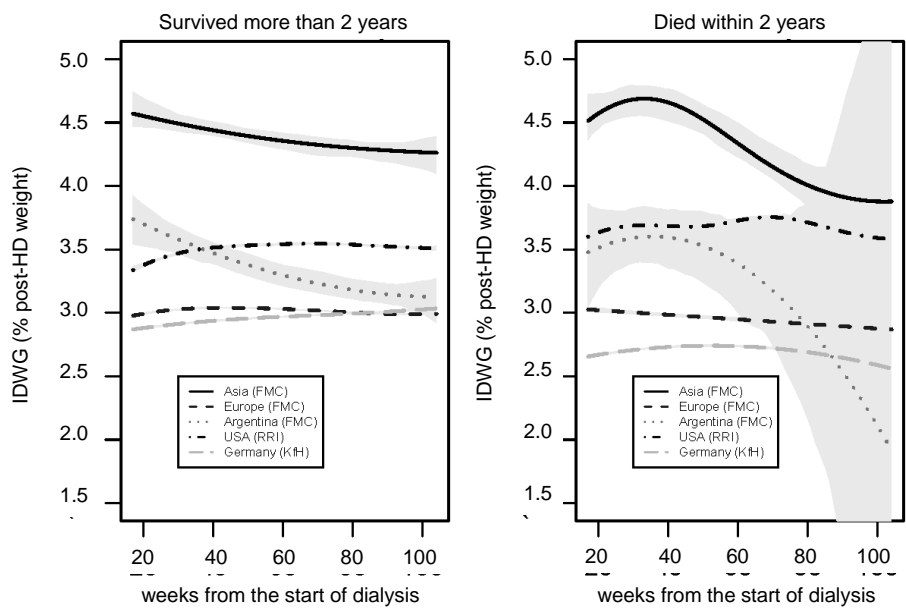

Figure A8.6a Mean IDWG (expressed as \% of post-HD weight) and 95\% confidence interval in the 104 weeks from the start of dialysis in patients who survived $>2$ years (left) versus patients who survived first 120 days on dialysis but died in first 104 weeks (right). 

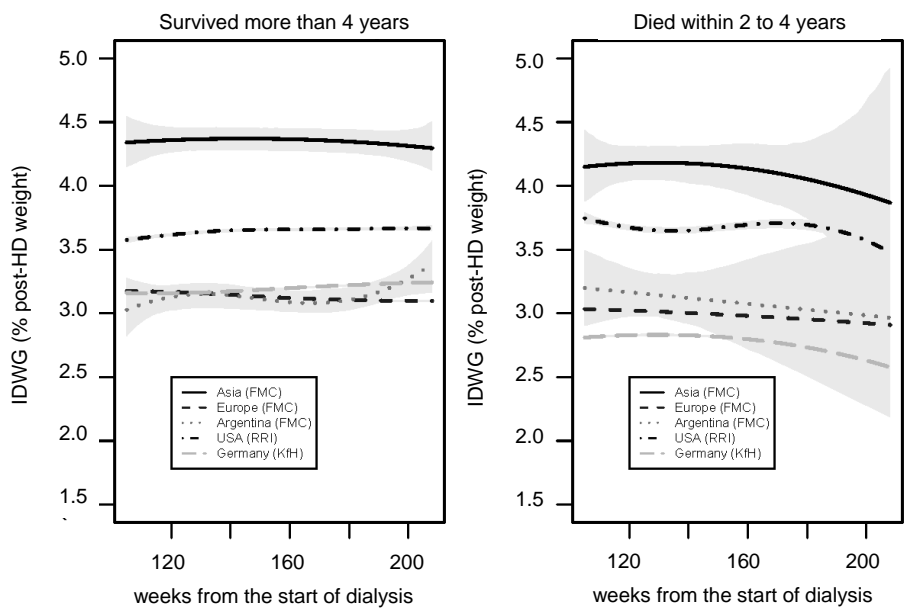

Figure A8.6b Mean IDWG (expressed as \% of post-HD weight) and 95\% confidence interval in weeks 105 to 208 weeks from the start of dialysis in patients who survived $>4$ years (left) versus patients who survived first 104 weeks on dialysis but died in weeks 105 to 208 (right).
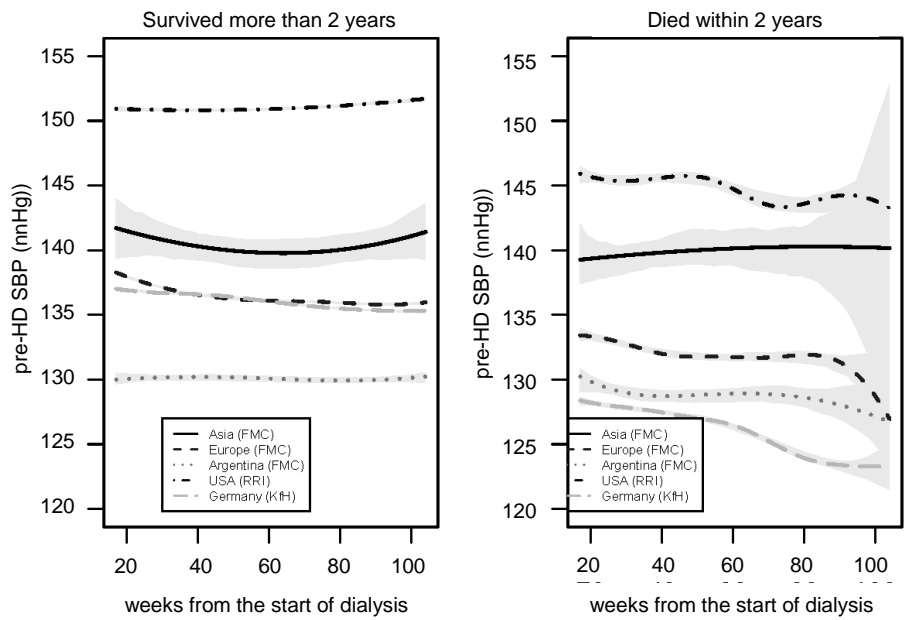

Figure A8.7a Mean pre-HD SBP and 95\% confidence interval in the 104 weeks from the start of dialysis in patients who survived $>2$ years (left) versus patients who survived first 120 days on dialysis but died in first 104 weeks (right). 

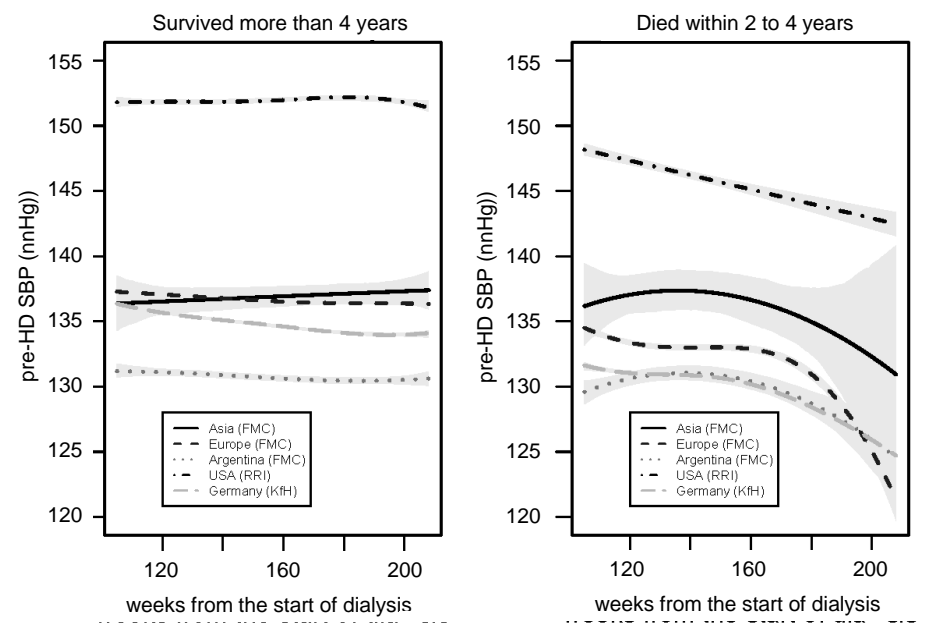

Figure A8.7b Mean pre-HD SBP and 95\% confidence interval in weeks 105 to 208 weeks from the start of dialysis in patients who survived $>4$ years (left) versus patients who survived first 104 weeks on dialysis but died in weeks 105 to 208 (right).

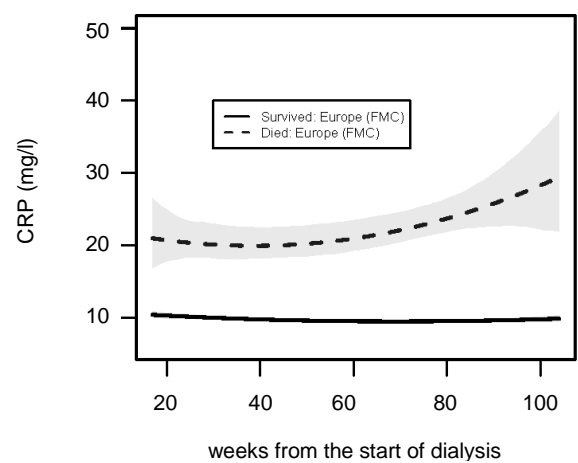

Figure A8.8a Mean CRP and 95\% confidence interval in the 104 weeks from the start of dialysis in patients who survived $>2$ years and patients who survived first 120 days on dialysis but died in first 104 weeks. 
Chapter 8

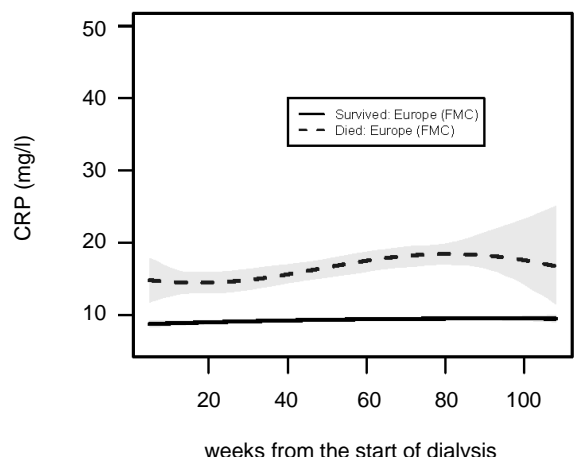

Figure A8.8b Mean CRP and 95\% confidence interval in weeks 105 to 208 weeks from the start of dialysis in patients who survived $>4$ years and patients who survived first 104 weeks on dialysis but died in weeks 105 to 208 . 


\section{References}

1. Himmelfarb J, Kliger AS. End-stage renal disease measures of quality. Annu Rev Med. 2007;58:387-99.

2. Raimann JG, Usvyat LA, Thijssen S, et al. Blood pressure stability in hemodialysis patients confers a survival advantage: results from a large retrospective cohort study. Kidney Int. 2011;81:548-58.

3. Li Z, Lacson E, Jr., Lowrie EG, et al. The epidemiology of systolic blood pressure and death risk in hemodialysis patients. Am J Kidney Dis. 2006;48:606-15.

4. Kotanko P, Thijssen S, Usvyat L, et al. Temporal evolution of clinical parameters before death in dialysis patients: a new concept. Blood Purif. 2009;27:38-47.

5. Yoshino $M$, Kuhlmann MK, Kotanko $P$, et al. International differences in dialysis mortality reflect background general population atherosclerotic cardiovascular mortality. J Am Soc Nephrol. 2006;17:3510-9.

6. Pisoni RL, Greenwood RN. Selected lessons learned from the Dialysis Outcomes and Practice Patterns Study (DOPPS). Contrib Nephrol. 2005;149:58-68.

7. Pifer TB, McCullough KP, Port FK, et al. Mortality risk in hemodialysis patients and changes in nutritional indicators: DOPPS. Kidney Int. 2002;62:2238-45.

8. Goodkin DA, Bragg-Gresham JL, Koenig KG, et al. Association of comorbid conditions and mortality in hemodialysis patients in Europe, Japan, and the United States: the Dialysis Outcomes and Practice Patterns Study (DOPPS). J Am Soc Nephrol. 2003;14:3270-7.

9. Murtagh FE, Addington-Hall JM, Higginson IJ. End-stage renal disease: a new trajectory of functional decline in the last year of life. J Am Geriatr Soc. 2011;59:304-8.

10. Gary Chan KC, Wang MC. Backward Estimation of Stochastic Processes with Failure Events as Time Origins. Ann Appl Stat. 2010;4:1602-20.

11. Rocco MV, Dwyer JT, Larive B, et al. The effect of dialysis dose and membrane flux on nutritional parameters in hemodialysis patients: results of the HEMO Study. Kidney Int. 2004;65:2321-34.

12. Lopez-Gomez JM, Villaverde M, Jofre R, Rodriguez-Benitez P, Perez-Garcia R. Interdialytic weight gain as a marker of blood pressure, nutrition, and survival in hemodialysis patients. Kidney Int Suppl. 2005(93):S63-8.

13. Kalantar-Zadeh K. What is so bad about reverse epidemiology anyway? Semin Dial. 2007;20:593-601.

14. Kalantar-Zadeh K, Regidor DL, Kovesdy CP, et al. Fluid retention is associated with cardiovascular mortality in patients undergoing long-term hemodialysis. Circulation. 2009;119:671-9.

15. Ozdogan O, Kayikcioglu M, Asci G, et al. Left atrial volume predicts mortality in low-risk dialysis population on long-term low-salt diet. Am Heart J. 2010;159:1089-94.

16. Wizemann V, Wabel $\mathrm{P}$, Chamney $\mathrm{P}$, et al. The mortality risk of overhydration in haemodialysis patients. Nephrol Dial Transplant. 2009;24:1574-9.

17. Kaysen GA, Dubin JA, Muller HG, Mitch WE, Rosales LM, Levin NW. Relationships among inflammation nutrition and physiologic mechanisms establishing albumin levels in hemodialysis patients. Kidney Int. 2002;61:2240-9.

18. Yeun JY, Levine RA, Mantadilok V, Kaysen GA. C-Reactive protein predicts all-cause and cardiovascular mortality in hemodialysis patients. Am J Kidney Dis. 2000;35:469-76.

19. Owen WF, Lowrie EG. C-reactive protein as an outcome predictor for maintenance hemodialysis patients. Kidney Int. 1998;54:627-36.

20. Lyratzopoulos G, McElduff P, Heller RF, Hanily M, Lewis PS. Comparative levels and time trends in blood pressure, total cholesterol, body mass index and smoking among Caucasian and South-Asian participants of a UK primary-care based cardiovascular risk factor screening programme. BMC Public Health. 2005;5:125.

21. Kelly TN, Hixson JE, Rao DC, et al. Genome-wide linkage and positional candidate gene study of blood pressure response to dietary potassium intervention: the genetic epidemiology network of salt sensitivity study. Circ Cardiovasc Genet. 2010;3:539-47.

22. Yap IK, Brown IJ, Chan $Q$, et al. Metabolome-wide association study identifies multiple biomarkers that discriminate north and south Chinese populations at differing risks of cardiovascular disease: INTERMAP study. J Proteome Res. 2010;9:6647-54. 
23. Guinsburg A ea. Comparison of blood pressure patterns in incident hemodialysis patients: evaluation of dialysis data from 24 countries. Paper presented at: ERA-EDTA, 2012; Paris.

24. Parekh RS, Plantinga LC, Kao WH, et al. The association of sudden cardiac death with inflammation and other traditional risk factors. Kidney Int. 2008;74:1335-42.

25. Clase CM, St Pierre MW, Churchill DN. Conversion between bromcresol green- and bromcresol purple-measured albumin in renal disease. Nephrol Dial Transplant. 2001;16:1925-9.

26. Kurland BF, Heagerty PJ. Directly parameterized regression conditioning on being alive: analysis of longitudinal data truncated by deaths. Biostatistics. 2005;6:241-58.

27. Wang Y. Smoothing Splines: Methods and Applications. New York: Champman and Hall; 2011.

28. RDC T. R: A language and environment for statistical computing. R Foundation for Statistical Computing. Vienna, Austria; 2009. 


\section{Chapter 9}

General discussion / summary 


\section{General discussion}

The major aim of this thesis was to describe and highlight the importance of dynamic time patterns in HD patients. It points out how cyclical and longitudinal trends are associated with both treatment-related complications and patient outcomes such as hospitalization and mortality. The study of time patterns may lead to increasing understanding of the pathophysiology of short- and long-term complications in dialysis patients; this kind of research may also have future therapeutic implications. The first part of this thesis dealt with oscillatory or cyclical trends (part A) and the second dealt with non-cyclical longitudinal trends (part B).

Part A consisted of two chapters dealing with two types of cycles: daily (circadian) and annual. In Chapter 2 we discussed the relevance of cyclical trends in BT in the pathogenesis of intra-dialytic hypotension (IDH), an important intra-dialytic morbid event that occurs in approximately $17 \%$ of dialysis treatments in $\mathrm{RRI}^{1,2}$. IDH is an important predictor of patient outcomes ${ }^{3,4}$. Usually, four main consequences of IDH are noted. First, it leads to discomfort to the patient. Second, it may have secondary effects after the patient leaves the clinic, such as falls and accidents ${ }^{5}$. Third, IDH may lead to structural damage to the heart, brain, and kidneys (reducing residual renal function). Fourth, IDH often means the clinic staff will give the patient extra saline to treat IDH. Additional saline may later be associated with more fluid gain ${ }^{6}$. Combined, this suggests that avoidance of hypotension may be associated with improved patient outcomes.

Traditional IDH is thought to occur for three main reasons during HD sessions. First, most dialysis treatments use ultrafiltration which results in a reduction in blood volume during dialysis. Second, most patients have impaired myocardial and vascular systems which do not properly respond to the process of dialysis ${ }^{7,8}$. Third, sympathetic vasoconstriction, a normal body response to fluid removal, may be impaired in dialysis patients due to autonomic neuropathy ${ }^{9}$. In addition, adequate vasoconstriction may be impaired because an increase in BT during dialysis causes a reflex vasodilation ${ }^{10}$. Similar findings are observed even in healthy subjects exposed to mild heat stress ${ }^{11}$.

An increase in BT during dialysis is known to occur, yet specific reasons for this increase are unknown. Some studies have suggested that increase in BT occurs because of an initial response to hypovolemia through "skin isolation" ${ }^{12}$. The importance of other factors was shown by the observation that BT still increased when administering dialysis without ultrafiltration ${ }^{13}$. Cooling of dialysate has been recommended to prevent or minimize the increase in BT during dialysis ${ }^{13,14}$. Some studies demonstrated that reductions in IDH may occur when using cool dialysate at temperatures of $35.0-35.5^{\circ} \mathrm{C}^{15,16}$. 
However, dialysate temperature per se is not the only reason for BT increase. In Chapter 2, we showed a relationship between the intradialytic increase in BT and the timing of the dialysis shift. This is likely due to the strong relation between pre-dialytic BT and the increase in BT during dialysis ${ }^{17}$. Indeed, patients studied in Chapter 2 had highest $B T$ in the evening shift and lowest in the morning shift, in line with the circadian rhythm in $\mathrm{BT}^{18}$.

While we did not have data on IDH on all patients studied in Chapter 2, a small subset of the data set suggested that IDH was more likely to occur in the morning when the increase in temperature was also highest. This was later confirmed in a study by Sands ${ }^{1}$. We were able to show that intradialytic changes in SBP were significantly different by time of day even after adjustment for patient demographics, comorbidities, and other treatment parameters.

In Chapter 2, we suggested that circadian rhythms may play an important role in the pathogenesis of IDH and thus may be related to patient outcomes. We demonstrated an association, but not necessarily a causal relationship, between circadian cycles, changes in BT, and frequency of IDH. In healthy subjects circadian increase in BT was shown to be associated with skin vasoconstriction ${ }^{19}$. It is unclear whether this happens in dialysis patients. Further studies should concentrate on demonstrating a cause and effect relationship between internal effects of BT per se and external factors, such as dialysate temperature. Additional analyses should also concentrate on whether dialysate temperature should be patient-customized depending on the time of day to account for these circadian rhythms. Insight into mortality by time of day is also important. 
In addition to circadian oscillatory patterns, cycles with other frequencies exist. Infradian cycles of annual duration or seasonal patterns were discussed in Chapter 3. In this chapter, we observed that in 15,056 HD patients treated in RRI, clear seasonal patterns in pre-dialysis SBP, pre-dialysis BT, inter-dialytic weight gains, and intradialytic changes in SBP and BT exist. Pre-dialysis SBP and inter-dialytic weight gain (IDWG) appeared highest in the winter months compared to summer. The findings on seasonal differences in physiological parameters were consistent with observations in non-uremic subjects ${ }^{20}$. However, it is likely that uremia-specific factors also play an important role in their pathogenesis. Given the strong relationship between volume overload and hypertension in dialysis patients, the increases in pre-dialysis SBP in winter were at least partly related to an increase in IDWG. Increase in IDWG is likely caused by increased fluid and salt intake during the winter months and reduced perspiration and fluid loss ${ }^{21,22}$. It is possible that other factors also influence the difference in blood pressure (BP) between the different seasons, such as adrenergic activity, vascular tone, vitamin D level, and protein consumption ${ }^{23-25}$. In Chapter 3, we demonstrated that potassium values were also higher in the winter months alluding to higher food intake in the winter. Given that these differences existed across all climate zones where RRI clinics are located, it remains to be studied which factors cause higher food consumption in the winter.

Our original finding in this study was that mortality in dialysis patients was significantly and consistently different between seasons, with highest mortality in the winter and the lowest in the summer months. Interestingly, mortality was significantly higher in the winter months in patients below the age of 75 years as compared to patients over 75 . This is in contrast to the non-dialysis population, where especially in the older population, mortality is associated with seasons ${ }^{26}$. It is likely that, in contrast to the general population, younger dialysis patients are frailer and more prone to failure of homeostatic mechanisms since their kidney disease affects different body systems, in analogy to processes observed during aging ${ }^{27-30}$.

Cardiovascular disease (CVD) mortality was also significantly higher in the winter. Somewhat surprisingly, infection-related mortality was not different between seasons, yet we found that neutrophil to lymphocyte ratio, a marker of inflammation, was indeed higher in the winter. While infection-related mortality was not higher in the winter months, it is possible that infections contributed to CVD mortality ${ }^{31}$.

Our key conclusion of this chapter was that seasonality in mortality and physiologic parameters should be taken into account in designs and analysis of epidemiologic studies. It remains to be seen whether mortality differences are a modifiable factor. Further, it needs to be studied whether differences in intra-dialytic changes in SBP are associated with increased frequency in IDH in the winter months. Dialysis treatment 
itself may need to be customized to account for differences in seasonal parameters. Additional analysis that looks into different hemispheres, varying climate zones, and even altitudes may shed additional light on variability in patient parameters seasonally.

In the second part of this thesis, we dealt with longitudinal but non-oscillatory patterns in HD patients and delineated how longitudinal patterns are associated with patient outcomes-both survival and hospitalization. Most current literature addresses how cross-sectional patient characteristics are related to outcomes but usually does not look at the relationship of the trends in patient parameters and outcomes $^{32,33}$. In this thesis we demonstrated that longitudinal patterns were associated with survival, and we examined both "forward-looking" and "backwardlooking" approaches.

The forward-looking approach starts from a certain point in time-such as initiation of dialysis-and tracks patients forward in time ${ }^{34,35}$. Trajectories of patient parameters are then characterized using linear mixed models or simple linear regression; other techniques such as variability of parameters over time can also be used. The resulting characterization of trajectory is used in the Cox proportional hazard models. This approach is one of the joint modelling methodologies that are used to model longitudinal and survival data described in the literature ${ }^{36-40}$. The result is a hazard risk of an outcome given a trend in a particular patient parameter.

The backward-looking approach selects a common end point for all patients and traces time backward from this end point. In other words, an event such as hospitalization or death is considered the end point for all patients, and all patients are then tracked back in time from the point of hospitalization in given increments, such as monthly, weekly, daily, etc. The trajectory of patient parameters before this event is also estimated using various techniques ${ }^{41,42}$. This trajectory is only used for descriptive purposes and not to predict hospitalization or death.

In Chapters 4 and 5, we utilized a forward-looking approach to follow incident dialysis patients over the first two years of their treatment. In Chapter 4, we demonstrated that both increases and decreases in SBP have a detrimental association with survival in incident HD patients in the first year of dialysis, irrespective of beginning BP value. Indeed, patients with seemingly normal pre-dialysis BP range of $<120 \mathrm{mmHg}$ and whose BP declines had worse outcomes than any other group. It was unclear from the data why an increase or decline in BP was associated with poorer outcomes. It can be hypothesized that increases in BP occur due to fluid overload while decreases in BP occur due to progressive myocardial failure ${ }^{43,44}$. These observations are consistent with the reverse epidemiology concept introduced earlier ${ }^{45}$, where survival of subjects 
who are at higher risk of death in the healthy population appear to have a lower risk of death in dialysis patients. Use of cardio-protective drugs appears to have an association with improved survival in both patients with increasing BP (antihypertensive aspect) and decreasing BP (cardio-protective aspect of cardioprotective drugs). Several sensitivity analyses were conducted to confirm these findings with the results consistently stating that the best survival is observed in patients with baseline BP of $150-180 \mathrm{mmHg}$ and whose BP remains stable (analysis of the patients who survived the first year and were followed into the second year, demonstrated nearly identical results).

In Chapter 5 we dealt with a similar forward-looking methodology but applied it to patients' BTs. In this study, we found that incident patients with BT increases or declines had a higher risk of mortality. The pathophysiologic mechanisms behind this observation need further study, although some suppositions may be made. And increase in BT is likely to be related to inflammation, while a decline in BT is likely a result of a reduction in metabolism. It has also been hypothesized that the body responds by reducing its core temperature when it is unable to respond to higher demands of fever in the presence of lower food intake ${ }^{46}$. This may also be related to non-thyroidal illnesses which are characterized by lowered BT and reduction in triiodothyronine (T3) levels ${ }^{47}$. In a recent study in dialysis patients, a relation between low T3 levels and mortality was observed ${ }^{48}$.

We acknowledge that the approach in Chapters 4 and $\mathbf{5}$ can be further improved by calculating the slope of BP or BT before the event. This would assure that every patient has a slope calculated over nearly the same number of treatments. Other marginal or time conditional Cox models can take this into account by calculating a slope of a variable on a pre-defined interval of time. This is an area that requires further studies.

Not only may the trajectories of parameters be important predictors of outcomes, but variability may also play a role. Recent analyses suggest that variability in serum sodium leads to poorer outcomes; similarly, a different study looked at variability in ultrafiltration rates (UFR) in relationship to outcomes ${ }^{49,50}$. Further studies that combine variability and direction of a parameter in relationship to outcome need to be undertaken.

In Chapters 6 and 8 we utilized a backward-looking approach. Compared to the forward-looking approach used in Chapters $\mathbf{4}$ and 5, we no longer tried to estimate the mortality risk based on the patients' trajectories; in these chapters we described what happened to patient parameters before an event such as hospitalization or death on a population level. The event that occurred at the end of an observation period became the common end point for all patients, with the patient parameters traced back in time. This type of analysis was different from the forward joint 
modelling approach discussed in Chapters 4 and 5. While this approach was not meant for predictive modelling, it was a descriptive methodology that allowed us to demonstrate what happened to patients' parameters before they died or were hospitalized on a population level ${ }^{32}$.

In Chapter 6, we found that many patient parameters follow distinct patterns before hospitalization. The intra-dialytic drop in systolic BP appeared to become smaller before hospital admission. The reduction in intra-dialytic systolic BP decline was most apparent in patients admitted for fluid overload.

Saline use during dialysis went up, suggesting more IDH episodes. Effective treatment time was shortened before hospitalization, likely due to clinical staff response to increased hypotension and the inability of the patients to tolerate the treatment.

Although conjectural, hemodynamic deterioration related to an emergent illness might reduce treatment tolerance, as indicated by a shortening of effective treatment time and the need for larger saline infusion volume, at a point when body cell mass might decline due to the emergent illness. A reduction in treatment tolerance during dialysis might further result in lower UFR, increased saline infusion, and reduction in treatment time ${ }^{51,52}$. A reduction in food and fluid intake due to loss of appetite might explain the reduction in IDWG.

Infections are likely causes of hospitalization in patients, and we found that BT increased in just three weeks before hospitalization in patients who were hospitalized for infections. In line with the increase in BT, the neutrophil to lymphocyte ratio went up significantly while albumin levels dropped. These observations would fit rationally as patients are more inflamed before they are hospitalized ${ }^{53,54}$. Hemoglobin also declined before hospitalization, which might be due to the effect of disease on the insufficient and often untimely response of additional erythropoiesis stimulating agents (ESA). Our study suggested that more frequent recording of the key laboratory parameters would assist in more refined modelling to predict patient hospitalizations.

It remains to be seen whether knowledge of these patterns can result in flagging of patients at increased risk of hospitalization and in potential therapeutic interventions. However, work by Dalrymple ${ }^{31}$ suggests that fluid overload is a modifiable factor in hospital admissions. Extending treatment time or offering additional treatments is another logical solution to this problem and the problem of IDH ${ }^{3}$.

Our analyses so far have dealt only with U.S. data. With the advent of faster computing and studies demonstrating the importance of longitudinal analytics, the need for more easily accessible data is imperative, particularly when integrated care 
becomes a reality in the U.S. Similar to other fields of medicine, dialysis data are kept only in country-specific registries. With the exception of the Dialysis Outcomes and Practice Pattern Study (DOPPS), no other global database on dialysis patients existed. The research from RRI on longitudinal dynamics of patient parameters led to the formation of the MONitoring Dialysis Outcomes (MONDO) initiative with a diverse group of dialysis providers. These providers agreed to combine and analyze the data from their respective electronic medical records (EMR) systems ${ }^{55-57}$, as discussed in chapter 7.

MONDO members have access to data from nearly 130,000 HD patients and close to 23 million HD treatments between 2000 and 2012. A distinctive characteristic of MONDO is that data is gathered at a patient- and (in most instances) treatment-level. This feature allowed the consortium to analyze individual patients longitudinally. MONDO contains HD patient data from eight dialysis providers worldwide, with 1,052 dialysis clinics on five continents. Current MONDO members include: Maastricht University Hospital (Netherlands), Hadassah Medical Center (Israel), Imperial College Renal and Transplant Center (U.K.), Kingston General Hospital / Queen's University (Canada), Fresenius Asia Pacific (five countries in the Asia Pacific region), Fresenius Medical Care Europe, Middle East, and Africa (17 countries), Fresenius Medical Care Latin America (five countries in South America), and the Renal Research Institute (U.S.A.).

Chapter 8 was the first product of the MONDO initiative; here we analyzed the dynamics of SBP, IDWG, serum albumin, and C-reactive protein (CRP) levels before death in the MONDO cohort. The choice of the parameters was based on the fact that they cover key areas of healthcare domains: nutritional, cardiovascular, and inflammatory. The method chosen was similar to that in Chapter 7 except that the common end point in Chapter 8 was mortality as opposed to hospitalization. Consistently, in all geographies studied, albumin, CRP, and SBP declined very similarly across all geographies and in different dialysis providers. IDWG declined before death in all areas studied but was much less pronounced in Southeast Asian patients.

In Chapter 8, we analyzed the trajectories of the aforementioned parameters by cause of death; irrespective of the cause of death, dynamics of albumin, IDWG, BP, and CRP were qualitatively comparable before death. Increases in CRP were similar irrespective of the cause of death. We also demonstrated that the majority of patients in each region died of cardiovascular disease, with the highest percentage in the U.S. (61\%) versus Southeast Asia with the lowest percentage (27\%). Neoplasms caused the lowest percentage of deaths in each region.

Given the large variability of background mortality and treatment patterns throughout the world ${ }^{58-61}$, this study confirmed that trends in patient parameters before death are 
remarkably similar. The main message for the clinical practitioner is that the temporal evolutions of albumin, BP, IDWG, and CPR are as important to follow as their absolute levels. This insight should also serve as a message for dialysis providers, since they may have to put information systems in place that allow physicians not only to follow absolute variable levels but also the dynamics.

Although these results are descriptive, understanding the factors that are associated with death may assist in the development of the predicative models that can be used in clinic settings to alert clinical staff to patients who are at great risk of death. Such advance warning systems may give physicians useful predictive information in their care of high-risk patients.

In summary, this thesis focused on pathophysiological and clinical implications of oscillatory and longitudinal patterns of several key clinical and laboratory parameters typically available in dialysis clinic settings. Analysis of worldwide data was also a crucial consideration in this thesis. Future studies should offer more insight into timedependent pathophysiological patterns in dialysis patients and might focus on the construction of early alert systems which provide clinical staff with a window of opportunity to intervene and avoid hospital admissions and deaths. 


\section{References}

1. Sands JJ, et al. Intradialytic Hypotension (IH): Variation in Dialysis Facilities in Annual Dialysis Conference. 2013. Seattle, USA.

2. Agarwal R. How can we prevent intradialytic hypotension? Curr Opin Nephrol Hypertens. 2012;21:593-9.

3. Kooman J, et al. EBPG guideline on haemodynamic instability. Nephrol Dial Transplant. 2007;22 Suppl 2:ii22-44.

4. Burton JO, et al., Hemodialysis-induced cardiac injury: determinants and associated outcomes. Clin J Am Soc Nephrol. 2009.4:914-20.

5. Aronow WS, Ahn C. Association of postprandial hypotension with incidence of falls, syncope, coronary events, stroke, and total mortality at 29-month follow-up in 499 older nursing home residents. J Am Geriatr Soc. 1997;45:1051-3.

6. Raimann J, et al. Consequences of overhydration and the need for dry weight assessment. Contrib Nephrol. 2008;161:99-107.

7. Parfrey PS, Foley RN. The clinical epidemiology of cardiac disease in chronic renal failure. J Am Soc Nephrol. 1999;10:1606-15.

8. van der Sande FM, et al., The hemodynamic effect of different ultrafiltration rates in patients with cardiac failure and patients without cardiac failure: comparison between isolated ultrafiltration and ultrafiltration with dialysis. Clin Nephrol. 1998;50:301-8.

9. Kooman JP, et al.,Role of the venous system in hemodynamics during ultrafiltration and bicarbonate dialysis. Kidney Int. 1992;42:718-26.

10. Rowell LB. Reflex control of the cutaneous vasculature. J Invest Dermatol, 1977;69:154-66.

11. Crandall CG, Gonzalez-Alonso J. Cardiovascular function in the heat-stressed human. Acta Physiol (Oxf). 2010;199:407-23.

12. Schneditz D, et al., Heat accumulation with relative blood volume decrease. Am J Kidney Dis. 2002; 40:777-82.

13. van der Sande FM, et al. Effect of ultrafiltration on thermal variables, skin temperature, skin blood flow, and energy expenditure during ultrapure hemodialysis. J Am Soc Nephrol. 2005;16:1824-31.

14. Mahida $\mathrm{BH}$, et al. Effect of cooled dialysate on serum catecholamines and blood pressure stability. Trans Am Soc Artif Intern Organs. 1983;29:384-9.

15. Selby, N.M. and C.W. Mclntyre, A systematic review of the clinical effects of reducing dialysate fluid temperature. Nephrol Dial Transplant. 2006;21:1883-98.

16. Maggiore $Q$, et al. Influence of blood temperature on vascular stability during hemodialysis and isolated ultrafiltration. Int J Artif Organs. 1985;8:175-8.

17. Fine A, Penner B. The protective effect of cool dialysate is dependent on patients' predialysis temperature. Am J Kidney Dis. 1996;28:262-5.

18. Kelly G. Body temperature variability (Part 1): a review of the history of body temperature and its variability due to site selection, biological rhythms, fitness, and aging. Altern Med Rev. 2006;11: 278-93.

19. Raymann RJ, Swaab DF, Van Someren EJ. Cutaneous warming promotes sleep onset. Am J Physiol Regul Integr Comp Physiol. 2005;288:R1589-97.

20. Alperovitch A, et al. Relationship between blood pressure and outdoor temperature in a large sample of elderly individuals: the Three-City study. Arch Intern Med. 2009;169:75-80.

21. Argiles A, Mourad G, Mion C. Seasonal changes in blood pressure in patients with end-stage renal disease treated with hemodialysis. N Engl J Med. 1998;339:1364-70.

22. Sposito M, Nieto FJ, Ventura JE. Seasonal variations of blood pressure and overhydration in patients on chronic hemodialysis. Am J Kidney Dis. 2000;35:812-8.

23. Argiles, A., et al., Blood pressure is correlated with vitamin $\mathrm{d}(3)$ serum levels in dialysis patients. Blood Purif. 2002;20:370-5.

24. Cheung AK, et al. Seasonal variations in clinical and laboratory variables among chronic hemodialysis patients. J Am Soc Nephrol. 2002;13:2345-52. 
25. van der Sande FM, Kooman JP, Leunissen KM. Clinical implications of seasonal variations in hemodialysis patients. Blood Purif. 2008;26:193-5.

26. Cold exposure and winter mortality from ischaemic heart disease, cerebrovascular disease, respiratory disease, and all causes in warm and cold regions of Europe. The Eurowinter Group. Lancet, 1997;349:1341-6.

27. Johansen KL, et al. Significance of frailty among dialysis patients. J Am Soc Nephrol, 2007;18:2960-7.

28. Kooman JP, et al. Renal replacement therapy in geriatric end-stage renal disease patients: a clinical approach. Blood Purif. 2012;33:171-6.

29. Lang PO, Michel JP, Zekry D. Frailty syndrome: a transitional state in a dynamic process. Gerontology. 2009;55:539-49.

30. Kooman JP, Broers NJ, Usvyat L, Thijssen S, van der Sande FM, Cornelis T, Levin NW, Leunissen KM, Kotanko P. Out of control: accelerated aging in uremia. Nephrol Dial Transplant. 2013;28:48-54.

31. Dalrymple LS, et al. Risk of cardiovascular events after infection-related hospitalizations in older patients on dialysis. Clin J Am Soc Nephrol. 6:1708-13.

32. Kotanko $\mathrm{P}$, et al. Temporal evolution of clinical parameters before death in dialysis patients: a new concept. Blood Purif, 2009;27:38-47.

33. Himmelfarb J, Kliger AS. End-stage renal disease measures of quality. Annu Rev Med. 2007;58: 387-99.

34. Gary Chan KC, Wang MC. Backward Estimation of Stochastic Processes with Failure Events as Time Origins. Ann Appl Stat. 2010;4:1602-20.

35. Kurland BF, Heagerty PJ. Directly parameterized regression conditioning on being alive: analysis of longitudinal data truncated by deaths. Biostatistics. 2005;6:241-458.

36. Hsieh F, Tseng YK, Wang JL. Joint modeling of survival and longitudinal data: likelihood approach revisited. Biometrics. 2006;62:1037-43.

37. Jacqmin-Gadda $H$, Thiebaut $R$, Dartigues JF. Joint modeling of quantitative longitudinal data and censored survival time. Rev Epidemiol Sante Publique. 2004;52:502-10.

38. Ratcliffe SJ, Guo W, Ten Have TR. Joint modeling of longitudinal and survival data via a common frailty. Biometrics. 2004;60:892-9.

39. Song X, Wang CY. Semiparametric approaches for joint modeling of longitudinal and survival data with time-varying coefficients. Biometrics. 2008;64:557-66.

40. Tapsoba Jde D, Lee SM, Wang CY. Joint modeling of survival time and longitudinal data with subjectspecific changepoints in the covariates. Stat Med. 2011;30:232-49.

41. RDC, T, R: A language and environment for statistical computing. R Foundation for Statistical Computing. 2009, Vienna: Austria.

42. Wang Y. Smoothing Splines: Methods and Applications. 2011, New York: Champman and Hall.

43. Kalantar-Zadeh K, et al. Risk factor paradox in wasting diseases. Curr Opin Clin Nutr Metab Care. 2007;10:433-42.

44. Wizemann $\mathrm{V}$, et al. The mortality risk of overhydration in haemodialysis patients. Nephrol Dial Transplant. 2009;24:1574-9.

45. Kalantar-Zadeh K. What is so bad about reverse epidemiology anyway? Semin Dial. 2007;20:593-601.

46. Romanovsky AA, Szekely M. Fever and hypothermia: two adaptive thermoregulatory responses to systemic inflammation. Med Hypotheses. 1998;50:219-26.

47. Koenig RJ. Modeling the nonthyroidal illness syndrome. Curr Opin Endocrinol Diabetes Obes. 2008; 15:466-9.

48. Zoccali C, et al. Low triiodothyronine and survival in end-stage renal disease. Kidney Int. 2006;70: 523-8.

49. Raimann J, et al. Relationship between variability of pre dialysis serum sodium and mortality risk in incident hemodialysis patients in ERA-EDTA. 2012. Paris.

50. Usvyat LA, and M. consortium. Variability of ultrafiltration rate is associated with poor outcomes in US and Asian Pacific dialysis patients. in ASN. 2013. San Diego.

51. Coyne DW, et al. Impaired delivery of hemodialysis prescriptions: an analysis of causes and an approach to evaluation. J Am Soc Nephrol. 1997;8:1315-8. 
52. Knoll GA, et al. A randomized, controlled trial of albumin versus saline for the treatment of intradialytic hypotension. J Am Soc Nephrol. 2004;15:487-92.

53. Turkmen $\mathrm{K}$, et al. The relationship between neutrophil-to-lymphocyte ratio and inflammation in endstage renal disease patients. Ren Fail. 2012;34:155-9.

54. Thijssen S, et al. Determinants of serum albumin concentration analyzed in a large cohort of patients on maintenance hemodialysis. J Ren Nutr. 2007;17:70-4.

55. Obama administration calls for electronic health records by 2015, in USA Today.

56. Jager KJ, Zoccali C. Clinical databases and the QUEST initiative. Blood Purif. 2007;25:219-20.

57. Krishnan M, Wilfehrt HM, Lacson E Jr. In Data We Trust: The Role and Utility of Dialysis Provider Databases in the Policy Process. Clin J Am Soc Nephrol. 2012;7:1891-6.

58. Yoshino $\mathrm{M}$, et al. International differences in dialysis mortality reflect background general population atherosclerotic cardiovascular mortality. J Am Soc Nephrol. 2006;17:3510-9.

59. Pisoni RL, Greenwood RN. Selected lessons learned from the Dialysis Outcomes and Practice Patterns Study (DOPPS). Contrib Nephrol. 2005;149:58-68.

60. Pifer TB, et al. Mortality risk in hemodialysis patients and changes in nutritional indicators: DOPPS. Kidney Int. 2002;62:2238-45.

61. Goodkin DA, et al. Association of comorbid conditions and mortality in hemodialysis patients in Europe, Japan, and the United States: the Dialysis Outcomes and Practice Patterns Study (DOPPS). J Am Soc Nephrol. 2003;14:3270-7. 
Nederlandse samenvatting en discussie 


\section{Nederlandse samenvatting en discussie}

Hemodialyse (HD) is de meest gebruikte behandeling bij patiënten met een eindstadium nierfalen. Bij patiënten met een eindstadium nierfalen zijn de functies van de nier, welke onder meer bestaan uit verwijdering van afvalproducten en overtollig water- en zout, en uit de instandhouding van een normale mineralenbalans verstoord. Een deel van deze functies kan worden overgenomen door HD, waarbij uitwisseling plaatsvindt tussen bloed van de patiënt en een gezuiverde waterige oplossing, welke gescheiden zijn door een halfdoorlaatbare membraan.

HD wordt in het algemeen 3x/week uitgevoerd. waarbij onder meer afvalproducten worden verwijderd en overtollige water en zout wordt verwijderd. Het feit dat deze behandeling slechts gedurende een relatief kort moment wordt uitgevoerd is in tegenstelling met de continue werking van de normale nier. Dit kan leiden tot ophoping van onder meer water en zout (gereflecteerd door een toename van het gewicht) en afvalproducten tussen 2 dialysebehandelingen in. Ook de snelle wisselingen tijdens de behandeling kan problemen voor de patiënt met zich meebrengen, zoals perioden van lage bloeddruk. Naast deze korte-termijn complicaties treden ook frequent lange-termijn complicaties bij dialysepatiënten op. Hierbij kunnen onder meer een verhoogd optreden van hart- en vaatziekten, waarbij onder meer een onvoldoende verwijdering van water- en zout en hieraan gerelateerde verhoging van de bloeddruk een rol speelt, alsmede een verhoogd voorkomen van ondervoeding en infectieuze complicaties genoemd worden. Het verhoogd voorkomen van deze complicaties leidt er mede toe dat de overleving bij dialysepatiënten verminderd is terwijl het aantal ziekenhuisopnames verhoogd is ten opzichte van de algemene bevolking. Er zijn reeds vele onderzoeken verricht naar de oorzaken van deze complicaties bij dialysepatiënten. Een factor welke tot heden nog weinig belicht is, is de rol van biologische tijdspatronen bij het optreden van korte- en lange termijn complicaties bij dialysepatiënten.

Het doel van dit proefschrift is het beschrijven van de fysiologische en klinische implicaties van cyclische en longitudinale tijdspatronen bij HD patiënten. Het proefschrift geeft aan op welke manier (veranderingen in) deze tijdspatronen geassocieerd zijn met zowel aan de dialysebehandeling gerelateerde korte-termijn complicaties, als aan lange-termijn complicaties bij dialysepatiënten.

Het proefschrift is in twee delen ingedeeld, in het eerste deel is onderzoek gedaan naar de implicaties van oscillatoire of cyclische tijdspatronen, en in het tweede deel is onderzoek gedaan naar de longitudinale trends.

In het eerste deel worden de implicaties van 2 cyclische tijdspatronen bestudeerd: dagelijkse (circadiane) cycli en jaarlijkse cycli. In Hoofdstuk 2 werd de relatie tussen circadiane veranderingen in de lichaamstemperatuur en het optreden van bloeddrukdalingen tijdens dialyse (intradialytische hypotensie, IDH) beschreven. IDH, een belangrijke complicatie welke frequent optreedt tijdens een dialysebehandeling, 
kan meerdere gevolgen hebben. Ten eerste Het geeft een gevoel van onbehagen bij de patiënt, en kan het leiden tot complicaties aan andere organen zoals hart en hersenen. Bij IDH is soms behandeling met een zoutinfuus noodzakelijk om de bloeddrukdaling te behandelen hetgeen kan leiden tot onvoldoende verwijdering van water- en zout tijdens de dialysebehandeling. Dit kan bijdragen tot ophoping van vocht in het lichaam. Het voorkomen van IDH is dus een belangrijk doel.

Drie fenomenen zijn met name van belang van belang bij het ontstaan van IDH. Ten eerste, tijdens de meeste dialyse behandelingen wordt er ook vocht ontrokken (ultrafiltratie) met als gevolg een daling in het circulerend bloedvolume tijdens dialyse. Ten tweede zijn bij dialysepatiënten vaak afwijkingen in de hartfunctie aanwezig, waardoor de gevoeligheid voor veranderingen in het bloedvolume toeneemt. Ten derde kan tijdens dialyse de normale reactie van het vaatsysteem tijdens een daling in het bloedvolume, welke bestaat uit een constrictie (samentrekking) van de kleine slagaders en venen, gestoord zijn. Eerder onderzoek heeft gewezen dat deze abnormale reactie te wijten is aan een stijging van de lichaamstemperatuur tijdens dialyse, waardoor de bloedvaten in de huid open blijven staan.

Het is bekend dat de lichaamstemperatuur tijdens dialyse stijgt, maar de oorzaken hiervan zijn tot op heden nog niet volledig bekend. Naast een effect van de instelling van de temperatuur van de dialysevloeistof hebben diverse onderzoeken gesuggereerd dat de stijging in lichaamstemperatuur een gevolg kan zijn van hypovolemie (afname van vocht in de bloedvaten) tijdens het begin van de dialysebehandeling, leidend tot initiële samentrekking van de bloedvaten en "isolatie" van de huid. Dit wordt gevolgd door een stijging van de lichaamstemperatuur en een reflexmatige verwijding van de bloedvaten in de huid. Het belang van andere oorzaken werd aangetoond in onderzoek waarbij de lichaamstemperatuur ook bleek te stijgen tijdens HD zonder ontwatering.

Eerdere onderzoeken toonden aan dat ook de temperatuur van de dialysevloeistof een belangrijke rol speelt bij de temperatuursveranderingen tijdens dialyse. Dit heeft geleid tot de aanbeveling om de temperatuur van de dialysevloeistof bij patiënten met frequente episoden van IDH te verlagen, om zodoende de stijging van de lichaamstemperatuur tijdens dialyse te verminderen. Meerdere onderzoeken hebben inderdaad aangetoond dat een vermindering in het aantal bloeddrukdalingen tijdens dialyse bereikt kan worden door gebruik van zogenaamd "koud" dialysaat met een temperatuur van $35.0-35.5^{\circ} \mathrm{C}$.

De temperatuur van het dialysaat en de vochtverwijdering tijdens dialyse zijn echter niet de enige reden voor de stijging van de lichaamstemperatuur. De achtergrond van deze andere oorzaken zijn echter nog niet goed bekend. Wel valt het tijdstip van de dialysebehandeling vaak samen met de tijden waarop de lichaamstemperatuur tijdens het normale circadiane temperatuursritme ook stijgt. Indien het normale circadiane ritme ook een rol zou spelen bij de temperatuursstijgingen tijdens dialyse zou 
verwacht kunnen worden dat de stijging in lichaamstemperatuur tijdens dialyse het hoogste zou zijn in de ochtend en het laagste in de middag, wanneer ook de stijging in het normale circadiane ritme het meest uitgesproken is.

In Hoofdstuk 2 hebben we aangetoond dat er een relatie leek te zijn tussen de stijging van de lichaamstemperatuur tijdens dialyse en het tijdstip van de dag waarop er wordt gedialyseerd. De veranderingen waren het meest uitgesproken in patiënten welke in de ochtend en de middag werden gedialyseerd, wanneer ook de normale circadiane temperatuurstijging het meest uitgesproken is, en het minst uitgesproken in patiënten welke's avonds werden gedialyseerd. Tevens leken er ook meer episoden van IDH te zijn bij die groep van patiënten die in de ochtend dialyseerden en waarbij er ook en duidelijke stijging in de lichaamstemperatuur tijdens dialyse was. Uit deze observaties kunnen we echter niet definitief concluderen dat de stijging van de lichaamstemperatuur tijdens dialyse, welke gerelateerd is aan het normale circadiane ritme, ook inderdaad een bijkomende oorzaak is voor het optreden van bloeddrukstijgingen tijdens dialyse. Een mogelijke implicatie van deze studie is dat bij de instelling van de temperatuur van de dialysevloeistof rekening gehouden moet worden met de trends in de lichaamstemperatuur van de patiënt, en zodoende individueel ingesteld moet worden. Verder onderzoek zal het klinische belang van de uitkomsten in Hoofdstuk 2 nader moeten aantonen.

Naast circadiane ritmen (met een periode van ongeveer 24 uur), bestaan er ook cycli met een andere periode, te weten infradiane ritmes (ritmes met een periode langer dan 24 uur). Een belangrijk infradiaan ritme is het optreden van de seizoenen tijdens de loop van het jaar. Eerdere onderzoeken hebben bij dialyse patiënten seizoen gerelateerde patronen gevonden in belangrijke parameters zoals bloeddruk en de gewichtstoenames tussen 2 dialysebehandelingen in. Dit betroffen echter met name studies bij een beperkte groep patiënten. Ook werd in deze studies niet gekeken naar de relatie tussen seizoen en mortaliteit (sterfte) bij dialyse patiënten. Deze patronen werden in Hoofdstuk 3 nader bestudeerd In dit hoofdstuk zijn 15,056 HD patiënten die behandeld werden in het RRI beschreven. Er waren duidelijke seizoen patronen aanwezig in de systolische bloeddruk, de gewichtsverandering tussen de dialyse behandelingen (IDWG, interdialytic weight gain), en de bloeddrukveranderingen tijdens de dialyse behandeling. De systolische bloeddruk voor dialyse en de IDWG waren het grootst in de winter vergeleken met de zomer. Gezien de sterke relatie tussen overvulling (teveel aan vocht in het lichaam) en hypertensie (hoge bloeddruk) bij dialyse patiënten, kan de stijging in de bloeddruk voor dialyse waarschijnlijk gedeeltelijk verklaard worden door een toename in IDWG. De toename in IDWG in de winter is waarschijnlijk veroorzaakt door een toename in inname van water en zout en een afname in transpiratie en vochtverlies in dit seizoen. Mogelijk kunnen ook andere factoren het verschil in bloeddruk tussen de verschillende seizoenen mede verklaren, zoals de hoogte van vitamine D spiegels, maar deze zijn in dit hoofdstuk niet bestudeerd. 
De oorspronkelijke bevinding van dit onderzoek was dat de mortaliteit bij dialysepatiënten significant en consistent verschilde tussen de verschillende seizoenen, met de hoogste mortaliteit in de wintermaanden en de laagste in de zomermaanden. Alhoewel een verschil in sterfte tussen seizoenen ook gevonden is bij met name oudere personen in de algemene populatie, is een opvallende bevinding van dit hoofdstuk dat ook bij jongere dialyse patiënten de seizoen gerelateerde mortaliteit verhoogd is. Mogelijk zijn, in tegenstelling tot de algemene populatie, jongere dialyse patiënten gevoeliger voor externe factoren door het falen van normale homeostatische (processen welke het lichaamsevenwicht in stand houden) mechanismen. Dit zou verklaard kunnen worden doordat het verlies aan nierfunctie verschillende systemen in het lichaam beïnvloedt, in analogie aan processen die optreden bij het ouder worden.

De mortaliteit door cardiovasculaire ziekten was ook significant hoger in de wintermaanden. Enigszins verassend was het feit dat overlijden door infecties niet seizoensafhankelijk was. Wel was de verhouding neutrofielen/lymfocyten, een maat voor ontsteking, hoger in de wintermaanden. Alhoewel de aan ontstekingen gerelateerde mortaliteit niet hoger was in de wintermaanden, is het mogelijk dat ontstekingen en infecties hebben bijgedragen aan het overlijden door cardiovasculaire ziekten, een fenomeen dat eerder in andere studies is aangetoond.

De belangrijkste conclusies van dit hoofdstuk waren dat seizoensafhankelijke factoren als overlijden en andere fysiologische parameters mee genomen moeten worden bij het ontwerpen en analyseren van epidemiologische onderzoeken. Nadere studies moeten uitwijzen of seizoensgerelateerde verschillen in overlijden te beïnvloeden zijn. In het tweede deel, deel B, van dit proefschrift werden longitudinale (niet cyclische patronen welke zich in de loop der tijd voordoen) patronen bij HD patiënten onderzocht en werd aangeven hoe longitudinale patronen in belangrijke klinische en laboratoriumbepalingen ("risicofactoren") geassocieerd waren met patiënt uitkomsten, zowel ten aanzien van overleving als hospitalisatie. In de literatuur werd de relatie tussen risicofactoren en patiëntuitkomsten werd, tot op heden, de risicofactor meestal slechts op een tijdspunt (cross-sectioneel) bestudeerd, en werd in het algemeen niet naar de relatie tussen trends in risicofactoren en patiëntuitkomsten gekeken. In dit proefschrift hebben we kunnen aantonen dat longitudinale patronen geassocieerd waren met overleving, zowel bij het gebruiken van een zogenaamde "vooruitkijkende" (forward-looking) als "terugkijkende" (backward-looking) benadering.

De "vooruitkijkende" benadering start op een moment in de tijd, bijvoorbeeld start van de nierfunctievervanging (dialyse), en volgt de patiënt in de tijd. Trajecten van patiënten parameters worden daarna gekarakteriseerd door gebruik te maken van statische modellen zoals "lineaire mixed modellen" of "een lineaire regressie". Het resultaat is een kans op een bepaalde uitkomst gegeven de trend in een bepaalde patiënt parameter. 
De "terugkijkende" benadering kiest een bepaalde eindpunt bij alle patiënten en kijkt dan terug in de tijd vanaf dit eindpunt. Met andere woorden, een gebeurtenis zoals bijvoorbeeld hospitalisatie of overlijden wordt dan beschouwd als het eindpunt voor alle patiënten, en de patiënten worden vanaf dit punt terug vervolgd in de tijd in gegeven perioden zoals bijvoorbeeld maanden, weken, dagen, enzovoort.

In Hoofdstukken 4 en 5, werd gebruikt gemaakt van de "vooruitkijkende"benadering om nieuwe dialyse patiënten de eerste 2 jaar na start van hun behandeling te volgen. In Hoofdstuk 4 werd aangetoond dat zowel een toename als een daling in de systolische bloeddruk (bovendruk) voor dialyse een ongunstige associatie hadden met overleving, onafhankelijk van de hoogte van de bloeddruk bij de start van hun nierfunctievervangende therapie. Echter, met name patiënten met een systolische bloeddruk (bovendruk) voor de dialyse beneden $120 \mathrm{mmHg}$ hadden een slechtere uitkomst dan enige andere groep.

Vanuit de data kan niet met zekerheid gesteld worden waarom een toename of daling in de bloeddruk geassocieerd was met een slechtere uitkomst. Het zou verondersteld kunnen worden dat een toename in de bloeddruk veroorzaakt werd door overvulling met vocht en een daling van de bloeddruk door progressief hartfalen. Het feit dat een daling van de bloeddruk gerelateerd bleek aan een verhoogde mortaliteit is consistent met het concept van de zogenaamde "reverse epidemiology" waar risicofactoren welke bij de algemene populatie gerelateerd zijn aan een hogere kans op overlijden (zoals een daling van de bloeddruk) bij dialyse patiënten juist soms een verlaagde kans op overlijden geven. Vanzelfsprekend mag hieruit niet geconcludeerd worden dat een adequate bloeddrukbehandeling bij dialysepatiënten niet van belang is, alleen dat er bij deze populatie onderliggende processen een rol kunnen spelen, welke de normale associatie tussen risicofactoren en patiëntuitkomsten kunnen verstoren.

In Hoofdstuk 5 werd de "vooruitkijkende" benadering methode toegepast op de lichaamstemperatuur van de patiënt. In dit onderzoek vonden we dat wanneer in nieuwe dialysepatiënten de lichaamstemperatuur steeg of daalde een hoger risico op overlijden hadden. Alhoewel het mechanisme achter deze observatie nader onderzocht dient te worden zou gehypothetiseerd kunnen worden dat een stijging van de lichaamstemperatuur wordt waarschijnlijk veroorzaakt door een ontsteking, een daling door een afname in het metabolisme (stofwisseling). Eerdere studies hebben gesuggereerd dat het lichaam kan reageren met een daling in de lichaamstemperatuur wanneer het niet in staat is te voldoen aan de hogere eisen van koorts door een verminderde lichaamsreserve. Of hierbij ook daling in de schildklierhormoon spiegel (tri-iodothyronine, T3), welke vaker gevonden wordt bij ernstig zieke patiënten een rol speelt zal nader onderzoek moeten uitwijzen.

In Hoofdstukken 6 en $\mathbf{8}$ werd de "terugkijkende" benadering toegepast. Het verschil met "vooruitkijkende" benadering welke toegepast is in de Hoofdstukken $\mathbf{4}$ en $\mathbf{5}$ is dat nu niet langer het risico op overlijden geschat is op diverse ontwikkelingen bij de patiënt; in de hoofdstukken 6 en 8 is beschreven wat er gebeurde met patiënt 
parameters voor bijvoorbeeld opname of overlijden. De gebeurtenis welke optrad aan het einde van een observatie werd het gemeenschappelijke eindpunt voor alle patiënten met de parameters van de patiënten terug vervolgd in de tijd. Deze wijze van analyse is duidelijk verschillend van het model gebruikt in Hoofdstukken 4 en 5. Hoewel het doel van dit model niet was om voorspellingen te kunnen maken, bood het toch de mogelijkheid om te beschrijven wat er gebeurde met de parameters van de patiënten voordat zij werden opgenomen of overleden.

In Hoofdstuk 6 vonden we dat meerdere parameters een duidelijk patroon volgden voordat de patiënten opgenomen werden (hospitalisatie). Dit betrof onder meer een daling in het hemoglobine gehalte (rode bloedcelgehalte), een daling van het serum albumine, een stijging van het neutrofielen/lymfocytengehalte en een stijging in de lichaamstemperatuur. Deze observaties zijn waarschijnlijk te verklaren door een verhoogde ontstekingstoestand voor hospitalisatie. In dit hoofdstuk werden ook een aantal opvallende beïnvloeding gedaan, zoals een afname van de daling van de systolische bloeddruk tijdens dialyse en een daling in de IDWG in de periode voor de hospitalisatie. Mogelijk is dit te wijten aan enerzijds een afname in de eetlust, en hieraan gerelateerd de water- en zoutinname, en anderzijds aan het feit dat het streefgewicht (het gewicht dat bereikt moet worden aan het eind van de dialyse) nog niet naar beneden is bijgesteld ondanks een afname van de spier- en vetmassa tijdens de ziekteperiode. Hierbij zou ook een verminderde tolerantie voor vochtverwijdering tijdens de dialyse een rol kunnen spelen. Inderdaad werd in de periode voor hospitalisatie een afname van de effectieve dialysetijd en een toename van het de zoutinfusie tijdens dialyse vastgesteld. Door al deze factoren zou ten onrechte te weinig water en zout verwijderd worden, waardoor patiënten ongemerkt "overvuld" zouden kunnen raken.

Hospitalisatie bleek een belangrijke risicofactor voor overlijden te zijn. Met name in de periode voor overlijden werd een zeer snelle toename van het aantal ziekenhuisopnames gevonden.

Nader onderzoek moet uitwijzen of kennis van bovenbeschreven patronen kan bijdragen in het ontwikkelen van een waarschuwingsysteem voor die patiënten die een toegenomen risico op opname hebben en in eventuele potentiële therapeutische opties. Eerder onderzoek toonde wel aan dat overvulling een mogelijk corrigeerbare factor is om ziekenhuisopnamen te voorkomen. Het verlengen van de behandeltijd of het aanbieden van meer behandelingen is een logische oplossing om dit probleem en bloeddrukdalingen tijdens dialyse te voorkomen.

Tot zover zijn de analyses gebaseerd op de Amerikaanse data. Met de komst van snellere computersystemen en onderzoeken die het belang van longitudinaal onderzoek aantonen is het noodzakelijk om meer en gemakkelijke toegangbare data te verkrijgen. Ook is het van belang om te bestuderen of de gevonden patronen kunnen worden geëxtrapoleerd naar andere landen. Met uitzondering van de "Dialysis Outcomes and Practice Pattern Study (DOPPS)" bestaat er wereldwijd geen andere 
database waarin risicofactoren en patiëntuitkomsten worden bestudeerd. Het onderzoek van het RRI in New York naar de dynamiek van longitudinale parameters van dialyse patiënten heeft mede geleid tot de oprichting van het "MONitoring Dialysis Outcomes (MONDO)" initiatief, een wereldwijd initiatief bestaande uit een groep van aanbieders van dialyse. Deze aanbieders zijn overeengekomen om de data van hun elektronische medische gegevens (electronic medical records (EMR)) te combineren en te analyseren zoals in hoofdstuk 7 van dit proefschrift is beschreven.

De leden van de MONDO groep hebben toegang tot de data van ongeveer 130,000 HD patiënten en bijna 23 miljoen HD behandelingen uitgevoerd tussen 2000 en 2012. Een onderscheidende eigenschap van MONDO is dat de data verzameld worden op patiënt- en (in de meeste gevallen) behandelingniveau. Door deze eigenschap is het consortium in staat de individuele patiënten longitudinaal te analyseren. MONDO bevat de gegevens van hemodialyse patiënten van 8 wereldwijde aanbieders van dialyse met in totaal 1052 dialyse klinieken verdeeld over 5 continenten. De leden van de huidige MONDO groep zijn; Maastricht University Medical Center (Nederland), Hadassah Medical Center (Israel), Imperial College Renal and Transplant Center (Engeland), Kingston General Hospital / Queen's University (Canada), Fresenius Asia Pacific (5 landen in de Azië Pasific regio), Fresenius Medical Care Europa, Midden Oosten, en Afrika (17 landen ), Fresenius Medical Care Latijns Amerika (5 landen in Zuid Amerika), en het RRI (Verenigde Staten).

In Hoofdstuk 8 werden in het MONDO cohort de trends geanalyseerd van de systolische bloeddruk, IDWG, serum albumine, en het C-reactieve proteïne (CRP) voor overlijden. De keuze van deze parameters is gebaseerd op het feit dat dit risicofactoren zijn met betrekking tot belangrijke aspecten welke de overleving van dialysepatiënten beïnvloeden, te weten hart- en vaatziekten, voedingstoestand en ontsteking. De methode voor analyse welke gebruikt werd was dezelfde als beschreven in Hoofdstuk 6 met uitzondering van het gemeenschappelijke eindpunt, deze was in Hoofdstuk 6 ziekenhuisopname en in Hoofdstuk 8 overlijden. In alle onderzochte geografische regio's was er een consistente verandering in albumine, $\mathrm{CRP}$, en systolische bloeddruk, nagenoeg gelijkwaardig bij alle aanbieders van dialyse en regio's. De toename in gewicht tussen de dialysebehandelingen nam af voor overlijden in alle onderzochte regio's, maar was het minst uitgesproken in Zuidoost Azië.

In Hoofdstuk 8, werden de ontwikkelingen van de eerdergenoemde parameters naar oorzaak van overlijden geanalyseerd; onafhankelijk van de oorzaak van overlijden waren de veranderingen in albumine, IDWG, bloeddruk, en CRP vergelijkbaar. Onafhankelijk van de oorzaak van overlijden was de stijging van CRP vergelijkbaar. We hebben ook aangetoond dat het hoogste aantal patiënten in elke regio overleed aan hart- en vaatziekten, met het hoogste percentage in de Verenigde Staten (61\%). 
Gegeven de grote variabiliteit in de onderliggende mortaliteit en behandelingen over de hele wereld, toonden de resultaten van dit onderzoek dat de trends in patiënten parameters voor overlijden opmerkelijk vergelijkbaar zijn. De belangrijkste boodschap voor de praktiserend clinicus zijn dat ook trends in belangrijke parameters zoals albumine, bloeddruk, IDWG, en CRP vitale informatie kunnen bevatten, naast de absolute waarde van de parameters. Dit inzicht is van groot belang voor aanbieders van dialyse, om informatiesystemen te gebruiken die niet alleen de absolute waarden laten zien maar ook de veranderingen in de tijd daarin.

Hoewel de resultaten beschrijvend zijn is het waarschijnlijk van groot belang om te begrijpen welke factoren geassocieerd zijn met overlijden, om te komen tot een voorspellend model waarvan gebruik gemaakt kan worden in de kliniek voor die patiënten die een hoger risico op overlijden hebben. Clinici kunnen zo'n waarschuwingssysteem gebruiken bij de zorg en behandeling van hoogrisico patiënten. Voor het ontwikkelen van een dergelijk model, welke toepasbaar is voor een individuele patiënt, is echter nog veel onderzoek nodig.

Concluderend, dit proefschrift heeft zich gefocusseerd op de pathofysiologische en klinische gevolgen van oscillatoire en longitudinale patronen van verschillende belangrijke (sleutel) klinische en laboratorium parameters die specifiek zijn voor een dialyse behandeling. Analyse van wereldwijde data kan hierbij een zeer belangrijke rol spelen. Verder onderzoek moet meer inzicht in de tijdsafhankelijke pathofysiologische patronen bij dialyse patiënten verschaffen en zou zich kunnen richten op de ontwikkeling van een vroegtijdig waarschuwingssysteem waarmee de klinische staf de mogelijkheid heeft om voortijdig in te grijpen en daarmee opname en overlijden te voorkomen. 
Acknowledgement / Dankwoord 


\section{Acknowledgement / Dankwoord}

This thesis is a product of many people who have helped and inspired me over the years in various capacities, both directly and indirectly. It would have never transpired if it were not for the endless and tireless dedication of my advisors from the University of Maastricht, RRI, and Fresenius medical teams, but also the support of my family. The guidance I have received from RRI fellows, University collaborators in the US and internationally, our MONDO members, and others at Fresenius was essential to making this happen. I sincerely feel that my contribution to this work is a small piece of a notably larger effort of many.

My advisors, Dr. Frank van der Sande and Dr. Jeroen Kooman, are incredible individuals who have travelled indefatigably to help and guide me through the dissertation process. On multiple occasions they worked very late hours, most certainly giving up their personal time with their families and friends. I am beyond grateful to them for what they have done and the dedication they put into this thesis. They have not only helped me with content, they have guided me through the labyrinth of Dutch and University traditions. I can never repay my debt to them for their kindness and generosity.

I have to express my deepest and most sincere appreciation to Dr. Nathan Levin, the first nephrologist I ever met, for becoming my first teacher in the field of dialysis. Many years ago, he caused a change in my future career path by allowing me access to RRI's clinical database. Without his initial contacts with the University of Maastricht, this PhD work might not have happened. Over many years of working together, I have learned about nephrology from him and to appreciate the importance of what we do.

Dr. Peter Kotanko is not only an amazing physician, but also a wonderful individual who has tirelessly explained medicine to me. He put a "face" to the numbers and made me realize that I can help patients' well-being. He trusted me to work independently with our many global MONDO partners in creating the largest dialysis database known worldwide, which has resulted in so many unforeseen and wonderful outcomes. He further strengthened relationships with the University of Maastricht by signing a more formal collaboration agreement that spearheaded multiple joint publications as well as this and other PhD efforts. I am extremely grateful and indebted to him for all that he has done, which ultimately resulted in this work.

I am also obliged to Prof. K.M. Leunnissen, who agreed to accept me into this PhD program. He allowed for such an amazing collaboration between the two centers and provided his input into my work. I am honored and grateful for all that he has done. 
I am most appreciative to my Degree Committee members Prof. M.P. van DieijenVisser, Prof. A. Schols, Prof. C.D.A. Stehouwer, Prof. P.M. ter Wee. I appreciate their willingness to wait for my many reviews and edits to this thesis.

Norma Ofsthun was able to give me her blessing many years ago to do something unprecedented: gain access to the FMCNA clinical data warehouse. That crucial decision also had unprecedented repercussions for what I will end up dedicating my career to. Now that we are colleagues in the same department, I am indebted to her for that far-reaching decision.

While I have met Dr. Frank Maddux only recently, I appreciate that he allowed me to finish this work and to dedicate as much time as necessary to do so. His decision to alter my career path is something for which I am very thankful.

I am also thankful to Dr. Paul Balter for his insight not only into medical aspects of dialysis, but also his acumen concerning the operational understanding of how clinics run. In line with this, I am grateful to all the nursing staff members who make dialysis clinics what they are. I'm also grateful to the president of RRI and the long list of nurses and technical and clinical staff I work with, and I'd like to highlight some including Paul Zabetakis, Terry Sullivan, Nancy Ginsberg, Lisa Pacelli, Patrice Taylor, Chris Hanrahan, Seth Johnson, and Rob Levin.

I'd like to express my appreciation to Mary Carter and John Callegari, who have often guided my professional decisions at RRI as well as Dr. Stephan Thijssen and Dr. Jochen Raimann, who consistently amaze me by their ability to combine medicine and epidemiology.

I am also thankful for many statisticians and epidemiologists with whom I have the pleasure to work and who both directly and indirectly guided me through this process, particularly Linda Ficociello and Yuedong Wang, professor of Biostatistics at the University of Santa Barbara.

Professionally, there are many others to whom l'd like to express my gratitude including Bruce Brown, who steered me toward applying for a job with RRI, Lisa Rockhill, John Keene, and Rachel Weinberger.

I would like to thank Tiny Wouters, Maastricht University Medical Center, for preparing the manuscript and lay-out. 
There are many other individuals to whom I am grateful, especially those who ended up traveling anywhere from 20 to $6,000 \mathrm{~km}$ to be here on the day of my defense. While I was only expecting this to be an academic affair, it ended up covering several continents, so I am sincerely thankful to everyone who was able to make it. This thesis covers a wide geographical area, just like the people who took part in this defense.

On a more personal and family note, I am so grateful to my partner Chris! He has spent so many days, evenings, and weekends while I was either traveling or was home working very late evenings. I can honestly say that without his personal support, encouragement, and love, I would have never accomplished this (and I have to admit, he did proofread this thesis). I am very, very lucky to have him. I also am very grateful to his parents, Debbi and Steve, who have allowed me to take many hours off from our house renovations!

I most certainly have to thank my parents, Abram and Yelena. My dad got his PhD when he was 40 , and I remember that day very well even though I was only nine. His continued drive for more education was thoroughly instilled into my persona. My mom's encouragement and help is undeniable; she is the warm guiding light accompanying me along many tenacious roads. I have to be grateful to my aunt Ann, who allowed us to come to the US from Russia.

Very importantly, my brother BoBka and my amazing sister-in-law Dina and their wonderful family deserve a thank-you on so many levels. My niece Gabbi and nephew Daniel literally participated in this thesis by working on the cover page, but they also continuously remind me to take life with a smile.

And lastly, I have to thank my friends, who always make me realize how diverse this world is; some of them are really like my family, particularly Ingrid Adelsberger, Chris Calvert, Ben and Mindy Speciale (who I also am grateful for being my paranifms), Lin Chong, Peilin Chen, Mary DiCarlantonio, Tony Cho, Chris Jandoli, Theresa Fraser, Matt Filow, Matty Baker, Paul Zanlser, and others.

At the end of the day, I consider myself very lucky. And I sincerely hope that my work will improve outcomes in patients with chronic kidney disease. For patients who are already on dialysis, I hope these predictive techniques are likely to prevent morbid events from happening. I also hope that the idea of looking at both oscillatory and non-oscillatory trends can guide clinicians for many years to come. 
Curriculum Vitae 


\section{Curriculum Vitae}

Len Usvyat was born September 7, 1974 in St. Petersburg (Leningrad at the time), Russia. Upon finishing high school, he left Russia to move to New York City in June 1991 with his family. He completed Bachelor's Degree from Queens College in Accounting, Economics and Information Systems in 1996. He further holds a Master's Degree and Certificate in Clinical Epidemiology from the University of Pennsylvania, completed in August 2010. He worked as a project leader at KPMG from 1994 to 2000 and as a manager at Chanel from 2000 to 2002, both in information technology. From 2002 to 2012, he worked as a clinical analysis lead at Renal Research Institute. In 2012, he moved to Fresenius Medical Care's Medical Office in Waltham, MA, USA as a Director of Integrated Care Analytics. 
